\title{
TOXICOLOGICAL PROFILE FOR
} BORON

\author{
U.S. DEPARTMENT OF HEALTH AND HUMAN SERVICES \\ Public Health Service \\ Agency for Toxic Substances and Disease Registry
}

November 2010 


\section{DISCLAIMER}

The use of company or product name(s) is for identification only and does not imply endorsement by the Agency for Toxic Substances and Disease Registry. 


\section{UPDATE STATEMENT}

A Toxicological Profile for Boron Draft for Public Comment was released in October 2007. This edition supersedes any previously released draft or final profile.

Toxicological profiles are revised and republished as necessary. For information regarding the update status of previously released profiles, contact ATSDR at:

Agency for Toxic Substances and Disease Registry

Division of Toxicology and Environmental Medicine/Applied Toxicology Branch

1600 Clifton Road NE

Mailstop F-62

Atlanta, Georgia 30333 
This page is intentionally blank. 


\section{FOREWORD}

This toxicological profile is prepared in accordance with guidelines* developed by the Agency for Toxic Substances and Disease Registry (ATSDR) and the Environmental Protection Agency (EPA). The original guidelines were published in the Federal Register on April 17, 1987. Each profile will be revised and republished as necessary.

The ATSDR toxicological profile succinctly characterizes the toxicologic and adverse health effects information for these toxic substances described therein. Each peer-reviewed profile identifies and reviews the key literature that describes a substance's toxicologic properties. Other pertinent literature is also presented, but is described in less detail than the key studies. The profile is not intended to be an exhaustive document; however, more comprehensive sources of specialty information are referenced.

The focus of the profiles is on health and toxicologic information; therefore, each toxicological profile begins with a public health statement that describes, in nontechnical language, a substance's relevant toxicological properties. Following the public health statement is information concerning levels of significant human exposure and, where known, significant health effects. The adequacy of information to determine a substance's health effects is described in a health effects summary. Data needs that are of significance to protection of public health are identified by ATSDR.

Each profile includes the following:

(A) The examination, summary, and interpretation of available toxicologic information and epidemiologic evaluations on a toxic substance to ascertain the levels of significant human exposure for the substance and the associated acute, subacute, and chronic health effects;

(B) A determination of whether adequate information on the health effects of each substance is available or in the process of development to determine levels of exposure that present a significant risk to human health of acute, subacute, and chronic health effects; and

(C) Where appropriate, identification of toxicologic testing needed to identify the types or levels of exposure that may present significant risk of adverse health effects in humans.

The principal audiences for the toxicological profiles are health professionals at the Federal, State, and local levels; interested private sector organizations and groups; and members of the public.

This profile reflects ATSDR's assessment of all relevant toxicologic testing and information that has been peer-reviewed. Staffs of the Centers for Disease Control and Prevention and other Federal scientists have also reviewed the profile. In addition, this profile has been peer-reviewed by a nongovernmental panel and was made available for public review. Final responsibility for the contents and views expressed in this toxicological profile resides with ATSDR.

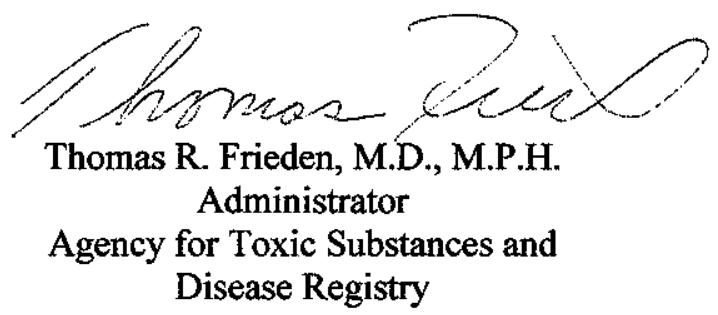


The toxicological profiles are developed under the Comprehensive Environmental Response, Compensation, and Liability Act of 1980, as amended (CERCLA or Superfund). CERCLA section 104(i)(1) directs the Administrator of ATSDR to "...effectuate and implement the health related authorities" of the statute. This includes the preparation of toxicological profiles for hazardous substances most commonly found at facilities on the CERCLA National Priorities List and that pose the most significant potential threat to human health, as determined by ATSDR and the EPA. Section 104(i)(3) of CERCLA, as amended, directs the Administrator of ATSDR to prepare a toxicological profile for each substance on the list. In addition, ATSDR has the authority to prepare toxicological profiles for substances not found at sites on the National Priorities List, in an effort to "...establish and maintain inventory of literature, research, and studies on the health effects of toxic substances" under CERCLA Section 104(i)(1)(B), to respond to requests for consultation under section 104(i)(4), and as otherwise necessary to support the site-specific response actions conducted by ATSDR. 


\section{QUICK REFERENCE FOR HEALTH CARE PROVIDERS}

Toxicological Profiles are a unique compilation of toxicological information on a given hazardous substance. Each profile reflects a comprehensive and extensive evaluation, summary, and interpretation of available toxicologic and epidemiologic information on a substance. Health care providers treating patients potentially exposed to hazardous substances will find the following information helpful for fast answers to often-asked questions.

\section{Primary Chapters/Sections of Interest}

Chapter 1: Public Health Statement: The Public Health Statement can be a useful tool for educating patients about possible exposure to a hazardous substance. It explains a substance's relevant toxicologic properties in a nontechnical, question-and-answer format, and it includes a review of the general health effects observed following exposure.

Chapter 2: Relevance to Public Health: The Relevance to Public Health Section evaluates, interprets, and assesses the significance of toxicity data to human health.

Chapter 3: Health Effects: Specific health effects of a given hazardous compound are reported by type of health effect (death, systemic, immunologic, reproductive), by route of exposure, and by length of exposure (acute, intermediate, and chronic). In addition, both human and animal studies are reported in this section.

NOTE: Not all health effects reported in this section are necessarily observed in the clinical setting. Please refer to the Public Health Statement to identify general health effects observed following exposure.

Pediatrics: Four new sections have been added to each Toxicological Profile to address child health issues:
Section 1.6
How Can (Chemical X) Affect Children?
Section 1.7
Section 3.7
How Can Families Reduce the Risk of Exposure to (Chemical X)?
Section 6.6
Children's Susceptibility
Exposures of Children

Other Sections of Interest:

Section 3.8 Biomarkers of Exposure and Effect

Section 3.11 Methods for Reducing Toxic Effects

\section{ATSDR Information Center \\ Phone: 1-800-CDC-INFO (800-232-4636) or 1-888-232-6348 (TTY) Fax: (770) 488-4178 \\ E-mail: cdcinfo@cdc.gov Internet: http://www.atsdr.cdc.gov}

The following additional material can be ordered through the ATSDR Information Center:

Case Studies in Environmental Medicine: Taking an Exposure History-The importance of taking an exposure history and how to conduct one are described, and an example of a thorough exposure history is provided. Other case studies of interest include Reproductive and Developmental Hazards; Skin Lesions and Environmental Exposures; Cholinesterase-Inhibiting Pesticide Toxicity; and numerous chemical-specific case studies. 
Managing Hazardous Materials Incidents is a three-volume set of recommendations for on-scene (prehospital) and hospital medical management of patients exposed during a hazardous materials incident. Volumes I and II are planning guides to assist first responders and hospital emergency department personnel in planning for incidents that involve hazardous materials. Volume IIIMedical Management Guidelines for Acute Chemical Exposures-is a guide for health care professionals treating patients exposed to hazardous materials.

Fact Sheets (ToxFAQs) provide answers to frequently asked questions about toxic substances.

\section{Other Agencies and Organizations}

The National Center for Environmental Health (NCEH) focuses on preventing or controlling disease, injury, and disability related to the interactions between people and their environment outside the workplace. Contact: NCEH, Mailstop F-29, 4770 Buford Highway, NE, Atlanta, GA 30341-3724 • Phone: 770-488-7000 • FAX: 770-488-7015.

The National Institute for Occupational Safety and Health (NIOSH) conducts research on occupational diseases and injuries, responds to requests for assistance by investigating problems of health and safety in the workplace, recommends standards to the Occupational Safety and Health Administration (OSHA) and the Mine Safety and Health Administration (MSHA), and trains professionals in occupational safety and health. Contact: NIOSH, 200 Independence Avenue, SW, Washington, DC 20201 • Phone: 800-356-4674 or NIOSH Technical Information Branch, Robert A. Taft Laboratory, Mailstop C-19, 4676 Columbia Parkway, Cincinnati, OH 45226-1998 - Phone: 800-35-NIOSH.

The National Institute of Environmental Health Sciences (NIEHS) is the principal federal agency for biomedical research on the effects of chemical, physical, and biologic environmental agents on human health and well-being. Contact: NIEHS, PO Box 12233, 104 T.W. Alexander Drive, Research Triangle Park, NC 27709 • Phone: 919-541-3212.

\section{Referrals}

The Association of Occupational and Environmental Clinics (AOEC) has developed a network of clinics in the United States to provide expertise in occupational and environmental issues. Contact: AOEC, 1010 Vermont Avenue, NW, \#513, Washington, DC 20005 • Phone: 202-347-4976 -FAX: 202-347-4950 • e-mail: AOEC@AOEC.ORG•Web Page: http://www.aoec.org/.

The American College of Occupational and Environmental Medicine (ACOEM) is an association of physicians and other health care providers specializing in the field of occupational and environmental medicine. Contact: ACOEM, 25 Northwest Point Boulevard, Suite 700, Elk Grove Village, IL 60007-1030 • Phone: 847-818-1800 • FAX: 847-818-9266. 


\section{CONTRIBUTORS}

\section{CHEMICAL MANAGER(S)/AUTHOR(S):}

Malcolm Williams, DVM, Ph.D.

Moiz Mumtaz, Ph.D.

Mike Fay, Ph.D.

Franco Scinicariello, M.D.

Kim Jenkins

ATSDR, Division of Toxicology and Environmental Medicine, Atlanta, GA

Mike Lumpkin, Ph.D.

Lara Chappell, Ph.D.

Peter R. McClure, Ph.D., DABT

SRC, Inc., North Syracuse, NY

\section{THE PROFILE HAS UNDERGONE THE FOLLOWING ATSDR INTERNAL REVIEWS:}

1. Health Effects Review. The Health Effects Review Committee examines the health effects chapter of each profile for consistency and accuracy in interpreting health effects and classifying end points.

2. Minimal Risk Level Review. The Minimal Risk Level Workgroup considers issues relevant to substance-specific Minimal Risk Levels (MRLs), reviews the health effects database of each profile, and makes recommendations for derivation of MRLs.

3. Data Needs Review. The Applied Toxicology Branch reviews data needs sections to assure consistency across profiles and adherence to instructions in the Guidance.

4. Green Border Review. Green Border review assures the consistency with ATSDR policy. 
This page is intentionally blank. 


\section{PEER REVIEW}

A peer review panel was assembled for boron. The panel consisted of the following members:

Draft for Public Comment:

1. Michael Dourson, Ph.D., DABT, Toxicological Excellence for Risk Assessment, Cincinnati, Ohio;

2. Curtis Eckhert, Ph.D., University of California Los Angeles, Los Angeles, California; and

3. Joseph Landolph, Ph.D., University of Southern California, Los Angeles, California.

These experts collectively have knowledge of boron's physical and chemical properties, toxicokinetics, key health end points, mechanisms of action, human and animal exposure, and quantification of risk to humans. All reviewers were selected in conformity with the conditions for peer review specified in Section 104(I)(13) of the Comprehensive Environmental Response, Compensation, and Liability Act, as amended.

Scientists from the Agency for Toxic Substances and Disease Registry (ATSDR) have reviewed the peer reviewers' comments and determined which comments will be included in the profile. A listing of the peer reviewers' comments not incorporated in the profile, with a brief explanation of the rationale for their exclusion, exists as part of the administrative record for this compound.

The citation of the peer review panel should not be understood to imply its approval of the profile's final content. The responsibility for the content of this profile lies with the ATSDR. 
This page is intentionally blank. 


\section{CONTENTS}

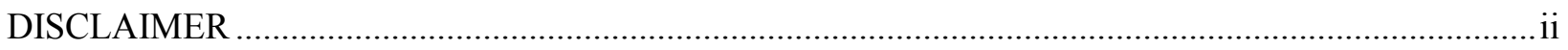

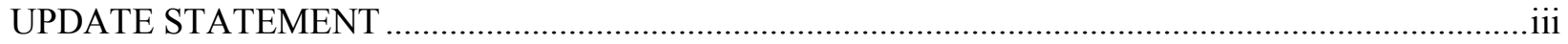

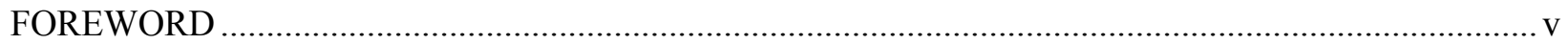

QUICK REFERENCE FOR HEALTH CARE PROVIDERS................................................................ vii

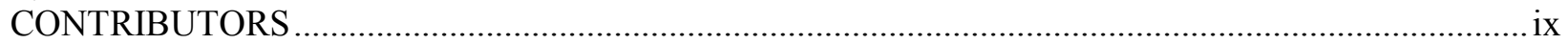

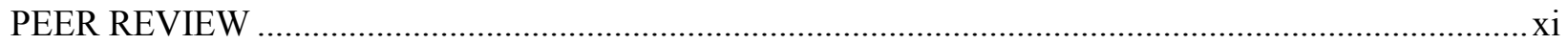

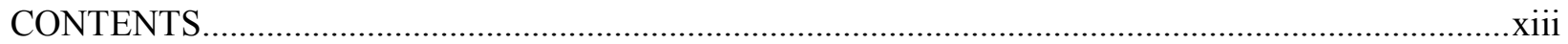

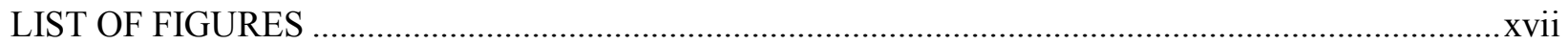

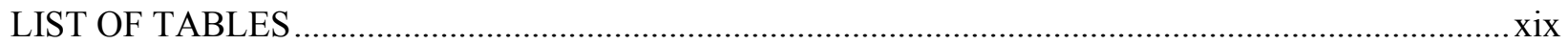

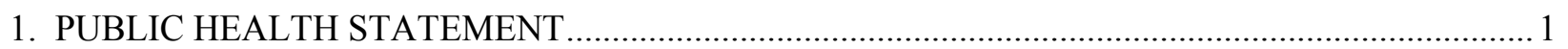

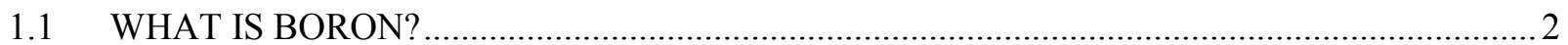

1.2 WHAT HAPPENS TO BORON WHEN IT ENTERS THE ENVIRONMENT?

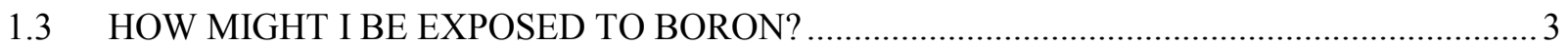

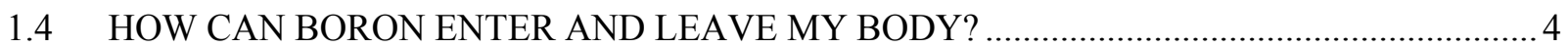

1.5 HOW CAN BORON AFFECT MY HEALTH? ............................................................ 4

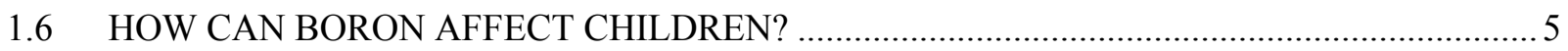

1.7 HOW CAN FAMILIES REDUCE THE RISK OF EXPOSURE TO BORON? ..........................5

1.8 IS THERE A MEDICAL TEST TO DETERMINE WHETHER I HAVE BEEN EXPOSED

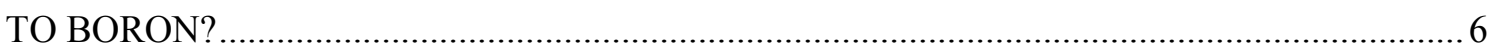

1.9 WHAT RECOMMENDATIONS HAS THE FEDERAL GOVERNMENT MADE TO PROTECT HUMAN HEALTH? ...................................................................................6

1.10 WHERE CAN I GET MORE INFORMATION? …………………………………………. 7

2. RELEVANCE TO PUBLIC HEALTH .................................................................................. 9

2.1 BACKGROUND AND ENVIRONMENTAL EXPOSURES TO BORON IN THE UNITED

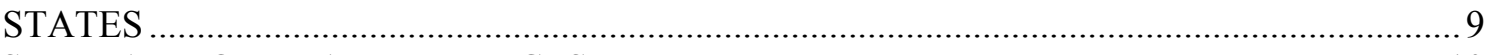

2.2 SUMMARY OF HEALTH EFFECTS .......................................................................... 10

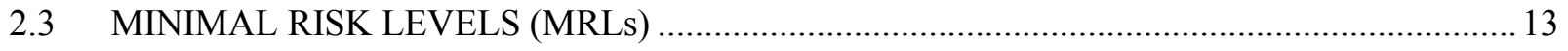

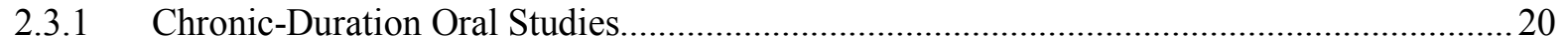

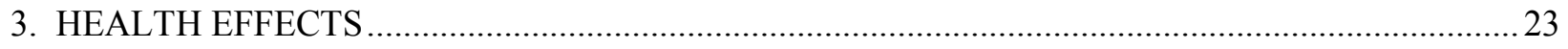

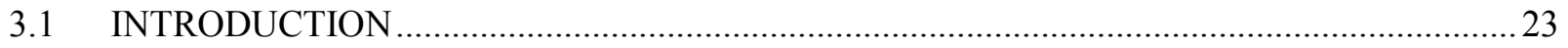

3.2 DISCUSSION OF HEALTH EFFECTS BY ROUTE OF EXPOSURE …………………….....2

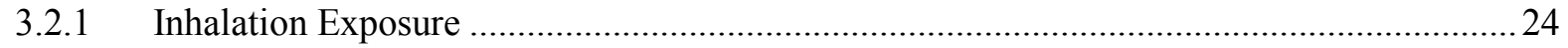

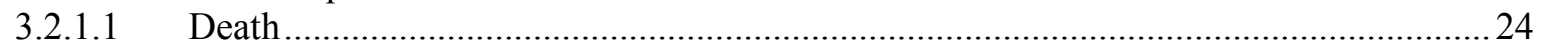

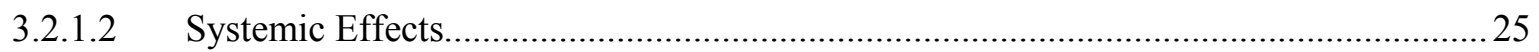

3.2.1.3 Immunological and Lymphoreticular Effects ............................................................38

3.2.1.4 Neurological Effects ............................................................................................. 38

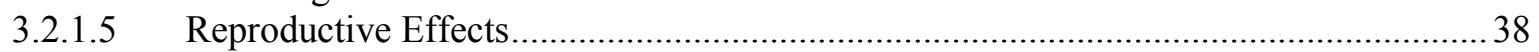

3.2.1.6 Developmental Effects........................................................................................ 38

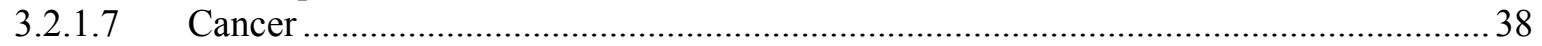

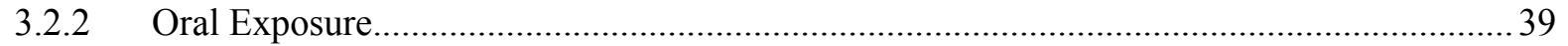

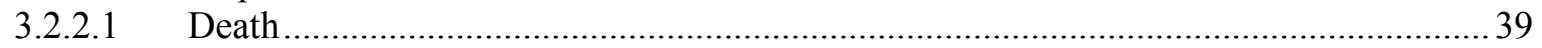

3.2.2.2 Systemic Effects.......................................................................................... 41

3.2.2.3 Immunological and Lymphoreticular Effects ………………………………………... 76

3.2.2.4 Neurological Effects .......................................................................................... 76

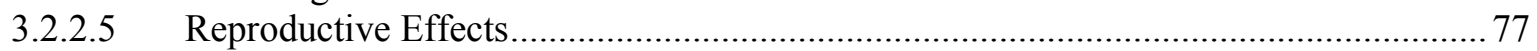

3.2.2.6 Developmental Effects................................................................................... 82 


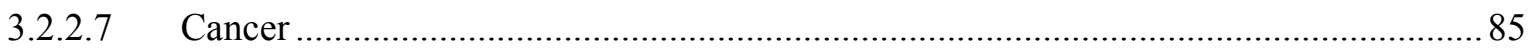

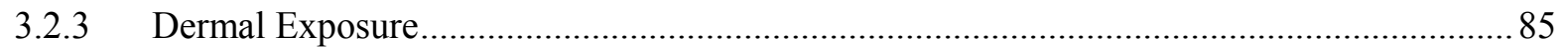

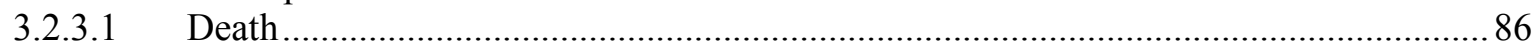

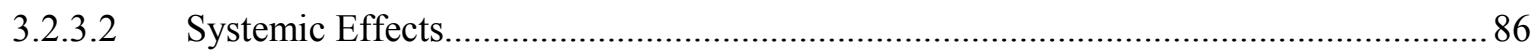

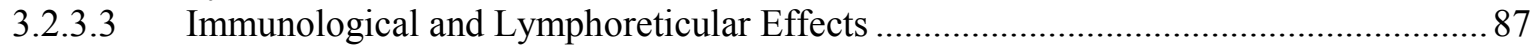

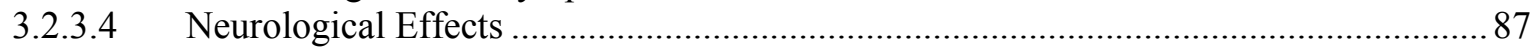

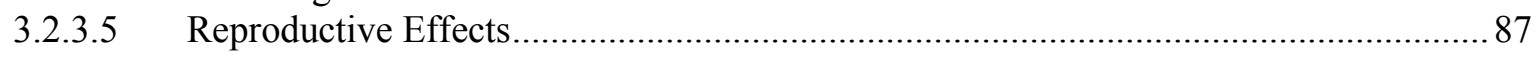

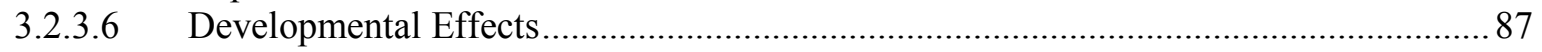

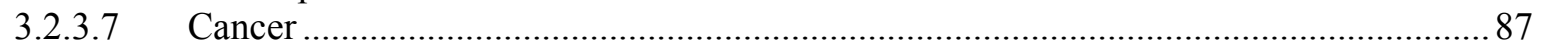

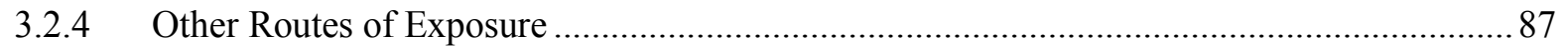

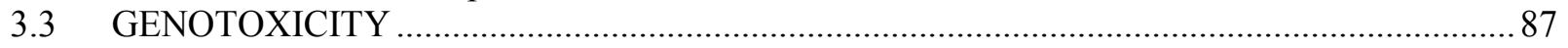

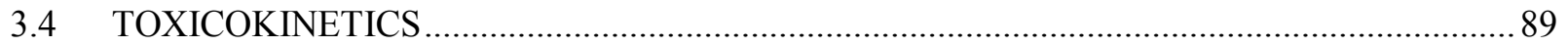

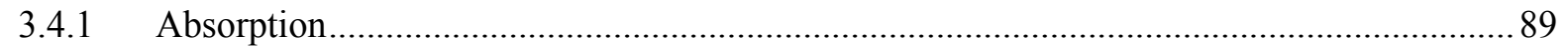

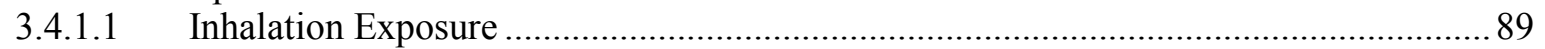

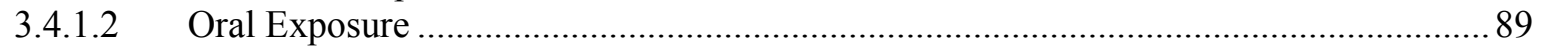

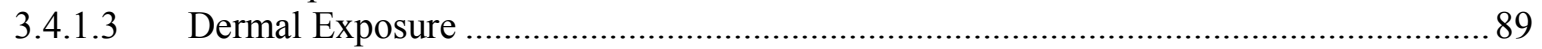

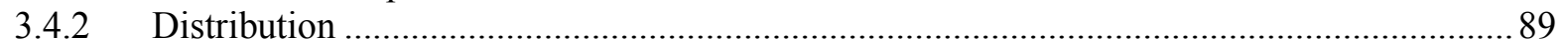

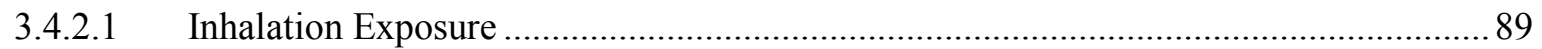

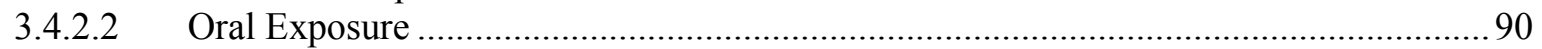

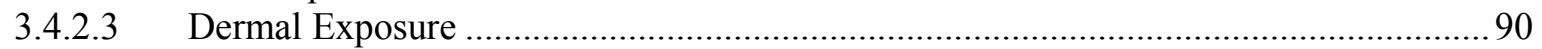

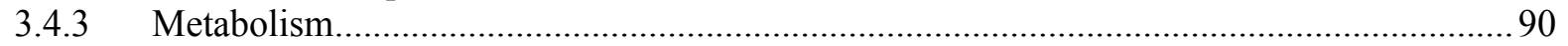

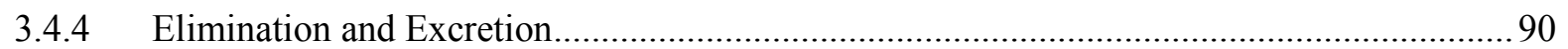

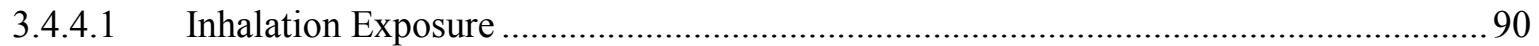

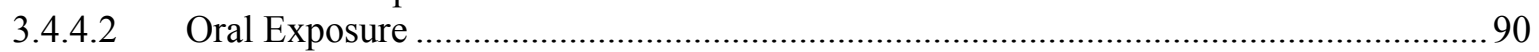

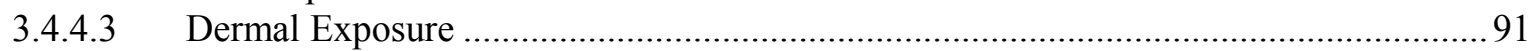

3.4.4.4 Other Routes of Exposure .................................................................................. 91

3.4.5 Physiologically Based Pharmacokinetic (PBPK)/Pharmacodynamic (PD) Models ............. 92

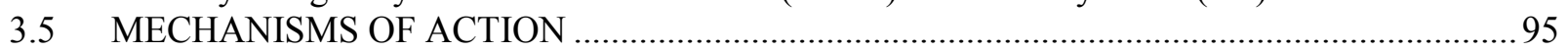

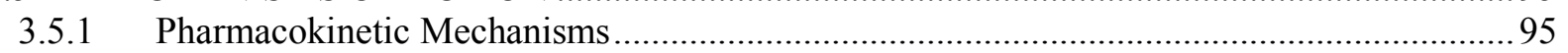

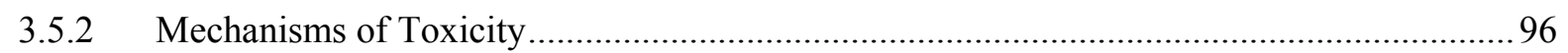

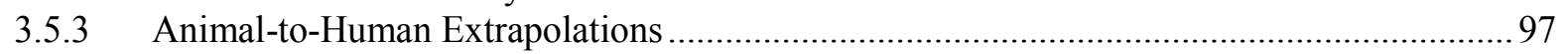

3.6 TOXICITIES MEDIATED THROUGH THE NEUROENDOCRINE AXIS ..............................97

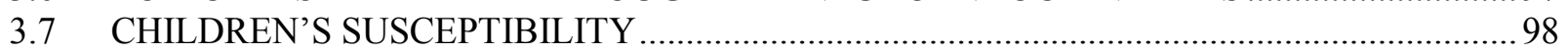

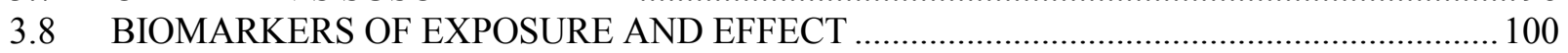

3.8.1 Biomarkers Used to Identify or Quantify Exposure to Boron............................................. 101

3.8.2 Biomarkers Used to Characterize Effects Caused by Boron............................................. 102

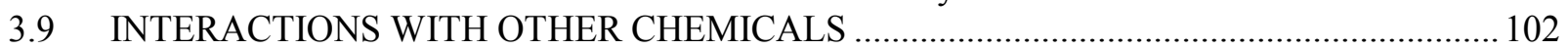

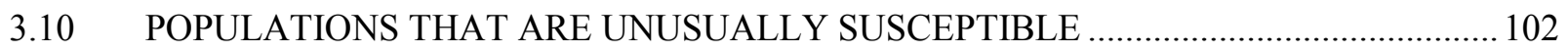

3.11 METHODS FOR REDUCING TOXIC EFFECTS .......................................................... 103

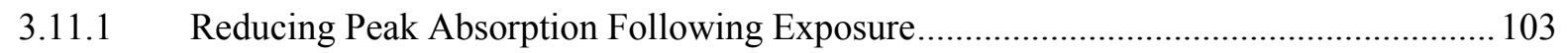

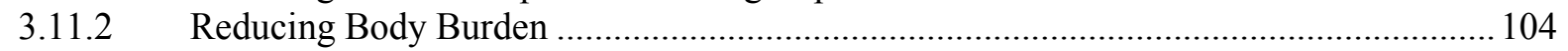

3.11.3 Interfering with the Mechanism of Action for Toxic Effects ....................................... 104

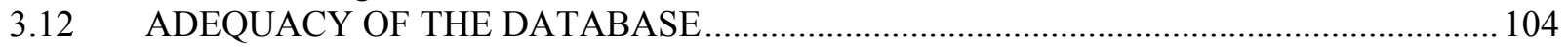

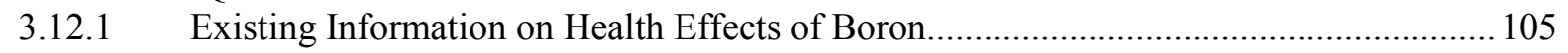

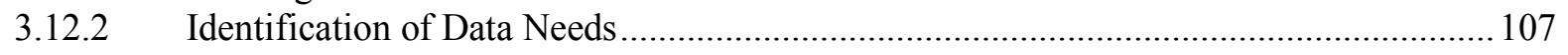

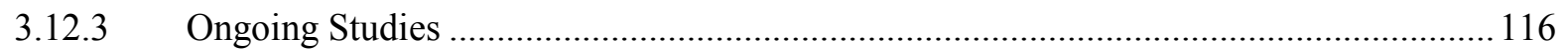

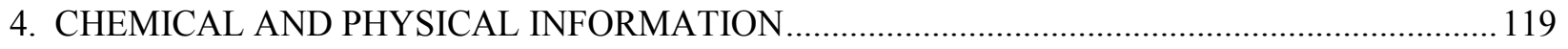

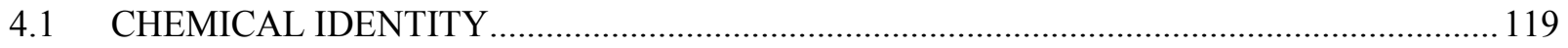

4.2 PHYSICAL AND CHEMICAL PROPERTIES ............................................................. 119 


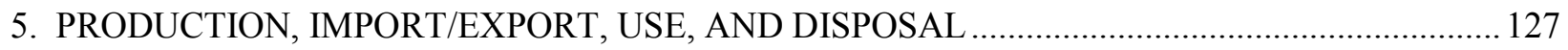

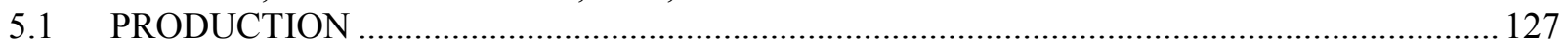

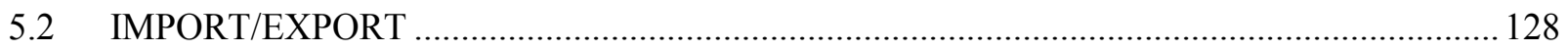

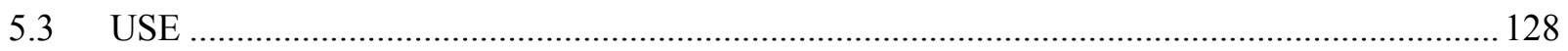

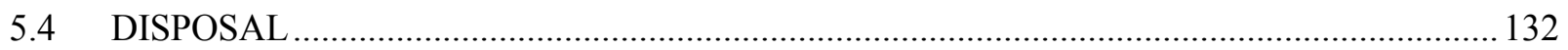

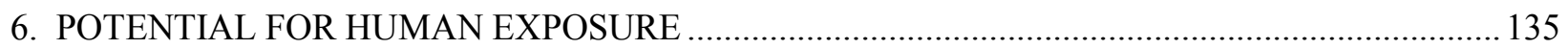

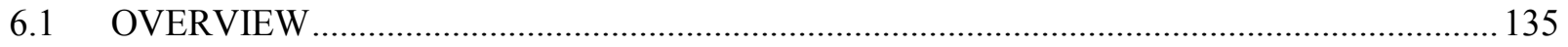

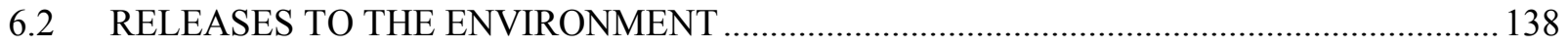

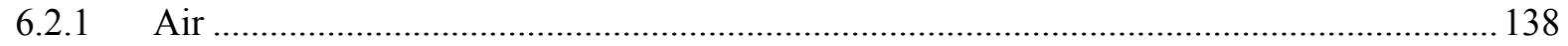

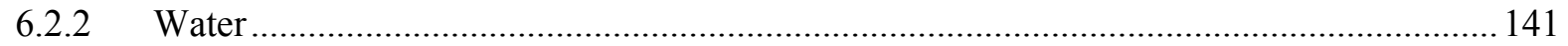

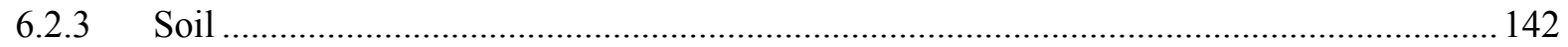

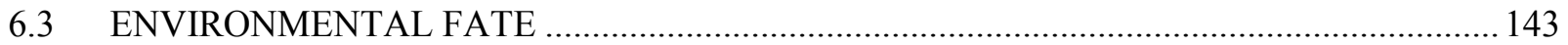

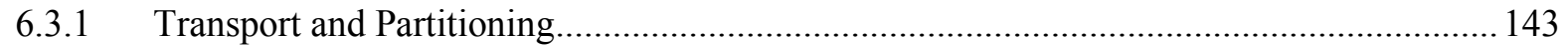

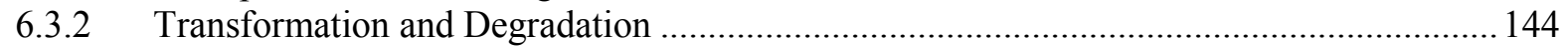

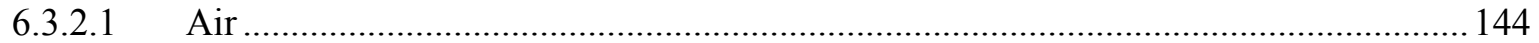

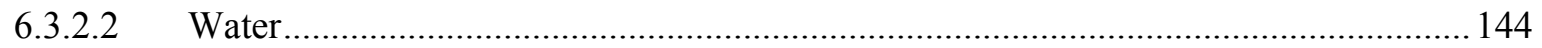

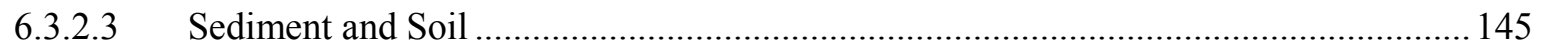

6.4 LEVELS MONITORED OR ESTIMATED IN THE ENVIRONMENT …............................ 145

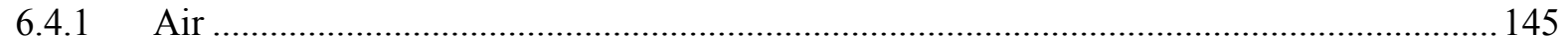

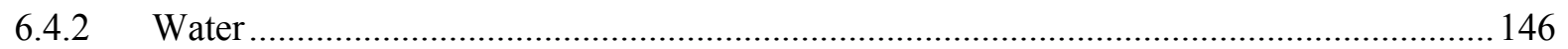

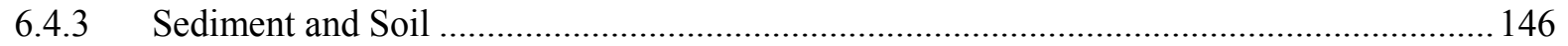

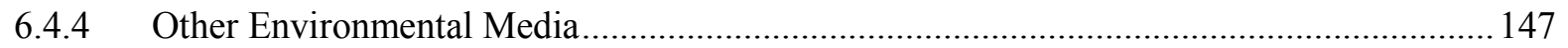

6.5 GENERAL POPULATION AND OCCUPATIONAL EXPOSURE ...................................... 150

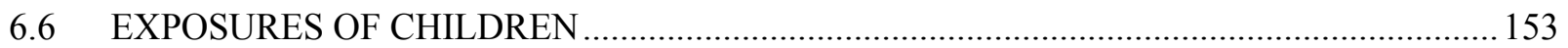

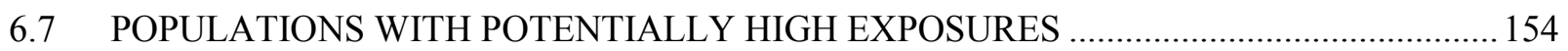

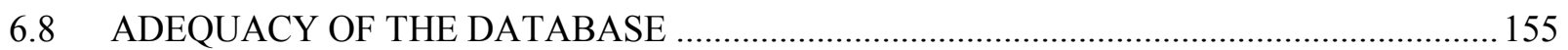

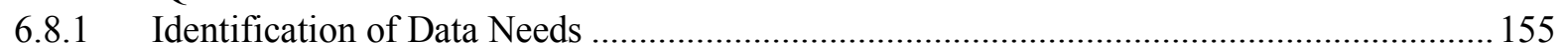

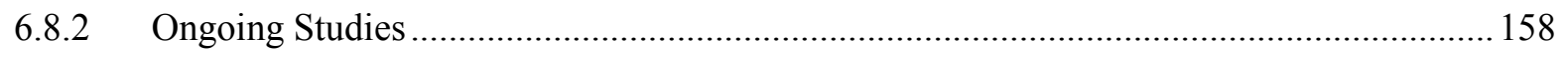

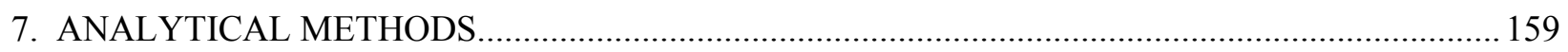

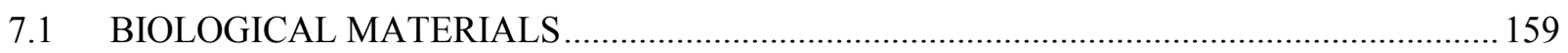

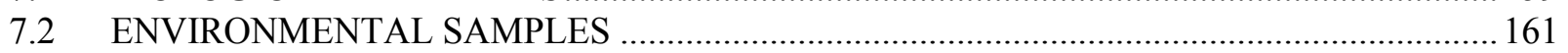

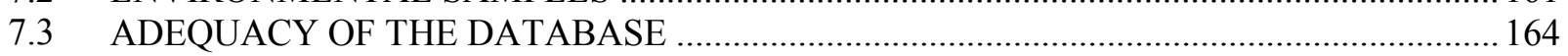

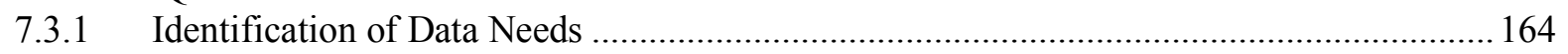

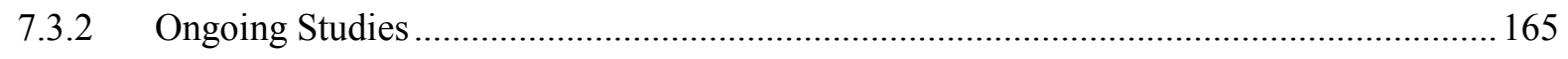

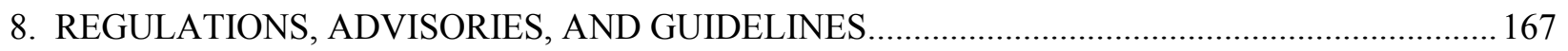

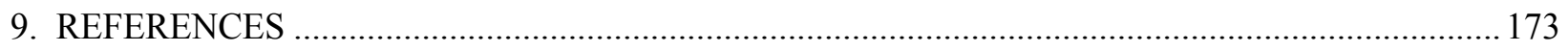

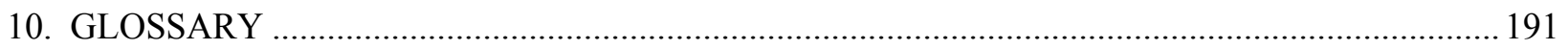

\section{APPENDICES}

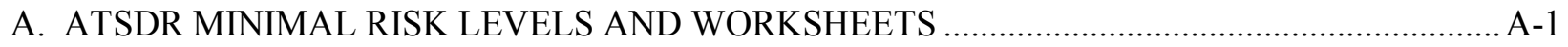

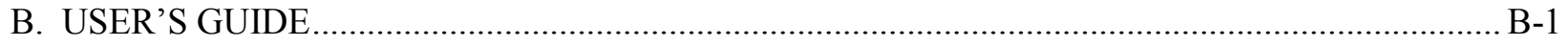

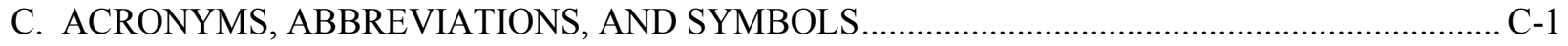

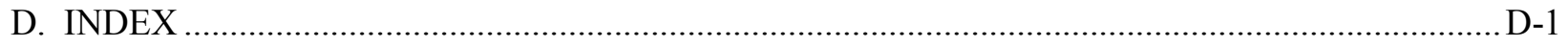


This page is intentionally blank. 


\section{LIST OF FIGURES}

3-1. Levels of Significant Exposure to Boron and Compounds - Inhalation ........................................... 31

3-2. Levels of Significant Exposure to Boron and Compounds - Oral ................................................. 66

3-3. Conceptual Representation of a Physiologically Based Pharmacokinetic (PBPK) Model for a Hypothetical Chemical Substance.

3-4. Existing Information on Health Effects of Boron. 106

6-1. Frequency of NPL Sites with Boron and Selected Boron Contamination. 136 
This page is intentionally blank. 


\section{LIST OF TABLES}

3-1. Levels of Significant Exposure to Boron and Compounds - Inhalation ......................................... 26

3-2. Levels of Significant Exposure to Boron and Compounds - Oral .................................................. 42

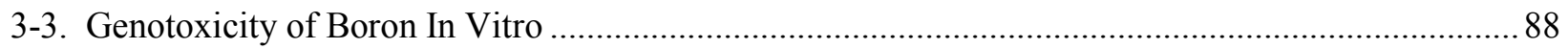

4-1. Chemical Identity of Boron and Selected Boron Compounds ..................................................... 120

4-2. Physical and Chemical Properties of Boron and Selected Boron Compounds............................... 123

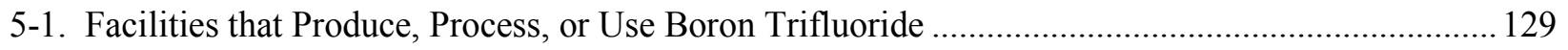

5-2. Facilities that Produce, Process, or Use Boron Trichloride ........................................................ 130

5-3. Current U.S. Manufacturers of Boron and Selected Boron Compounds ......................................... 131

6-1. Releases to the Environment from Facilities that Produce, Process, or Use Boron Trifluoride....... 139

6-2. Releases to the Environment from Facilities that Produce, Process, or Use Boron Trichloride ...... 140

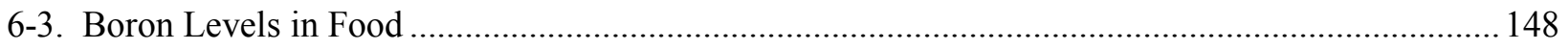

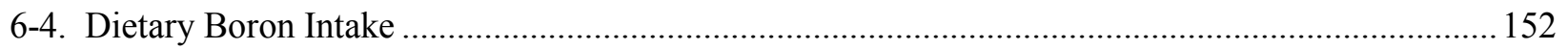

7-1. Analytical Methods for Determining Boron in Biological Materials ........................................... 160

7-2. Analytical Methods for Determining Boron in Environmental Samples...................................... 162

8-1. Regulations, Advisories and Guidelines Applicable to Boron and Boron Compounds ................... 169 
This page is intentionally blank. 


\section{PUBLIC HEALTH STATEMENT}

This public health statement tells you about boron and the effects of exposure to it.

The Environmental Protection Agency (EPA) identifies the most serious hazardous waste sites in the nation. These sites are then placed on the National Priorities List (NPL) and are targeted for long-term federal clean-up activities. Boron and boron compounds have been found in at least 164 of the 1,689 current or former NPL sites, respectively. Although the total number of NPL sites evaluated for this substance is not known, the possibility exists that the number of sites at which boron is found may increase in the future as more sites are evaluated. This information is important because these sites may be sources of exposure and exposure to this substance may be harmful.

When a substance is released either from a large area, such as an industrial plant, or from a container, such as a drum or bottle, it enters the environment. Such a release does not always lead to exposure. You can be exposed to a substance only when you come in contact with it. You may be exposed by breathing, eating, or drinking the substance, or by skin contact.

If you are exposed to boron, many factors will determine whether you will be harmed. These factors include the dose (how much), the duration (how long), and how you come in contact with it. You must also consider any other chemicals you are exposed to and your age, sex, diet, family traits, lifestyle, and state of health. 


\subsection{WHAT IS BORON?}

\begin{tabular}{|c|c|}
\hline $\begin{array}{l}\text { Naturally } \\
\text { occurring }\end{array}$ & $\begin{array}{l}\text { Boron is a widely occurring element in minerals found in the earth's crust. It } \\
\text { is the } 51^{\text {st }} \text { most common element found in the earth's crust and is found at } \\
\text { an average concentration of } 8 \mathrm{mg} / \mathrm{kg} \text { (approximately } 0.0008 \% \text { ). }\end{array}$ \\
\hline $\begin{array}{l}\text { Combines with } \\
\text { oxygen to form } \\
\text { borates }\end{array}$ & $\begin{array}{l}\text { Boron is found in the environment primarily combined with oxygen in } \\
\text { compounds called borates. Common borate compounds include: } \\
\text { - boric acid } \\
\text { - sodium tetraborates (also referred to as borax) } \\
\text { - boron oxide }\end{array}$ \\
\hline $\begin{array}{l}\text { Used to } \\
\text { manufacture } \\
\text { industrial and } \\
\text { consumer } \\
\text { products }\end{array}$ & $\begin{array}{l}\text { Borate-containing minerals are mined and processed to produce borates for } \\
\text { several industrial uses in the United States including: } \\
\text { - glass and ceramics } \\
\text { - } \text { soaps, bleaches, and detergents } \\
\text { - fire retardants } \\
\text { - pesticides }\end{array}$ \\
\hline
\end{tabular}

More information on the properties and uses of boron and boron compounds and how they behave in the environment may be found in Chapters 4, 5, and 6.

\subsection{WHAT HAPPENS TO BORON WHEN IT ENTERS THE ENVIRONMENT?}

\begin{tabular}{|l|l|}
\hline $\begin{array}{l}\text { Released into } \\
\text { air, water, and } \\
\text { soil }\end{array}$ & $\begin{array}{l}\text { Boron can be released into air, water, or soil after natural weathering of soils } \\
\text { and rocks. } \\
\text { Smaller amounts of boron can be released from: }\end{array}$ \\
$\begin{array}{l}-\quad \text { glass manufacturing plants } \\
-\quad \text { coal-burning power plants } \\
\text { copper smelters }\end{array}$ \\
\hline $\begin{array}{l}\text { Is not broken } \\
\text { down }\end{array}$ & $\begin{array}{l}\text { Boricultural fertilizer and pesticide usage } \\
\text { become attached or separated from particles in soil, sediment, and water. }\end{array}$ \\
\hline
\end{tabular}

For more information on boron in the environment, see Chapter 6. 


\subsection{HOW MIGHT I BE EXPOSED TO BORON?}

\begin{tabular}{|c|c|}
\hline Food & $\begin{array}{l}\text { You can be exposed to boron in food, mainly vegetables and fruits, as boron } \\
\text { is an essential element in plants. The average daily intake of boron for } \\
\text { adults is } 1 \text { milligram. }\end{array}$ \\
\hline Water & $\begin{array}{l}\text { Boron is widely distributed in surface water and groundwater. } \\
\text { - the average surface water concentration is about } 0.1 \mathrm{mg} \text { per liter } \\
\text { ( } \mathrm{mg} / \mathrm{L}) \\
\text { boron concentrations in ground water can be as high as } 300 \mathrm{mg} / \mathrm{L} \text { in } \\
\text { areas with natural boron-rich deposits } \\
\text { concentrations up to } 0.4 \mathrm{mg} / \mathrm{L} \text { have been found in most drinking } \\
\text { water samples }\end{array}$ \\
\hline Soil & $\begin{array}{l}\text { Average concentrations of } 26 \text { and } 33 \mathrm{mg} \text { per kilogram }(\mathrm{mg} / \mathrm{kg}) \text { have been } \\
\text { reported in soil. }\end{array}$ \\
\hline Air & $\begin{array}{l}\text { The general public is not likely to be exposed to air contaminated with boron. } \\
\text { The average level of boron in air samples is } 0.00005 \mathrm{mg} \text { boron per cubic } \\
\text { meter of air (mg boron } / \mathrm{m}^{3} \text { ). }\end{array}$ \\
\hline Workplace air & $\begin{array}{l}\text { In workplaces that mine and process borates, boron concentrations in dusty } \\
\text { air samples have been reported to range from about } 0.5 \text { to } 3 \mathrm{mg} \text { boron } / \mathrm{m}^{3} \text {. }\end{array}$ \\
\hline $\begin{array}{l}\text { Consumer } \\
\text { products }\end{array}$ & $\begin{array}{l}\text { Boric acid, anhydrous sodium tetraborate, and sodium tetraborate } \\
\text { decahydrate (borax) are found in consumer products such as: } \\
\text { - laundry detergent } \\
\text { - } \text { pesticides } \\
\text { - } \text { facial creams and cleaners } \\
\text { - } \text { plant foods } \\
\text { household cleaners }\end{array}$ \\
\hline
\end{tabular}

Further information on how you might be exposed to boron is given in Chapter 6 . 


\subsection{HOW CAN BORON ENTER AND LEAVE MY BODY?}

\begin{tabular}{|l|l|}
\hline $\begin{array}{l}\text { Most ingested } \\
\text { boron is } \\
\text { absorbed }\end{array}$ & $\begin{array}{l}\text { Boron can enter your body when you eat food (fruit and vegetables), drink } \\
\text { water containing it, when you breathe borate dust in the air, and when } \\
\text { damaged skin comes in contact with it. }\end{array}$ \\
\hline $\begin{array}{l}\text { Typically leaves } \\
\text { your body within } \\
\text { 4 days }\end{array}$ & $\begin{array}{l}\text { Most of the boron leaves the body in urine. } \\
\text { Over half of the boron taken by mouth can be found in urine within } 24 \text { hours } \\
\text { and the other half can be detected in urine for up to } 4 \text { days. }\end{array}$ \\
\hline
\end{tabular}

Further information on how boron enters and leaves the body is given in Chapter 3.

\subsection{HOW CAN BORON AFFECT MY HEALTH?}

This section looks at studies concerning potential health effects in animal and human studies.

One way to learn whether a chemical will harm people is to determine how the body absorbs, uses, and releases the chemical. For some chemicals, animal testing may be necessary. Animal testing may also help identify health effects such as cancer or birth defects. Without laboratory animals, scientists would lose a basic method for getting information needed to make wise decisions that protect public health. Scientists have the responsibility to treat research animals with care and compassion. Scientists must comply with strict animal care guidelines because laws today protect the welfare of research animals.

The effect of boron on human health depends on how much boron is present, how you are exposed to it, and the length of exposure.

\begin{tabular}{|l|l} 
Exposure in air & $\begin{array}{l}\text { People working in dusty workplaces where borates are mined and } \\
\text { processed have reported irritation of the nose, throat, and eyes. The } \\
\text { irritation does not persist for long periods after leaving the dusty area. }\end{array}$
\end{tabular}




\begin{tabular}{|l|l|}
\hline $\begin{array}{l}\text { Exposure by } \\
\text { ingestion }\end{array}$ & $\begin{array}{l}\text { Humans: Exposure to large amounts of boron (about } 30 \mathrm{~g} \text { of boric acid) } \\
\text { over short periods of time can affect the stomach, intestines, liver, kidney, } \\
\text { and brain and can eventually lead to death. }\end{array}$ \\
$\begin{array}{l}\text { Animals: Studies of dogs, rats, and mice indicate that the male reproductive } \\
\text { organs, especially the testes, are affected if large amounts of boron are } \\
\text { ingested for short or long periods of time. The doses that produced these } \\
\text { effects in animals are more than 1,800 times higher than the average daily } \\
\text { intake of boron in food by adults in the U.S. population. }\end{array}$ \\
$\begin{array}{l}\text { No evidence of cancer was found in a study in which mice were given boric } \\
\text { acid in the diet throughout their lifetime. }\end{array}$
\end{tabular}

More information on the health effects of boron in humans and animals can be found in Chapters 2 and 3.

\subsection{HOW CAN BORON AFFECT CHILDREN?}

This section discusses potential health effects in humans from exposures during the period from conception to maturity at 18 years of age.

\begin{tabular}{|l|l|}
\hline $\begin{array}{l}\text { Children are likely } \\
\text { to have similar } \\
\text { effects as adults }\end{array}$ & $\begin{array}{l}\text { It is likely that children would show the same health effects as adults. We } \\
\text { do not know whether children differ in their susceptibility to the effects of } \\
\text { boron. }\end{array}$ \\
\hline Birth defects & $\begin{array}{l}\text { We do not know whether boron causes birth defects in people. Low birth } \\
\text { weights, birth defects, and developmental delays have occurred in newborn } \\
\text { animals whose mothers were orally exposed to high doses of boron (as } \\
\text { boric acid). The doses that produced these effects in pregnant animals are } \\
\text { more than } 800 \text { times higher than the average daily intake of boron in food } \\
\text { by adult women in the U.S. population. }\end{array}$ \\
\hline
\end{tabular}

\subsection{HOW CAN FAMILIES REDUCE THE RISK OF EXPOSURE TO BORON?}

If your doctor finds that you have been exposed to substantial amounts of boron, ask whether your children might also have been exposed. Your doctor might need to ask your state health department to investigate. 
Boron is part of the natural environment and you will have some exposure from foods and drinking water.

\begin{tabular}{|l|l|}
\hline $\begin{array}{l}\text { Limit children's } \\
\text { exposure to } \\
\text { pesticides }\end{array}$ & $\begin{array}{l}\text { Pesticides containing boron compounds should be used according to their } \\
\text { directions and should be kept away from children. }\end{array}$ \\
\hline $\begin{array}{l}\text { Store household } \\
\text { chemicals out of } \\
\text { reach of young } \\
\text { children }\end{array}$ & $\begin{array}{l}\text { Always store household chemicals in their original labeled containers out of } \\
\text { reach of young children to prevent accidental poisonings. Never store } \\
\text { household chemicals in containers children would find attractive to eat or } \\
\text { drink from, such as old soda bottles. }\end{array}$ \\
\hline $\begin{array}{l}\text { Discourage } \\
\text { children from } \\
\text { eating dirt or } \\
\text { putting hands in } \\
\text { their mouth while } \\
\text { playing with dirt }\end{array}$ & $\begin{array}{l}\text { Children living near waste sites containing boron and boron compounds are } \\
\text { likely to be exposed to higher than normal environmental levels of boron } \\
\text { through breathing in boron-containing dust, touching soil, and eating } \\
\text { contaminated soil. }\end{array}$ \\
\hline $\begin{array}{l}\text { Children should be encouraged to wash their hands frequently, especially } \\
\text { before eating. }\end{array}$ \\
\hline
\end{tabular}

\subsection{IS THERE A MEDICAL TEST TO DETERMINE WHETHER I HAVE BEEN EXPOSED TO BORON?}

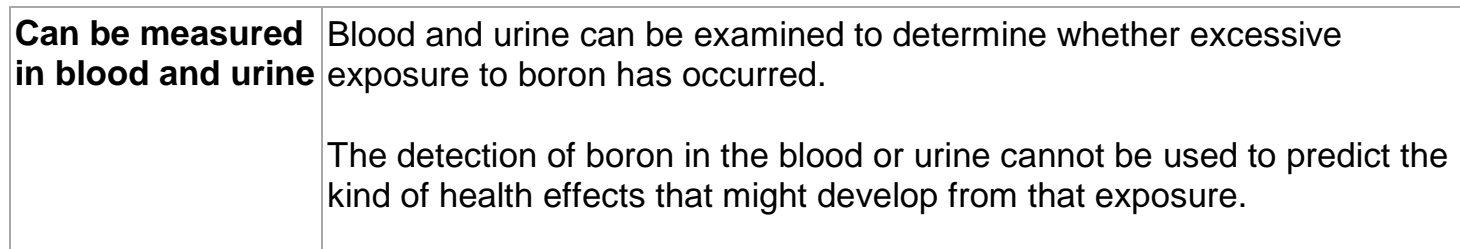

Further information on how boron can be measured in exposed humans is presented in Chapters 3 and 7.

\subsection{WHAT RECOMMENDATIONS HAS THE FEDERAL GOVERNMENT MADE TO PROTECT HUMAN HEALTH?}

The federal government develops regulations and recommendations to protect public health. Regulations can be enforced by law. The EPA, the Occupational Safety and Health Administration (OSHA), and the Food and Drug Administration (FDA) are some federal agencies that develop regulations for toxic substances. Recommendations provide valuable guidelines to protect public health, but cannot be enforced by law. The Agency for Toxic Substances and Disease Registry (ATSDR) and the National Institute for Occupational Safety and Health (NIOSH) are two federal organizations that develop recommendations for toxic substances. 
Regulations and recommendations can be expressed as "not-to-exceed" levels, that is, levels of a toxic substance in air, water, soil, or food that do not exceed a critical value that is usually based on levels that affect animals; they are then adjusted to levels that will help protect humans. Sometimes these not-toexceed levels differ among federal organizations because they used different exposure times (an 8-hour workday or a 24-hour day), different animal studies, or other factors.

Recommendations and regulations are also updated periodically as more information becomes available. For the most current information, check with the federal agency or organization that provides it.

Some regulations and recommendations for boron include the following:

\begin{tabular}{|l|l|}
\hline $\begin{array}{l}\text { Levels in drinking } \\
\text { water set by EPA }\end{array}$ & $\begin{array}{l}\text { The EPA has determined that exposure to boron in drinking water at } \\
\text { concentrations of } 4 \mathrm{mg} / \mathrm{L} \text { for one day or } 0.9 \mathrm{mg} / \mathrm{L} \text { for } 10 \text { days is not } \\
\text { expected to cause any adverse effects in a child. }\end{array}$ \\
$\begin{array}{l}\text { The EPA has determined that lifetime exposure to } 1 \mathrm{mg} / \mathrm{L} \text { boron is not } \\
\text { expected to cause any adverse effects. }\end{array}$ \\
\hline $\begin{array}{l}\text { Levels in } \\
\text { workplace air set } \\
\text { by OSHA }\end{array}$ & $\begin{array}{l}\text { OSHA set a legal limit of } 15 \mathrm{mg} / \mathrm{m}^{3} \text { for boron oxide in air averaged over an } \\
\text { 8-hour work day. }\end{array}$ \\
\hline
\end{tabular}

Additional information on governmental regulations regarding boron can be found in Chapter 8 .

\subsection{WHERE CAN I GET MORE INFORMATION?}

If you have any more questions or concerns, please contact your community or state health or environmental quality department, or contact ATSDR at the address and phone number below.

ATSDR can also tell you the location of occupational and environmental health clinics. These clinics specialize in recognizing, evaluating, and treating illnesses that result from exposure to hazardous substances. 
Toxicological profiles are also available on-line at www.atsdr.cdc.gov and on CD-ROM. You may request a copy of the ATSDR ToxProfiles ${ }^{\mathrm{TM}}$ CD-ROM by calling the toll-free information and technical assistance number at 1-800-CDCINFO (1-800-232-4636), by e-mail at cdcinfo@cdc.gov, or by writing to:

\author{
Agency for Toxic Substances and Disease Registry \\ Division of Toxicology and Environmental Medicine \\ 1600 Clifton Road NE \\ Mailstop F-62 \\ Atlanta, GA 30333 \\ Fax: 1-770-488-4178
}

Organizations for-profit may request copies of final Toxicological Profiles from the following:

National Technical Information Service (NTIS)

5285 Port Royal Road

Springfield, VA 22161

Phone: $1-800-553-6847$ or $1-703-605-6000$

Web site: http://www.ntis.gov/ 


\section{RELEVANCE TO PUBLIC HEALTH}

\subsection{BACKGROUND AND ENVIRONMENTAL EXPOSURES TO BORON IN THE UNITED STATES}

Boron is a widely occurring element in minerals found in the earth's crust. It is the $51^{\text {st }}$ most common element found in the earth's crust and is found at an average concentration of $8 \mathrm{mg} / \mathrm{kg}$ (approximately $0.0008 \%$ ). It is found in the environment primarily combined with oxygen in compounds called borates. Common borate compounds include boric acid, salts of boric acid (e.g., sodium tetraborates, which are also referred to as borax), and boron oxide. U.S. borate mining and production mainly occurs in Kern, San Bernardino, and Inyo Counties, California. Borate-containing minerals are mined and processed to produce borates for several industrial uses in the United States. Industrial uses include glass and ceramics (70\%), soaps, bleaches, and detergents (4\%), fire retardants (2\%), and agriculture (2\%). Other uses, including metallurgy, nuclear applications, sale to distributors, addition to enamels and glazes, and ingredients in cosmetics or medical preparations, make up the remaining 19\%. There are 189 pesticide products registered in the United States that contain boric acid or one of its sodium salts as an active ingredient.

Human exposure to boron, typically as borates or boric acid, may occur through ingestion of food and water, or through use of pesticides containing boron compounds, inhalation of boron-containing powders or dusts, or the use of boron from cosmetics or medical preparations. The most appreciable boron exposure to the general population is likely to be through ingestion of food (as boron is an essential element in plants) and, to a lesser extent, water. Mean daily intakes of boron for male and female adults were reported to be 1.28 and $1.0 \mathrm{mg}$ boron/day. Consumption of fruits and vegetables contribute largely to boron intake in the human diet. Boron levels reported in drinking water generally range from $<1$ to $3 \mathrm{mg}$ boron/L.

Boron concentrations in ambient non-occupational air samples have been reported to range from $<5 \times 10^{-7}$ to $8 \times 10^{-5} \mathrm{mg}$ boron $/ \mathrm{m}^{3}$, with an average concentration of $2 \times 10^{-5} \mathrm{mg}$ boron $/ \mathrm{m}^{3}$. Workers in other industries, including manufacture of fiberglass and other glass products, cleaning and laundry products, fertilizers, pesticides, and cosmetics, may also be exposed to boron compounds. Mean dust concentrations ranging from 3.3 to $18 \mathrm{mg}$ particulates $/ \mathrm{m}^{3}$ were measured in air samples from U.S. facilities where borax was packaged and shipped. Dust samples in these facilities were predominantly composed of various types of borates and ranged from 11.8 to $15.2 \%$ boron by weight. Using the midpoint of this range of boron percentages in the dusts (13.5\% boron), boron concentrations in air from 
these workplaces are estimated to have ranged from 0.45 to $2.43 \mathrm{mg}$ boron $/ \mathrm{m}^{3}$. In another study of dust concentrations in air samples from a U.S. borax production facility, mean total dust concentrations ranged from 0.29 to $18.95 \mathrm{mg}$ particulates $/ \mathrm{m}^{3}$ (approximately $0.02-1.50 \mathrm{mg}$ boron $/ \mathrm{m}^{3}$, using the midpoint (7.9\%) of the ranges of average boron content in these dusts).

The average surface water boron concentration in the United States is about $0.1 \mathrm{mg}$ boron/L, but concentrations vary greatly, depending on boron content of local geologic formations and anthropogenic sources of boron. Several studies have measured boron concentrations in water in those areas of California with boron-rich deposits. Reported high boron concentrations in surface waters ranged from $15 \mathrm{mg}$ boron/L in coastal drainage waters to $360 \mathrm{mg}$ boron/L in a boron-rich lake. Groundwater boron concentrations $>100 \mathrm{mg}$ boron/L are common in California. Average concentrations of 26 and $33 \mathrm{mg}$ boron/kg soil have been reported in soils in the United States, with concentrations ranging up to $300 \mathrm{mg}$ boron/kg. For a more complete discussion of possible exposures to boron, see Chapter 6 of the profile.

\subsection{SUMMARY OF HEALTH EFFECTS}

Most of the inhalation toxicity data for boron involves human or animal exposure to borate dusts such as boric acid, boron oxide, or various hydration states of sodium borate salts (anhydrous, pentahydrate, and decahydrate, also referred to borax) or other borate salts (e.g., calcium borate). Equivalent boron doses have been calculated throughout this assessment by multiplying reported doses by an adjustment factor relating the molar mass ratio of boron in a given compound to the total molar mass. Thus, the dose adjustment factors are 0.175 for boric acid, 0.113 for disodium borate decahydrate, 0.151 for disodium borate pentahydrate, 0.215 for anhydrous borate, and 0.155 for boron oxide.

The primary health effects associated with inhalation exposure of humans to boron are acute respiratory irritation. Sensations of "feel” just below the self-reported threshold for nasal irritation and significant increases in mass of nasal secretions have been observed in well-controlled laboratory studies of exercising volunteers exposed for short durations (up to 47 minutes) to $1.5 \mathrm{mg}$ boron $/ \mathrm{m}^{3}$ as boric acid or sodium borate dusts (Cain et al. 2004, 2008).

Acute-duration exposures of mining and processing workers to $0.44-3.1 \mathrm{mg}$ boron $/ \mathrm{m}^{3}$ (5.7-14.6 $\mathrm{mg}$ particulates $/ \mathrm{m}^{3}$ ) as sodium borate dusts has been associated with mild irritation of the eyes, throat, and nose, as well as cough and breathlessness. No exposure-related changes in lung function were observed in nonsmoking workers; a decrease in 1 second forced expiratory volume $\left(\mathrm{FEV}_{1}\right)$ was observed in 
workers who smoked and were exposed to higher concentrations of boron. However, a re-examination of the workers 7 years later did not result in boron-related alterations in lung function. Animal studies of inhalation exposure to boron are restricted to a series of studies that found no histological changes in a comprehensive examination of tissues (including the respiratory tract) from rats exposed to aerosols of boron oxide (6 hours/day, 5 days/week) at concentrations of $73 \mathrm{mg}$ boron $/ \mathrm{m}^{3}$ for 10 weeks, $27 \mathrm{mg}$ boron $/ \mathrm{m}^{3}$ for 12 weeks, or $12 \mathrm{mg}$ boron $/ \mathrm{m}^{3}$ for 24 weeks. There was some indication of local irritation of the external nares in rats exposed to $73 \mathrm{mg}$ boron $/ \mathrm{m}^{3}$ for 10 weeks. A limited examination of dogs exposed to $9 \mathrm{mg}$ boron $/ \mathrm{m}^{3}$ did not find hematological alterations or evidence of liver damage, evaluated using the sulfobromophthalein retention test.

In contrast, diborane gas $\left(\mathrm{B}_{2} \mathrm{H}_{4}\right)$ is a potent respiratory tract toxicant. Exposure of mice to diborane gas at a concentration of $5 \mathrm{ppm}$ diborane $\left(1.7 \mathrm{mg}\right.$ boron $\left./ \mathrm{m}^{3}\right)$ for 2 weeks produced severe damage to the lungs including pulmonary congestion, bleeding, and edema. Slight changes (infiltration of polymorphous neutrophil in peribronchiolar region) were observed at $0.7 \mathrm{ppm}$ diborane $\left(0.2 \mathrm{mg}\right.$ boron $\left./ \mathrm{m}^{3}\right)$. However, diborane gas is expected to have a very short half-life in the environment and is not expected to be a significant environmental toxicant, except in workplaces where it might be used or manufactured and accidentally released.

Human case reports have shown that boron can be lethal following short-term oral exposure at high doses, although the dose estimation can be quite imprecise and variability in human responses to acute exposure is quite large. The minimal lethal dose of ingested boron (as boric acid) was reported to be $2-3 \mathrm{~g}$ in infants, 5-6 g in children, and 15-20 g in adults. However, a review of 784 human poisonings with boric acid (10-88 g) reported no fatalities, with $88 \%$ of cases being asymptomatic. Liver, kidney, central nervous system, and gastrointestinal effects and skin lesions have been found in lethal cases following ingestion of boron, but death has been attributed to respiratory failure. Surveys of Turkish and Chinese populations with elevated levels of borate salts in drinking water (9-25 mg boron/L) found no associations for chronic-duration exposure with reproductive effects. The essentiality of boron has been established for most plants and some animals, but not in humans. The use of boron as a dietary supplement has not been endorsed by the Food and Nutrition Board/Institute of Medicine and did not result in increased plasma testosterone or strength levels in bodybuilders.

Oral exposure animal studies have clearly identified the reproductive system and developing fetus as the most sensitive targets of boron toxicity. Adverse developmental effects have been identified for acuteand intermediate-duration exposures. Decreases in the number of live fetuses and litters, decreases in 
body weight, and increases in the occurrence of external, visceral, and cardiovascular malformations were observed in the fetuses of rabbits administered $44 \mathrm{mg}$ boron/kg/day on gestation days 6-19; no developmental effects were observed at $22 \mathrm{mg}$ boron/kg/day. Following intermediate-duration exposure, decreases in body weight and increases in the occurrence of skeletal malformations have been observed in the fetuses of rats exposed to $13 \mathrm{mg}$ boron/kg/day on gestation days 0-20; a no-observed-adverse-effect level (NOAEL) of $10 \mathrm{mg}$ boron/kg/day was identified. Reproductive effects have been observed at higher doses. Histological alterations in the testes and sperm effects have been observed in rats administered $88 \mathrm{mg}$ boron/ $\mathrm{kg} /$ day for 2 weeks; the NOAEL was $44-53 \mathrm{mg}$ boron/ $\mathrm{kg} /$ day. Intermediate-duration exposure resulted in histological alterations in the testes and associated effects on spermatogenesis in rats exposed to doses of $\geq 26 \mathrm{mg}$ boron $/ \mathrm{kg} /$ day. No viable sperm were observed in male rats exposed to $101 \mathrm{mg}$ boron/kg/day for 14 weeks. Impaired ovulation and failure to conceive was also observed in female rats (mated with unexposed males) exposed to $116 \mathrm{mg}$ boron $/ \mathrm{kg} /$ day for 14 weeks prior to mating. A NOAEL of $30 \mathrm{mg}$ boron/kg/day was identified for reproductive effects (testicular atrophy) in males in a 3-generation rat study. Testicular atrophy has also been observed in rats exposed to $81 \mathrm{mg}$ boron/kg/day and mice exposed to $201 \mathrm{mg}$ boron/kg/day for 2 years; no testicular alterations were observed at 24 or $79 \mathrm{mg}$ boron/kg/day, respectively.

In addition to the developmental and reproductive effects, several systemic effects have been observed in orally exposed animals. Consistently observed effects following intermediate and chronic exposure include hematological alterations (decreases in hemoglobin levels and splenic hematopoeisis) and desquamated skin on the paw; these effects have been observed at doses of $\geq 60 \mathrm{mg}$ boron $/ \mathrm{kg} / \mathrm{day}$. Chronic inflammation and coagulative necrosis have also been observed in the livers of mice exposed to $79 \mathrm{mg}$ boron/kg/day for 2 years.

The primary health effects associated with dermal exposure are irritation of the eyes and reversible skin changes. Case reports of human occupational exposures have suggested that acute dermal exposure to boron as borax may cause focal alopecia of the scalp. However, as this effect has been reported in only three cases with no estimate of dose and involved co-exposure to high levels of other organic solvents, this association is uncertain. In animals, ocular instillation of $50 \mathrm{mg}$ boron oxide (7.8 $\mathrm{mg}$ boron) dust resulted in conjunctivitis, while instillation of a sodium perborate monohydrate solution containing $6.3 \mathrm{mg}$ boron into the eyes of rabbits caused mild irritancy of the epithelium and superficial stroma.

No epidemiology studies have identified an association between boron exposure and development of cancer. However, some investigators have suggested that boron exposure in drinking water may be 
associated with lower incidences of some types of cancer in humans. Intermediate-duration oral exposure of boric acid to mice that had been implanted with prostate tumor cells resulted in significantly reduced tumor growth and reduced tumor serum antigen levels. Chronic-duration oral studies in rats, mice, and dogs involving dietary exposure to boric acid or borax have not found significant increases in neoplastic lesions. In vitro genotoxicity assays have given predominantly negative results. The International Agency for Research on Cancer (IARC), the National Toxicology Program (NTP), and EPA have not classified boron for human carcinogenicity.

\subsection{MINIMAL RISK LEVELS (MRLS)}

Estimates of exposure levels posing minimal risk to humans (MRLs) have been made for boron. An MRL is defined as an estimate of daily human exposure to a substance that is likely to be without an appreciable risk of adverse effects (noncarcinogenic) over a specified duration of exposure. MRLs are derived when reliable and sufficient data exist to identify the target organ(s) of effect or the most sensitive health effect(s) for a specific duration within a given route of exposure. MRLs are based on noncancerous health effects only and do not consider carcinogenic effects. MRLs can be derived for acute, intermediate, and chronic duration exposures for inhalation and oral routes. Appropriate methodology does not exist to develop MRLs for dermal exposure.

Although methods have been established to derive these levels (Barnes and Dourson 1988; EPA 1990), uncertainties are associated with these techniques. Furthermore, ATSDR acknowledges additional uncertainties inherent in the application of the procedures to derive less than lifetime MRLs. As an example, acute inhalation MRLs may not be protective for health effects that are delayed in development or are acquired following repeated acute insults, such as hypersensitivity reactions, asthma, or chronic bronchitis. As these kinds of health effects data become available and methods to assess levels of significant human exposure improve, these MRLs will be revised.

\section{Inhalation MRLs}

- An MRL of $0.3 \mathrm{mg} / \mathrm{m}^{3}$ has been derived for acute-duration inhalation exposure (14 days or less) to boron.

The available information on the toxicity of inhaled boron comes from an occupational exposure study (Wegman et al. 1994) and two human experimental studies (Cain et al. 2004, 2008). These studies identified increases in nasal secretions as the most sensitive effect. Nasal and throat irritation were 
reported by volunteers exposed to $\geq 4.5 \mathrm{mg}$ boron $/ \mathrm{m}^{3}$ ( $\geq 30 \mathrm{mg}$ sodium borate $/ \mathrm{m}^{3}$ ) for 20 minutes while exercising (Cain et al. 2004). These investigators identified a minimal lowest-observed-adverse-effect level (LOAEL) of $1.5 \mathrm{mg}$ boron $/ \mathrm{m}^{3}$ for increased nasal secretions in exercising volunteers exposed for 47 minutes (Cain et al. 2008). Nose, eye, and throat irritation were self-reported by workers at a borax processing facility exposed to a 6-hour time-weighted average (TWA) concentration ranging from 0.44 to $3.1 \mathrm{mg}$ boron $/ \mathrm{m}^{3}$ (5.7-14.6 mg particulates $/ \mathrm{m}^{3}$ ) (Wegman et al. 1994), although identification of a NOAEL or LOAEL in this study is problematic (see next paragraph). The identification of the respiratory tract as the most sensitive target of toxicity is supported by longer-term animal studies (Wilding et al. 1959) that found no adverse systemic effects in rats or dogs exposed to higher concentrations (9-72 mg boron $\left./ \mathrm{m}^{3}\right)$.

The Cain et al. (2004, 2008) studies were selected over the Wegman et al. (1991, 1994) studies as the basis of the acute-duration inhalation MRL for boron because they identified a NOAEL based on observations from carefully-controlled exposures of volunteers. The Wegman et al. $(1991,1994)$ data are problematic for identifying a NOAEL or LOAEL because the average irritation intensity level reported prior to a work shift was very close to the average intensity level reported for all incidents of nasal irritation. Area dust samples taken in the areas in which workers reported respiratory irritation varied over 4 orders of magnitude, ranging from 0.12 to $205 \mathrm{mg}$ particulate $/ \mathrm{m}^{3}$. The contribution of non-boroncontaining dusts to the reported irritancy cannot be ascertained. Further, the best 0.25 -hour TWA data (confirmed by worker response and simultaneous air sampling by a study technician) suggest that the probability of workers reporting incidences of nasal irritation occurred when estimated boron concentrations reached $0.8-1.2 \mathrm{mg}$ boron $/ \mathrm{m}^{3}$, which is very similar to the laboratory findings of Cain et al. (2004, 2008).

Cain et al. $(2004,2008)$ trained male and female volunteers to recognize the difference in chemesthetic feel (pungency or irritancy) of various levels of $\mathrm{CO}_{2}$ offered to the eyes, nose, and throat. Exposures of $\geq 17.7 \% \mathrm{CO}_{2}$ resulted in a "feel" described by the volunteers as irritating. Twelve male volunteers were exposed to $0,0.8,1.5,3.0,4.5$, or $6.0 \mathrm{mg}$ boron $/ \mathrm{m}^{3}(0,5,10,20,30$, or $40 \mathrm{mg}$ sodium borate pentahydrate $/ \mathrm{m}^{3}$ ) for 20 minutes while performing light exercise (Cain et al. 2004). At 5-minute intervals, they reported the magnitude of "feel" of borate dust in terms of equivalent $\mathrm{CO}_{2}$ irritancy. They were also observed for changes in nasal secretions (by mass) and nasal airway resistance. In a similar fashion, six male and six female volunteers were exposed to $1.5 \mathrm{mg}$ boron $/ \mathrm{m}^{3}$ (10 $\mathrm{mg}$ sodium borate $/ \mathrm{m}^{3}$ ) or 0.4 to $1.8 \mathrm{mg}$ boron $/ \mathrm{m}^{3}$ (2.5 to $10 \mathrm{mg}$ boric acid $/ \mathrm{m}^{3}$ ) for 47 minutes while exercising. They reported the magnitude of "feel" of boric acid and borate dusts in terms of equivalent $\mathrm{CO}_{2}$ irritancy. They were 
also observed for changes in nasal secretions (by mass), nasal airway resistance, and respiration frequency.

Male volunteers exposed to $\leq 3.0 \mathrm{mg}$ boron $/ \mathrm{m}^{3}$ ( $\leq 20 \mathrm{mg}$ sodium borate $/ \mathrm{m}^{3}$ ) for 20 minutes reported increasingly higher magnitude of feel of the dust in eyes, nose, and throat. However, the mean reported level of feel, compared to equivalent $\mathrm{CO}_{2}$ levels, was not considered irritating by the study subjects. The mean reported perception of feel at $\geq 4.5 \mathrm{mg}$ boron $/ \mathrm{m}^{3}\left(\geq 30\right.$ sodium borate $\left./ \mathrm{m}^{3}\right)$ was reported to feel irritating to the nose (Cain et al. 2004). Significantly increased nasal secretions (by mass) occurred at $1.5 \mathrm{mg}$ boron $/ \mathrm{m}^{3}$ (10 $\mathrm{mg}$ sodium borate $/ \mathrm{m}^{3}$ ), but not $0.8 \mathrm{mg}$ boron $/ \mathrm{m}^{3}$ (5 mg sodium borate $/ \mathrm{m}^{3}$ ) (Cain et al. 2004). Similarly, male and female volunteers exposed to $1.5 \mathrm{mg}$ boron $/ \mathrm{m}^{3}$ (10 $\mathrm{mg}$ sodium borate $/ \mathrm{m}^{3}$ ) or $1.8 \mathrm{mg}$ boron $/ \mathrm{m}^{3}$ ( $10 \mathrm{mg}$ boric acid $/ \mathrm{m}^{3}$ ) for 47 minutes while exercising reported a mean sense of feel that initially increased and peaked at the equivalent of slightly less than $17.7 \% \mathrm{CO}_{2}$. These boron exposures, with a mean reported feel equivalent to $<17.7 \% \mathrm{CO}_{2}$, were considered non-irritating by the study subjects, although increases in nasal secretions were observed at $1.8 \mathrm{mg}$ boron $/ \mathrm{mg}^{3}$ (10 mg boric acid $/ \mathrm{m}^{3}$ ), but not at $0.9 \mathrm{mg}$ boron $/ \mathrm{mg}^{3}$ (5 mg boric acid/ $/ \mathrm{m}^{3}$ ) (Cain et al. 2008). Nasal secretions increased above controls for the first 20 minutes (as observed by Cain et al. 2004), but did not accumulate thereafter. Nasal airway resistance decreased, then increased as a result of exercise, but did not change in a dose-related manner.

An acute-duration inhalation MRL of $0.3 \mathrm{mg}$ boron $/ \mathrm{m}^{3}$ was derived using the NOAEL of $0.8 \mathrm{mg}$ boron $/ \mathrm{m}^{3}$ associated with a minimal LOAEL of $1.5 \mathrm{mg} / \mathrm{m}^{3}$ for increased nasal secretions in exercising volunteers. The NOAEL of $0.8 \mathrm{mg} / \mathrm{m}^{3}$ was divided by an uncertainty factor of $3\left(10^{0.5}\right.$ for human variability in the pharmacodynamic response to boron).

A series of studies conducted by Wilding et al. (1959) examined the toxicity of boron following intermediate-duration exposure of rats. No adverse effects, as assessed by a histological examination of a comprehensive set of tissues, were observed in rats exposed to aerosols of boron oxide (6 hours/day, 5 days/week) at concentrations of $73 \mathrm{mg}$ boron $/ \mathrm{m}^{3}$ for 10 weeks, $27 \mathrm{mg}$ boron $/ \mathrm{m}^{3}$ for 12 weeks, or $12 \mathrm{mg}$ boron $/ \mathrm{m}^{3}$ for 24 weeks. A reddish exudate from the nose was observed in some of the rats exposed to $73 \mathrm{mg}$ boron $/ \mathrm{m}^{3}$ for 10 weeks; the investigators noted that the rats were covered with dust and there probably was local irritation of the external nares and scratching. Another study by this group (Wilding et al. 1959) found no hematological alterations or alterations in sulfobromophthalein retention for liver damage in dogs exposed to $9 \mathrm{mg}$ boron $/ \mathrm{m}^{3}$ for 23 weeks. Because the NOAELs identified in the rat and dog studies were higher than concentrations associated with irritation in humans acutely exposed to boron 
(Cain et al. 2004, 2008; Wegman et al. 1994), the intermediate-duration inhalation database was considered inadequate for derivation of an MRL. However, these data do suggest that the acute-duration inhalation MRL of $0.01 \mathrm{mg}$ boron $/ \mathrm{m}^{3}$ should be health-protective for intermediate-duration exposures.

There are limited data on the chronic toxicity of boron in humans and no chronic-duration inhalation animal studies. Workers exposed to mean boron concentrations of 1.8 and $3.1 \mathrm{mg}$ boron $/ \mathrm{m}^{3}$ reported a higher frequency of respiratory symptoms such as dryness of the mouth, nose, or throat, dry cough, nose bleeds, and sore throat than in workers exposed to low levels of boron ( 0.9 and $0.2 \mathrm{mg}$ boron $/ \mathrm{m}^{3}$ ) (Garabrant et al. 1984, 1985); this is the same study population examined by Wegman et al. (1994) (see acute MRL discussion). No alterations in lung function, as measured by $\mathrm{FEV}_{1}$, were observed in nonsmoking workers; a reduced $\mathrm{FEV}_{1}$ was found in a subgroup of smoking workers with estimated boron exposure of $\geq 9 \mathrm{mg}$ boron $/ \mathrm{m}^{3}$. In the Wegman et al. (1994) follow-up study, no alterations in lung function were observed 7 years after the initial examination of workers receiving exposures of $\geq 15 \mathrm{mg}$ boron $/ \mathrm{m}^{3}$. The cross-sectional design of the Garabrant et al. (1985) study prevents determining whether the elevation of respiratory symptoms was a consequence of acute or repeated exposure to sodium borate dusts. Tarasenko et al. (1972) reported low sperm counts, reduced sperm motility, and elevated fructose content of seminal fluids in workers exposed to $22-80 \mathrm{mg} / \mathrm{m}^{3}$ boron aerosols (boron form uncertain) for $\geq 10$ years; however, interpretation of these results is limited by the small number of subjects and limited data reporting. Another study reported elevated fertility rates, as compared to U.S. national average, in workers employed at a borax production facility for at least 9 months (Whorton et al. 1994); no exposure data were reported. The uncertainty as to whether the effects observed in the Garabrant et al. (1984) study were due to acute or chronic exposure and the limitations in the Tarasenko et al. (1972) study preclude deriving a chronic-duration inhalation MRL for boron. However, the lack of chronic effects in workers observed by Wegman et al. (1994) 7 years after an assessment by Garabrant et al. (1985) suggests that the acute-duration inhalation MRL of $0.01 \mathrm{mg} / \mathrm{m}^{3}$ should be health-protective for chronicduration exposures.

\section{Oral MRLs}

- An MRL of $0.2 \mathrm{mg} / \mathrm{kg} /$ day has been derived for acute-duration oral exposure (1-14 days) to boron.

Acute-duration oral exposures of humans to high levels of boron (as boric acid) have resulted in little or no observable toxicity, as was seen in accidental poisonings of $10-88 \mathrm{~g}$, of which $88 \%$ of cases were asymptomatic (Litovitz et al. 1988). However, gastrointestinal, cardiovascular, hepatic, renal, and central 
nervous system effects, dermatitis, erythema, and death have been observed in children and adults exposed to $\geq 84 \mathrm{mg}$ boron/kg (Ishii et al. 1993; Restuccio et al. 1992; Schillinger et al. 1982; Wong et al. 1964).

Most of the available animal studies on the acute toxicity of boron have focused on developmental and reproductive toxicity end points. NTP (1987; Dieter 1994) reported gastric hyperplasia and dysplasia in mice exposed to 2,251 mg boron/kg/day as boric acid in the diet for 14 days; no gastrointestinal effects were observed at $926 \mathrm{mg}$ boron/kg/day. Similarly, Weir and Fisher (1972) reported vomiting in dogs receiving a single gavage dose of $1,000 \mathrm{mg}$ boron/kg/day as boric acid. Testicular and spermatogenic effects were observed in rats receiving gavage doses of $88 \mathrm{mg}$ boron/kg/day for 2 weeks (Fukuda et al. 2000; Kudo et al. 2000). No effects were observed at 44 or $53 \mathrm{mg}$ boron/kg/day.

A series of studies conducted by Cherrington and Chernoff (2002) demonstrate the fetal toxicity of boron in mice. A variety of skeletal malformations (e.g., rib agenesis, fused rib, cervical rib, reduced rib length) were observed in the fetuses of mice receiving gavage doses of $88 \mathrm{mg}$ boron $/ \mathrm{kg} /$ day on gestation days 6$10,131 \mathrm{mg}$ boron/kg on gestation day 8 , or $70 \mathrm{mg}$ boron/kg administered twice daily on gestation day 8 or 6-8. Two gavage doses of $131 \mathrm{mg}$ boron $/ \mathrm{kg}$ on gestation day 8 resulted in multiple thoracic skeletal malformations. Reductions in fetal body weights were also observed in these studies and in studies of mice receiving two gavage doses of $70 \mathrm{mg}$ boron/ $/ \mathrm{kg}$ on gestation days 6, 7, 9, or 10 . However, skeletal malformations were not observed in studies that did not include exposure on gestation day 8. No NOAELs for developmental effects were observed in the Cherrington and Chernoff (2002) studies. A study of rabbits (Price et al. 1996b) identified a lower LOAEL for developmental toxicity. At gavage doses of $44 \mathrm{mg}$ boron/kg/day as boric acid administered on gestation days $6-19$, significant increases in resorptions and decreases in the number of live litters and fetuses were observed. This dose was also associated with decreases in fetal body weight and increases (on percent fetuses per litter basis) in external, visceral, and cardiovascular malformations. Marked decreases in maternal body weight were also observed at $44 \mathrm{mg}$ boron/kg/day. No adverse maternal or fetal effects were observed at $22 \mathrm{mg}$ boron/kg/day.

The Price et al. (1996b) study was selected as the principal study for derivation of an acute-duration oral MRL because it identified a lower LOAEL than the Cherrington and Chernoff (2002) studies and involved a longer duration of exposure (14 days compared to 5 days). In the Price et al. (1996b) study, groups of 30 pregnant New Zealand white rabbits were given gavage doses of 0, 62.5, 125, or $250 \mathrm{mg}$ boric acid/kg/day $(0,11,22$, or $44 \mathrm{mg}$ boron/kg/day) on gestation days 6-19. Observations were made 
for clinical signs, maternal and fetal body weight, number of implantations, resorptions, number of live and dead fetuses, and fetal external, visceral, and skeletal defects. No adverse maternal effects were observed in rabbits in the 11 or $22 \mathrm{mg}$ boron/kg/day groups. At $44 \mathrm{mg}$ boron/kg/day, decreases in maternal body weight, relative kidney weight, and food consumption were observed. During the treatment period, the rabbits lost $137 \mathrm{~g}$ body weight compared to a weight gain of $93 \mathrm{~g}$ in controls. No differences in the number of implantation sites per litter were observed; however, there were significant increases in the percent resorptions per litter, percent of litters with one or more resorptions, and percent of litters with $100 \%$ resorption. The number of live litters was 18, 23, 20, and 6 in the 0,11 , 22, and $44 \mathrm{mg}$ boron/kg/day groups, respectively, and the number of live fetuses was 159, 175, 153, and 14, respectively. A decrease in fetal body weights (92\% of controls) was observed at $44 \mathrm{mg}$ boron/kg/day; although the body weight was not significantly different from controls, the effect was considered biologically significant. Significant increases in the percent of fetuses per litter with external, visceral, and cardiovascular malformations and cardiovascular variations were observed. Although the overall incidence of external malformations was increased at $44 \mathrm{mg}$ boron/ $\mathrm{kg} /$ day, there were no increases in a specific malformation. The visceral malformations primarily consisted of cardiovascular malformations, particularly interventricular septal defect, enlarged aorta, papillary muscle malformation, and double outlet right ventricle. The cardiovascular variations consisted of abnormal number of cardiac papillary muscles.

An acute-duration oral MRL of $0.2 \mathrm{mg}$ boron/ $/ \mathrm{kg} / \mathrm{day}$ was derived using the NOAEL of $22 \mathrm{mg}$ boron/kg/day associated with a LOAEL of $44 \mathrm{mg}$ boron/kg/day for increased incidence of external, visceral, and cardiovascular malformations and reduced body weight in the fetuses of rabbits administered boric acid via gavage on gestation days 6-19. The NOAEL of $22 \mathrm{mg}$ boron/kg/day was divided by an uncertainty factor of 100 (10 for interspecies extrapolation and 10 for human variability).

- An MRL of $0.2 \mathrm{mg} / \mathrm{kg} /$ day has been derived for intermediate-duration oral exposure (15364 days) to boron.

There are limited data on the intermediate-duration toxicity of boron in humans. Seizure disorders were observed in infants orally exposed to approximately 12-120 g of borax for 4-12 weeks (Gordon et al. 1973; O'Sullivan and Taylor 1983). The possible association between boron exposure and impaired fertility was investigated in Turkish subpopulations expected to have intermediate- to chronic-duration exposures to boron (Sayli 1998a, 1998b, 2003; Sayli et al. 1998) and boron mining and processing workers, which may have included oral exposure to boron (Chang et al. 2006; Whorton et al. 1994). These studies did not find significant associations. 
Animal studies have clearly identified reproductive and developmental toxicity as the most sensitive effects of oral boron exposure. Intermediate-duration exposure of rats, mice, and dogs to boric acid or borax results in histological damage to the testes and the associated impacts on spermatogenesis (sperm abnormalities and reduced sperm production) at doses $\geq 26 \mathrm{mg}$ boron/kg/day as boric acid (Dieter 1994; Dixon et al. 1976, 1979; Fail et al. 1991; Fukuda et al. 2000; Harris et al. 1992; Ku et al. 1993a; Kudo et al. 2000; Lee et al. 1978; NTP 1987; Nusier and Bataineh 2005; Seal and Weeth 1980; Weir and Fisher 1972; Yoshizaki et al. 1999). Complete sterility was observed in rats exposed to $101 \mathrm{mg}$ boron/kg/day as boric acid or borax for 14 weeks prior to mating (Weir and Fisher 1972); a lack of viable sperm was observed at this dose level. Additionally, female rats exposed to similar doses (116 mg boron/kg/day) for 14 weeks failed to become pregnant when mated with non-exposed males (Weir and Fisher 1972); the female sterility response at this dose level was associated with decreased ovulation. The Weir and Fisher (1972) 3-generation studies (males and females exposed to boric acid or borax) established a NOAEL of $30 \mathrm{mg}$ boron/kg/day for reproductive toxicity in rats.

The developing fetus appears to be a more sensitive target than the reproductive system. Reductions in fetal body weights were observed in rats following exposure to $13-13.6 \mathrm{mg}$ boron/ $\mathrm{kg} /$ day as boric acid on gestation days 0-20 (Heindel et al. 1992; Price et al. 1996a); an increase in the occurrence of skeletal abnormalities was also observed at this dose level (Price et al. 1996a). At $28.4 \mathrm{mg}$ boron/kg/day, rib cage defects, enlargement of the lateral ventricles of the brain, and increased resorptions were observed in rats exposed to boric acid on gestation days 0-20 (Heindel et al. 1992). No developmental effects were observed in rats exposed to $10 \mathrm{mg}$ boron/kg/day as boric acid on gestation days 0-20 (Price et al. 1996a). In mice, gestational exposure on days 0-17 resulted in reduced fetal weights at $79 \mathrm{mg}$ boron/ $\mathrm{kg} / \mathrm{day}$ and increased skeletal defects and increased resorptions at $175.3 \mathrm{mg}$ boron/kg/day (Heindel et al. 1992); a NOAEL of $43.4 \mathrm{mg}$ boron/kg/day was identified.

Systemic effects are observed at somewhat higher doses. Hematological alterations (splenic extramedullary hematopiesis and decreased hemoglobin levels) were observed at 60.5 and $72 \mathrm{mg}$ boron/kg/day in dogs and rats, respectively, exposed to as borax or boric acid (NTP 1987; Weir and Fisher 1972), desquamation of paw and tail skin and eye inflammation were observed in rats exposed to $150 \mathrm{mg}$ boron/kg/day as boric acid or borax (Weir and Fisher 1972), and hyperkeratosis and/or acanthosis was observed in rats at $577 \mathrm{mg}$ boron/kg/day as boric acid (NTP 1987). 
The available intermediate-duration oral database clearly identifies the developing fetus as the most sensitive target of toxicity. Two studies in rats (Heindel et al. 1992; Price et al. 1996a) identified LOAELs of 13-13.6 mg boron/kg/day for decreases in fetal body weight and skeletal malformations (only identified in the Price et al. 1996a study). These LOAELs are lower than the NOAEL of $30 \mathrm{mg}$ boron/kg/day identified for reproductive toxicity in a 3-generation study (Weir and Fisher 1972) and NOAELs of 35 or $45 \mathrm{mg}$ boron/kg/day for hematological and dermal effects (Weir and Fisher 1972).

Multiple developmental end point data from the Price et al. (1996a) and Heindel et al. (1992) studies were pooled and subjected to multiple benchmark dose analyses (Allen et al. 1996); see Appendix A for summaries of these two studies and the benchmark dose analysis. The 95\% lower confidence limit on the benchmark dose associated with a $5 \%$ reduction in fetal body weight $\left(\mathrm{BMDL}_{05}\right)$ was calculated to be $10.3 \mathrm{mg}$ boron/kg/day. This estimate was similar to the observed NOAEL of $10 \mathrm{mg}$ boron $/ \mathrm{kg} /$ day (Price et al. 1996a) and was used as a point of departure for derivation of the intermediate-duration oral MRL. The $\mathrm{BMDL}_{05}$ of $10.3 \mathrm{mg}$ boron/kg/day was divided by a chemical-specific uncertainty factor of 66 (3.3 for toxicokinetic extrapolation from animals to humans, 3.16 for toxicodynamic extrapolation from animals to humans, 2.0 for variability in human toxicokinetics, and 3.16 for variability in human toxicodynamics) (see Appendix A for derivation of the chemical-specific uncertainty factor) resulting in an intermediate-duration oral MRL of $0.2 \mathrm{mg}$ boron/kg/day.

\subsubsection{Chronic-Duration Oral Studies}

As previously discussed, no significant associations between boron exposure and impaired fertility were observed in Turkish subpopulations expected to have intermediate- to chronic-duration exposures to boron (Sayli 1998a, 1998b; Sayli et al. 1998, 2003). Chronic-duration studies have been conducted in rats and dogs exposed to boric acid or borax in the diet (Weir and Fisher 1972) and mice exposed to boric acid in the diet (Dieter 1994; NTP 1987). Systemic effects consisted of hematological alterations (decreases in hemoglobin in rats and splenic hematopoeisis in mice), desquamation of footpad skin and bloody ocular discharge in rats, decreased body weight gain in rats and mice, lung hemorrhage in mice, and hepatic chronic inflammation and coagulative necrosis in mice. The hematological, dermal, ocular, and body weight effects were observed in rats exposed to $81 \mathrm{mg}$ boron/kg/day (NOAEL of $24 \mathrm{mg}$ boron/kg/day). In mice, the hematological and liver effects were observed at $79 \mathrm{mg}$ boron/kg/day and the body weight and lung effects were observed at $201 \mathrm{mg}$ boron/kg/day. The highest dose tested in the dog studies (6.8 mg boron/kg/day) was a NOAEL for systemic effects. Testicular atrophy was observed in rats exposed to $81 \mathrm{mg}$ boron/kg/day as boric acid or borax (Weir and Fisher 1972) and mice exposed to 
$201 \mathrm{mg}$ boron/kg/day as boric acid (Dieter 1994; NTP 1987); the NOAELs for these effects were 24 and $79 \mathrm{mg}$ boron/kg/day for the rats and mice, respectively. A chronic-duration oral MRL, based on results from the chronic oral toxicity studies in animals, was not derived. However, the intermediate MRL, which is based on developmental toxicity, should be protective for chronic exposure because the NOAEL (24 mg boron/kg/day) for testicular atrophy and systemic effects in chronically exposed rats (Weir and Fisher 1972) was higher than the intermediate-duration LOAELs of 13-13.6 mg boron/kg/day for developmental toxicity in rats (Heindel et al. 1992; Price et al. 1996a). 
2. RELEVANCE TO PUBLIC HEALTH

This page is intentionally blank. 


\section{HEALTH EFFECTS}

\subsection{INTRODUCTION}

The primary purpose of this chapter is to provide public health officials, physicians, toxicologists, and other interested individuals and groups with an overall perspective on the toxicology of boron. It contains descriptions and evaluations of toxicological studies and epidemiological investigations and provides conclusions, where possible, on the relevance of toxicity and toxicokinetic data to public health.

A glossary and list of acronyms, abbreviations, and symbols can be found at the end of this profile.

\subsection{DISCUSSION OF HEALTH EFFECTS BY ROUTE OF EXPOSURE}

To help public health professionals and others address the needs of persons living or working near hazardous waste sites, the information in this section is organized first by route of exposure (inhalation, oral, and dermal) and then by health effect (death, systemic, immunological, neurological, reproductive, developmental, genotoxic, and carcinogenic effects). These data are discussed in terms of three exposure periods: acute (14 days or less), intermediate (15-364 days), and chronic (365 days or more).

Levels of significant exposure for each route and duration are presented in tables and illustrated in figures. The points in the figures showing no-observed-adverse-effect levels (NOAELs) or lowestobserved-adverse-effect levels (LOAELs) reflect the actual doses (levels of exposure) used in the studies. LOAELs have been classified into "less serious" or "serious" effects. "Serious" effects are those that evoke failure in a biological system and can lead to morbidity or mortality (e.g., acute respiratory distress or death). "Less serious" effects are those that are not expected to cause significant dysfunction or death, or those whose significance to the organism is not entirely clear. ATSDR acknowledges that a considerable amount of judgment may be required in establishing whether an end point should be classified as a NOAEL, "less serious" LOAEL, or "serious" LOAEL, and that in some cases, there will be insufficient data to decide whether the effect is indicative of significant dysfunction. However, the Agency has established guidelines and policies that are used to classify these end points. ATSDR believes that there is sufficient merit in this approach to warrant an attempt at distinguishing between "less serious" and "serious" effects. The distinction between "less serious" effects and "serious" effects is considered to be important because it helps the users of the profiles to identify levels of exposure at which major health effects start to appear. LOAELs or NOAELs should also help in determining whether or not 
the effects vary with dose and/or duration, and place into perspective the possible significance of these effects to human health.

The significance of the exposure levels shown in the Levels of Significant Exposure (LSE) tables and figures may differ depending on the user's perspective. Public health officials and others concerned with appropriate actions to take at hazardous waste sites may want information on levels of exposure associated with more subtle effects in humans or animals (LOAELs) or exposure levels below which no adverse effects (NOAELs) have been observed. Estimates of levels posing minimal risk to humans (Minimal Risk Levels or MRLs) may be of interest to health professionals and citizens alike.

A User's Guide has been provided at the end of this profile (see Appendix B). This guide should aid in the interpretation of the tables and figures for Levels of Significant Exposure and the MRLs.

\subsubsection{Inhalation Exposure}

Most of the inhalation toxicity data for boron involves human or animal exposure to borate dusts such as boric acid, boron oxide, or various hydration states of sodium borate salts (anhydrous; pentahydrate; and decahydrate; also referred to as borax) or other borate salts (e.g., calcium borate). In aqueous media, boron oxide is rapidly transformed to boric acid and, depending on the $\mathrm{pH}$ of the media, to borate salts. Equivalent boron doses have been calculated throughout this assessment by multiplying reported doses by an adjustment factor relating the molar mass ratio of boron in a given compound to the total molar mass. Thus, the dose adjustment factors are 0.175 for boric acid, 0.113 for disodium borate decahydrate, 0.151 for disodium borate pentahydrate, 0.215 for anhydrous borate, and 0.155 for boron oxide. In addition, animal studies were located reporting the effects of inhaled diborane, a flammable boroncontaining gas. Because of the differences in environmental prevalence, industrial uses, likelihood of exposure, and respiratory toxicity, the effects of diborane exposure are discussed separately from other boron compounds in Section 3.2.1.2, Respiratory Effects.

\subsubsection{Death}

No studies were located regarding death in humans after inhalation exposure to boron. The 4-hour $\mathrm{LC}_{50}$ for boric acid, borax, and disodium borates is $>2 \mathrm{mg}$ boron $/ \mathrm{m}^{3}$ (Hubbard 1998). No fatalities were observed in rats exposed for 6 hours/day, 5 days/week, to $470 \mathrm{mg}$ boron oxide $/ \mathrm{m}^{3}$ (73 $\mathrm{mg}$ boron $/ \mathrm{m}^{3}$ ) for 10 weeks, $175 \mathrm{mg}$ boron oxide $/ \mathrm{m}^{3}$ (27 mg boron $/ \mathrm{m}^{3}$ ) for 12 weeks, or $77 \mathrm{mg}$ boron oxide $/ \mathrm{m}^{3}$ (12 mg 
boron $/ \mathrm{m}^{3}$ ) for 24 weeks, or dogs exposed to $57 \mathrm{mg}$ boron oxide $/ \mathrm{m}^{3}$ ( $9 \mathrm{mg}$ boron $/ \mathrm{m}^{3}$ ) for 23 weeks (Wilding et al. 1959).

\subsubsection{Systemic Effects}

No studies were located regarding cardiovascular, gastrointestinal, hematological, musculoskeletal, or renal effects in humans after inhalation exposure to boron. No studies were located regarding dermal effects after acute inhalation exposure in humans or animals for any duration category, but eye irritation has been reported in sodium-borate mining and processing workers. Information on respiratory, cardiovascular, gastrointestinal, hematological, musculoskeletal, and renal effects in animals and respiratory effects and dermal/ocular effects in humans is discussed below. The highest NOAEL values and all reliable LOAEL values for these systemic effects for each species and duration category involving exposure to boric acid, borates, or boron oxide are recorded in Table 3-1 and plotted in Figure 3-1. Results from a few mouse inhalation studies of diborane gas indicate that it is a potent respiratory toxicant, much more potent than borates or boron oxide. Since it is not expected to be as important an environmental compound as borates or boron oxide, NOAEL and LOAEL values from these mouse studies of diborane gas are not included in Table 3-1 or Figure 3-1.

Respiratory Effects. Occupational studies of workers exposed to dusts of sodium borates, the most important commercial forms of boron, have identified irritation of the respiratory tract and eyes, without measurable changes in pulmonary function. In an early cross-sectional surveillance of 629 U.S. workers in a sodium borate open-pit mining and production plant, past occurrence of symptoms of respiratory irritation such as dryness of the mouth, nose, or throat, dry cough, nose bleeds, and sore throat were reported at elevated frequencies in workers in areas with mean dust concentrations of 8.4 and $14.6 \mathrm{mg}$ particulates $/ \mathrm{m}^{3}$ (1.8 and $3.1 \mathrm{mg}$ boron $/ \mathrm{m}^{3}$, respectively), compared with workers in areas with lower mean dust levels of 4.0 and $1.1 \mathrm{mg}$ particulate $/ \mathrm{m}^{3}$ (0.9 and $0.2 \mathrm{mg}$ boron $/ \mathrm{m}^{3}$ ) (Garabrant et al. 1984; 1985). These boron concentrations are upper bound estimates, assuming that all of the sampled dust is anhydrous borax. A reduction in forced expiratory volume in 1 second $\left(\mathrm{FEV}_{1}\right)$ was measured in a subgroup of smoking workers with estimated high cumulative exposure ( $\geq 80 \mathrm{mg}$ particulate $/ \mathrm{m}^{3}, \geq 9 \mathrm{mg}$ boron $/ \mathrm{m}^{3}$ ) to sodium borate dusts, but not in groups of less-exposed smoking workers or in nonsmoking workers. However, a subsequent surveillance of $\mathrm{FEV}_{1}$ in 303 of the original 629 borax workers, 7 years after the

original surveillance, , some of whom were exposed to $\geq 15 \mathrm{mg}$ boron $/ \mathrm{m}^{3}$, found no exposure-related changes in $\mathrm{FEV}_{1}$ over this period, when adjustments were made for the effects of age, height, and smoking on $\mathrm{FEV}_{1}$ (Wegman et al. 1991, 1994). Although the prevalence of workers reporting acute 
Table 3-1 Levels of Significant Exposure to Boron - Inhalation

\begin{tabular}{|c|c|c|c|c|c|c|c|c|}
\hline \multirow[b]{2}{*}{$\begin{array}{l}\text { Key to } \\
\text { Figure }\end{array}$} & \multirow[b]{2}{*}{$\begin{array}{c}\text { Species } \\
\text { (Strain) }\end{array}$} & \multirow{2}{*}{$\begin{array}{c}\text { Exposurel } \\
\text { Duration/ } \\
\text { Frequency } \\
\text { (Route) }\end{array}$} & \multirow[b]{2}{*}{ System } & \multirow[b]{2}{*}{$\begin{array}{l}\text { NOAEL } \\
\left(\mathrm{mg} / \mathrm{m}^{3}\right)\end{array}$} & \multicolumn{2}{|c|}{ LOAEL } & \multirow[b]{2}{*}{$\begin{array}{l}\text { Reference } \\
\text { Chemical Form }\end{array}$} & \multirow[b]{2}{*}{ Comments } \\
\hline & & & & & $\begin{array}{c}\text { Less Serious } \\
\qquad\left(\mathrm{mg} / \mathrm{m}^{3}\right)\end{array}$ & $\begin{array}{l}\text { Serious } \\
\left(\mathrm{mg} / \mathrm{m}^{3}\right)\end{array}$ & & \\
\hline
\end{tabular}

ACUTE EXPOSURE

Systemic

1 Human

once

Resp

$0.8 \mathrm{M}$

1.5 M (mild irritation of nose

and throat; increased

nasal secretion)

Cain et al. 2004

SODIUM BORATE

2 Human

$1 \times 47 \mathrm{~min}$

Resp

0.9

1.8

(increased nasa

secretions)

Cain et al. 2008

BORIC ACID

\section{INTERMEDIATE EXPOSURE}

\section{Systemic}

3 Rat

$10 \mathrm{wk}$

$5 \mathrm{~d} / \mathrm{wk}$

Resp

73 (reddish nasal exudate)

Wilding et al. 1959

Negative

BORIC OXIDE

histopathology on all

examined tissues.

$\begin{array}{ll}\text { Cardio } & 73 \\ \text { Gastro } & 73 \\ \text { Hemato } & 73 \\ \text { Musc/skel } & 73 \\ \text { Hepatic } & 73 \\ \text { Renal } & 73 \\ \text { Endocr } & 73 \\ \text { Ocular } & 73 \\ \text { Bd Wt } & 73\end{array}$


Table 3-1 Levels of Significant Exposure to Boron - Inhalation

(continued)

\begin{tabular}{|c|c|c|c|c|c|c|c|c|}
\hline \multirow[b]{2}{*}{$\begin{array}{l}\text { Key to } \\
\text { Figure } \\
\end{array}$} & \multirow[b]{2}{*}{$\begin{array}{l}\text { Species } \\
\text { (Strain) }\end{array}$} & \multirow{2}{*}{$\begin{array}{l}\text { Exposurel } \\
\text { Duration/ } \\
\text { Frequency } \\
\text { (Route) }\end{array}$} & \multirow[b]{2}{*}{ System } & \multirow[b]{2}{*}{$\begin{array}{l}\text { NOAEL } \\
\left(\mathrm{mg} / \mathrm{m}^{3}\right)\end{array}$} & \multicolumn{2}{|c|}{ LOAEL } & & \multirow[b]{2}{*}{ Comments } \\
\hline & & & & & $\begin{array}{c}\text { Less Serious } \\
\left(\mathrm{mg} / \mathrm{m}^{3}\right)\end{array}$ & $\begin{array}{l}\text { Serious } \\
\left(\mathrm{mg} / \mathrm{m}^{3}\right)\end{array}$ & $\begin{array}{l}\text { Reference } \\
\text { Chemical Form }\end{array}$ & \\
\hline \multirow[t]{10}{*}{4} & Rat & $\begin{array}{l}12 \mathrm{wk} \\
5 \mathrm{~d} / \mathrm{wk} \\
6 \mathrm{hr} / \mathrm{d}\end{array}$ & Resp & 27 & & & $\begin{array}{l}\text { Wilding et al. } 1959 \\
\text { BORIC OXIDE }\end{array}$ & $\begin{array}{l}\text { NOAELs are for } \\
\text { histopathology or } \\
\text { hematology. }\end{array}$ \\
\hline & & & Cardio & 27 & & & & \\
\hline & & & Gastro & 27 & & & & \\
\hline & & & Hemato & 27 & & & & \\
\hline & & & Musc/skel & 27 & & & & \\
\hline & & & Hepatic & 27 & & & & \\
\hline & & & Renal & 27 & & & & \\
\hline & & & Endocr & 27 & & & & \\
\hline & & & Ocular & 27 & & & & \\
\hline & & & $\mathrm{Bd} W \mathrm{t}$ & 27 & & & & \\
\hline
\end{tabular}


Table 3-1 Levels of Significant Exposure to Boron - Inhalation

\begin{tabular}{|c|c|c|c|c|}
\hline $\begin{array}{l}\text { Key to } \\
\text { Figure }\end{array}$ & $\begin{array}{l}\text { Species } \\
\text { (Strain) }\end{array}$ & $\begin{array}{c}\text { Exposurel } \\
\text { Duration/ } \\
\text { Frequency } \\
\text { (Route) }\end{array}$ & System & $\begin{array}{l}\text { NOAE } \\
(\mathrm{mg} / \mathrm{r}\end{array}$ \\
\hline \multirow[t]{10}{*}{5} & Rat & $\begin{array}{l}24 \mathrm{wk} \\
5 \mathrm{~d} / \mathrm{wk} \\
6 \mathrm{hr} / \mathrm{d}\end{array}$ & Resp & 12 \\
\hline & & & Cardio & 12 \\
\hline & & & Gastro & 12 \\
\hline & & & Hemato & 12 \\
\hline & & & Musc/skel & 12 \\
\hline & & & Hepatic & 12 \\
\hline & & & Renal & 12 \\
\hline & & & Endocr & 12 \\
\hline & & & Ocular & 12 \\
\hline & & & $\mathrm{Bd} \mathrm{Wt}$ & 2 \\
\hline
\end{tabular}

LOAEL

Reference

Chemical Form

Comments

6 Dog 23 wk Hemato 9

Wilding et al. 1959

BORIC OXIDE

Hematology and serum chemistry values

comparable to controls; no histological exams reported.

Hepatic 9

Immunol Lymphoret

$7 \quad$ Rat $\quad 12 \mathrm{wk}$

(albino) $\quad 5 \mathrm{~d} / \mathrm{wk}$

Wilding et al. 1959

NOAEL is for lymph

node and spleen

BORIC OXIDE

NOAELS are for

histopathology or

hematology. 
Table 3-1 Levels of Significant Exposure to Boron - Inhalation

\begin{tabular}{|c|c|}
\hline $\begin{array}{l}\text { Key to } \\
\text { Figure }\end{array}$ & $\begin{array}{c}\text { Species } \\
\text { (Strain) }\end{array}$ \\
\hline 8 & $\begin{array}{l}\text { Rat } \\
\text { (albino) }\end{array}$ \\
\hline
\end{tabular}

(albino) $\quad 5 \mathrm{~d} / \mathrm{w}$

$9 \quad$ Rat

(albino) $\begin{aligned} & 5 \mathrm{~d} / \mathrm{wk} \\ & 6 \mathrm{hr} / \mathrm{d}\end{aligned}$

Neurological

10 Rat

(albino) $\quad \begin{array}{ll}10 \mathrm{wk} \\ 5 \mathrm{~d} / \mathrm{wk}\end{array}$

$5 \mathrm{~d} / \mathrm{wk}$
$6 \mathrm{hr} / \mathrm{d}$

73

11 Rat $12 \mathrm{wk}$

(albino) $\quad 5 \mathrm{~d} / \mathrm{wk}$

$6 \mathrm{hr} / \mathrm{d}$

12 Rat 24 wk

$5 \mathrm{~d} / \mathrm{wk}$

$6 \mathrm{hr} / \mathrm{d}$

\section{Reproductive}

13 Rat

$12 \mathrm{wk}$

$5 \mathrm{~d} / \mathrm{wk}$

$6 \mathrm{hr} / \mathrm{d}$

14 Rat

(albino) $\quad 5 \mathrm{~d} / \mathrm{wk}$

LOAEL

Reference

Chemical Form

Comments

$\left(\mathrm{mg} / \mathrm{m}^{3}\right)$

Wilding et al. 1959

BORIC OXIDE

NOAEL is for lymph

node and spleen

histopathology.

Wilding et al. 1959

Negative lymph node and spleen

histopathology.

BORIC OXIDE

Wilding et al. 1959

NOAEL is for brain histopathology.

BORIC OXIDE

NOAEL is for brain

histopathology.

BORIC OXIDE

Wilding et al. 1959

BORIC OXIDE

NOAEL is for brain

histopathology.

Wilding et al. 1959

NOAEL is for testes or

BORIC OXIDE

ovary histopathology.

Wilding et al. 1959

NOAEL is for testes or

BORIC OXIDE ovary histopathology. 
Table 3-1 Levels of Significant Exposure to Boron - Inhalation

(continued)

\begin{tabular}{|c|c|c|c|c|c|c|c|c|c|}
\hline \multirow[b]{2}{*}{$\begin{array}{l}\text { Key to } \\
\text { Figure } \\
\end{array}$} & \multirow[b]{2}{*}{$\begin{array}{l}\text { Species } \\
\text { (Strain) }\end{array}$} & \multirow{2}{*}{$\begin{array}{l}\text { Exposure/ } \\
\text { Duration/ } \\
\text { Frequency } \\
\text { (Route) }\end{array}$} & \multirow[b]{2}{*}{ System } & \multirow[b]{2}{*}{$\begin{array}{l}\text { NOAEL } \\
\left(\mathrm{mg} / \mathrm{m}^{3}\right) \\
\end{array}$} & \multicolumn{3}{|c|}{ LOAEL } & \multirow[b]{2}{*}{$\begin{array}{l}\text { Reference } \\
\text { Chemical Form }\end{array}$} & \multirow[b]{2}{*}{ Comments } \\
\hline & & & & & Les & $\begin{array}{l}\text { Serious } \\
\left.\mathrm{ng} / \mathrm{m}^{3}\right)\end{array}$ & $\begin{array}{l}\text { Serious } \\
\left(\mathrm{mg} / \mathrm{m}^{3}\right)\end{array}$ & & \\
\hline 15 & Rat & $\begin{array}{l}10 \mathrm{wk} \\
5 \mathrm{~d} / \mathrm{wk} \\
6 \mathrm{hr} / \mathrm{d}\end{array}$ & & 73 & & & & $\begin{array}{l}\text { Wilding et al. } 1959 \\
\text { BORIC OXIDE }\end{array}$ & $\begin{array}{l}\text { NOAEL is for testes o } \\
\text { ovary histopathology. }\end{array}$ \\
\hline \multicolumn{10}{|c|}{$\begin{array}{l}\text { CHRONIC EXPOSURE } \\
\text { Systemic }\end{array}$} \\
\hline 16 & Human & 11.4 yr (mean) & Resp & & 1.8 & $\begin{array}{l}\text { (dryness } \\
\text { nose, or } \\
\text { cough, n } \\
\text { sore thro }\end{array}$ & & $\begin{array}{l}\text { Garabrant et al. } 1984,1985 \\
\text { SODIUM BORATE }\end{array}$ & $\begin{array}{l}\text { Uncertain whether } \\
\text { symptoms were from } \\
\text { acute or chronic } \\
\text { exposures. }\end{array}$ \\
\hline
\end{tabular}

a The number corresponds to entries in Figure 3-1.

b Used to derive an acute-duration inhalation MRL of $0.3 \mathrm{mg}$ boron $/ \mathrm{m}^{3}$; exposure level divided by an uncertainty factor of 3 (10.5 for human pharmacodynamic variability).

$\mathrm{Bd} \mathrm{Wt}=$ body weight; Cardio = cardiovascular; $\mathrm{d}=$ day(s); Endocr = endocrine; $\mathrm{F}=$ Female; Gastro = gastrointestinal; Hemato = hematological; hr = hour(s); Immuno/Lymphoret =

immunological/lymphoreticular; LOAEL = lowest-observed-adverse-effect level; $\mathrm{M}=$ male; $\min =$ minute(s); Musc/skel = musculoskeletal; NOAEL = no-observed-adverse-effect level;

NS = not specified; occup = occupational; Resp = respiratory; TWA = time-weighted average; $x=$ time $(s) ;$ wk = week $(s) ; y r=y e a r(s)$ 
Figure 3-1 Levels of Significant Exposure to Boron - Inhalation

Acute ( $\leq 14$ days)

\section{Systemic}

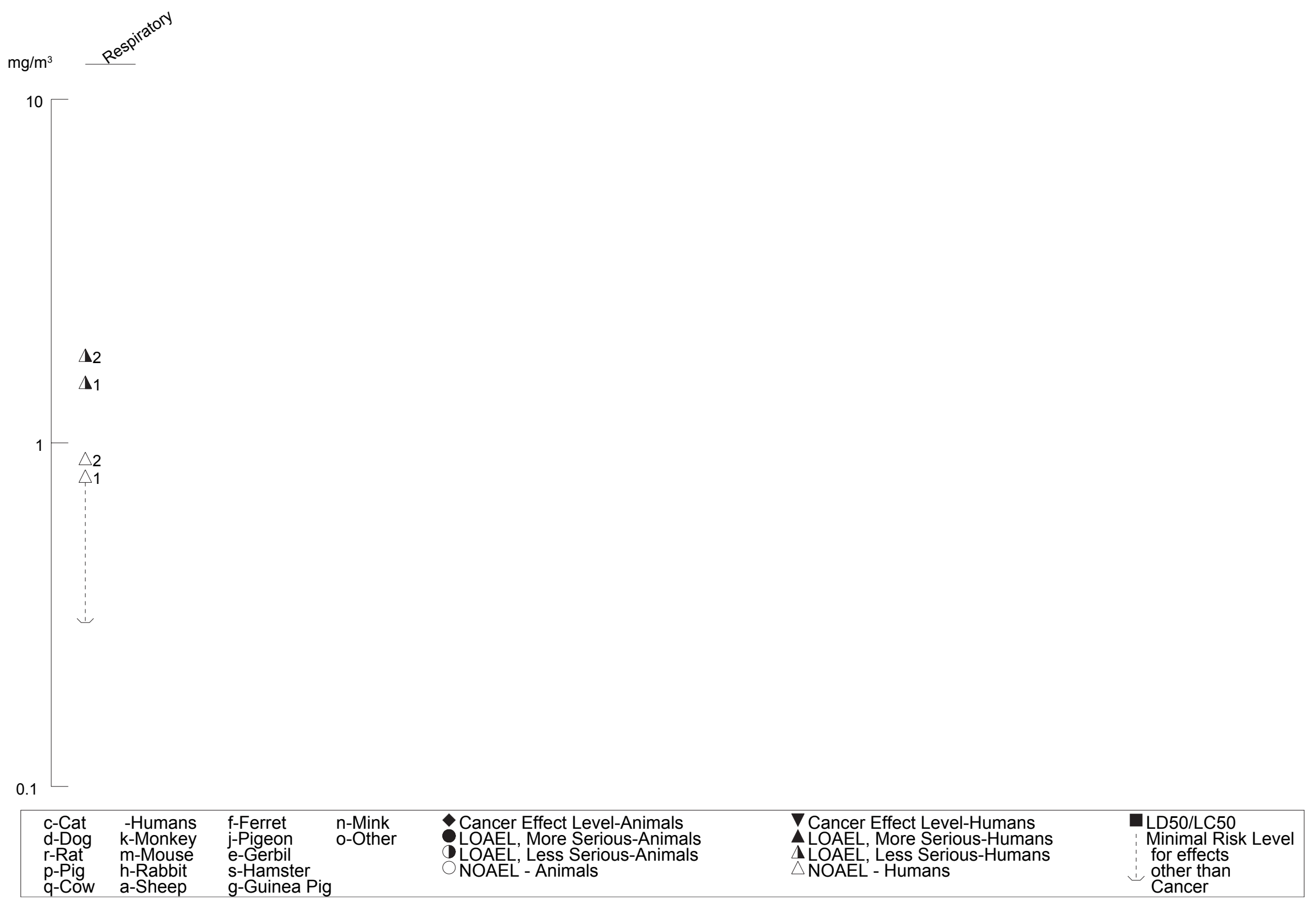


Figure 3-1 Levels of Significant Exposure to Boron - Inhalation (Continued)

Intermediate (15-364 days)

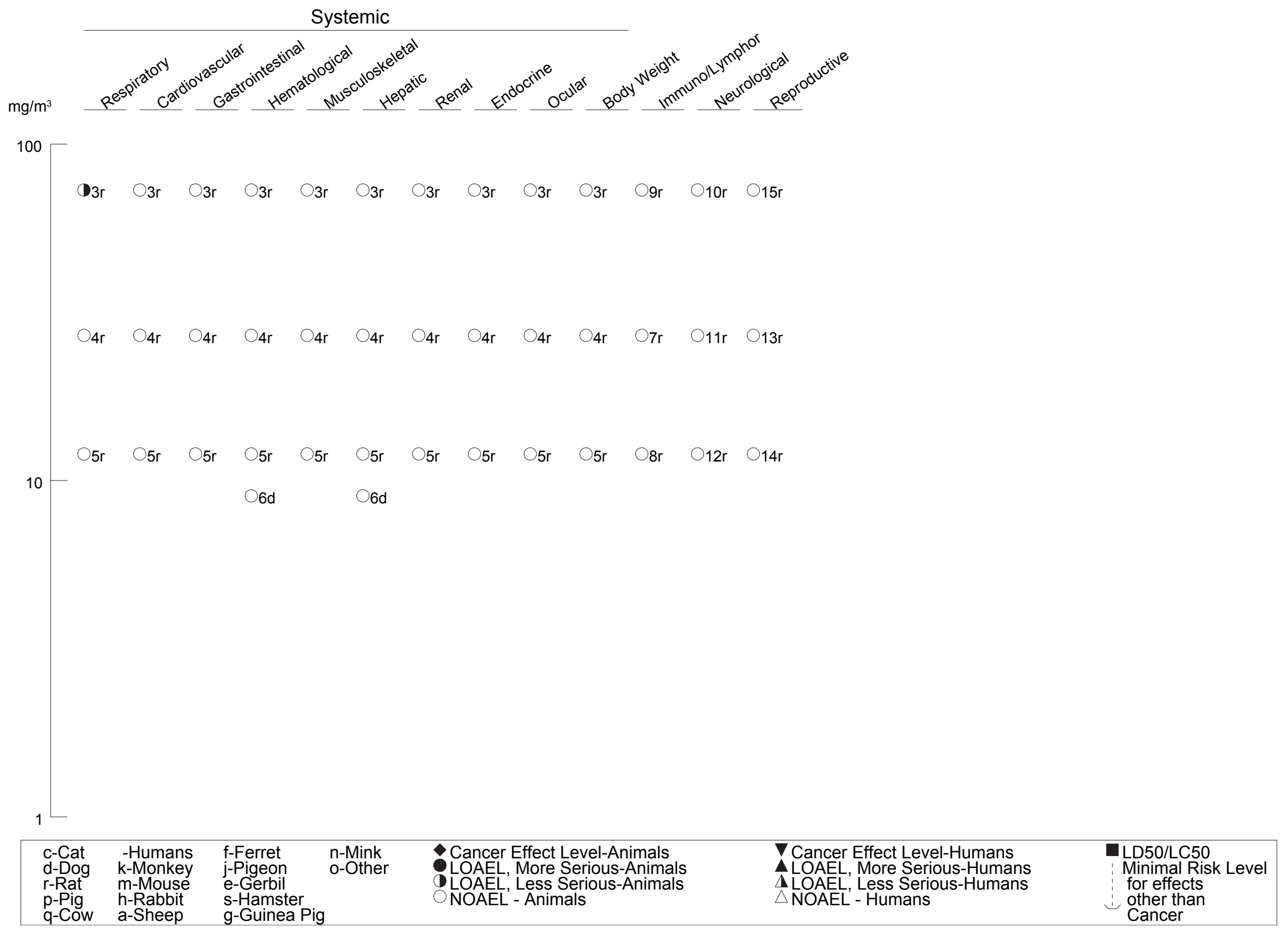


Figure 3-1 Levels of Significant Exposure to Boron - Inhalation (Continued)

Chronic ( $\geq 365$ days)

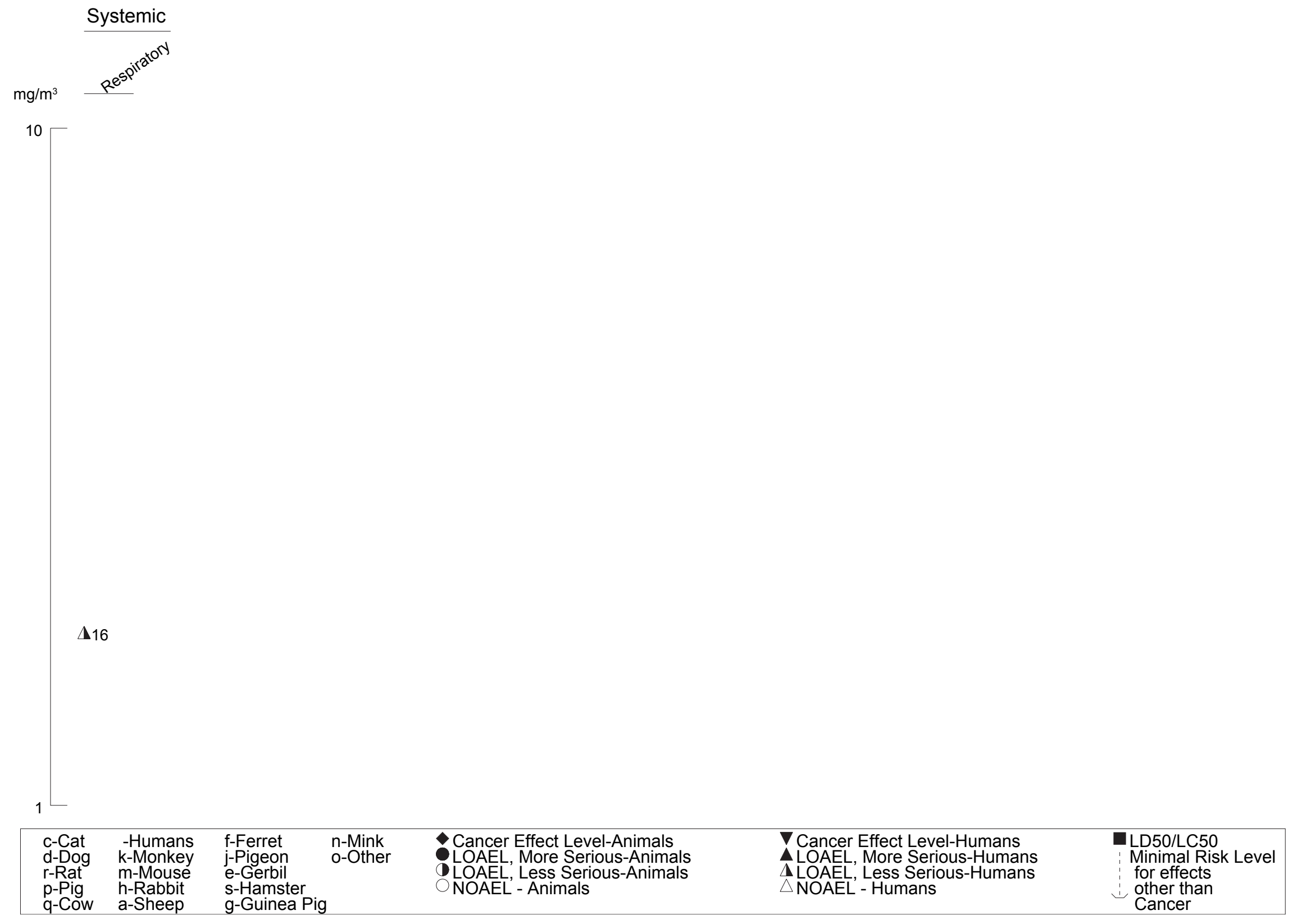

\begin{tabular}{|c|c|c|c|c|c|c|}
\hline $\begin{array}{l}\text { c-Cat } \\
\text { d-Dog } \\
\text { r-Rat } \\
\text { p-Pig } \\
\text { q-Cow }\end{array}$ & $\begin{array}{l}\text {-Humans } \\
\text { k-Monkey } \\
\text { m-Mouse } \\
\text { h-Rabbit } \\
\text { a-Sheep }\end{array}$ & $\begin{array}{l}\text { f-Ferret } \\
\text { j-Pigeon } \\
\text { e-Gerbil } \\
\text { s-Hamster } \\
\text { g-Guinea Pig }\end{array}$ & $\begin{array}{l}\text { n-Mink } \\
\text { o-Other }\end{array}$ & $\begin{array}{l}\text { Cancer Effect Level-Animals } \\
\text { QLAEL, More Serious-Animals } \\
\text { DOAEL, Less Serious-Animals } \\
\text { NOAEL - Animals }\end{array}$ & $\begin{array}{l}\text { V Cancer Effect Level-Humans } \\
\text { A LOAEL, More Serious-Humans } \\
\text { A LOAEL, Less Serious-Humans } \\
\triangle \text { NOAEL - Humans }\end{array}$ & $\begin{array}{l}\text { LD50/LC50 } \\
\text { Minimal Risk Level } \\
\text { for effects } \\
\text { other than } \\
\text { Cancer }\end{array}$ \\
\hline
\end{tabular}


respiratory irritation symptoms increased with the mean dust concentrations for different job categories in the plant, the cross-sectional design of the Garabrant et al. (1985) study prevented the determination of whether the elevation of acute respiratory symptoms was a consequence of acute or repeated exposure to sodium borate dusts. Thus, the effect levels associated with the measured prevalence of acute respiratory symptoms in this study are not included in Table 3-1 or Figure 3-1.

The occurrence of acute respiratory symptoms as a possible consequence of acute exposure to dusts of sodium borate (the decahydrate, pentahydrate, and anhydrous tetraborates) was studied in a later study by interviewing workers about acute irritation symptoms before the work shift began and at regular hourly intervals during the work shift, and by measuring personal air concentrations of particulates at concurrent intervals for 4 consecutive days (Hu et al. 1992; Wegman et al. 1991, 1994). Seventy-nine exposed production workers and 27 nonexposed workers were included in the study. In the latest analysis of the collected data, the incidence rates for irritation symptoms in exposed workers were statistically significantly higher than those in nonexposed workers, with exposed workers 9-, 5-, and 3-fold more likely to report incidents of nasal, eye, and throat irritation, respectively, than comparison workers (Hu et al. 1992; Wegman et al. 1991, 1994). For the unexposed groups, the arithmetic means of the 6-hour TWA daily dust concentration was $0.45 \mathrm{mg}$ particulates $/ \mathrm{m}^{3}\left(0.02 \mathrm{mg}\right.$ boron $\left./ \mathrm{m}^{3}\right)$, with $100 \%$ of samples $\leq 1.0 \mathrm{mg}$ particulates $/ \mathrm{m}^{3}$ and about $90 \%$ of samples $\leq 0.5 \mathrm{mg}$ particulates $/ \mathrm{m}^{3}$. The arithmetic mean of the 6-hour TWA daily dust concentrations for the exposed group was $5.72 \mathrm{mg}$ particulates $/ \mathrm{m}^{3}(0.44 \mathrm{mg}$ boron $/ \mathrm{m}^{3}$ ), with a majority of air concentrations between 1.0 and $10.0 \mathrm{mg}$ particulates $/ \mathrm{m}^{3}$ (Wegman et al. 1991, 1994). Several factors make it difficult to identify a NOAEL or LOAEL for this study, precluding its inclusion in Table and Figure 3-1. Study participants rated their perception of individual episodes of eye, throat, and nasal irritancy on a scale of 0 (not at all) to 10 (very, very much), with ratings of 1 , 2, and 3 representing “very little”, “fairly little”, and “moderate” irritation, respectively. Unexposed responders to the pre-shift survey reported a mean rating of 1.9 for all symptoms, while the mean rating for nasal irritation (the most commonly reported effect) among exposed workers was just slightly higher at 2.2. Thus, the cutoff for boron dust-induced effects is likely to be for ratings of $\geq 3$. In the exposed group, $91 \%$ of reported symptoms were rated with severity scores $\leq 3$ and $96 \%$ of symptoms were rated with severity scores of $\leq 4$ (“pretty much”). For incidences in which the workers depressed a counter button to record time of symptom onset, and the study surveyor recorded an occurrence of effect, the probability of reporting an effect did not change markedly until 0.25-hour TWA exposure levels reached 0.8-1.2 mg boron $/ \mathrm{m}^{3}$ (Wegman et al. 1991). There was no significant difference in reporting of symptoms based on the type of borate dust, which differ in boron content by almost a factor of two (i.e., $4.7 \mathrm{mg}$ anhydrous borax $/ \mathrm{m}^{3}$ and $8.8 \mathrm{mg} / \mathrm{m}^{3}$ of the decahydrate provide $1 \mathrm{mg}$ boron $/ \mathrm{m}^{3}$ ). Finally, the data do not indicate a 
temporal increase in effect intensity, as would be expected for a local irritant. Further, since no chronic effects (i.e., reduced $\mathrm{FEV}_{1}$ ) were observed in workers assessed by Wegman et al. (1994), 7 years after being assessed by Garabrant et al. (1985), the acute-duration inhalation MRL of $0.3 \mathrm{mg} / \mathrm{m}^{3}$ should also be health-protective for intermediate- and chronic-duration exposures.

Acute-duration laboratory exposures of volunteers to boric acid and sodium borate (sodium borate pentahydrate) dust provide more precise estimates of effect levels than the occupational studies. Male and female volunteers were trained to recognize the difference in chemesthetic feel (pungency or irritancy) of various levels of $\mathrm{CO}_{2}$ offered to the eyes, nose, and throat (Cain et al. 2004, 2008). Exposures of $\geq 17.7 \% \mathrm{CO}_{2}$ resulted in a "feel" described by the volunteers as irritating. Male volunteers exposed to $\leq 3.0 \mathrm{mg}$ boron $/ \mathrm{m}^{3}$ ( $\leq 20 \mathrm{mg}$ sodium borate $/ \mathrm{m}^{3}$ ) for 20 minutes reported increasingly higher magnitude of feel of the dust in eyes, nose, and throat. However, the mean reported level of feel, compared to equivalent $\mathrm{CO}_{2}$ levels, was not considered irritating by the study subjects. The mean reported perception of feel at $\geq 4.5 \mathrm{mg}$ boron $/ \mathrm{m}^{3}$ ( $\geq 30$ sodium borate $\left./ \mathrm{m}^{3}\right)$ was reported to feel irritating to the nose (Cain et al. 2004). Significantly increased nasal secretions (by mass) occurred at $1.5 \mathrm{mg}$ boron $/ \mathrm{m}^{3}$ (10 mg sodium borate $/ \mathrm{m}^{3}$ ), but not $0.8 \mathrm{mg}$ boron $/ \mathrm{m}^{3}$ (5 mg sodium borate $/ \mathrm{m}^{3}$ ) (Cain et al. 2004). In a similar experiment, male and female volunteers exposed to $1.5 \mathrm{mg}$ boron $/ \mathrm{m}^{3}$ (10 $\mathrm{mg}$ sodium borate $/ \mathrm{m}^{3}$ ) or $1.8 \mathrm{mg}$ boron $/ \mathrm{m}^{3}$ (10 $\mathrm{mg}$ boric acid $/ \mathrm{m}^{3}$ ) for 47 minutes while exercising reported a mean sense of feel that initially increased and peaked at the equivalent of slightly less than $17.7 \% \mathrm{CO}_{2}$. These boron exposures, with a mean reported feel equivalent to $<17.7 \% \mathrm{CO}_{2}$, were considered non-irritating by the study subjects, although increases in nasal secretions were observed at $1.8 \mathrm{mg}$ boron $/ \mathrm{mg}^{3}$ (10 mg boric acid $/ \mathrm{m}^{3}$ ), but not at $0.9 \mathrm{mg}$ boron $/ \mathrm{mg}^{3}$ (5 mg boric acid $/ \mathrm{m}^{3}$ ) (Cain et al. 2008). The volume of nasal secretions increased above controls for the first 20 minutes of exposure, but did not continue to increase thereafter, suggesting that increased secretions resulted from possible dust-induced changes in osmolarity rather than an irritation response. No dose-related changes in nasal airway resistance or breathing patterns were observed. Together, these studies were used to identify a minimal LOAEL of $1.5 \mathrm{mg}$ boron $/ \mathrm{m}^{3}$, with an associated NOAEL of $0.8 \mathrm{mg} / \mathrm{m}^{3}$, for significantly increased mass of nasal secretions, from which an acute-duration inhalation MRL of $0.3 \mathrm{mg}$ boron $/ \mathrm{m}^{3}$ was derived (see Appendix A).

Corroborating evidence in animals for the respiratory irritation potential of inhaled boron compounds is sparse. Mild respiratory irritation, indicated by a $20 \%$ reduction in breathing rate, occurred in mice inhaling $53 \mathrm{mg}$ boron $/ \mathrm{m}^{3}$ (300 mg boric acid $/ \mathrm{m}^{3}$ ) for 3 hours (Krystofiak and Schaper 1996). Rats were exposed to aerosols of boron oxide (6 hours/day, 5 days/week) at concentrations of $470 \mathrm{mg}$ boron oxide/ $\mathrm{m}^{3}$ (73 mg boron $/ \mathrm{m}^{3}$ ) for 10 weeks, $175 \mathrm{mg}$ boron oxide $/ \mathrm{m}^{3}$ (27 mg boron $/ \mathrm{m}^{3}$ ) for 12 weeks, or 
$77 \mathrm{mg}$ boron oxide $/ \mathrm{m}^{3}$ (12 $\mathrm{mg}$ boron $/ \mathrm{m}^{3}$ ) for 24 weeks, and dogs were exposed to $57 \mathrm{mg}$ boron oxide $/ \mathrm{m}^{3}$ (9 $\mathrm{mg}$ boron/ $\mathrm{m}^{3}$ ) for 23 weeks (Wilding et al. 1959). In rats, histological examination of a comprehensive set of tissues revealed no differences between the tissues of exposed and control animals. Signs of gross toxicity were restricted to the appearance of a reddish exudate from the nose of some of the rats exposed to $470 \mathrm{mg}$ boron oxide $/ \mathrm{m}^{3}$.

Mice (ICR) exposed to $0.7 \mathrm{ppm}$ diborane gas $\left(0.2 \mathrm{mg}\right.$ boron $\left./ \mathrm{m}^{3}\right)$ for 2 or 4 weeks exhibited slight infiltration of polymorphous neutrophil in peribronchiolar regions of the respiratory tract (Nomiyama et al. 1995). Mice exposed to $5 \mathrm{ppm}$ diborane gas $\left(1.7 \mathrm{mg}\right.$ boron $\left./ \mathrm{m}^{3}\right)$ for 2 weeks exhibited increased lung weight, nasal cavity changes, diffuse panbrochiolitis-like lesions, cellular infiltration of the bronchioles and perivascular area, appearance of alveolar macrophages, perivascular lymphoid hyperplasia, lung congestion, bleeding, and edema (Uemura et al. 1995).

Comparison of the results from the studies of mice exposed to diborane gas (Nomiyama et al. 1995; Uemura et al. 1995) and those from studies of rats and dogs exposed to boron oxide (Wilding et al. 1959) indicate that diborane gas is much more potent as a respiratory toxicant than boron oxide. Diborane gas is expected to have a very short half-life in the environment because of its reactivity. Thus, it is not expected to be a significant environmental toxicant, except in workplaces where it might be used and accidentally released.

Cardiovascular Effects. Rats exposed to aerosols of boron oxide at a concentration of $470 \mathrm{mg}$ boron oxide/ $\mathrm{m}^{3}$ (73 $\mathrm{mg}$ boron $/ \mathrm{m}^{3}$ ) for 10 weeks, $175 \mathrm{mg}$ boron oxide $/ \mathrm{m}^{3}$ (27 $\mathrm{mg}$ boron $/ \mathrm{m}^{3}$ ) for 12 weeks, or $77 \mathrm{mg}$ boron oxide $/ \mathrm{m}^{3}$ (12 mg boron $/ \mathrm{m}^{3}$ ) for 24 weeks showed no gross or microscopic effects in the cardiovascular system (Wilding et al. 1959).

Gastrointestinal Effects. No gross or microscopic changes were seen in the gastrointestinal tract of rats exposed to aerosols of boron oxide at a concentration of $470 \mathrm{mg}$ boron oxide $/ \mathrm{m}^{3}$ (73 $\mathrm{mg}$ boron $/ \mathrm{m}^{3}$ ) for 10 weeks, $175 \mathrm{mg}$ boron oxide/ $\mathrm{m}^{3}$ ( $27 \mathrm{mg}$ boron $/ \mathrm{m}^{3}$ ) for 12 weeks, or $77 \mathrm{mg}$ boron oxide $/ \mathrm{m}^{3}$ (12 mg boron $/ \mathrm{m}^{3}$ ) for 24 weeks (Wilding et al. 1959).

Hematological Effects. Rats exposed to aerosols of boron oxide at concentrations of $470 \mathrm{mg}$ boron oxide/ $\mathrm{m}^{3}$ (73 mg boron $/ \mathrm{m}^{3}$ ) for 10 weeks, $175 \mathrm{mg}$ boron oxide $/ \mathrm{m}^{3}$ (27 mg boron $/ \mathrm{m}^{3}$ ) for 12 weeks, or $77 \mathrm{mg}$ boron oxide $/ \mathrm{m}^{3}$ (12 $\mathrm{mg}$ boron $/ \mathrm{m}^{3}$ ) for 24 weeks, and dogs exposed to aerosols of boron oxide at 
concentrations of $57 \mathrm{mg}$ boron oxide $/ \mathrm{m}^{3}\left(9 \mathrm{mg}\right.$ boron $\left./ \mathrm{m}^{3}\right)$ for 23 weeks showed no significant changes in total red and white blood cell count, hemoglobin, hematocrit, or differential count (Wilding et al. 1959).

Musculoskeletal Effects. No gross or microscopic effects of exposure were observed in the femur, rib, and muscle of rats exposed to concentrations of $470 \mathrm{mg}$ boron oxide $/ \mathrm{m}^{3}\left(73 \mathrm{mg}\right.$ boron $/ \mathrm{m}^{3}$ ) for 10 weeks, $175 \mathrm{mg}$ boron oxide $/ \mathrm{m}^{3}$ (27 mg boron $/ \mathrm{m}^{3}$ ) for 12 weeks, or $77 \mathrm{mg}$ boron oxide $/ \mathrm{m}^{3}$ (12 mg boron $/ \mathrm{m}^{3}$ ) for 24 weeks (Wilding et al. 1959).

Hepatic Effects. No gross or microscopic hepatic effects were observed in rats exposed to aerosols of boron oxide at concentrations of $470 \mathrm{mg}$ boron oxide $/ \mathrm{m}^{3}$ (73 $\mathrm{mg}$ boron $/ \mathrm{m}^{3}$ ) for 10 weeks, $175 \mathrm{mg}$ boron oxide/ $\mathrm{m}^{3}$ (27 mg boron $/ \mathrm{m}^{3}$ ) for 12 weeks, or $77 \mathrm{mg}$ boron oxide $/ \mathrm{m}^{3}$ (12 $\mathrm{mg}$ boron $/ \mathrm{m}^{3}$ ) for 24 weeks. No hepatic effects were indicated from serum chemistry of dogs exposed to aerosols of boron oxide at concentrations of $57 \mathrm{mg}$ boron oxide $/ \mathrm{m}^{3}\left(9 \mathrm{mg}\right.$ boron $/ \mathrm{m}^{3}$ ) for 23 weeks (Wilding et al. 1959).

Renal Effects. No gross or microscopic renal effects were observed in rats exposed to aerosols of boron oxide at concentrations of $470 \mathrm{mg}$ boron oxide $/ \mathrm{m}^{3}$ (73 $\mathrm{mg}$ boron $/ \mathrm{m}^{3}$ ) for 10 weeks, $175 \mathrm{mg}$ boron oxide $/ \mathrm{m}^{3}$ (27 mg boron $/ \mathrm{m}^{3}$ ) for 12 weeks, or $77 \mathrm{mg}$ boron oxide $/ \mathrm{m}^{3}$ (12 $\mathrm{mg}$ boron $/ \mathrm{m}^{3}$ ) for 24 weeks. No renal effects were indicated from serum chemistry of dogs exposed to aerosols of boron oxide at concentrations of $57 \mathrm{mg}$ boron oxide/ $\mathrm{m}^{3}\left(9 \mathrm{mg}\right.$ boron $/ \mathrm{m}^{3}$ ) for 23 weeks (Wilding et al. 1959).

Ocular Effects. Human occupational exposure to a mean concentration of $0.44 \mathrm{mg}$ boron $/ \mathrm{m}^{3}$ (5.72 $\mathrm{mg}$ particulates $/ \mathrm{m}^{3}$ ) (Hu et al. 1992; Wegman et al. 1994) and 1.8-3.1 $\mathrm{mg}$ boron $/ \mathrm{m}^{3}(8.4-14.6 \mathrm{mg}$ particulates $/ \mathrm{m}^{3}$ ) (Garabrant et al. 1984, 1985) as sodium borate dust produced eye irritation following acute-duration exposures. The cross-sectional study design of Garabrant et al. $(1984,1985)$ made it difficult to determine whether the observed effects were caused by acute or repeated exposures; however, the design employed by Wegman et al. (1994), which included 6-hour TWA air samples and worker reports of irritancy before the start of the work shift and during the shift, allowed the determination that this acute ocular irritation was due to acute exposure. No ocular effects were observed in rats exposed to aerosols of boron oxide at concentrations of $470 \mathrm{mg}$ boron oxide $/ \mathrm{m}^{3}\left(73 \mathrm{mg}\right.$ boron $/ \mathrm{m}^{3}$ ) for 10 weeks, $175 \mathrm{mg}$ boron oxide $/ \mathrm{m}^{3}$ (27 mg boron $/ \mathrm{m}^{3}$ ) for 12 weeks, or $77 \mathrm{mg}$ boron oxide/ $\mathrm{m}^{3}$ (12 $\mathrm{mg}$ boron $/ \mathrm{m}^{3}$ ) for 24 weeks (Wilding et al. 1959). 


\subsubsection{Immunological and Lymphoreticular Effects}

No gross or microscopic effects on immunological or lymphoreticular tissues (lymph nodes and spleens) were observed in rats exposed to aerosols of boron oxide at concentrations of $470 \mathrm{mg}$ boron oxide $/ \mathrm{m}^{3}$ (73 mg boron $/ \mathrm{m}^{3}$ ) for 10 weeks, $175 \mathrm{mg}$ boron oxide $/ \mathrm{m}^{3}$ ( $27 \mathrm{mg}$ boron $/ \mathrm{m}^{3}$ ) for 12 weeks, or $77 \mathrm{mg}$ boron oxide/ $\mathrm{m}^{3}$ (12 mg boron $/ \mathrm{m}^{3}$ ) for 24 weeks (Wilding et al. 1959).

\subsubsection{Neurological Effects}

No gross or microscopic effects on the brain were observed in rats exposed to aerosols of boron oxide at concentrations of $470 \mathrm{mg}$ boron oxide $/ \mathrm{m}^{3}$ (73 mg boron $/ \mathrm{m}^{3}$ ) for 10 weeks, $175 \mathrm{mg}$ boron oxide/ $\mathrm{m}^{3}$ (27 mg boron $/ \mathrm{m}^{3}$ ) for 12 weeks, or $77 \mathrm{mg}$ boron oxide $/ \mathrm{m}^{3}$ (12 $\mathrm{mg}$ boron $/ \mathrm{m}^{3}$ ) for 24 weeks (Wilding et al. 1959).

\subsubsection{Reproductive Effects}

A study of 28 male boric acid production workers occupationally exposed to $22-80 \mathrm{mg} / \mathrm{m}^{3}$ boron aerosols (boron form uncertain) for $\geq 10$ years (Tarasenko et al. 1972) revealed low sperm counts, reduced sperm motility, and elevated fructose content of seminal fluids, compared to controls. These effects are consistent with high-dose animal exposures. However, this study is limited by the small number of subjects and limited data reporting. Furthermore, a cross-sectional survey of 753 employees working for at least 9 months at a borax production facility in California found worker fertility rates to be higher than the U.S. national average (Whorton et al. 1994). However, this study is limited by lack of exposure data.

In animals, no gross or microscopic effects were found on the ovary or testes of rats exposed to aerosols of boron oxide at concentrations of $470 \mathrm{mg}$ boron oxide/ $\mathrm{m}^{3}$ (73 mg boron $/ \mathrm{m}^{3}$ ) for 10 weeks, $175 \mathrm{mg}$ boron oxide $/ \mathrm{m}^{3}$ ( $27 \mathrm{mg}$ boron $/ \mathrm{m}^{3}$ ) for 12 weeks, or $77 \mathrm{mg}$ boron oxide $/ \mathrm{m}^{3}$ (12 $\mathrm{mg}$ boron $/ \mathrm{m}^{3}$ ) for 24 weeks (Wilding et al. 1959).

\subsubsection{Developmental Effects}

No studies were located regarding developmental effects in humans or animals after inhalation exposure to boron.

\subsubsection{Cancer}

No studies were located regarding cancer in humans or animals after inhalation exposure to boron. 


\subsubsection{Oral Exposure}

Data for boron oral toxicity in humans involves exposure to the borates or boric acid. These boroncontaining compounds are primarily found in food and water and have been implicated in numerous accidental or intentional poisonings in human case reports. Similarly, the boron toxicity studies in animals have utilized exposures to borates or boric acid.

\subsubsection{Death}

Case reports of lethal oral exposure of humans to boron primarily involve accidental or intentional exposures to high levels of boric acid. Similar clinical signs have been seen in adults and children. A review of 784 primarily acute boric acid exposures in adults and children found that $88 \%$ of cases were asymptomatic. The reports did not contain information on the dose response for boric acid, as symptomatic cases had doses ranging from $100 \mathrm{mg}$ to $55 \mathrm{~g}$ boric acid (18 mg-10 g boron), while asymptomatic cases had doses ranging from $10 \mathrm{mg}$ to $89 \mathrm{~g}$ boric acid ( $2 \mathrm{mg}-16 \mathrm{~g}$ boron) (Litovitz et al. 1988). Nonetheless, death occurred in some children following oral doses in this wide dose range. Five infants who ingested formula accidentally prepared with $2.5 \%$ aqueous solution of boric acid became lethargic, developed vomiting and diarrhea, and died within 3 days after exposure (Wong et al. 1964). The estimated boric acid consumption ranged from 4.51 to $14 \mathrm{~g}$ (0.8-2.5g boron). In two infants who ingested $9.25 \mathrm{~g}$ boric acid (505 mg boron/kg/day) and $14 \mathrm{~g}$ boric acid (765 mg boron/kg/day), degenerative changes were seen in the liver, kidney, and brain (Wong et al. 1964). In a food poisoning incident in Malaysia, 13 children died after consuming Chinese noodles contaminated with boric acid (Chao et al. 1991a, 1991b). The deaths were determined by the study authors to be caused by unknown levels of aflatoxin and boric acid in the noodles. Clinical signs included vomiting, pyrexia, diarrhea, abdominal pain, anorexia, giddiness, seizures, and eventual coma. Postmortem examination revealed coagulative necrosis of the liver, proliferative metaplasia of the hepatocytes, giant cell formation, central vein sclerosis, bile stasis, and steatosis, acute renal tubule necrosis, upper gastrointestinal erosion, and encephalopathy. However, the relative contribution of boric acid to these effects could not be determined.

A common suite of symptoms was presented in case reports of adult oral exposures that resulted in death. A 77-year-old man ingested a single dose of $30 \mathrm{~g}$ of boric acid (85 mg boron/kg) to cure hiccups (Ishii et al. 1993). Effects included vomiting, diarrhea, erythema, cyanotic extremities, acute renal failure, cardiopulmonary hypotension, and death from cardiac insufficiency. Almost identical clinical signs and 
death occurred in a 45-year-old man ingesting approximately $49 \mathrm{~g}$ of boron (280 g boric acid, $700 \mathrm{mg}$ boron/kg assuming a body weight of $70 \mathrm{~kg}$ ) (Restuccio et al. 1992).

In animals, rats appear to be more sensitive than dogs or mice to the lethal effects of acute boron exposures. Oral $\mathrm{LD}_{50}$ values for respective boron equivalents of boric acid or borax were 898 and $642 \mathrm{mg} / \mathrm{kg}$ in an unspecified rat strain (Smyth et al. 1969), 600 and $510 \mathrm{mg} / \mathrm{kg}$ in Sprague-Dawley rats (Weir and Fisher 1972), and 550 and 690 mg/kg in Long Evans rats (Weir and Fisher 1972). No deaths were reported in dogs exposed to a single dose of $696 \mathrm{mg}$ boron $/ \mathrm{kg}$ (3,977 $\mathrm{mg}$ boric acid/kg) and $738 \mathrm{mg}$ boron/kg (5,549 mg borax/kg) (Weir and Fisher 1972). No single-dose $\mathrm{LD}_{50}$ studies in mice were available; however, mortality rates of 20 and $60 \%$ were observed in males given 14 daily doses of 2,251 and 3,671 $\mathrm{mg}$ boron/ $\mathrm{kg} /$ day (12.9 or $21.0 \mathrm{~g}$ boric acid/kg/day) in the diet, respectively (NTP 1987), but not at $926 \mathrm{mg}$ boron/kg/day (5.3 g boric acid/kg/day). These animals were lethargic and exhibited discolored spleen, liver, and renal medullae and hyperplasia and dysplasia of the forestomach (NTP 1987).

With intermediate-duration oral exposure, rats appear to be slightly more sensitive than mice to the lethality of boric acid. There was 100\% mortality in Sprague-Dawley rats fed $450 \mathrm{mg}$ boron/kg/day within a 6-week period (Weir and Fisher 1972). Congestion of liver and kidneys, small gonads, and brain swelling were reported. Eighty percent of male and 60\% of female B6C3F1 mice died in a study involving exposure to $577 \mathrm{mg}$ boron/kg/day (3,298 $\mathrm{mg}$ boric acid/kg/day) in the diet for up to 90 days (NTP 1987). Hyperkeratosis and/or acanthosis in the stomach and extramedullary hematopoiesis of the spleen in both sexes of mice were observed at the same dose level.

With chronic exposure to boric acid in the diet, mortality at 103 weeks was $\geq 40$ and $\geq 30 \%$ in male and female B6C3F1 mice, respectively, exposed to $\geq 79 \mathrm{mg}$ boron/ $\mathrm{kg} /$ day ( $\geq 450 \mathrm{mg}$ boric acid $/ \mathrm{kg} /$ day), compared to 18 and 34\% in untreated male and female controls, respectively (NTP 1987). No clinical signs were reported for either sex; however, boron caused an increased incidence of testicular atrophy and interstitial hyperplasia in male mice exposed to doses $\geq 201 \mathrm{mg}$ boron/kg/day (1,150 $\mathrm{mg}$ boric acid/kg/day). 
$\mathrm{LD}_{50}$ values and, in some cases, the lowest levels at which death was reported in humans and animals and the duration categories are recorded in Table 3-2 and plotted in Figure 3-2.

\subsubsection{Systemic Effects}

No studies were located regarding respiratory effects in animals or musculoskeletal effects in humans or animals after oral exposure to boron.

Information on respiratory, gastrointestinal, hematological, cardiovascular, hepatic, renal, and dermal/ocular effects is discussed below. The highest NOAEL values and all reliable LOAEL values for these systemic effects for each species and duration category are recorded in Table 3-2 and plotted in Figure 3-2.

Respiratory Effects. Widespread vascular congestion and hemorrhages in the lungs were reported in one infant who ingested an estimated dose of $505 \mathrm{mg}$ boron/kg/day for 3-5 days (Wong et al. 1964).

Cardiovascular Effects. Ingestion of $85 \mathrm{mg}$ boron/kg (as $30 \mathrm{~g}$ of boric acid) by a 77-year-old man (Ishii et al. 1993) resulted in cardiopulmonary hypotension and death from cardiac insufficiency.

Gastrointestinal Effects. Ingestion of boron in humans can cause gastrointestinal effects. Nausea, persistent vomiting, diarrhea, and colicky abdominal pain in infants were associated with acute ingestion of a total of $\geq 184 \mathrm{mg}$ boron/kg/day (based on $1.9 \mathrm{~kg}$ body weight) as boric acid, which was accidentally incorporated in infant formula (Wong et al. 1964). Vomiting and diarrhea occurred following ingestion of $85 \mathrm{mg}$ boron $/ \mathrm{kg}$ (as 30g of boric acid) by a 77-year-old man (Ishii et al. 1993). Vomiting was the only sign of boron toxicity in two adult females who ingested $14 \mathrm{~g}$ boron ( $80 \mathrm{~g}$ boric acid) in a fungicide and $52 \mathrm{~g}$ boron (297 $\mathrm{g}$ boric acid) in a suicide attempt. In the absence of body weight data, doses for these cases could not be estimated. The subjects were hospitalized for 24-96 hours and did not develop further symptoms following release (Linden et al. 1986). Food poisoning of 13 children with unknown levels of aflatoxin and boric acid (Chao et al. 1991a, 1991b) resulted in vomiting, diarrhea, abdominal pain, anorexia, and upper gastrointestinal erosion. 
Table 3-2 Levels of Significant Exposure to Boron - Oral

\begin{tabular}{|c|c|c|c|c|c|c|c|c|c|}
\hline \multirow[b]{2}{*}{$\begin{array}{l}\text { Key to } \\
\text { Figure } \\
\end{array}$} & \multirow[b]{2}{*}{$\begin{array}{l}\text { Species } \\
\text { (Strain) }\end{array}$} & \multirow{2}{*}{$\begin{array}{l}\text { Exposurel } \\
\text { Durationl } \\
\text { Frequency } \\
\text { (Route) }\end{array}$} & \multicolumn{6}{|c|}{ LOAEL } & \multirow[b]{2}{*}{ Comments } \\
\hline & & & System & $\begin{array}{l}\text { NOAEL } \\
\text { (mg/kg/day) }\end{array}$ & \multirow[t]{2}{*}{$\begin{array}{r}\text { Less Serious } \\
(\mathrm{mg} / \mathrm{kg} / \mathrm{day}) \\
\end{array}$} & \multicolumn{2}{|c|}{$\begin{array}{l}\text { Serious } \\
\text { (mg/kg/day) }\end{array}$} & $\begin{array}{l}\text { Reference } \\
\text { Chemical Form } \\
\end{array}$ & \\
\hline \multicolumn{9}{|c|}{$\begin{array}{l}\text { ACUTE EXPOSURE } \\
\text { Death }\end{array}$} & \\
\hline 1 & $\begin{array}{l}\text { Rat } \\
\text { (NS) }\end{array}$ & $1 \times / d$ & & & & 642 & (LD50) & $\begin{array}{l}\text { Smyth et al. } 1969 \\
\text { Borax }\end{array}$ & \\
\hline 2 & $\begin{array}{l}\text { Rat } \\
\text { (NS) }\end{array}$ & $1 \times / d$ & & & & 898 & (LD50) & $\begin{array}{l}\text { Smyth et al. } 1969 \\
\text { Boric acid }\end{array}$ & \\
\hline 3 & $\begin{array}{l}\text { Rat } \\
\text { (Sprague- } \\
\text { Dawley) }\end{array}$ & $\begin{array}{l}1 d \\
(G)\end{array}$ & & & & 600 & (LD50) & $\begin{array}{l}\text { Weir and Fisher } 1972 \\
\text { Boric acid }\end{array}$ & \\
\hline 4 & $\begin{array}{l}\text { Rat } \\
\text { (Long- Eva }\end{array}$ & $\begin{array}{r}1 d \\
n s)(G)\end{array}$ & & & & 550 & (LD50) & $\begin{array}{l}\text { Weir and Fisher } 1972 \\
\text { Boric acid }\end{array}$ & \\
\hline 5 & $\begin{array}{l}\text { Rat } \\
\text { (Sprague- } \\
\text { Dawley) }\end{array}$ & $\begin{array}{l}1 d \\
(G)\end{array}$ & & & & 510 & (LD50) & $\begin{array}{l}\text { Weir and Fisher } 1972 \\
\text { Borax }\end{array}$ & \\
\hline 6 & $\begin{array}{l}\text { Rat } \\
\text { (Long- Eva }\end{array}$ & $\begin{array}{r}1 d \\
n s)(G)\end{array}$ & & & & 690 & (LD50) & $\begin{array}{l}\text { Weir and Fisher } 1972 \\
\text { Borax }\end{array}$ & \\
\hline 7 & $\begin{array}{l}\text { Mouse } \\
\text { (B6C3F1) }\end{array}$ & $\begin{array}{l}14 \mathrm{~d} \\
(\mathrm{~F})\end{array}$ & & & & 2251 & (20\% mortality) & $\begin{array}{l}\text { NTP 1987, Dieter } 1994 \\
\text { Boric acid }\end{array}$ & $\begin{array}{l}\text { Higher percentage } \\
\text { mortality at higher } \\
\text { exposure levels. }\end{array}$ \\
\hline
\end{tabular}


Table 3-2 Levels of Significant Exposure to Boron - Oral

\begin{tabular}{|c|c|c|c|c|c|}
\hline $\begin{array}{l}\text { Key to } \\
\text { Figure } \\
\end{array}$ & $\begin{array}{c}\text { Species } \\
\text { (Strain) }\end{array}$ & $\begin{array}{l}\text { Exposurel } \\
\text { Durationl } \\
\text { Frequency } \\
\text { (Route) }\end{array}$ & System & $\begin{array}{l}\text { NOAEL } \\
\text { (mg/kg/day) }\end{array}$ & $\begin{array}{r}\text { Less Serious } \\
\text { (mg/kg/day) }\end{array}$ \\
\hline \multicolumn{6}{|c|}{ Systemic } \\
\hline 8 & Human & $\begin{array}{l}3-5 d \\
(F)\end{array}$ & Resp & & \\
\hline & & & Gastro & & 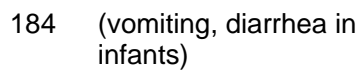 \\
\hline
\end{tabular}

LOAEL

$\begin{array}{ll}\text { Serious } & \text { Reference } \\ \text { (mg/kg/day) } & \text { Chemical Form }\end{array}$

Comments

Hepatic

505 (parenchymatous

degeneration, jaundice

fatty changes, congestion

in one infant who died)

Renal

765 (parenchymatous

degeneration, reduced
urine output, protein in

urine in one infant who

died)

Dermal

505 (erythema,

desquamation, extensive

shedding of skin in one

infant who died)

11 infants; renal,

hepatic, and

respiratory effects from

post-mortem

observations of 2 fatal

cases.

Gastro

926

2251 (gastric hyperplasia and dysplasia)

NTP 1987, Dieter 1994 Boric acid 
Table 3-2 Levels of Significant Exposure to Boron - Oral

\begin{tabular}{|c|c|c|c|c|c|}
\hline $\begin{array}{l}\text { Key to } \\
\text { Figure }\end{array}$ & $\begin{array}{l}\text { Species } \\
\text { (Strain) }\end{array}$ & $\begin{array}{l}\text { Exposurel } \\
\text { Durationl } \\
\text { Frequency } \\
\text { (Route) }\end{array}$ & System & $\begin{array}{l}\text { NOAEL } \\
\text { (mg/kg/day) }\end{array}$ & $\begin{array}{r}\text { Less Serious } \\
(\mathrm{mg} / \mathrm{kg} / \mathrm{day})\end{array}$ \\
\hline 10 & $\begin{array}{l}\text { Dog } \\
\text { (Mongrel) }\end{array}$ & $\begin{array}{l}1 \mathrm{~d} \\
(\mathrm{G})\end{array}$ & Gastro & & 1000 \\
\hline
\end{tabular}

LOAEL

\section{Neurological}

11 Human 3-5 d

12

Mouse $\quad 14 \mathrm{~d}$

(B6C3F1)

(F)

\section{Reproductive}

13 Rat

(Wistar) $2 \mathrm{wk}$

(G)

$14 \begin{array}{ll}\text { Rat } & 1 \times / d \\ \text { (Wistar) } & 2 \mathrm{wk}\end{array}$

(G)
3671

$53 \mathrm{M}$

$88 \mathrm{M} \mathrm{(12}$ and $13 \%$ reduction in absolute and relative

testes weight; increase in

residual body-like

structures in the testes;

increase in cellular debris

in the epididymal ducts;

exfoliation of round

spermatides, retention of

step 19 spermatids)

$44 \mathrm{M}$

$88 \mathrm{M}$ (decreased stage XII

spermatids and

increased stage $X$

pacytene spermatocytes)
Reference

Chemical Form

Comments

(mg/kg/day)

Weir and Fisher 1972

Boric acid

505

$\begin{array}{ll}\text { (perivascular } & \text { Wong et al. } 1964 \\ \text { hemorrhage, congestion, } & \text { Boric acid } \\ \text { thrombosis, and edema } & \\ \text { in brain of one infant) } & \end{array}$

in brain of one infant)

NTP 1987, Dieter 1994

Boric acid

Fukuda et al. 2000

Boric acid

Kudo et al. 2000

Boric acid
Based on 1 fatal case report.

NOAEL is for brain histopathology.

Endpoints: male

reproductive organ

veights and

histopathology and

sperm morphology.

Endpoints: male

reproductive organ

weights,

histopathology, and sperm morphology. 
Table 3-2 Levels of Significant Exposure to Boron - Oral

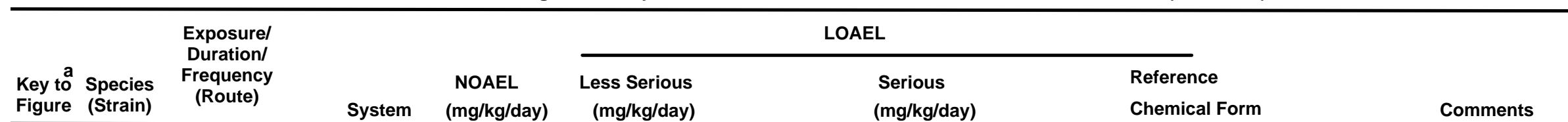

\section{Developmental}

15 Mouse

$\begin{array}{ll}\text { (CD-1) } & 2 \times / d \\ & \text { Gd } 8\end{array}$

(G)

16 Mouse $1 \mathrm{x} / \mathrm{d}$

(CD-1) $\quad$ Gd 8

(G)

17 Mouse $1 \mathrm{x} / \mathrm{d}$

(CD-1) Gd 6-10

(G)

18 Mouse $2 \times / d$
(CD-1)
Gd 6-8
(G)

19 Mouse

$\begin{array}{ll}\text { Mouse } & \text { Gd 6, 7, 9, or } \\ \text { (CD-1) } & 10\end{array}$

(G)
$70 \mathrm{~F}$ (reduced fetal weight and Cherrington and Chernoff 2002 increased skeletal

effects-cervical rib, rib

agenesis, reduced rib

length, and fused ribs)

Boric acid

$131 \mathrm{~F}$ (reduced fetal weight and Cherrington and Chernoff 2002 increased cervical Boric acid ossification)

$70 \mathrm{~F}$ (reduced fetal weight and Cherrington and Chernoff 2002 increased skeletal Boric acid abnormalities)

Cherrington and Chernoff 2002 Boric acid

\begin{tabular}{|c|c|}
\hline $\begin{array}{l}\text { F (increased skeletal } \\
\text { abnormalities) }\end{array}$ & $\begin{array}{l}\text { Cherrington and Chernoff } 2002 \\
\text { Boric acid }\end{array}$ \\
\hline $\begin{array}{l}\text { F (reduced fetal weight and } \\
\text { increased skeletal } \\
\text { abnormalities) }\end{array}$ & $\begin{array}{l}\text { Cherrington and Chernoff } 2002 \\
\text { Boric acid }\end{array}$ \\
\hline
\end{tabular}

$70 \mathrm{~F}$ (reduced fetal weight)

Cherrington and Chernoff 2002 No skeletal

Boric acid abnormalities 
Table 3-2 Levels of Significant Exposure to Boron - Oral

(continued)

\begin{tabular}{|c|c|c|}
\hline $\begin{array}{l}\text { Key to } \\
\text { Figure }\end{array}$ & $\begin{array}{l}\text { Species } \\
\text { (Strain) }\end{array}$ & $\begin{array}{l}\text { Exposu } \\
\text { Duratic } \\
\text { Frequer } \\
\text { (Rout }\end{array}$ \\
\hline 20 & $\begin{array}{l}\text { Mouse } \\
(C D-1)\end{array}$ & $\begin{array}{l}2 \times / d \\
\text { Gd } 8 \\
\text { (G) }\end{array}$ \\
\hline
\end{tabular}

21

$\begin{array}{ll}\text { Mouse } & 1 \times / d \\ \text { (CD-1) } & \text { Gd 8-14 }\end{array}$

(G)

22

$\begin{array}{ll}\text { Rabbit } & 1 \times / d \\ \text { (New } & \text { Gd 6-19 } \\ \text { Zealand) } & \text { (G) }\end{array}$

$70 \mathrm{~F}$

$22 \mathrm{~F}$
LOAEL

System (mg/kg/day) (mg/kg/day)

(mg/kg/day)
Reference

Chemical Form
$131 \mathrm{~F}$ (reduced fetal weight and Cherrington and Chernoff 2002 increased multiple skeletal malformations) Boric acid 
Table 3-2 Levels of Significant Exposure to Boron - Oral

(continued)

\begin{tabular}{|c|c|c|c|c|c|c|c|c|}
\hline \multirow[b]{2}{*}{$\begin{array}{l}\text { Key to } \\
\text { Figure }\end{array}$} & \multirow[b]{2}{*}{$\begin{array}{l}\text { Species } \\
\text { (Strain) }\end{array}$} & \multirow{2}{*}{$\begin{array}{l}\text { Exposurel } \\
\text { Durationl } \\
\text { Frequency } \\
\text { (Route) }\end{array}$} & \multicolumn{6}{|c|}{ LOAEL } \\
\hline & & & System & $\begin{array}{l}\text { NOAEL } \\
\text { (mg/kg/day) }\end{array}$ & $\begin{array}{r}\text { Less Serious } \\
\text { (mg/kg/day) }\end{array}$ & $\begin{array}{l}\text { Se } \\
\text { (mg }\end{array}$ & $\begin{array}{l}\text { ous } \\
\text { kg/day) }\end{array}$ & $\begin{array}{l}\text { Reference } \\
\text { Chemical Form }\end{array}$ \\
\hline 25 & $\begin{array}{l}\text { Mouse } \\
\text { (B6C3F1) }\end{array}$ & $\begin{array}{l}13 \text { wk } \\
(F)\end{array}$ & & & & 577 & $\begin{array}{l}\text { ( } 80 \% \text { males and } 60 \% \\
\text { females died) }\end{array}$ & $\begin{array}{l}\text { NTP 1987, Dieter } 1994 \\
\text { Boric acid }\end{array}$ \\
\hline
\end{tabular}

\section{Systemic}

26

$\begin{array}{llll}\text { Human } & 1 \mathrm{~d} / \mathrm{wk} & \text { Endocr } & 0.03 \mathrm{M}\end{array}$

Ferrando and Green 1993

Boron

NOAEL is for changes

(C)

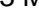

27 Rat

$30 d$

(Sprague-

(F)

Dawley)

Hepatic

$43 \mathrm{M}$

$86 \mathrm{M}(11 \%$ reduction in liver weight)

Bd Wt $\quad 172 \mathrm{M}$

28
Rat
9 wk

(Fischer- 344) (F)

29 Rat 4 wk

(Fischer- 344) (F)
Endocr
$\mathrm{Bd} \mathrm{Wt}$

$52 \mathrm{M}$

$68 \mathrm{M}(16 \%$ decrease in body weight gain)

61 M (approximately 60-78\% decrease in plasma testosterone)
Dixon et al. 1979

Borax

Ku et al. 1993a

Boric acid

Treinen and Chapin 1991

Boric acid 
Table 3-2 Levels of Significant Exposure to Boron - Oral

(continued)

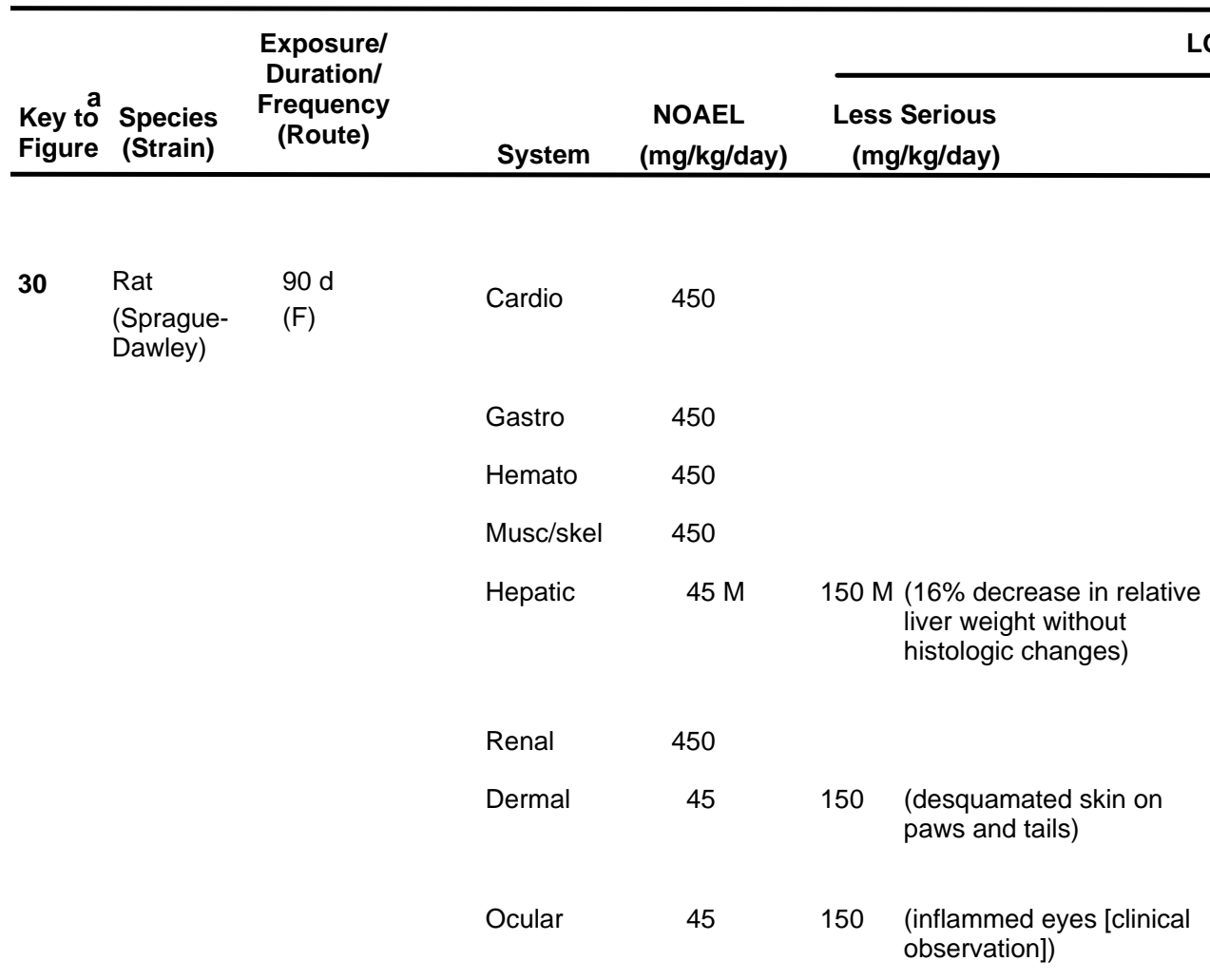

LOAEL

\section{Serious}

(mg/kg/day)

Reference

Chemical Form

Comments

Weir and Fisher 1972

Borax

NOAELS are for

histopathology and

hematology. 
Table 3-2 Levels of Significant Exposure to Boron - Oral

(continued)

\begin{tabular}{|c|c|c|c|c|c|c|}
\hline $\begin{array}{l}\text { Key to } \\
\text { Figure }\end{array}$ & $\begin{array}{l}\text { Species } \\
\text { (Strain) }\end{array}$ & $\begin{array}{l}\text { Exposurel } \\
\text { Durationl } \\
\text { Frequency } \\
\text { (Route) }\end{array}$ & System & $\begin{array}{l}\text { NOAEL } \\
(\mathrm{mg} / \mathrm{kg} / \text { day })\end{array}$ & $\begin{array}{r}\text { Les } \\
(\mathrm{m}\end{array}$ & $\begin{array}{l}\text { s Serious } \\
\text { g/kg/day) }\end{array}$ \\
\hline \multirow[t]{9}{*}{31} & $\begin{array}{l}\text { Rat } \\
\text { (Sprague- } \\
\text { Dawley) }\end{array}$ & $\begin{array}{l}90 \mathrm{~d} \\
(\mathrm{~F})\end{array}$ & Cardio & 450 & & \\
\hline & & & Gastro & 450 & & \\
\hline & & & Hemato & 450 & & \\
\hline & & & Musc/skel & 450 & & \\
\hline & & & Hepatic & $45 \mathrm{M}$ & 150 & $\begin{array}{l}\text { ( } 22 \% \text { reduction in liver } \\
\text { weight without histologic } \\
\text { changes) }\end{array}$ \\
\hline & & & Renal & 450 & & \\
\hline & & & Endocr & 450 & & \\
\hline & & & Dermal & 45 & 150 & $\begin{array}{l}\text { (desquamated skin on } \\
\text { paws and tail) }\end{array}$ \\
\hline & & & Ocular & 45 & 150 & $\begin{array}{l}\text { (inflammed eyes [clinical } \\
\text { observations]) }\end{array}$ \\
\hline
\end{tabular}

LOAEL

Serious

(mg/kg/day)

Reference

Chemical Form

Comments

Weir and Fisher $1972 \quad$ NOAELs are for

Boric acid

histopathology. 
Table 3-2 Levels of Significant Exposure to Boron - Oral

(continued)

\begin{tabular}{|c|c|c|c|c|c|c|c|c|}
\hline \multirow[b]{2}{*}{$\begin{array}{l}\text { Key to } \\
\text { Figure }\end{array}$} & \multirow[b]{2}{*}{$\begin{array}{l}\text { Species } \\
\text { (Strain) }\end{array}$} & \multirow{2}{*}{$\begin{array}{l}\text { Exposurel } \\
\text { Durationl } \\
\text { Frequency } \\
\text { (Route) }\end{array}$} & \multirow[b]{2}{*}{ System } & \multirow[b]{2}{*}{$\begin{array}{l}\text { NOAEL } \\
\text { (mg/kg/day) }\end{array}$} & \multicolumn{2}{|c|}{ LOAEL } & \multirow[b]{2}{*}{$\begin{array}{l}\text { Reference } \\
\text { Chemical Form }\end{array}$} & \multirow[b]{2}{*}{ Comments } \\
\hline & & & & & $\begin{array}{r}\text { Less Serious } \\
(\mathrm{mg} / \mathrm{kg} / \mathrm{day})\end{array}$ & $\begin{array}{l}\text { Serious } \\
\text { (mg/kg/day) }\end{array}$ & & \\
\hline
\end{tabular}

$32 \quad$ Mouse 13 wk

(B6C3F1) (F)

$\begin{array}{llll}\begin{array}{l}\text { 13 wk } \\ \text { (F) }\end{array} & 577 & \\ \text { Resp } & 577 & \\ \text { Gardio } & 288 \mathrm{M} & \begin{array}{c}577 \mathrm{M} \text { (hyperkeratosis and/or } \\ \text { acanthosis of the } \\ \text { stomach) }\end{array} \\ \text { Hemato } & 35 \mathrm{M} & \begin{array}{c}72 \mathrm{M} \text { (splenic extramedullary } \\ \text { hematopoiesis) }\end{array} \\ & & \\ \text { Hepatic } & 577 & \\ \text { Renal } & 577 & \\ \text { Endocr } & 577 & \\ \text { Dermal } & 577 & \text { 288 M (17\% reduction in body } \\ \text { Bd Wt } & 144 \mathrm{M} & \text { weight) }\end{array}$

NTP 1987, Dieter 1994

NOAELs are for (B6C3F1)

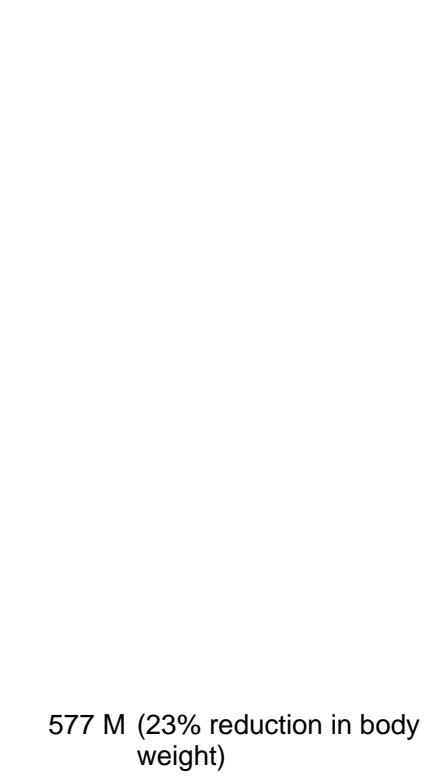


Table 3-2 Levels of Significant Exposure to Boron - Oral

(continued)

\begin{tabular}{|c|c|c|c|c|c|c|c|c|}
\hline \multirow[b]{2}{*}{$\begin{array}{l}\text { Key to } \\
\text { Figure }\end{array}$} & \multirow[b]{2}{*}{$\begin{array}{c}\text { Species } \\
\text { (Strain) }\end{array}$} & \multirow{2}{*}{$\begin{array}{l}\text { Exposurel } \\
\text { Duration/ } \\
\text { Frequency } \\
\text { (Route) }\end{array}$} & \multirow[b]{2}{*}{ System } & \multirow[b]{2}{*}{$\begin{array}{l}\text { NOAEL } \\
\text { (mg/kg/day) }\end{array}$} & \multicolumn{2}{|c|}{ LOAEL } & \multirow[b]{2}{*}{$\begin{array}{l}\text { Reference } \\
\text { Chemical Form }\end{array}$} & \multirow[b]{2}{*}{ Comments } \\
\hline & & & & & $\begin{array}{r}\text { Less Serious } \\
\text { (mg/kg/day) }\end{array}$ & $\begin{array}{l}\text { Serious } \\
\text { (mg/kg/day) }\end{array}$ & & \\
\hline
\end{tabular}

33 Dog $90 \mathrm{~d} \quad$ Cardio $\quad 60.5$

$\begin{array}{llll}\text { (Beagle) } & \text { (F) } & \text { Cardio } & 60.5\end{array}$

5

$\begin{array}{crcl}\text { Gastro } & 60.5 & & \\ \text { Hemato } & 6 & 60.5 & \begin{array}{l}\text { (decreased packed cell } \\ \text { volume and hemoglobin } \\ \text { values) }\end{array}\end{array}$

$\begin{array}{ll}\text { Musc/skel } & 60.5 \\ \text { Hepatic } & 60.5 \\ \text { Renal } & 60.5 \\ \text { Endocr } & 60.5 \\ \text { Dermal } & 60.5 \\ \text { Ocular } & 60.5\end{array}$

$\begin{array}{ll}\text { Weir and Fisher } 1972 & \begin{array}{l}\text { NOAELs are for } \\ \text { histopathology an }\end{array}\end{array}$

$\begin{array}{ll}\text { Borax } & \text { histopathology } \\ \text { hematology. }\end{array}$


Table 3-2 Levels of Significant Exposure to Boron - Oral

(continued)

\begin{tabular}{|c|c|c|c|c|c|c|c|c|}
\hline \multirow[b]{2}{*}{$\begin{array}{l}\text { Key to } \\
\text { Figure }\end{array}$} & \multirow[b]{2}{*}{$\begin{array}{c}\text { Species } \\
\text { (Strain) }\end{array}$} & \multirow{2}{*}{$\begin{array}{l}\text { Exposurel } \\
\text { Duration/ } \\
\text { Frequency } \\
\text { (Route) }\end{array}$} & \multirow[b]{2}{*}{ System } & \multirow[b]{2}{*}{$\begin{array}{l}\text { NOAEL } \\
\text { (mg/kg/day) }\end{array}$} & \multicolumn{2}{|c|}{ LOAEL } & \multirow[b]{2}{*}{$\begin{array}{l}\text { Reference } \\
\text { Chemical Form }\end{array}$} & \multirow[b]{2}{*}{ Comments } \\
\hline & & & & & $\begin{array}{r}\text { Less Serious } \\
\text { (mg/kg/day) }\end{array}$ & $\begin{array}{l}\text { Serious } \\
\text { (mg/kg/day) }\end{array}$ & & \\
\hline
\end{tabular}

34 Dog $90 d$

(Beagle) (F)

(F)

Resp

60.5

Cardio

60.5

Gastro

60.5

Hemato

60.5

Musc/skel

60.5

Hepatic

$6 \mathrm{~F}$

$60.5 \mathrm{~F}(46 \%$ increase in relative

liver weight with no

adverse histologic

changes)

$\begin{array}{ll}\text { Renal } & 60.5 \\ \text { Endocr } & 60.5 \\ \text { Dermal } & 60.5 \\ \text { Ocular } & 60.5\end{array}$

Neurological

35 Rat

Dawley)

(F)

36 Rat

Dawley)

Mouse

(B6C3F1)
13 wk

$90 \mathrm{~d}$

(F)
Weir and Fisher 1972

Boric acid

NOAELS are for

histopathology and

hematology.
Weir and Fisher 1972

Borax

Weir and Fisher 1972

Boric acid

NTP 1987, Dieter 1994

Boric acid
NOAEL is for brain histopathology. 
Table 3-2 Levels of Significant Exposure to Boron - Oral

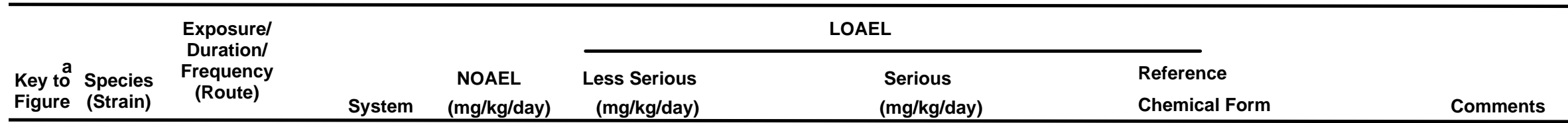

$38 \quad$ Dog $90 d$

(Beagle) (F)

\section{Reproductive}

39 Rat

(Sprague-

Dawley)

40 Rat

Dawley)

$41 \quad$ Rat

(Sprague-

Dawley)
$60.5 \mathrm{~F}$

enzyme activities;

increased plasma FSH)

$86 \mathrm{M}$ (infertility for 3 weeks

$37 \%$ decrease in

epidiymis weight;

increased plasma FSH

reduced diameter of

seminiferous tubules) following exposure)

Borax

Dixon et al. 1979

Borax

Weir and Fisher 1972

Borax

Dixon et al. 1976

Borax

$\mathrm{M}$ (infertility for 5 weeks

following exposure)

organ weights,

esticular enzym

activities, testicular

histopathology, ability

to impregnate

non-treated females. 
Table 3-2 Levels of Significant Exposure to Boron - Oral

\begin{tabular}{|c|c|c|}
\hline $\begin{array}{l}\text { Key to } \\
\text { Figure }\end{array}$ & $\begin{array}{l}\text { Species } \\
\text { (Strain) }\end{array}$ & $\begin{array}{l}\text { Exposu } \\
\text { Duratio } \\
\text { Frequen } \\
\text { (Route }\end{array}$ \\
\hline 42 & $\begin{array}{l}\text { Rat } \\
\text { (Wistar) }\end{array}$ & $\begin{array}{l}1 \mathrm{x} / \mathrm{d} \\
4 \mathrm{wk} \\
(\mathrm{G})\end{array}$ \\
\hline
\end{tabular}

(G)

$43 \quad$ Rat 9 wk

(Fischer- 344) (F)

$44 \quad$ Rat

(Wistar) $\quad 4 \mathrm{wk}$

\section{$x / d$}

(G)

LOAEL

NOAEL Less Serious Serious

(mg/kg/day)

Reference

Chemical Form

Comments

System (mg/kg/day) (mg/kg/day)

$53 \mathrm{M} \mathrm{(13}$ and 15\% reduction in Fukuda et al. 2000

absolute and relative

Boric acid

testes weight; cellula

Boric acid

debris in the testes,

cauda and caput

epididymis; focal atrophy

of the seminiferous

tubules; decrease in the

number of sperm in the

ducta lumina)

26 M (mildly inhibited

spermiation by week 5)

$52 \mathrm{M}$ (testicular atrophy by

week 9; severe inhibition

of spermiation)

Ku et al. 1993a

Boric acid

Kudo et al. 2000

Boric acid
Endpoints: male

reproductive organ

weights and

histopathology.

Endpoints: male reproductive organ weights and

histopathology, sperm morphology.

Endpoints: male reproductive organ weights,

histopathology, and sperm morphology. 
Table 3-2 Levels of Significant Exposure to Boron - Oral

\begin{tabular}{|c|c|c|}
\hline $\begin{array}{l}\text { Key to } \\
\text { Figure }\end{array}$ & $\begin{array}{l}\text { Species } \\
\text { (Strain) }\end{array}$ & $\begin{array}{r}\text { Exposu } \\
\text { Duratio } \\
\text { Frequen } \\
\text { (Route }\end{array}$ \\
\hline 45 & Rat & $\begin{array}{l}30-60 \\
(F)\end{array}$ \\
\hline 46 & $\begin{array}{l}\text { Rat } \\
\text { (Sprague- } \\
\text { Dawley) }\end{array}$ & $\begin{array}{l}60 \mathrm{~d} \\
(\mathrm{~F})\end{array}$ \\
\hline
\end{tabular}

LOAEL

rationl

(Route)

NOAEL

Less Serious

LOAEL

System (mg/kg/day) (mg/kg/day)

(mglkg/day)

Reference

Chemical Form

Comments

$30-60 d$

(F)

50

$60 d$

$4 \quad$ Rat $70 d$

(Long- Evans) (W)

$48 \quad$ Rat

$\begin{array}{ll}\text { Rat } & 90 \mathrm{~d} \\ \text { (Sprague- } & (\mathrm{F})\end{array}$

Dawley)

49 Rat

$90 \mathrm{~d}$

(Sprague-

(F)

Dawley)
100 (testicular atrophy, decreased enzymes)

Lee et al. 1978

Borax

$136 \mathrm{M}$ (decreased weights of testes and associate tissues; decreased
sperm motility, number of

spermatocytes,

spermatids, Leydig cells,

testosterone levels,

sexually aggressive

behavior, number of

females impregnated;

increased fetal resorption

in impregnated females)

44.7 M (impaired

spermatogenesis)

$45 \mathrm{M} \quad 150 \mathrm{M}(53 \%$ reduction in

testicular weight)

$150 \mathrm{M}(53 \%$ decrease in

testicular weight;

testicular atrophy)
Nusier and Bataineh 2005

Boric acid

Endpoints: male reproductive organ weights,

histopathology, sperm morphology, plasma

$\mathrm{FSH}$ and testosterone

levels, sexual behavior.

Seal and Weeth 1980

Borax

Weir and Fisher 1972

Borax

Weir and Fisher 1972

Borax 
Table 3-2 Levels of Significant Exposure to Boron - Oral

(continued)

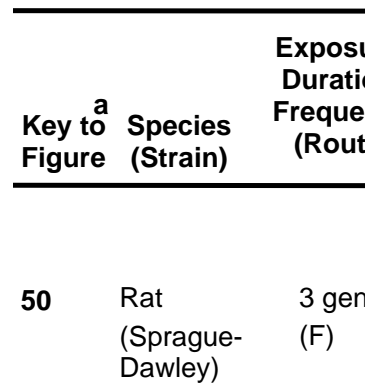

51

Rat

(Sprague-

Dawley)

3 gen

(F)

$52 \quad$ Rat

(Sprague- 3 ge

Dawley)

53 Rat

Dawley)

LOAEL

NOAEL Less Serious

(mg/kg/day)

(mg/kg/day)

Reference

Chemical Form

Comments

(

$\begin{array}{ll}101^{\mathrm{C}} \mathrm{M} \text { (complete sterility) } & \text { Weir and Fisher } 1972 \\ 116 \mathrm{~F} \text { (complete sterility) } & \text { Borax }\end{array}$

$101^{\mathrm{C}} \mathrm{M}$ (complete sterility)

$116 \mathrm{~F}$ (complete sterility)

$116 \mathrm{~F}$ (complete sterility)

$116 \mathrm{~F}$ (complete sterility)

116 F (complete sterility)

Weir and Fisher 1972 Boric acid Weir and Fisher 1972
Borax

Weir and Fisher 1972 Boric acid
Both genders exposed Sterility associated with testicular atrophy in males and decreased ovulation in females.

Both genders exposed. Sterility associated with

testicular atrophy in

males and decreased

ovulation in females.

Exposed females mated with

non-exposed males;

females showed

decreased ovulation.

Exposed females mated with

non-exposed males;

females showed

decreased ovulation 
Table 3-2 Levels of Significant Exposure to Boron - Oral

\begin{tabular}{lll}
\hline & & $\begin{array}{c}\text { Exposu } \\
\text { Duration }\end{array}$ \\
$\begin{array}{l}\text { Key to } \\
\text { Figure }\end{array}$ & $\begin{array}{r}\text { Species } \\
\text { (Strain) }\end{array}$ & $\begin{array}{r}\text { Frequen } \\
\text { (Route) }\end{array}$ \\
\hline & & \\
54 & $\begin{array}{l}\text { Rat } \\
\text { (Wistar) }\end{array}$ & $\begin{array}{l}3 \mathrm{wk} \\
\text { (G) }\end{array}$
\end{tabular}

$\begin{array}{ll}\text { Mouse } & 27 \mathrm{wk} \\ (\mathrm{CD}-1) & \text { (F) }\end{array}$

$(\mathrm{CD}-1)$

(F)

56 Mouse

M: $17 d(d ~ 3-20)$
$F: 20 d(d ~ 0-20)$

(G)

57 Mouse

13 wk

(B6C3F1)
(F)

LOAEL

NOAEL

Less Serious

(mg/kg/day)

System

(mg/kg/day)

g/day)

$9 \mathrm{M}$

$21 \mathrm{M}$ $70 \mathrm{M}$ (reduced testis weight,
germ cell loss)
$26 \mathrm{M}$ (increased

pre-implantation fetal

loss; decreased sperm

motility;

morphologically-abnorma

sperm heads and tails)

$111 \mathrm{M}$ (degeneration of the seminiferous tubules; impaired

spermatogenesis;

decreased sperm

motility, litters per mating

pair, and live pups per

litter)
$210 \mathrm{M}$ (exfoliation/disruption of Harris et al. 1992
seminiferous tubules:
inhibited spermiation;
Boric acid
effect on $\%$ females
pregnant)

Fail et al. 1991

Boric acid

oshizaki et al. 1999

Boric acid

Bolic acid

288 M (degeneration or atrophy NTP 1987, Dieter 1994 of seminiferous tubules) Boric acid
Comments

Exposed males mated

with unexposed

females; inability to

impregnate females at

$88 \mathrm{mg} / \mathrm{kg} /$ day.

Treated males mated with untreated females. Complete sterility at

$221 \mathrm{mg}$ boron/kg/day

Mating on days 8-12;

Endpoints: male

reproductive organ

weight and histology; \%

pregnant and number

of live implants. 
Table 3-2 Levels of Significant Exposure to Boron - Oral

\begin{tabular}{lll}
\hline & & $\begin{array}{c}\text { Exposu } \\
\text { Duration }\end{array}$ \\
$\begin{array}{l}\text { Key to } \\
\text { Figure }\end{array}$ & $\begin{array}{l}\text { Species } \\
\text { (Strain) }\end{array}$ & $\begin{array}{r}\text { Freque } \\
\text { (Route) }\end{array}$ \\
\hline & & \\
58 & $\begin{array}{l}\text { Dog } \\
\text { (Beagle) }\end{array}$ & $\begin{array}{l}\text { (F) d } \\
\text { (B) }\end{array}$
\end{tabular}

$59 \quad$ Dog

(F)

\section{Developmental}

60 Rat

(Sprague-

Dawley)

61 Rat

(Sprague-

Dawley)

$62 \quad$ Mouse

(CD-1)

(F)

(F)

(F)
Gd 0-20

$6 \mathrm{M}$

$6 \mathrm{M}$

13.6 ${ }^{d}$ (reduced fetal weight)

$10 \mathrm{~F}$

$0-20$

(reduced fetal weight

increase in skeletal

abnormalities observed

on gestation day 20)

Gd 0-17

LOAEL

Reference

Chemical Form

Comments

(mg/kg/day)

60.5 M (50\% decrease in relative Weir and Fisher 1972

testes weight; severe Borax

testicular atrophy)

60.5 M (40\% decrease in testes Weir and Fisher 1972 weight; severe testicular Boric acid atrophy)

Price et al. 1996a, 1998 Boric acid

(rib cage defects, ventricles of the brain ncreased resorptions)

Effects seen on gestation day 20 were ed on postnatal day 21 
Table 3-2 Levels of Significant Exposure to Boron - Oral

(continued)

\begin{tabular}{|c|c|c|}
\hline $\begin{array}{l}\text { Key to } \\
\text { Figure }\end{array}$ & $\begin{array}{l}\text { Species } \\
\text { (Strain) }\end{array}$ & $\begin{array}{c}\text { Exposure } \\
\text { Durationl } \\
\text { Frequency } \\
\text { (Route) }\end{array}$ \\
\hline \multicolumn{3}{|c|}{$\begin{array}{l}\text { CHRONIC EXPOSURE } \\
\text { Death }\end{array}$} \\
\hline 63 & $\begin{array}{l}\text { Mouse } \\
\text { (B6C3F1) }\end{array}$ & $\begin{array}{l}103 w k \\
(F)\end{array}$ \\
\hline
\end{tabular}

LOAEL

\begin{tabular}{lcl}
\multicolumn{2}{c}{ LOAEL } & \\
\hline Less Serious & Serious & Reference \\
$(\mathrm{mg} / \mathrm{kg} /$ day $)$ & $(\mathrm{mg} / \mathrm{kg} /$ day $)$ & Chemical Form
\end{tabular}

System (mg/kg/day) (mg/kg/day)

(mg/kg/day)

Comments

Death

\begin{tabular}{|c|c|c|c|}
\hline \multirow[t]{4}{*}{64} & \multirow[t]{4}{*}{$\begin{array}{l}\text { Rat } \\
\text { (Sprague- } \\
\text { Dawley) }\end{array}$} & \multirow[t]{4}{*}{$\begin{array}{l}2 \mathrm{yr} \\
\text { (F) }\end{array}$} & Resp \\
\hline & & & Cardio \\
\hline & & & Gastro \\
\hline & & & Hemato \\
\hline
\end{tabular}

(B6C3F1) (F)

Systemic
$64 \quad \mathrm{Ra}$

(Sprague- $\quad(\mathrm{F})$

Hemato

$24 \mathrm{~F}$

Hepatic

81

Renal

81

Endocr

81

Dermal

24

Ocular

$\mathrm{Bd} \mathrm{Wt}$

24

24
$81 \mathrm{~F}$ (decreased packed cell volume and hemoglobin levels)

79 (40\% mortality)

NTP 1987, Dieter 1994

Boric acid

Weir and Fisher 1972

Boric acid

NOAELS are for histopathology.
81 (scaly tails,

desquamation of skin on

footpads)

81 (bloody ocular discharge)

81 (reduced growth

throughout study) 
Table 3-2 Levels of Significant Exposure to Boron - Oral

(continued)

\begin{tabular}{|c|c|c|c|c|}
\hline $\begin{array}{l}\text { Key to } \\
\text { Figure }\end{array}$ & $\begin{array}{l}\text { Species } \\
\text { (Strain) }\end{array}$ & $\begin{array}{c}\text { Exposurel } \\
\text { Duration/ } \\
\text { Frequency } \\
\text { (Route) }\end{array}$ & System & $\begin{array}{r}\text { NOAE } \\
(\mathrm{mg} / \mathrm{kg}\end{array}$ \\
\hline \multirow[t]{4}{*}{65} & $\begin{array}{l}\text { Rat } \\
\text { (Sprague- } \\
\text { Dawley) }\end{array}$ & $\begin{array}{l}2 \mathrm{yr} \\
(\mathrm{F})\end{array}$ & Resp & 81 \\
\hline & & & Cardio & 81 \\
\hline & & & Gastro & 81 \\
\hline & & & Hemato & 24 \\
\hline
\end{tabular}

LOAEL

Reference

Less Serious

Serious

Chemical Form

Comments

81 (decreased packed cell volume and hemoglobin levels)

Hepatic 81

Renal 81

Endocr 81

Dermal 24

81 (scaly tails,

desquamation of skin on

footpads)

$\begin{array}{ll}\text { Weir and Fisher } 1972 & \text { NOAELs are for } \\ \text { histopathology. }\end{array}$

Borax

histopathology.

$\begin{array}{llll}\text { Ocular } & 24 & 81 & \text { (bloody ocular discharge) } \\ \text { Bd Wt } & 24 & 81 & \begin{array}{l}\text { (decreased growth } \\ \text { throughout study) }\end{array}\end{array}$


Table 3-2 Levels of Significant Exposure to Boron - Oral

(continued)

\begin{tabular}{|c|c|c|c|c|c|c|c|c|}
\hline \multirow[b]{2}{*}{$\begin{array}{l}\text { Key to } \\
\text { Figure }\end{array}$} & \multirow[b]{2}{*}{$\begin{array}{l}\text { Species } \\
\text { (Strain) }\end{array}$} & \multirow{2}{*}{$\begin{array}{l}\text { Exposurel } \\
\text { Duration/ } \\
\text { Frequency } \\
\text { (Route) }\end{array}$} & \multirow[b]{2}{*}{ System } & \multirow[b]{2}{*}{$\begin{array}{l}\text { NOAEL } \\
\text { (mg/kg/day) }\end{array}$} & \multicolumn{2}{|c|}{ LOAEL } & \multirow[b]{2}{*}{ Reference } & \multirow[b]{2}{*}{ Comments } \\
\hline & & & & & $\begin{array}{r}\text { Less Serious } \\
\text { (mg/kg/day) }\end{array}$ & $\begin{array}{l}\text { Serious } \\
\text { (mg/kg/day) }\end{array}$ & & \\
\hline
\end{tabular}

$\begin{array}{llr}\begin{array}{ll}103 \text { wk } \\ \text { (F) }\end{array} & \text { Resp } & 79 \mathrm{~F} \\ & \text { Cardio } & 201 \\ & \text { Gastro } & 201 \\ & \text { Hemato } & \\ & \text { Hepatic } & \\ & \\ & \\ & \text { Renal } & 201 \\ \text { Endocr } & 201 \\ \text { Ocular } & 201 \\ \text { Bd Wt } & 79\end{array}$

$201 \mathrm{~F}$ (lung hemorrhage)

NTP 1987, Dieter 1994

NOAELS are for

(B6C3F1) (F)

$79 \mathrm{M}$ (spenic hematopoiesis)

79 (chronic inflammation; coagulative necrosis)

$201 \quad(10 \%-17 \%$ decrease in

body weight) 
Table 3-2 Levels of Significant Exposure to Boron - Oral

\begin{tabular}{|c|c|c|c|c|c|c|c|c|}
\hline \multirow[b]{2}{*}{$\begin{array}{l}\text { Key to } \\
\text { Figure }\end{array}$} & \multirow[b]{2}{*}{$\begin{array}{l}\text { Species } \\
\text { (Strain) }\end{array}$} & \multirow{2}{*}{$\begin{array}{l}\text { Exposurel } \\
\text { Durationl } \\
\text { Frequency } \\
\text { (Route) }\end{array}$} & \multirow[b]{2}{*}{ System } & \multirow[b]{2}{*}{$\begin{array}{l}\text { NOAEL } \\
\text { (mg/kg/day) }\end{array}$} & \multicolumn{3}{|c|}{ LOAEL } & \multirow[b]{2}{*}{ Comments } \\
\hline & & & & & $\begin{array}{r}\text { Less Serious } \\
(\mathrm{mg} / \mathrm{kg} / \mathrm{day})\end{array}$ & $\begin{array}{l}\text { Serious } \\
\text { (mg/kg/day) }\end{array}$ & $\begin{array}{l}\text { Reference } \\
\text { Chemical Form }\end{array}$ & \\
\hline
\end{tabular}

\section{Dog} (Beagle)

$\begin{array}{lll}\begin{array}{l}2 \mathrm{yr} \\ (\mathrm{F})\end{array} & \text { Resp } & 6.8 \\ & \text { Cardio } & 6.8 \\ & \text { Gastro } & 6.8 \\ & \text { Hemato } & 6.8 \\ & \text { Musc/skel } & 6.8 \\ & \text { Hepatic } & 6.8 \\ & \text { Renal } & 6.8 \\ & \text { Endocr } & 6.8 \\ & \text { Dermal } & 6.8 \\ & \text { Ocular } & 6.8 \\ & \text { Bd Wt } & 6.8\end{array}$


Table 3-2 Levels of Significant Exposure to Boron - Oral

(continued)

\begin{tabular}{|c|c|c|c|c|}
\hline $\begin{array}{l}\text { Key to } \\
\text { Figure }\end{array}$ & $\begin{array}{r}\text { Species } \\
\text { (Strain) }\end{array}$ & $\begin{array}{c}\text { Exposurel } \\
\text { Duration/ } \\
\text { Frequency } \\
\text { (Route) }\end{array}$ & System & $\begin{array}{r}\text { NOAE } \\
(\mathrm{mg} / \mathrm{kg} / \\
\end{array}$ \\
\hline \multirow[t]{11}{*}{68} & $\begin{array}{l}\text { Dog } \\
\text { (Beagle) }\end{array}$ & $\begin{array}{l}2 \mathrm{yr} \\
(\mathrm{F})\end{array}$ & Resp & 6.8 \\
\hline & & & Cardio & 6.8 \\
\hline & & & Gastro & 6.8 \\
\hline & & & Hemato & 6.8 \\
\hline & & & Musc/skel & 6.8 \\
\hline & & & Hepatic & 6.8 \\
\hline & & & Renal & 6.8 \\
\hline & & & Endocr & 6.8 \\
\hline & & & Dermal & 6.8 \\
\hline & & & Ocular & 6.8 \\
\hline & & & $\mathrm{Bd} W \mathrm{t}$ & 6.8 \\
\hline
\end{tabular}

LOAEL

(mg/kg/day)

(mg/kg/day)

Reference

Chemical Form

Comments

\section{Neurological}

69 Rat

(Sprague-

Dawley)

70 Rat

(Sprague-

Dawley)

71 Mouse

(B6C3F1)

103 wk

(F)
24

24

81 (increased relative brain weight)

201
Weir and Fisher 1972

Borax

Weir and Fisher 1972

Boric acid

Weir and Fisher 1972

Borax

NTP 1987, Dieter 1994 Boric acid
NOAELs are for histopathology.

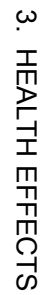


Table 3-2 Levels of Significant Exposure to Boron - Oral

\begin{tabular}{|c|c|c|}
\hline $\begin{array}{l}\text { Key to } \\
\text { Figure }\end{array}$ & $\begin{array}{l}\text { Species } \\
\text { (Strain) }\end{array}$ & $\begin{array}{l}\text { Exposu } \\
\text { Duratic } \\
\text { Frequer } \\
\text { (Rout }\end{array}$ \\
\hline \multicolumn{3}{|c|}{ Reproductive } \\
\hline 72 & Human & $\begin{array}{l}\text { NS } \\
\text { (Ens }\end{array}$ \\
\hline 73 & $\begin{array}{l}\text { Rat } \\
\text { (Sprague- } \\
\text { Dawley) }\end{array}$ & $\begin{array}{l}2 \mathrm{yr} \\
(\mathrm{F})\end{array}$ \\
\hline
\end{tabular}

LOAEL

urationl

(Rouency

LOAEL

System (mg/kg/day) (mg/kg/day)

(mg/kg/day)

Reference

Chemical Form

Comments

74 Rat

(Sprague- (F)

Dawley)

$75 \quad$ Mouse

(F)

76

$\begin{array}{ll}\text { Dog } & 2 \mathrm{yr} \\ \text { (Beagle) } & (\mathrm{F})\end{array}$

$6.1 \mathrm{~F}$

$24 \mathrm{M}$

$6.8 \mathrm{M}$

\section{Liu et al. 2006a}

Borate

$81 \mathrm{M}$ (decreased relative testes weight; testicular

atrophy; atrophied

seminiferous epithelium

and reduced tubular size)

Weir and Fisher 1972

Borax

$81 \mathrm{M}$ (decreased relative testes weight; testicular

atrophy; atrophied

seminiferous epithelium

and reduced tubular size)

Weir and Fisher 1972

Boric acid

201 (testicular atrophy,

interstitial hyperplasia)

NTP 1987, Dieter 1994

Boric acid

Weir and Fisher 1972

Borax 
Table 3-2 Levels of Significant Exposure to Boron - Oral

\begin{tabular}{|c|c|c|c|c|c|c|c|}
\hline \multirow[b]{2}{*}{$\begin{array}{l}\text { Key to } \\
\text { Figure }\end{array}$} & \multirow[b]{2}{*}{$\begin{array}{l}\text { Species } \\
\text { (Strain) }\end{array}$} & \multirow{2}{*}{$\begin{array}{l}\text { Exposurel } \\
\text { Duration/ } \\
\text { Frequency } \\
\text { (Route) }\end{array}$} & \multirow[b]{2}{*}{ System } & \multirow[b]{2}{*}{$\begin{array}{l}\text { NOAEL } \\
\text { (mg/kg/day) }\end{array}$} & \multicolumn{2}{|c|}{ LOAEL } & \multirow[b]{2}{*}{$\begin{array}{l}\text { Reference } \\
\text { Chemical Form }\end{array}$} \\
\hline & & & & & $\begin{array}{r}\text { Less Serious } \\
(\mathrm{mg} / \mathrm{kg} / \text { day })\end{array}$ & $\begin{array}{l}\text { Serious } \\
\text { (mg/kg/day) }\end{array}$ & \\
\hline 77 & $\begin{array}{l}\text { Dog } \\
\text { (Beagle) }\end{array}$ & $\begin{array}{l}2 \mathrm{yr} \\
(\mathrm{F})\end{array}$ & & $6.8 \mathrm{M}$ & & & $\begin{array}{l}\text { Weir and Fisher } 1972 \\
\text { Boric acid }\end{array}$ \\
\hline
\end{tabular}

\section{Table 3-2 Oral}

a The number corresponds to entries in Figure 3-2.

b Used to derive an acute-duration oral MRL of $0.2 \mathrm{mg} / \mathrm{kg} /$ day; dose divided by an uncertainty factor of 100 (10 for interspecies extrapolation and 10 for human variability).

c Differences in levels of health effects and cancer effects between male and females are not indicated in Figure 3-2. Where such differences exist, only the levels of effect for the most sensitive gender are presented.

d Used to derive an intermediate-duration oral MRL of $0.2 \mathrm{mg} / \mathrm{kg} /$ day; a point of departure from benchmark dose analysis of $10.3 \mathrm{mg}$ boron/ $\mathrm{kg} / \mathrm{day}$ was divided by a chemical-specific uncertainty factor of 66 (3.3 for toxicokinetic extrapolation from animals to humans, 3.16 for toxicodynamic extrapolation from animals to humans, 2.0 for variability in human toxicokinetics, and 3.16 for variability in human toxicodynamics).

$\mathrm{Bd} \mathrm{Wt}=$ body weight; $(\mathrm{C})=$ capsule; Cardio = cardiovascular; $\mathrm{CEL}=$ cancer effect level; $\mathrm{d}=$ day $(\mathrm{s})$; Endocr $=$ endocrine; $(\mathrm{F})=$ feed; $\mathrm{F}=\mathrm{Female} ; \mathrm{FSH}=$ follicle stimulating hormone; $(\mathrm{G})$ = gavage; Gastro = gastrointestinal; Gd = gestational day; Hemato = hematological; $L D 50=$ lethal dose, $50 \%$ kill; LOAEL = lowest-observed-adverse-effect level; $M=$ male;

Metab = metabolic; Musc/skel = musculoskeletal; NOAEL = no-observed-adverse-effect level; NS = not specified; Resp = respiratory; $\mathrm{x}=$ time $(\mathrm{s}) ;(\mathrm{W})=\mathrm{drinking}$ water; $\mathrm{wk}=$ week(s); $\mathrm{yr}=$ year $(\mathrm{s})$ 
Figure 3-2 Levels of Significant Exposure to Boron - Oral

Acute $(\leq 14$ days)

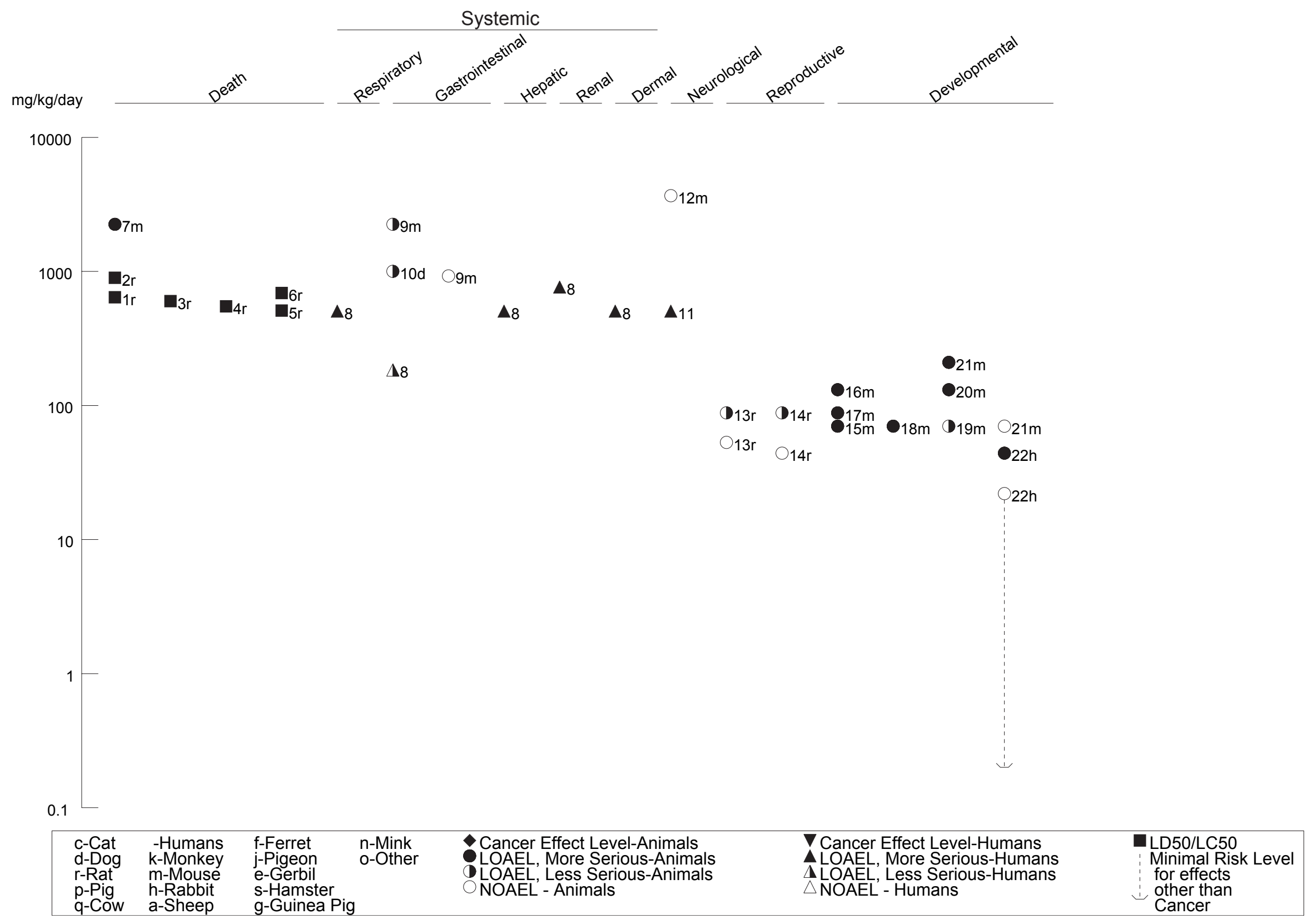


Figure 3-2 Levels of Significant Exposure to Boron - Oral (Continued)

Intermediate (15-364 days)

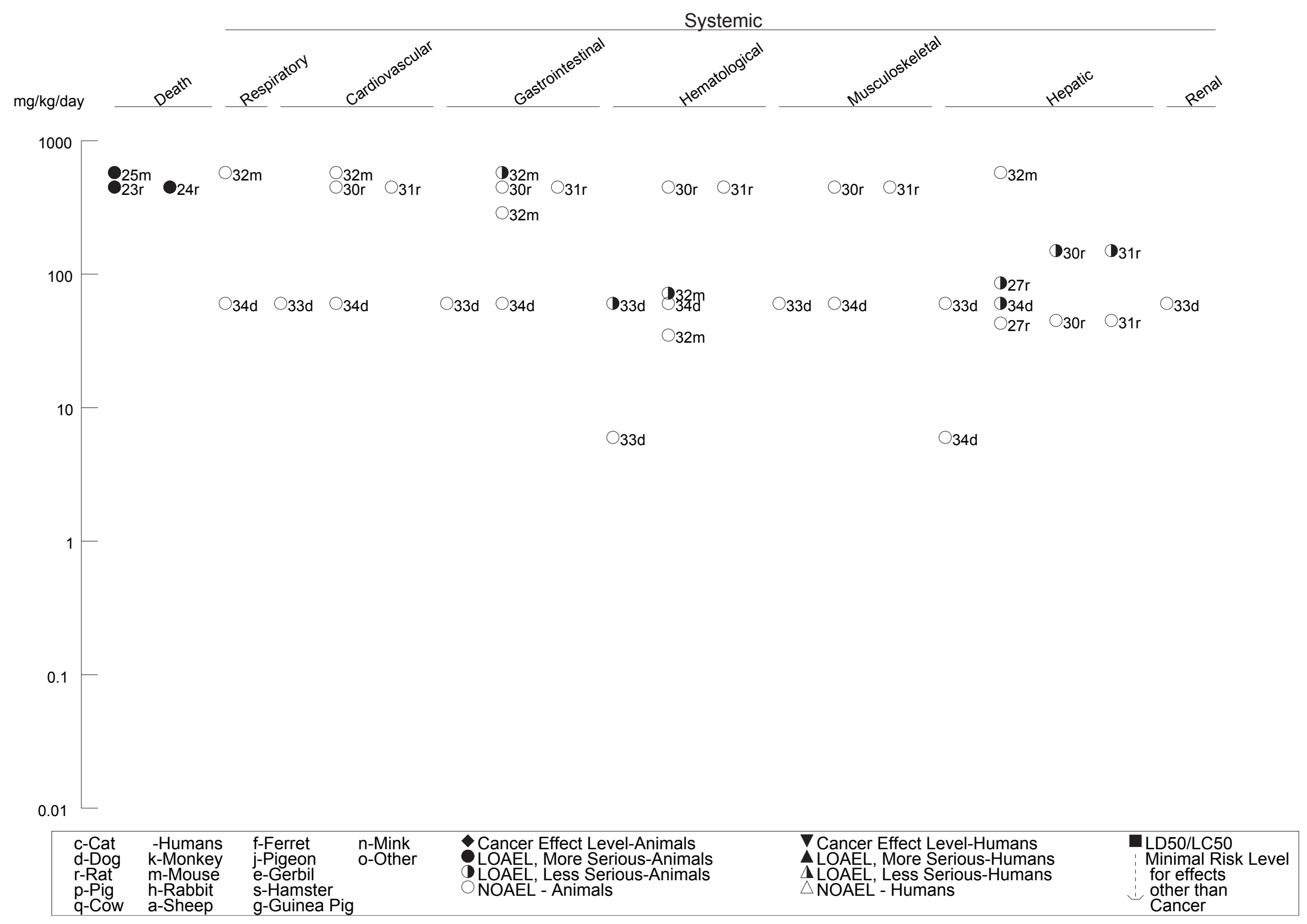


Figure 3-2 Levels of Significant Exposure to Boron - Oral (Continued)

Intermediate (15-364 days)

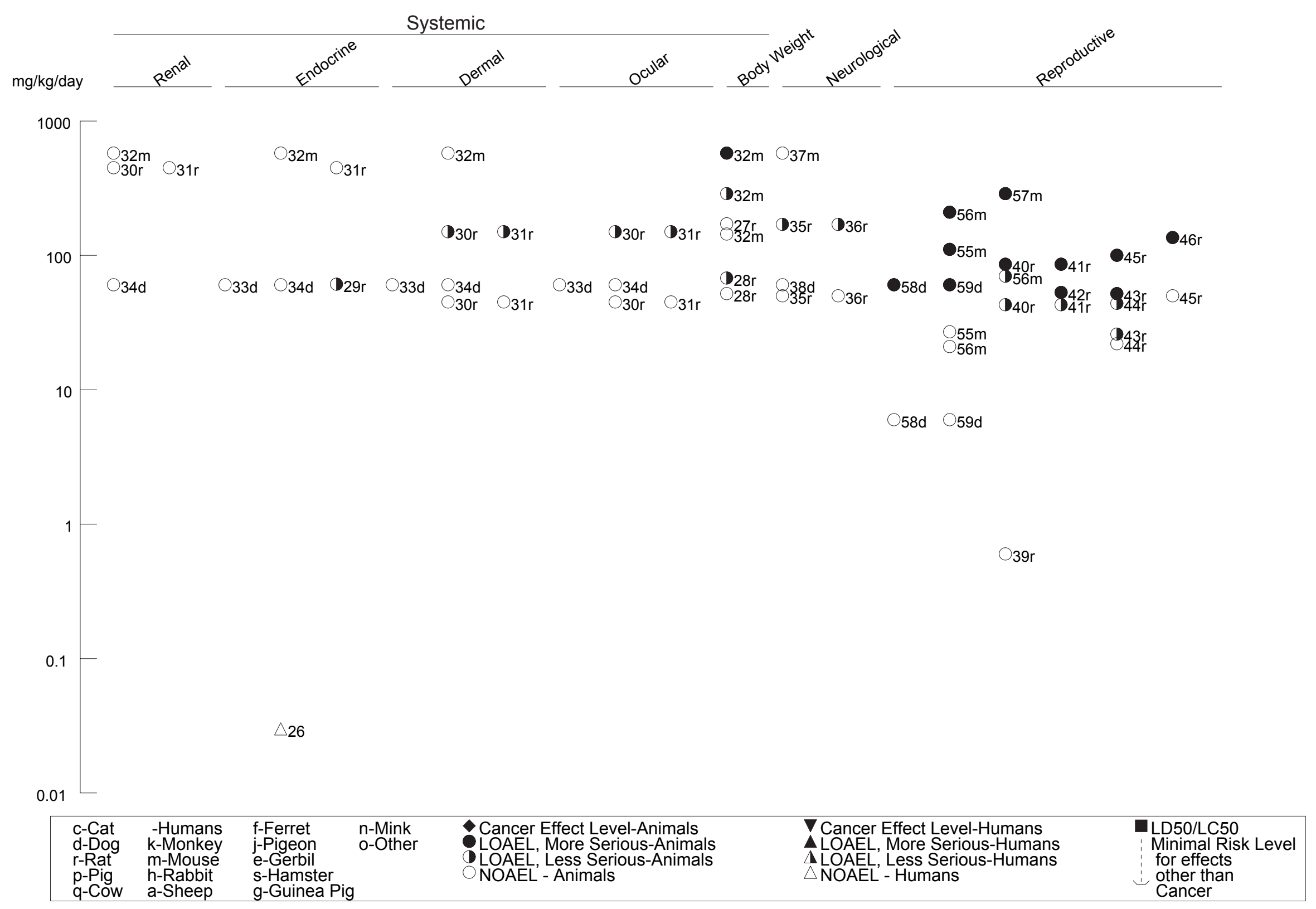


Figure 3-2 Levels of Significant Exposure to Boron - Oral (Continued)

Intermediate (15-364 days)

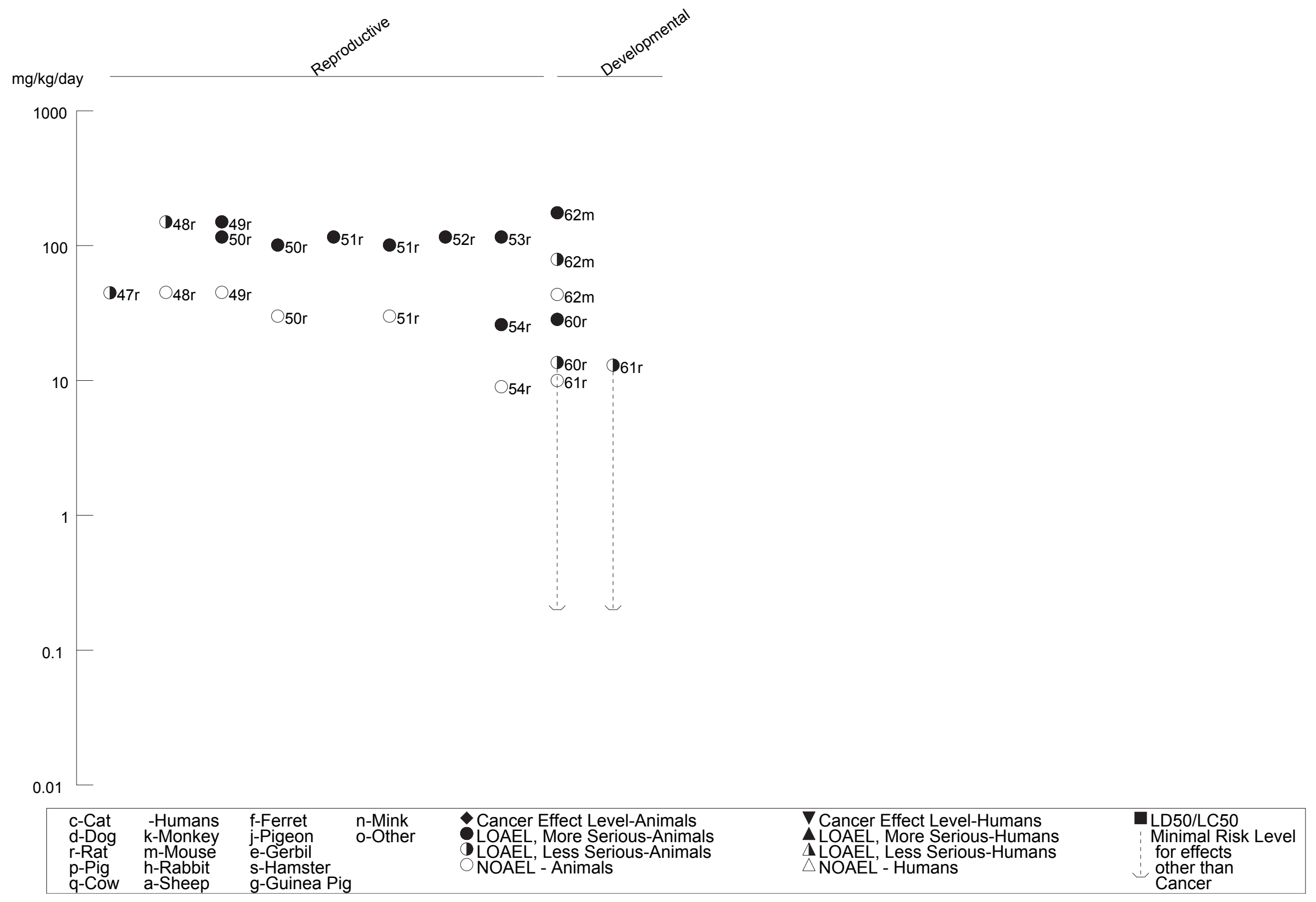


Figure 3-2 Levels of Significant Exposure to Boron - Oral (Continued)

Chronic ( $\geq 365$ days)

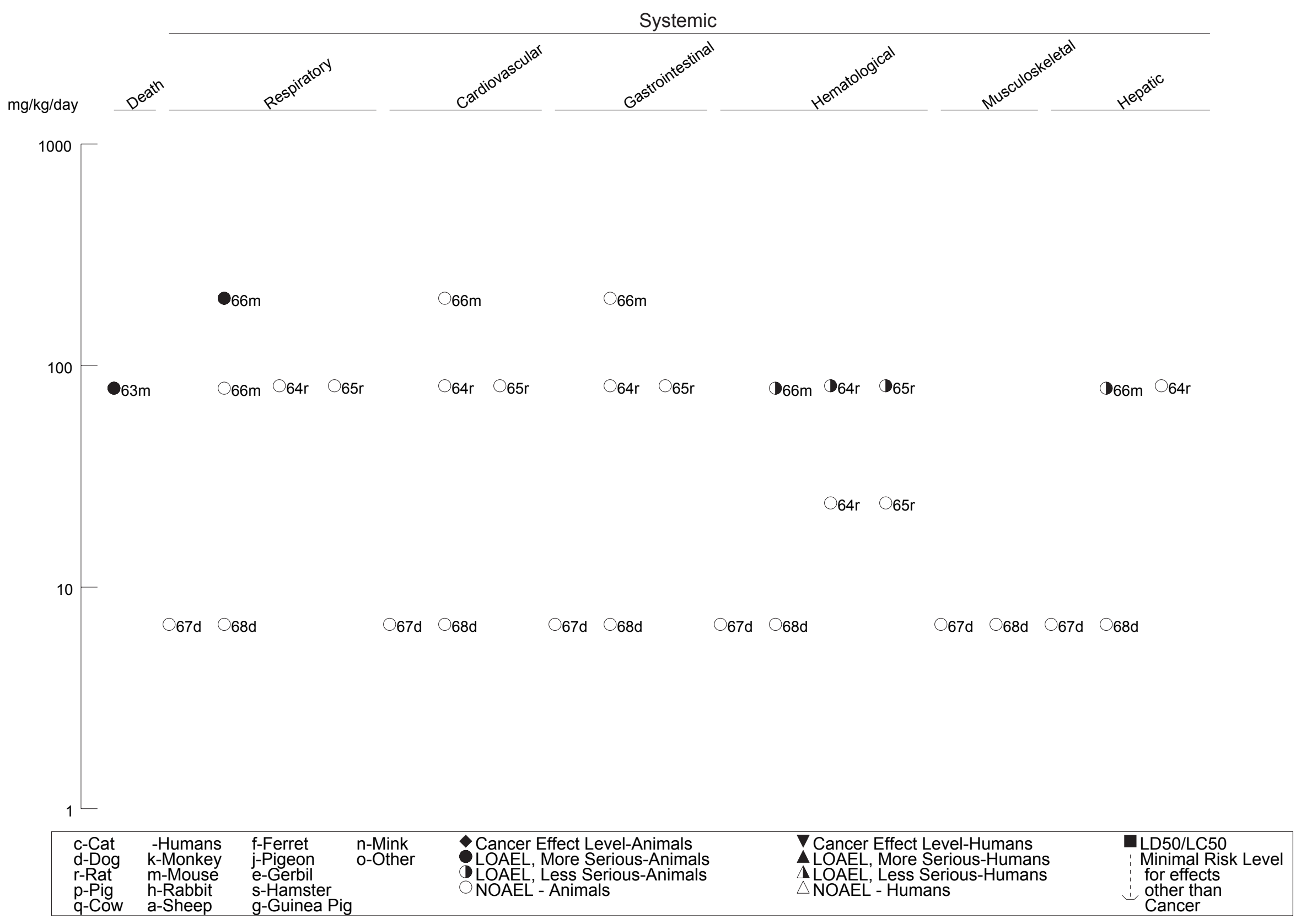


Figure 3-2 Levels of Significant Exposure to Boron - Oral (Continued)

Chronic ( $\geq 365$ days)

\section{Systemic}

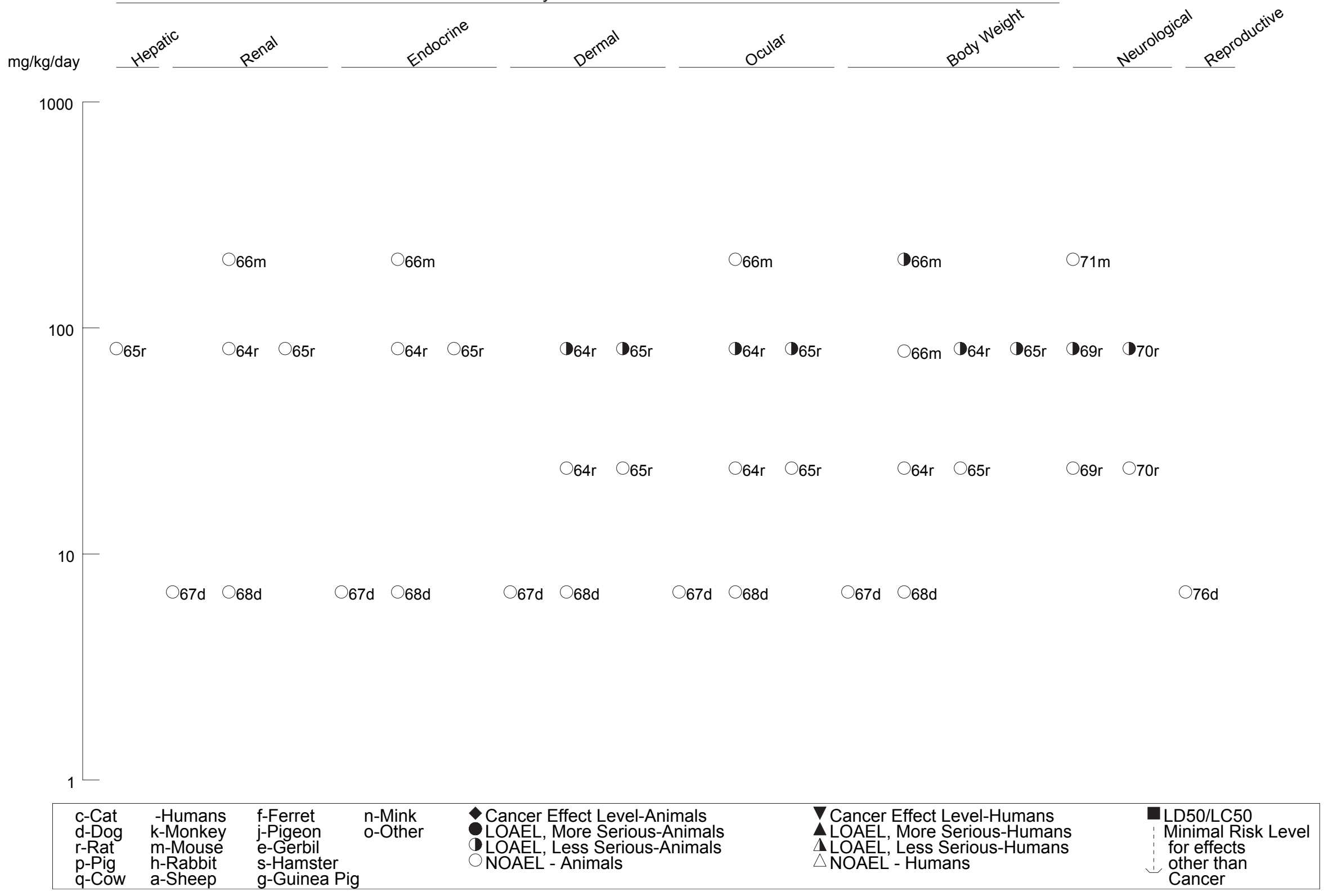


Figure 3-2 Levels of Significant Exposure to Boron - Oral (Continued)

Chronic ( $\geq 365$ days)
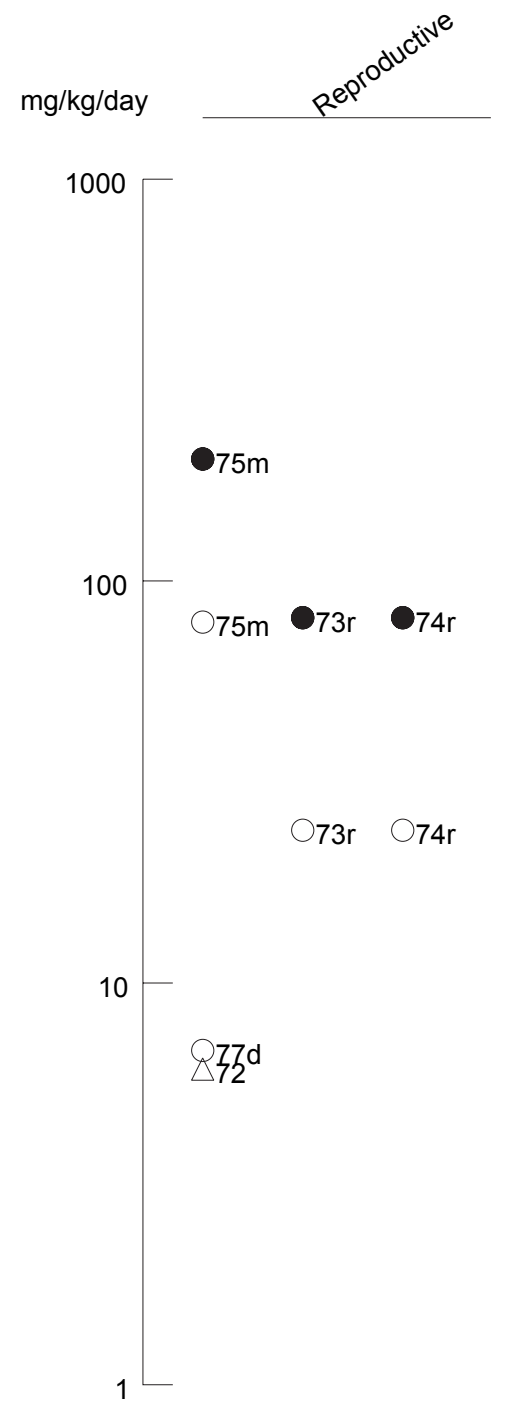

\begin{tabular}{|c|c|c|c|c|c|c|}
\hline $\begin{array}{l}\text { c-Cat } \\
\text { d-Dog } \\
\text { r-Rat } \\
\text { p-Pig } \\
\text { q-Cow }\end{array}$ & $\begin{array}{l}\text {-Humans } \\
\text { k-Monkey } \\
\text { m-Mouse } \\
\text { h-Rabbit } \\
\text { a-Sheep }\end{array}$ & $\begin{array}{l}\text { f-Ferret } \\
\text { j-Pigeon } \\
\text { e-Gerbil } \\
\text { s-Hamster } \\
\text { g-Guinea Pig }\end{array}$ & $\begin{array}{l}\text { n-Mink } \\
\text { o-Other }\end{array}$ & $\begin{array}{l}\text { Cancer Effect Level-Animals } \\
\text { LOAEL, More Serious-Animals } \\
\text { LOAEL, Less Serious-Animals } \\
\text { NOAEL - Animals }\end{array}$ & $\begin{array}{l}\nabla \text { Cancer Effect Level-Humans } \\
\text { A LOAEL, More Serious-Humans } \\
\Delta \text { LOAEL, Less Serious-Humans } \\
\triangle \text { NOAEL - Humans }\end{array}$ & $\begin{array}{l}\text { LD50/LC50 } \\
\text { Minimal Risk Level } \\
\text { for effects } \\
\text { other than } \\
\text { Cancer }\end{array}$ \\
\hline
\end{tabular}


In B6C3F1 mice, dietary exposure to $\geq 2,251 \mathrm{mg}$ boron/kg/day as boric acid for 14 days resulted in gastric hyperplasia and dysplasia (NTP 1987). Dogs given a single dose of $1,000 \mathrm{mg}$ boron/kg as boric acid vomited (Weir and Fisher 1972).

Hematological Effects. In animals orally exposed for intermediate or chronic durations, effects on hematological end points have been observed sporadically in dogs and consistently in mice; they did not occur in rats exposed for 90 days. Mongrel dogs fed $60.5 \mathrm{mg}$ boron/kg/day for 90 days in the diet as borax (but not as boric acid) had decreased packed cell volume and hemoglobin values, but no hematological effects were seen in dogs fed $81 \mathrm{mg}$ boron/kg/day as borax or boric acid for 2 years (Weir and Fisher 1972). Erythrocyte count and total and differential leukocyte counts were comparable to control levels (Weir and Fisher 1972). Splenic extramedullary hematopoiesis occurred in mice fed $72 \mathrm{mg}$ boron/kg/day as boric acid for 90 days and 79 mg boron/kg/day for 2 years (Dieter 1994; NTP 1987), while no hematological effects were observed in rats fed $450 \mathrm{mg}$ boron/kg/day as borax or boric acid for 90 days (Weir and Fisher 1972). Decreased packed cell volume and hemoglobin values were seen in rats fed $81 \mathrm{mg}$ boron/kg/day as borax for 2 years (Weir and Fisher 1972).

Hepatic Effects. Case reports in humans suggest that the liver is susceptible to boron toxicity at high dose levels (Wong et al. 1964). Jaundice has been reported, and there were mild alterations at histological examination in infants who ingested 505 or $765 \mathrm{mg}$ boron/kg/day as boric acid (accidentally incorporated in infant formula) for 3-5 days (Wong et al. 1964). In the same incident, congestion and fatty changes were observed, and there was parenchymatous degeneration in newborn infants who ingested 505 or $765 \mathrm{mg}$ boron/kg as boric acid for 3-5 days (Wong et al. 1964). Coagulative necrosis of the liver, proliferative metaplasia of the hepatocytes, giant cell formation, central vein sclerosis, bile stasis, and hepatic steatosis were observed in children ingesting unknown levels of aflatoxin and boric acid in Chinese noodles (Chao et al. 1991a, 1991b).

In mice, the liver appears to be a toxicity target of repeated oral exposure to boric acid, but it is not a target in rats and dogs repeatedly exposed to boric acid or borates. Two-year dietary exposures of $\geq 79 \mathrm{mg}$ boron/kg/day as boric acid to B6C3F1 mice resulted in chronic inflammation and coagulative necrosis in the liver (NTP 1987; Dieter 1994), but no exposure-related hepatic lesions were found in Sprague-Dawley rats fed $81 \mathrm{mg}$ boron/kg/day as boric acid or borax in the diet for 2 years or in mongrel dogs fed $6.8 \mathrm{mg}$ boron/kg/day as boric acid or borax in the diet for 2 years (Weir and Fisher 1972). No exposure-related liver lesions were seen in mice fed $577 \mathrm{mg}$ boron/kg/day as boric acid for 13 weeks 
(Dieter 1994; NTP 1987); in Sprague-Dawley rats fed $450 \mathrm{mg}$ boron/kg/day as boric acid or borax for 90 days (Weir and Fisher 1972); or in mongrel dogs fed $60.5 \mathrm{mg}$ boron/kg/day as boric acid or borax for 90 days (Weir and Fisher 1972).

Boron-related changes in liver weights have not been consistently observed in animal studies. Reduced liver weights were observed in rats fed $86 \mathrm{mg}$ boron/kg/day as borax for 30 days (Dixon et al. 1979) and fed $150 \mathrm{mg}$ boron/kg/day as borax or boric acid for 90 days (Weir and Fisher 1972), while increased relative liver weights were seen in mongrel dogs fed $60.5 \mathrm{mg}$ boron $/ \mathrm{kg} /$ day as boric acid for 90 days (Weir and Fisher 1972).

In liver microsomal fractions from rats given approximately $20.8 \mathrm{mg}$ boron/kg/day as borax in drinking water, NADPH-cytochrome $\mathrm{C}$ reductase activity and cytochrome b5 content decreased in the liver microsomal fraction after 10 and 14 weeks of exposure (Settimi et al. 1982). There was also a reduction in the cytochrome P-450 concentration detected at 14 weeks (Settimi et al. 1982). The toxicological significance of these biochemical changes is not clear, especially since intermediate- and chronic-duration feeding studies with Sprague-Dawley rats fed boric acid or borax did not report exposure-related hepatic lesions (Weir and Fisher 1972).

Renal Effects. Human case reports involving high accidental ingestion levels show that boron can cause injury to the kidney. Acute renal failure was observed in a 77-year-old man ingesting $85 \mathrm{mg}$ boron/kg (as $30 \mathrm{~g}$ of boric acid) (Ishii et al. 1993). Degenerative changes in parenchymal cells with oliguria and albuminuria have been demonstrated in two newborn infants after ingestion of 505 and $765 \mathrm{mg}$ boron/kg/day as boric acid in an evaporated milk formula over a period of 3-5 days (Wong et al. 1964). Acute renal tubule necrosis was seen in children dying from ingestion of unknown levels of aflatoxin and boric acid in Chinese noodles (Chao et al. 1991a, 1991b).

No exposure-related renal lesions were observed in Sprague-Dawley rats fed up to $450 \mathrm{mg}$ boron/kg/day as borax or boric acid for 90 days (Weir and Fisher 1972), in mice fed $577 \mathrm{mg}$ boron/kg/day as boric acid for 13 weeks (NTP 1987; Dieter 1994), or in dogs fed up to $60.5 \mathrm{mg}$ boron/kg/day as borax or boric acid for 90 days (Weir and Fisher 1972). With 2 years of dietary exposure, no exposure-related renal lesions were observed in Sprague-Dawley rats fed $81 \mathrm{mg}$ boron/kg/day as borax or boric acid (Weir and Fisher 1972), in B6C3F1 mice fed up to $201 \mathrm{mg}$ boron/kg/day as boric acid (Dieter 1994; NTP 1987), or in mongrel dogs fed $6.8 \mathrm{mg}$ boron/kg/day as borax or boric acid (Weir and Fisher 1972). The available data indicate that the kidney is not a sensitive toxicity target of oral exposure to boric acid or borates. 
Endocrine Effects. Human data for endocrine effects from orally ingested boron are limited to studies of low-dose boron nutritional supplementation. Postmenopausal women ingesting $0.4 \mathrm{mg}$ boron/day (as $3.25 \mathrm{mg}$ sodium borate/day) in the diet had a 3-fold increase in plasma testosterone levels compared to those ingesting $0.03 \mathrm{mg}$ boron/day (as $0.25 \mathrm{mg}$ sodium borate/day) (Nielsen et al. 1987). However, bodybuilders taking daily supplements of $2.5 \mathrm{mg}$ boron (approximately $0.03 \mathrm{mg} / \mathrm{kg}$; calculated using mean body weights from study data) for 7 weeks did not exhibit differences from controls in free or total plasma testosterone levels (Ferrando and Green 1993).

Rats fed diets with $61 \mathrm{mg}$ boron/kg/day as boric acid (9000 ppm) for 28 days had decreases of approximately $60-78 \%$ in plasma testosterone levels beginning on day 4 of exposure (Treinen and Chapin 1991). Follicle stimulating hormone (FSH) and leutenizing hormone (LH) levels in the blood of rats fed 26-68 mg boron/kg/day as boric acid (3,000-9,000 ppm; doses estimated by study authors) in the diet were increased, possibly in response to testicular atrophy observed in the 52 and $68 \mathrm{mg}$ boron/kg/day groups (Ku et al. 1993a). No histologic lesions were observed in thyroid, adrenals, or pituitary tissues of Sprague-Dawley rats fed 450 or $81 \mathrm{mg}$ boron/kg/day as boric acid or borax in the diet for 90 days or 2 years, respectively, or in mongrel dogs fed 60.5 or $6.8 \mathrm{mg}$ boron/ $\mathrm{kg} /$ day as boric acid or borax in the diet for 90 days or 2 years, respectively (Weir and Fisher 1972) or in pituitary tissues of mice fed 577 or $201 \mathrm{mg}$ boron/kg day as boric acid for 90 days or 2 years, respectively (Dieter 1994; NTP 1987).

Dermal Effects. Skin effects can occur following ingestion of boron (as boric acid) in humans. Extensive exfoliative dermatitis began in infants as an erythema involving palms, soles, and buttocks. It eventually became generalized with subsequent bulbous formation, massive desquamation, and sloughing (Wong et al. 1964). These changes were associated with ingestion of $505 \mathrm{mg}$ boron/kg/day; however, skin lesions were lacking following ingestion of $765 \mathrm{mg}$ boron/kg/day. Similarly, extensive erythema with desquamation was observed in an adult who ingested single doses of boric acid powder (Schillinger et al. 1982). The exact amount ingested was not stated. However, $14 \mathrm{~g}$ (equivalent to $22.5 \mathrm{mg}$ boron/ $\mathrm{kg}$ based on $109 \mathrm{~kg}$ body weight) was measured as missing from a container from which the patient admitted consuming half its contents.

In animals studies, skin lesions were observed in Sprague-Dawley rats fed $150 \mathrm{mg}$ boron/kg/day as borax or boric acid in the diet for 90 days (skin desquamations on the paws and tails) or $81 \mathrm{mg}$ boron/kg/day for 2 years (scaly tails and desquamation on footpads) (Weir and Fisher 1972), but dermal lesions were not observed in B6C3F1 mice exposed to boric acid in the diet for 13 weeks or 2 years (Dieter 1994; NTP 
1987) or in mongrel dogs exposed to boric acid or borax in the diet for 90 days or 2 years (Weir and Fisher 1972).

Ocular Effects. There are no reports of ocular effects in humans following oral exposure to humans.

Sprague-Dawley rats fed $150 \mathrm{mg}$ boron/kg/day as borax or boric acid for 90 days exhibited inflammation of the eyes, while $81 \mathrm{mg}$ boron/kg/day as borax or boric acid in the diet for 2 years resulted in bloody ocular discharge (Weir and Fisher 1972). No ocular effects were seen in dogs fed $60.5 \mathrm{mg}$ boron/kg/day as borax or boric acid for 90 days or $6.8 \mathrm{mg}$ boron/kg/day borax or boric acid for 2 years (Weir and Fisher 1972). Likewise, mice fed $201 \mathrm{mg}$ boron/kg/day as boric acid for 2 years exhibited no ocular effects.

Body Weight Effects. Fisher 344 rats fed $68 \mathrm{mg}$ boron/kg/day as boric acid (9,000 ppm) for 9 weeks had 6\% lower body weight gain than controls (Ku et al. 1993a). Male and female B6C3F1 mice fed 288 and $577 \mathrm{mg}$ boron/kg/day as boric acid (0.5\% of diet) for 13 weeks had 17 and 23\% decreased weight gains, respectively, while 2-year exposures to $201 \mathrm{mg}$ boron/kg/day as boric acid resulted in 19\% lower weight gains in both sexes (NTP 1987, Dieter 1994).

\subsubsection{Immunological and Lymphoreticular Effects}

No studies were located regarding immunological effects in humans or animals after oral exposure to boron.

\subsubsection{Neurological Effects}

Case reports in humans have indicated neurological effects after accidental ingestion of high levels of boron (as boric acid). Newborn infants who ingested 4.5-14 g boric acid showed central nervous system involvement manifested by headache, tremors, restlessness, and convulsions followed by weakness and coma (Wong et al. 1964). Histological examination of 2 of 11 infants revealed congestion and edema of brain and meninges with perivascular hemorrhage and intravascular thrombosis at a dose $\geq 505 \mathrm{mg}$ boron/kg/day (Wong et al. 1964). Seizure disorders have been associated with boron exposures (as borax) in infants who ingested 12-120 g borax for 4-10 weeks (O'Sullivan and Taylor 1983) and 9-125 g borax over a period of 5-12 weeks (Gordon et al. 1973). Estimates of boron dose could not be determined since the authors did not provide body weight data. Blood boron levels in the infants exposed who ingested borax ranged from 2.6 to $8.5 \mu \mathrm{g} / \mathrm{mL}$ (O'Sullivan and Taylor 1983). In one infant with a 
seizure disorder who ingested (via pacifier dipped in honey and borax mixture) approximately $125 \mathrm{~g}$ borax over 3 months, the blood boron level was $1.64 \mathrm{mg} / 100 \mathrm{~mL}$ (Gordon et al. 1973).

Existing animal studies do not provide evidence that the nervous system is a toxicity target of repeated oral exposure to boric acid or borates, but no studies have examined batteries of neurological end points in animals following exposure to boron compounds. Relative brain weights were increased in mongrel dogs fed $60.5 \mathrm{mg}$ boron/kg/day as borax, but not boric acid, for 90 days and in rats fed 170 or $81 \mathrm{mg}$ boron/kg/day as borax or boric acid for 90 days or 2 years, respectively (Weir and Fisher 1972). No histological lesions were observed in these animals or in the brain or spinal cord of mice fed 577 or $201 \mathrm{mg}$ boron/kg/day as boric acid for 13 weeks or 2 years, respectively (NTP 1987; Dieter 1994).

In Wistar rats, exposure to approximately $20.8 \mathrm{mg}$ boron/ $\mathrm{kg} /$ day as borax (based on weight of $0.35 \mathrm{~kg}$ and average water consumption of $20.7 \mathrm{~mL}$ ) in drinking water for up to 14 weeks caused increased cerebral succinate dehydrogenase activity after 10 and 14 weeks of exposure (Settimi et al. 1982). Increased ribonucleic acid (RNA) concentration and increased acid proteinase activity in brain occurred after 14 weeks (Settimi et al. 1982). The neurological significance of these biochemical changes is unclear.

All LOAEL values for neurological effects in humans and animals are recorded in Table 3-2 and plotted in Figure 3-2.

\subsubsection{Reproductive Effects}

A survey of Turkish subpopulations compared fertility rates of 1,068 families living in two Turkish villages having drinking water boron levels of 2-29 mg/liter (from nearby geological deposits of calcium borate) with 610 families living in three other villages having drinking water boron levels of 0.03$0.4 \mathrm{mg} /$ liter (Sayli 1998a, 1998b; Sayli et al. 1998). Assuming 70-kg body weights and 2-L/day drinking water consumption, these boron levels would results in an estimated range of daily doses of 0.06$0.8 \mathrm{mg} / \mathrm{kg} /$ day. Three generations of families were represented. No significant differences in frequencies of infertility were observed between high- (2.34\% infertility) and low-exposure (2.62\% infertility) village groups. A separate analysis of the same subpopulation found no association of higher drinking water borate concentrations with increased rates of spontaneous abortions, stillbirths, or infant death (Tuccar et al. 1998). A follow-up study of this population reported no significant differences in infertility frequencies between the two populations (Sayli 2003). 
A questionnaire was administered to 542 male workers at a borax and boric acid production facility in California inquiring about the ability to conceive a child after at least 9 months of employment at the facility (Whorton et al. 1994). The worker population was categorized as having low, mid, or high exposures to borax or boric acid (exposure levels were not reported). A standardized birth ratio (SBR) was calculated as the number of children born to wives compared to the number of births expected in the same fraction of the U.S. population. The calculated SBR of 113 indicated higher birth rates among the borate workers relative to the U.S. population. Thus, this survey provided no evidence for association between occupational exposure to borax or boric acid and impaired fertility; however, the study is limited by non-rigorous survey design, lack of quantitative exposure data, and lack of a comparable comparison (control) group.

A cross-sectional survey of 1,187 Chinese boron mining and processing workers examined the association of boron exposure and various lifestyle factors to multiple reproductive indices (Chang et al. 2006). No exposure estimates were reported, but boron levels in drinking water and staple foodstuffs were significantly higher for surveyed workers compared to a comparison population. After correcting for age, race, diet, alcohol consumption, smoking, and education, there were no statistically significant differences between workers and the comparison population for ability to sire offspring, delayed pregnancy, multiple births, miscarriages, induced abortions, stillbirths, or tubal or ectopic pregnancies.

Liu et al. reported two studies of 176 (2006a) and 195 (2006b) Chinese men exposed to between 13 and $430 \mathrm{mg}$ boron/day. The investigators found no significant difference in semen quality and sperm motility, speed, or departure from a straight-line path in groups of borate workers, non-occupationally exposed men from a nearby community with high environmental levels of borates, and men from a community with low environmental levels of borates. However, data for estimated boron exposures for each group were not available.

A study of 146 Chinese men comprising borate workers, a community group, and controls were assessed for chromosomal shifts in the ratio of $\mathrm{Y}: \mathrm{X}$ chromosomes in sperm (Robbins et al. 2008). A significant correlation was found for $\mathrm{Y}: \mathrm{X}$ ratios and internal boron doses in blood, semen, and urine. Estimated daily boron exposures of 41.2, 4.3, and $2.3 \mathrm{mg} /$ day in the borate workers, community group, and controls were associated with mean $\mathrm{Y}: \mathrm{X}$ sperm ratios of $0.93,0.96$, and 0.99, respectively. However, no dose-response relationship was observed in the reported percentage of men who had fathered "more boys than girls", which was 57.7, 42.3, and 76.7\% in the borate workers, community group, and controls, respectively. There were several possible confounding factors in this study, including (1) the exclusion of men who had 
fathered equal numbers of boys and girls, (2) the average number of children/subject was $<2$ (approximately 1.3), and (3) a high rate of elective abortions across groups (49.2, 28.2, and 50.0\% for borate workers, community group, and controls, respectively), in which some men may have favored raising male offspring while complying with China's one-child-only policy. Thus, it cannot be determined from these data if a boron-associated shift in the $Y: X$ sperm ratio resulted in a change in sex of offspring.

While the human survey database is extensive in that large populations with chronic exposure durations were sampled from multiple locations (Chang et al. 2006; Liu et al. 2006a, 2006b; Robbins et al. 2008; Sayli 1998a, 1998b, 2003; Sayli et al. 1998; Whorton et al. 1994), they are limited for informing dose response relationships for reproductive effects. Specifically, the reliance on questionnaires and lack of clinical observations, absence of an appropriate comparison population (Robbins et al. 2008; Whorton et al. 1994), or low confidence in estimates of personal or group boron exposure (Liu et al. 2006a, 2006b) preclude these data from providing a basis for deriving an oral MRL.

Animal studies of acute-, intermediate-, and chronic- oral exposure to boric acid or borax consistently identified testicular atrophy and histological lesions and the associated impacts on spermatogenesis as the most sensitive reproductive effect. The majority of studies were performed in rats; however, the effects observed in rats were also observed in mice and dogs. The effects of boron on animal testes appear to be dose- and duration-related.

Acute-duration (2-week) oral gavage or dietary exposures of Wistar rats to $88 \mathrm{mg}$ boron/kg/day as boric acid produced significant damage to male reproductive tissues, but doses of 53 or $44 \mathrm{mg}$ boron/kg/day were without effect (Fukuda et al. 2000; Ku et al. 1993a; Kudo et al. 2000). Exposure to 88 mg boron/kg/day resulted in 12 and 13\% reduction in absolute and relative testes weights, respectively, multinucleated giant cell formation, increased residual body-like structures in the testes, degeneration/necrosis of germ cells, increased cellular debris in the epididymal ducts, exfoliation of round spermatids, and mild inhibition of spermiation (retention of step 19 spermatids at stages IX-XI) (Fukuda et al. 2000; Kudo et al. 2000).

Intermediate-duration gavage studies in rats resulted in similar effects as observed in the acute studies, but at lower exposure levels. Wistar rats given gavage doses of $26 \mathrm{mg}$ boron/kg/day as boric acid for 3 weeks exhibited decreased sperm motility, morphologically-abnormal sperm heads and tails, and increased preimplantation fetal loss when treated males were mated with untreated females; no reproductive effects 
occurred in this study with exposure to $9 \mathrm{mg}$ boron/kg/day (Yoshizaki et al. 1999). Four-week daily gavage doses of $44 \mathrm{mg}$ boron/kg/day as boric acid resulted in exfoliation of testicular and epididymal germ cells in Wistar rats (Kudo et al. 2000). Fukuda et al. (2000) gave gavage doses of $53 \mathrm{mg}$ boron/kg/day to Wistar rats for 4 weeks and observed 13 and 15\% reductions in absolute and relative testes weights, respectively, cellular debris in the testes, caudal and caput epididymis, focal atrophy of the seminiferous tubules, and decreased number of sperm in the ducta lumina. Rats given 4-week daily gavage doses of $88 \mathrm{mg}$ boron/kg/day group exhibited reduced sperm motility, reduced total sperm in caudal epididymis, atrophy of seminiferous tubules, atypical residual bodies, multinucleated giant cell formation, and the inability to impregnate females (Yoshizaki et al. 1999).

Intermediate-duration feeding studies in rats reported effects similar to those of the gavage studies. Rats fed $100 \mathrm{mg}$ boron/kg/day as borax in the diet, but not $50 \mathrm{mg}$ boron/kg/day, for 30 and 60 days showed testicular atrophy (Lee et al. 1978). Sprague-Dawley rats fed $86 \mathrm{mg}$ boron/kg/day as borax in the diet for 30 or 60 days were infertile for 3 or 5 weeks after exposure, respectively (Dixon et al. 1979). Exposure to $43 \mathrm{mg}$ boron/kg/day as borax for 60 days produced reduced testicular and epididymal weights and diameter of seminiferous tubules occurred, and reduced testicular levels of hyaluronidase, sorbitol dehydrogenase, and lactic acid dehydrogenase (isoenzyme-X) at 30 days (Dixon et al. 1979). Mildly inhibited spermiation was observed in Fischer 344 rats exposed to $26 \mathrm{mg}$ boron/kg/day in the diet for 59 weeks (Ku et al. 1993a). Fischer 344 rats fed $61 \mathrm{mg}$ boron/kg/day as boric acid for 4 weeks showed inhibited spermiation, appearance of peripheral spermatid nuclei, and spermatocyte sloughing/epithelial disorganization (Treinen and Chapin 1991), while severe inhibition of spermiation and testicular atrophy were observed in Fischer 344 rats exposed to 68 or $52 \mathrm{mg}$ boron/kg/day as boric acid in the diet for 6 or 9 weeks (Ku et al. 1993a). Full recovery from inhibition of spermiation was observed in a $38 \mathrm{mg}$ boron/kg/day group by 16 weeks after cessation of exposure for 9 weeks, but no recovery from testicular atrophy was observed in the 52 and $68 \mathrm{mg}$ boron/kg/day groups up to 32 weeks after exposure ended (Ku et al. 1993a). Dose-related elevations of FSH and LH suggested that boron exposure did not affect the compensatory response to atrophy (Ku et al. 1993a). In a recent study, Sprague-Dawley rats fed $136 \mathrm{mg}$ boron/kg/day as boric acid for 60 days exhibited decreased weights of testes, epididymes, seminal vesicles, prostate, and vas deferens; decreased sperm motility, spermatocyte, spermatid, and Leydig cell numbers; decreased testosterone levels, sexually aggressive behavior, sexual mounts, number of females impregnated, and viable pups/impregnated female; and increased cellular degeneration, ejaculation time, postejaculatory interval, and fetal resorptions in impregnated females (Nusier and Bataineh 2005). 
In intermediate-duration drinking water studies in rats, no reproductive effects (reproductive organ weight or histolopathology) were evident in Sprague-Dawley rats following exposure to $0.6 \mathrm{mg}$ boron $/ \mathrm{kg} / \mathrm{day}$ as borax for 90 days (Dixon et al. 1976). In another study, impaired spermatogenesis was observed in LongEvans rats given $44.7 \mathrm{mg}$ boron/kg/day as borax in drinking water for 70 days (Seal and Weeth 1980). Complete sterility was observed in Sprague-Dawley rats fed 1,170 ppm boron equivalents in the diet as boric acid or borax (at an estimated dose level of 101 and $116 \mathrm{mg}$ boron /kg/day for males and females); sterility was associated with a lack of viable sperm in atrophied testes in males and decreased ovulation in females (Weir and Fisher 1972). Rats were exposed for 14 weeks before mating in this study. No pregnancies occurred when female rats exposed to this dose level were mated with non-exposed male rats. At lower exposure levels (10 or $30 \mathrm{mg}$ boron/kg/day for males and 12 or $35 \mathrm{mg}$ boron/kg/day for females), no exposure-related adverse effects were found on overall fertility indices in three successive generations (Weir and Fisher 1972).

Studies in mice and dogs support the observations of reproductive effects seen in rats. In a study in which male CD-1 mice were exposed to gavage doses of boric acid of $0,21,70$, or $210 \mathrm{mg}$ boron/kg/day for 21 days ( 5 days before mating, during 5 days of mating, and extending to 21 total days of exposure), average testes weights were decreased at doses $\geq 70 \mathrm{mg}$ boron/kg/day, and exfoliation/disruption of seminiferous tubules and inhibited spermiation were observed at $210 \mathrm{mg}$ boron/ $\mathrm{kg} /$ day (Harris et al. 1992). Exposed male mice were mated to similarly exposed female mice (except that females were exposed for 8 days before mating), but no exposure-related effects were found on the percentage of females who became pregnant, the number of live pups per litter, or the weight of pups at birth (Harris et al. 1992). Degeneration of the seminiferous tubules was seen in mice exposed to $288 \mathrm{mg}$ boron/ $\mathrm{kg} / \mathrm{day}$ as boric acid in the diet for 13 weeks, while 103-week dietary exposure to $201 \mathrm{mg}$ boron/ $\mathrm{kg} / \mathrm{day}$ as boric acid resulted in testicular atrophy, degeneration of the seminiferous tubules, and interstitial hyperplasia (Dieter 1994; NTP 1987).

In a 2-generation (27-week) feeding study in CD-1 mice using a continuous breeding protocol, seminiferous tubule degeneration, impaired spermatogenesis, and reduced sperm motility resulted from $\geq 111 \mathrm{mg}$ boron/kg/day as boric acid (Fail et al. 1991). These doses were also associated with reduced litter size and fetal body weight. No effects were observed in a $27 \mathrm{mg}$ boron/kg/day dose group.

In mongrel dogs fed boric acid or borax for 90 days, severe testicular atrophy was seen at 60.5, but not at $6 \mathrm{mg}$ boron/kg/day (Weir and Fisher 1972). With 2 years of exposure, testicular atrophy and 
spermatogenic arrest were observed in dogs exposed to 22.8, but not in dogs exposed to $6.8 \mathrm{mg}$ boron/kg/day (Weir and Fisher 1972).

Effects of boric acid or borates on female reproductive organs and their functions are less clearly identified and studied in animals than effects on male reproductive organs. When pregnant CD-1 mice were exposed to gavage doses of $210 \mathrm{mg}$ boron/kg/day on gestation days 8-14, all dams failed to deliver litters (Harris et al. 1992). Exposure to 21 or $70 \mathrm{mg}$ boron/kg/day during the same period did not affect littering ability, average litter weight, or the number of live neonates at postnatal days 0,1 , and 4 (Harris et al. 1992). Mechanistic aspects of this effect of gestational exposure on littering capability of pregnant rats are unstudied. As discussed earlier, female Sprague Dawley rats exposed for 14 weeks to 1,170 ppm boron equivalents in the diet as boric acid or borax (estimated dose level of $116 \mathrm{mg}$ boron $/ \mathrm{kg} / \mathrm{day}$ ) did not become pregnant when mated with non-exposed males (Weir and Fisher 1972). The female sterility response at this dose level was associated with decreased ovulation.

The highest NOAEL values and all reliable LOAEL values for reproductive effects in animals and duration category are recorded in Table 3-2 and plotted in Figure 3-2.

\subsubsection{Developmental Effects}

No studies were available identifying developmental toxicity in humans from exposure to boron. However, several types of developmental effects (e.g., decreased fetal body weight, increased incidence of skeletal abnormalities) in animals were observed in standard developmental toxicity studies involving oral exposure of pregnant mice, rats, and rabbits to boric acid of borate salts. In addition, reduced pup weight at birth has been observed in animals receiving intermediate-duration exposures.

In mice, reduced fetal body weight and skeletal malformations were seen following acute- and intermediate-duration maternal oral exposures to boric acid.

Pregnant CD-1 mice fed $79 \mathrm{mg}$ boron/kg/day as boric acid on gestation days 0-17 had fetuses with 33\% lower body weight compared with controls, while fetal skeletal effects (e.g., short rib XIII, agenesis of lumbar vertabra, fused ribs) were reported at $175.3 \mathrm{mg}$ boron $/ \mathrm{kg} /$ day on gestation days 0-17 (Heindel et al. 1992, 1994). No effects on fetal development were observed in the $43.4 \mathrm{mg}$ boron/kg/day dose group (Heindel et al. 1992, 1994). 
No effects on implantation sites, littering, number of live pups per litter, or postnatal pup weight were observed following gavage exposure of pregnant CD-1 mice to $70 \mathrm{mg}$ boron $/ \mathrm{kg} /$ day as boric acid on gestation days 8-14 (Harris et al. 1992).

In an acute-duration study, pregnant CD-1 mice were given gavage doses of boric acid on various gestation days to examine the influence of stage of fetal development on skeletal malformations caused by boric acid (Cherrington and Chernoff 2002). When two gavage doses of $70 \mathrm{mg}$ boron/kg as boric acid were given on gestation day $6,7,8,9$, or 10 , increased incidence of fetuses with cervical rib and rib agenesis were observed in the groups treated on gestation day 8, but not with exposure on gestation days 6, 7, 9, or 10 (Cherrington and Chernoff 2002). Similarly, increased incidences of fetuses with cervical rib, rib agenesis, reduced rib length, and fused ribs were seen after twice daily doses of $70 \mathrm{mg}$ boron/kg on gestation days 6-8. Reduction in length of fetal rib XIII was seen in groups dosed once daily with $88 \mathrm{mg}$ boron/kg/day on gestation days 6-10. A single dose of $131 \mathrm{mg}$ boron/kg on gestation day 8 resulted in increased incidence of fetuses with cervical ossification, while two doses of $131 \mathrm{mg}$ boron/kg on gestation day 8 caused multiple thoracic skeletal malformations. Reduced fetal weight was observed in all treated groups (Cherrington and Chernoff 2002). This study did not identify acute NOAELs for fetal skeletal effects in mice. The study authors suggested that boric acid may alter gastrulation and presomitic mesoderm formation in CD-1 mice, which are key gestational milestones for axial skeletal development.

In rats, acute-duration developmental toxicity data were not available. However, intermediate-duration oral exposure of pregnant rats exhibited effects on fetal skeletal development and fetal weight, similar to those observed in mice. Pregnant Sprague-Dawley rats fed 13.6 boron $/ \mathrm{kg} / \mathrm{day}$ as boric acid in the diet on gestation days 0-20 had reduced fetal body weight, and increased incidence of fetuses with skeletal abnormalities at gestation day 20 occurred in groups of dams fed $28.4 \mathrm{mg}$ boron/kg/day (Heindel et al. 1992). Skeletal abnormalities observed in groups fed $28.4 \mathrm{mg}$ boron $/ \mathrm{kg} /$ day included agenesis or shortening of rib XIII, increased incidence of fetuses with enlargement of the lateral ventricles of the fetal brain, and increased resorptions (Heindel et al. 1992). Pregnant Sprague-Dawley rats fed $10 \mathrm{mg}$ boron/kg/day as boric acid on gestation days 0-20 exhibited no developmental effects, but exposures of $13 \mathrm{mg}$ boron/kg/day as boric acid resulted in decreased fetal body weight and skeletal abnormalities seen on gestation day 0 . However, in a second phase of this study, identically treated dams were allowed to litter and pups were observed through postnatal day 21. Upon necropsy, these pups did not exhibit significantly different body weights or incidences of skeletal abnormalities seen and fetuses examined on gestation day 0 (Price et al. 1996a, 1998). 
Developmental toxicity data in rabbits are available only for acute-duration oral exposures (Price et al. 1996b). Pregnant New Zealand white rabbits given gavage doses of $44 \mathrm{mg}$ boron/kg/day as boric acid on gestation days 6-19 exhibited increased maternal body weight (corrected for gestation) and reduced maternal kidney weight, gravid uterine weight, fetal body weight, number of ovarian corpora lutea, number of implantation sites, and live fetuses, compared with controls. Resorptions and fetal external (cleft palate), visceral (enlarged lateral ventricle of the brain), skeletal (cleft sternum, fused sternebrae), and cardiovascular (enlarged aorta, interventricular septal defect) malformations were increased, compared with controls. No significant maternal or fetal effects were observed following gavage doses of $22 \mathrm{mg}$ boron/kg/day as boric acid. The observed effects are consistent with those seen in acute-, intermediate-, and chronic-duration oral exposures in other animals. These data represent the most sensitive adverse effects observed in any species following acute-duration oral exposures. Thus, an acuteduration MRL of $0.2 \mathrm{mg}$ boron/ $\mathrm{kg} /$ day was derived based on a NOAEL of $22 \mathrm{mg}$ boron/ $\mathrm{kg} /$ day and a LOAEL of $44 \mathrm{mg}$ boron/kg/day for developmental effects in New Zealand white rabbits (Price et al. 1996b).

With intermediate-duration oral exposure to boric acid, reduced fetal body weight and skeletal abnormalities were consistently observed in developmental toxicity assays of mice, rats, and rabbits. Skeletal malformations increased in variety and severity with dose. However, reductions in fetal body weight appear to occur at lower exposure levels than those associated with skeletal abnormalities. With intermediate-duration exposure during gestation, the most sensitive developmental effect identified across all three species was reduced fetal weight in pregnant Sprague-Dawley rats fed $13.6 \mathrm{mg}$ boron/ $\mathrm{kg} / \mathrm{day}$ in the diet on gestation days $0-20$. This effect was seen at lower intermediate-duration exposure levels than the lowest intermediate-duration oral dose associated with reproductive effects in rats (i.e., a LOAEL of $26 \mathrm{mg}$ boron/kg/day was identified for inhibition of spermiation in rats) (Ku et al. 1993a). An intermediate oral MRL of $0.2 \mathrm{mg}$ boron/ $\mathrm{kg} /$ day was calculated as described in the footnote on Table 3-2, based on a benchmark dose analysis (Allen et al. 1996) of combined data sets (Heindel et al. 1992; Price et al. 1996a) for reduced fetal body weight in rats.

The highest NOAEL values and all reliable LOAEL values for developmental effects in animals and duration category are recorded in Table 3-2 and plotted in Figure 3-2. 


\subsubsection{Cancer}

Three epidemiological studies have associated increased boron intake in drinking water with decreased incidences of prostate and vaginal cancer. Cui et al. (2004) used the cross-sectional data from the NHANES III study, conducted from 1988 to 1994, which contained health and diet information for the non-institutionalized U.S. population. These investigators reported that men with mean intakes of $\geq 1.54 \mathrm{mg}$ boron/day had significantly less risk of developing prostate cancer than men ingesting $\leq 0.52 \mathrm{mg} /$ day. This study was limited by its cross-sectional design and reliance on 1-day recall of diet information to estimate boron exposure. A second study (Barranco et al. 2007) on a Texas population correlated increased boron in groundwater with reduced prostate cancer incidence rates. However, the observed correlation appeared to be driven primarily by 2-3 specific cases. Korkmaz et al. (2007) studied 1,059 rural Turkish women and associated higher boron intake (as evidenced by approximately 8-fold higher urinary boron concentration) with lower incidences of cervical cytopathology (0 findings in the high-boron group, 15 cases in the low-boron group). While this study did attempt to correct for lifestyle factors and other genotoxic confounders, it was cross-sectional in design. The hypotheses drawn from these studies are interesting; however, no clinical studies in humans or animals are available to substantiate effects of anti-tumor protection offered by boron.

No evidence of exposure-related cancer was observed in rats exposed to $81 \mathrm{mg}$ boron/kg/day as boric acid or borax for 2 years (Weir and Fisher 1972), dogs exposed to $6.8 \mathrm{mg}$ boron $/ \mathrm{kg} /$ day as boric acid or borax for 2 years (Weir and Fisher 1972), or mice exposed to $201 \mathrm{mg}$ boron/kg/day as boric acid for 2 years (Dieter 1994; NTP 1987). In nude mice subcutaneously injected with human LNCaP cells (prostate tumor clones), oral gavage doses of boric acid were given for 8 weeks (Gallardo-Williams et al. 2004) to determine if boron offered protection against prostate tumor growth. Although there was no significant difference between control and boron-treated mice in tumor incidences, the tumor sizes in mice given $1.7 \mathrm{mg}$ boron/kg/day were significantly smaller and the serum level of tumor specific antigen (PSA) was significantly less than controls.

\subsubsection{Dermal Exposure}

Information on dermal toxicity in humans involves exposure to the borates (as boric acid or borax), while the animal data involves exposure to boron oxide, which easily converts to boric acid in humid air or upon entering the mucosal layer of tissues. 


\subsubsection{Death}

No studies were located regarding death in humans or animals after dermal exposure to boron.

\subsubsection{Systemic Effects}

No studies were located regarding hematological and dermal/ocular effects in humans or regarding respiratory, cardiovascular, gastrointestinal, musculoskeletal, hepatic, or renal effects in humans or animals after dermal exposure to boron.

Hematological Effects. Draize and Kelley (1959) reported the application of 25-200 mg/kg/day boric acid in aqueous solution did not produce hematological changes when rubbed onto intact skin during a 90-day rabbit study. No quantitative data were provided; therefore, these results could not be evaluated.

Dermal Effects. Human data are limited to case reports of accidental exposure of the head. Three male workers (59-year-old waste handler and 34- and 36-year-old automotive mechanics) presented with general or focal alopecia of the scalp, presumably from spillage or wiping of boric acid or borax, respectively, onto the head (Beckett et al. 2001). In the case of the waste handler, the concentration of boric acid in the milieu of other known solvents in the waste tank was unknown. In the cases of the automotive workers, exposure was determined to arise from under-the-chassis flushing of automobile radiators which contained coolant solutions of ethylene glycol and 1-5\% borax. Actual exposures could not be determined. Blood sample analysis revealed no elevated blood boron levels in any of the subjects. Gradual and full hair re-growth occurred.

In animals, application of $1 \mathrm{~g}$ boron oxide dust to a $25 \mathrm{~cm}^{2}$ area of the skin of four rabbits produced erythema that lasted for 2-3 days (Wilding et al. 1959).

Ocular Effects. Instillation of boron oxide dust (50 mg) into the eyes of four rabbits produced conjunctivitis (Wilding et al. 1959). 
No studies were located regarding the following effects in humans or animals after dermal exposure to boron:

\subsubsection{Immunological and Lymphoreticular Effects}

\subsubsection{Neurological Effects}

\subsubsection{Reproductive Effects}

\subsubsection{Developmental Effects}

\subsubsection{Cancer}

\subsubsection{Other Routes of Exposure}

Direct application of a solution containing $6.3 \mathrm{mg}$ boron (as sodium perborate monohydrate) onto the cornea of rabbits resulted in mild irritancy of the epithelium and superficial stroma (Maurer et al. 2001).

\subsection{GENOTOXICITY}

No studies were located regarding genotoxic effects of boron by inhalation, oral, or dermal exposure in humans.

Results were predominantly negative in bacterial assays and in in vitro (Table 3-3) mammalian assays, including tests for chromosomal aberrations, gene mutation (Benson et al. 1984; Demerec et al. 1951; Haworth et al. 1983; Landolph 1985; NTP 1987), and cell transformation (Landolph 1985). Induction of $\beta$-galactosidase as part of the SOS response was observed in Escherichia coli PQ37, both with and without S-9 metabolic activation (Odunola 1997). Structural chromosomal aberrations, but not sister chromatid exchanges, were observed in cultured human lymphocytes exposed to boric acid (Arslan et al. 2008). 
Table 3-3. Genotoxicity of Boron In Vitro

\begin{tabular}{|c|c|c|c|c|}
\hline \multirow[b]{2}{*}{ Species (test system) } & \multirow[b]{2}{*}{ End point } & \multicolumn{2}{|c|}{ Results } & \multirow[b]{2}{*}{ Reference } \\
\hline & & $\begin{array}{l}\text { With } \\
\text { activation }\end{array}$ & $\begin{array}{l}\text { Without } \\
\text { activation }\end{array}$ & \\
\hline \multicolumn{5}{|l|}{ Prokaryotic organisms: } \\
\hline \multirow[t]{3}{*}{ Salmonella typhimurium } & Gene mutation & - & - & Haworth et al. 1983 \\
\hline & Gene mutation & - & - & Benson et al. 1984 \\
\hline & Gene mutation & - & - & NTP 1987 \\
\hline Escherichia coli & Gene mutation & - & - & Demerec et al. 1951 \\
\hline \multicolumn{5}{|l|}{ Mammalian cells: } \\
\hline Mouse lymphoma & Gene mutation & - & - & NTP 1987 \\
\hline Mouse embryo fibroblast & Gene mutation & NA & - & Landolph 1985 \\
\hline Human foreskin fibroblast & Gene mutation & NA & - & Landolph 1985 \\
\hline Chinese hamster ovary & Gene mutation & NA & - & Landolph 1985 \\
\hline Chinese hamster ovary & $\begin{array}{l}\text { Chromosomal } \\
\text { aberration }\end{array}$ & - & - & NTP 1987 \\
\hline Human lymphocytes & $\begin{array}{l}\text { Sister chromatid } \\
\text { exchange }\end{array}$ & NA & - & Arslan et al. 2008 \\
\hline Human lymphocytes & $\begin{array}{l}\text { Chromosomal } \\
\text { aberrations }\end{array}$ & NA & + & Arslan et al. 2008 \\
\hline
\end{tabular}

$-=$ negative result; $\mathrm{NA}=$ not applicable 


\subsection{TOXICOKINETICS}

\subsubsection{Absorption}

\subsubsection{Inhalation Exposure}

Reports of upper respiratory tract symptoms of irritation following exposure to boron oxide and boric acid dusts suggest that boron can deposit in the upper airway (Garabrant et al. 1984, 1985). Borax production workers were found to have approximately an order of magnitude higher blood and urine concentrations of boron at the end of a work shift compared to the beginning, suggesting that inhaled boron is absorbed and systemically distributed (Culver et al. 1994a).

\subsubsection{Oral Exposure}

Near-complete gastrointestinal absorption was indicated in humans as evidenced by the urinary recovery of 93.9\% of the ingested dose of boric acid over a 96-hour collection period (Jansen et al. 1984a). Dourson et al. (1998) reviewed data from the literature to estimate oral absorption fractions of 81-92\% for humans and 95\% for animals (rats).

\subsubsection{Dermal Exposure}

Volunteers exhibited 0.23 and $0.21 \%$ percutaneous absorption of $1.8 \mathrm{~mL}$ of $5 \%$ solutions of boric acid and borax (Wester et al. 1998). Urinary excretion studies in humans (Section 3.4.4.3) suggest there is very little absorption of boron through intact skin. Excretion studies (Section 3.4.4.3) in rabbits suggest that boron is readily absorbed following contact with damaged skin (Draize and Kelley 1959).

\subsubsection{Distribution}

No quantitative studies were located regarding distribution in humans.

\subsubsection{Inhalation Exposure}

No studies were located regarding distribution of boron in animals after inhalation exposure. 


\subsubsection{Oral Exposure}

Boron evenly distributed to liver, kidney, brain, muscle, adrenals, epididymis, testes, seminal vesicles, and blood, but not fat, of male rats fed $61 \mathrm{mg}$ boron/kg/day as boric acid (9,000 ppm) for 1-28 days (Ku et al. 1991; Moseman 1994; Treinen and Chapin 1991), reaching steady-state by 4 days. Blood and testes boron levels were similar in rats fed 26-68 mg boron/kg/day as boric acid (3,000-9,000) for 9 weeks $(\mathrm{Ku}$ et al. 1991). However, boron accumulated in bone in male rats fed $61 \mathrm{mg}$ boron/ $\mathrm{kg} /$ day (as boric acid) for 9 weeks, with achievement of steady-state at 4 weeks. Bone levels were approximately 3 -fold higher than soft tissue levels (Moseman 1994).

\subsubsection{Dermal Exposure}

No studies were located regarding distribution of boron in animals after dermal exposure.

\subsubsection{Metabolism}

As an inorganic chemical, boron is not expected to be metabolized by humans or animals. Studies of inhalation and oral exposure of animals and humans to borates have consistently reported recovery of the parent borate only in the blood, tissues, and urine (Culver et al. 1994a; Draize and Kelley 1959; Jansen et al. 1984a; Ku et al. 1991; Moseman 1994; Treinen and Chapin 1991).

\subsubsection{Elimination and Excretion}

\subsubsection{Inhalation Exposure}

Over 94\% of estimated total boric acid (mean $11.84 \mathrm{mg}$ boron/day) inhaled and ingested by Chinese borate workers was eliminated 24 hours later in the urine (Xing et al. 2005) as determined by the percent estimated daily dose recovered in the urine. In rats that inhaled average concentrations of $77 \mathrm{mg} / \mathrm{m}^{3}$ boron oxide aerosols over a 22-week period, an average of $11.90 \mathrm{mg}$ boron/kg/day was detected in the urine compared to $0.24 \mathrm{mg} / \mathrm{kg} /$ day in untreated control groups (Wilding et al. 1959).

\subsubsection{Oral Exposure}

Over 93\% of the administered dose was excreted in the urine of six male volunteers 96 hours after administration of a single oral dose of $1.9 \mathrm{mg}$ boron $/ \mathrm{kg}$ (as boric acid) (Jansen et al. 1984a). An analysis of nine cases involving boric acid poisoning revealed a mean half-life of 13.4 hours (range 4-27.8 hours). 
There was no correlation between half-life and calculated serum boric acid level at $t_{0}(r=0.08, p=0.84)$ (Litovitz et al. 1988). Boric acid was detected in urine of patients 23 days after a single ingestion (Wong et al. 1964). Renal clearance of dietary boron from fifteen pregnant women was calculated to be $1.02 \mathrm{~mL} / \mathrm{minute} / \mathrm{kg}$, or $66.1 \mathrm{~mL} /$ minute (Pahl et al. 2001).

In rabbits, $50-66 \%$ of the administered dose was recovered in urine after ingestion of 17.1-119.9 mg boron/kg/day as boric acid (Draize and Kelley 1959). In rats fed 26-68 mg boron/kg/day as boric acid (3,000-6,000 ppm) for 9 weeks, boron concentrations in bone began decreasing after cessation of exposure; however, bone levels remained approximately 3-fold higher than controls for up to 32 weeks (Chapin et al. 1997; Moseman 1994). Blood levels in these same animals returned to control levels within 7 days of exposure cessation (Ku et al. 1991). Using literature data, Dourson et al. (1998) estimated the fraction eliminated of absorbed boron to be $67-98 \%$ in humans and $99 \%$ in rats. These investigators also calculated clearance values of $40 \mathrm{mg} / \mathrm{kg} / \mathrm{hour}$ in humans, $163 \mathrm{mg} / \mathrm{kg} / \mathrm{hour}$ in rats, and $397 \mathrm{mg} / \mathrm{kg} / \mathrm{hour}$ in pregnant rats. Pregnancy did not affect renal clearance $(0.2 \mathrm{~L} / \mathrm{hour} / \mathrm{kg}$ or $1.0 \mathrm{~mL} / \mathrm{minute}$ ) or elimination half-life (3.2 hours) in rats given gavage doses of $0.05-5 \mathrm{mg}$ boron/kg/day (as boric acid) on gestation day 16 (Vaziri et al. 2001).

\subsubsection{Dermal Exposure}

Limited data in humans suggest that very little absorption of boron occurs through intact skin. There was no increase in the urinary excretion of boron in one human subject following the application of $15 \mathrm{~g}$ boric acid (37.5 mg boron/kg body weight) on the forearm for 4 hours (Draize and Kelley 1959). In volunteers having $1.8 \mathrm{~mL}$ of $5 \%$ boric acid or borax solutions applied to the back and left for 24 hours, urinary boron levels increased to peak values of $<0.1 \%$ above background levels at 14 days following exposure (Wester et al. 1998).

Animal studies support human findings. Draize and Kelley (1959) applied $200 \mathrm{mg} / \mathrm{kg}$ as boric acid to intact, abraded or burnt, and partially denuded skin of rabbits. Net urinary excretion of boric acid per 24 hours during 4 consecutive days of compound treatment was $1.4,7.6$, and $21.4 \mathrm{mg} / \mathrm{kg}$, respectively (0.25, 1.3, and $3.7 \mathrm{mg}$ boron/kg, respectively).

\subsubsection{Other Routes of Exposure}

In eight adult volunteers administered a single dose of boric acid (562-611 mg) by intravenous infusion, 98.7\% of the administered dose was recovered in urine 120 hours after injection (Jansen et al. 1984b). 
Renal blood clearance averaged $39.1 \mathrm{~mL} /$ minute per $1.73 \mathrm{~m}^{2}$ surface area in eight adult human subjects administered intravenous injections of $35 \mathrm{mg}$ boron $/ \mathrm{kg}$ (as sodium pentaborate). Urine boron concentrations on the day of administration averaged $1.19 \mathrm{mg} / \mathrm{mL}$ (Farr and Konikowski 1963). In rats administered an intravenous infusion of $86 \mathrm{mg}$ boron/kg (as borax) (Tagawa et al. 2000), boron distributed rapidly to the extravascular tissues, giving a steady state volume of distribution of $1.19 \mathrm{~L} / \mathrm{kg}$. Excretion of boron was rapid, with $87.6 \%$ eliminated in the urine by 2 hours after infusion.

Pharmacokinetic analysis of the blood time course data resulted in an estimated elimination rate constant $\left(\mathrm{K}_{\mathrm{el}}\right)$ of 0.15 hour $^{-1}$ and a clearance rate of $0.11 \mathrm{~L} / \mathrm{hour} / \mathrm{kg}$. The elimination half-life was 8.43 hours. Guinea pigs given intra-tympanic doses of $0.2 \mathrm{~mL} /$ day of $4 \%$ boric acid in saline did not exhibit changes in hearing level (Oztukcan et al. 2008).

\subsubsection{Physiologically Based Pharmacokinetic (PBPK)/Pharmacodynamic (PD) Models}

Physiologically based pharmacokinetic (PBPK) models use mathematical descriptions of the uptake and disposition of chemical substances to quantitatively describe the relationships among critical biological processes (Krishnan et al. 1994). PBPK models are also called biologically based tissue dosimetry models. PBPK models are increasingly used in risk assessments, primarily to predict the concentration of potentially toxic moieties of a chemical that will be delivered to any given target tissue following various combinations of route, dose level, and test species (Clewell and Andersen 1985). Physiologically based pharmacodynamic (PBPD) models use mathematical descriptions of the dose-response function to quantitatively describe the relationship between target tissue dose and toxic end points.

PBPK/PD models refine our understanding of complex quantitative dose behaviors by helping to delineate and characterize the relationships between: (1) the external/exposure concentration and target tissue dose of the toxic moiety, and (2) the target tissue dose and observed responses (Andersen and Krishnan 1994; Andersen et al. 1987). These models are biologically and mechanistically based and can be used to extrapolate the pharmacokinetic behavior of chemical substances from high to low dose, from route to route, between species, and between subpopulations within a species. The biological basis of PBPK models results in more meaningful extrapolations than those generated with the more conventional use of uncertainty factors.

The PBPK model for a chemical substance is developed in four interconnected steps: (1) model representation, (2) model parameterization, (3) model simulation, and (4) model validation (Krishnan and Andersen 1994). In the early 1990s, validated PBPK models were developed for a number of 
toxicologically important chemical substances, both volatile and nonvolatile (Krishnan and Andersen 1994; Leung 1993). PBPK models for a particular substance require estimates of the chemical substancespecific physicochemical parameters, and species-specific physiological and biological parameters. The numerical estimates of these model parameters are incorporated within a set of differential and algebraic equations that describe the pharmacokinetic processes. Solving these differential and algebraic equations provides the predictions of tissue dose. Computers then provide process simulations based on these solutions.

The structure and mathematical expressions used in PBPK models significantly simplify the true complexities of biological systems. If the uptake and disposition of the chemical substance(s) are adequately described, however, this simplification is desirable because data are often unavailable for many biological processes. A simplified scheme reduces the magnitude of cumulative uncertainty. The adequacy of the model is, therefore, of great importance, and model validation is essential to the use of PBPK models in risk assessment.

PBPK models improve the pharmacokinetic extrapolations used in risk assessments that identify the maximal (i.e., the safe) levels for human exposure to chemical substances (Andersen and Krishnan 1994). PBPK models provide a scientifically sound means to predict the target tissue dose of chemicals in humans who are exposed to environmental levels (for example, levels that might occur at hazardous waste sites) based on the results of studies where doses were higher or were administered in different species. Figure 3-3 shows a conceptualized representation of a PBPK model.

If PBPK models for boron exist, the overall results and individual models are discussed in this section in terms of their use in risk assessment, tissue dosimetry, and dose, route, and species extrapolations.

There are no PBPK models available for boron. 


\section{Figure 3-3. Conceptual Representation of a Physiologically Based Pharmacokinetic (PBPK) Model for a Hypothetical Chemical Substance}

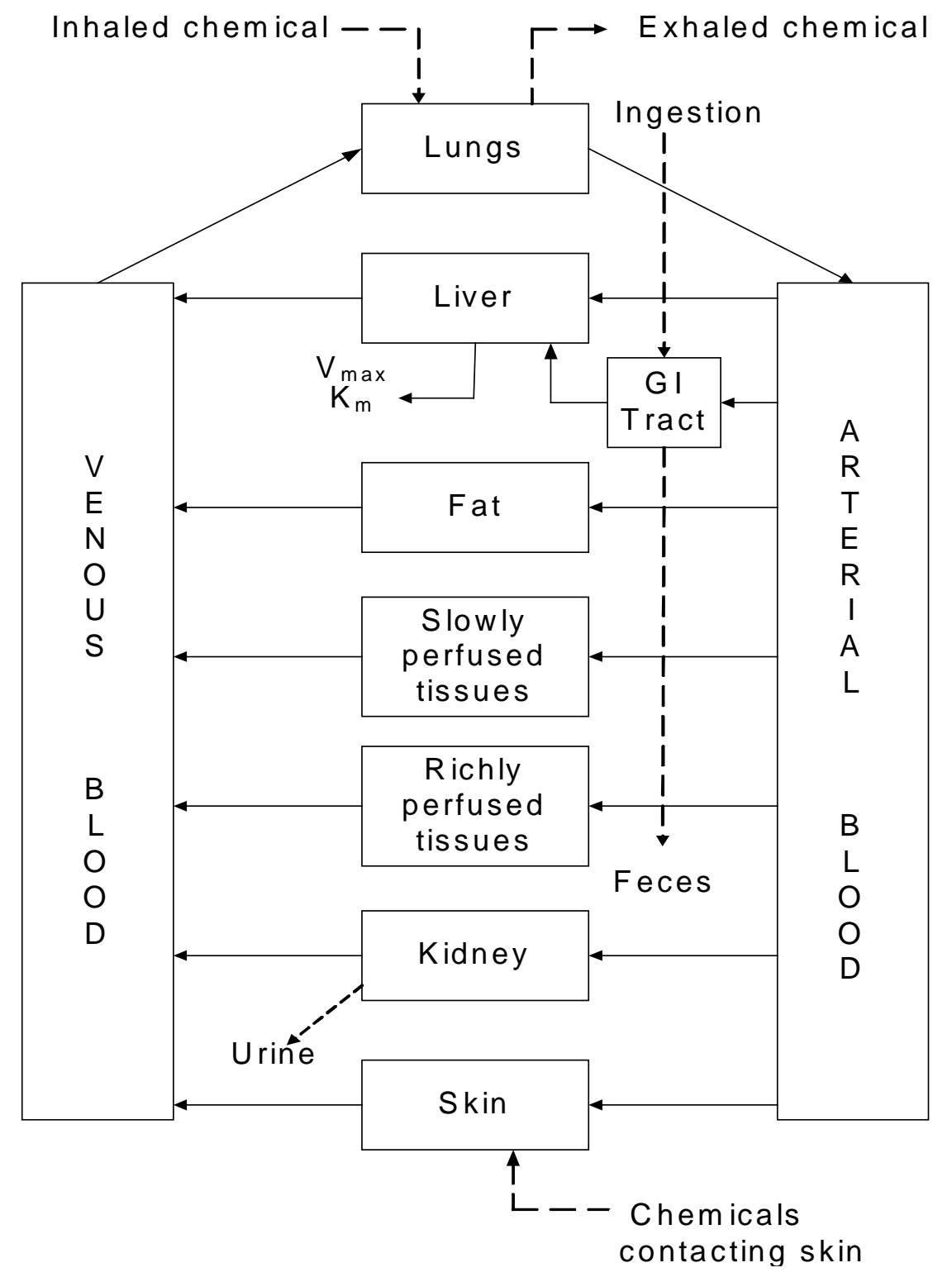

Note: This is a conceptual representation of a physiologically based pharmacokinetic (PBPK) model for a hypothetical chemical substance. The chemical substance is shown to be absorbed via the skin, by inhalation, or by ingestion, metabolized in the liver, and excreted in the urine or by exhalation.

Source: adapted from Krishnan and Andersen 1994 


\subsection{MECHANISMS OF ACTION}

\subsubsection{Pharmacokinetic Mechanisms}

Absorption. Boron is absorbed across pulmonary tissues into the blood, as seen in workers exposed to borate dusts, who were found to have higher blood and urine boron concentrations at the end of a work shift compared to the beginning of the shift (Culver et al. 1994a). Boron is almost completely absorbed in the gastrointestinal tract, with up to 92 and 95\% of ingested dose being recovered in the urine (Dourson et al. 1998). No data are available to indicate whether boron is actively transported or passively diffused across pulmonary or gastrointestinal tissues. Diet may influence the rate of boron absorption in the gut, as higher initial boron levels were found in the urine of humans given boron in an ointment vehicle, compared to administration via a water vehicle (Schou et al. 1984). Boron was found to be minimally absorbed across intact human or animal skin (Draize and Kelley 1959; Wester et al. 1998).

Distribution. Boron is distributed readily to all body tissues. Tissue levels from daily doses were observed to achieve steady-state with plasma in all tissues examined, including neurological and reproductive tissues, with the exception of bone and adipose tissues (Ku et al. 1991. Bone serves as a storage depot for boron, while adipose tissue has a lower affinity for boron than other soft tissues. The mechanism(s) of transport across tissue membranes and into bone are not known. No data were available identifying binding of boron to a carrier protein in the blood or plasma membranes.

Metabolism. Boron is a trace element and is not metabolized in the body. Borates exist in the body as boric acid, the only form of boron recovered in the urine.

Excretion. Excretion of systemically absorbed born is accomplished primarily through renal elimination, with minor fractions excreted in the saliva, sweat, and feces (Jansen et al. 1984a). No data are available regarding the contribution of tubular absorption of boron in the kidney. Glomerular filtration rate is likely the dominant factor in renal elimination of boron. As such, the systemic elimination of boron may be compromised in populations with reduced glomerular filtration rates (Dourson et al. 1998), such as preeclamptic women. This assumption is used in deriving chemical-specific uncertainty factors, which themselves are used for deriving the intermediate-duration oral MRL (Appendix A). 


\subsubsection{Mechanisms of Toxicity}

No studies were available in humans describing a mechanism of toxicity for neurological, gastrointestinal, hepatic, or renal effects observed in case reports of high-dose poisoning incidents. In animals, reproductive and developmental effects have been the most sensitive toxic end points observed.

Although several studies have examined possible mechanisms for reproductive toxicity, the actual toxic mechanism remains unknown. In rats, delayed spermiation (inhibited release of mature sperm) appears to be the hallmark event in testicular toxicity, followed by exfoliation of germ epithelium and atrophy at higher doses (Treinen and Chapin 1991). Leydig and Sertoli cell cultures exposed to $10 \mathrm{mM}$ boric acid did not exhibit reduced responsiveness to induction of testosterone production, but exhibited reduced intracellular cAMP levels following FSH stimulation (Ku et al. 1993b). Further, lactate and pyruvate (the primary energy sources for Sertoli cells) production (possibly from boronation of NAD cofactors [Ku et al. 1993b]) and deoxyribonucleic acid (DNA) synthesis were significantly reduced, suggesting that germ epithelial sloughing and testicular atrophy may result from impaired energy production and mitosis/meiosis in the Sertoli cells (Fail et al. 1998). However, in vivo delayed spermiation appears to occur at lower Sertoli cell exposure levels than disruption of energy production and DNA synthesis, making it difficult to conclude whether reproductive effects are hormonally or metabolically-mediated (Fail et al. 1998; Ku et al. 1993a).

The mechanism of toxicity for developmental effects is also unknown. Fail et al. (1998) suggest that the reduction in fetal body weight (the most sensitive end point observed in rats [Heindel et al. 1992]) may be due to mitotic inhibition observed in viruses, bacteria, insects, yeasts, and animals. Hyperacetylation of embryonic mouse tissues is highly associated with skeletal malformations following exposure to histone deacetylase inhibitors such as valproic acid and trichostatin A. Mice given intraperitoneal doses of $175 \mathrm{mg}$ boron/kg (as boric acid) on gestation day 8 exhibited hyperacetylation of embryonic somites, inhibition of histone deacetylase, and increased incidences of skeletal malformations (fused ribs and vertebra, changes in the typical number of axial segments in different axial districts). The association of these biochemical and morphological effects suggest that boric acid-induced skeletal malformations may result from inhibition of histone deacetylase. Wery et al. (2003) reported a cranial shift in the anterior limits of the hoxa6 and hoxc6 genes in the fetuses of pregnant rats given two gavage doses of $88 \mathrm{mg} / \mathrm{boron} / \mathrm{kg} /$ day (as boric acid) on gestation day 9. The control of position and development of the fetal vertebrae have been associated with the activity of these genes (see Section 3.3). It is not known whether inhibition of histone deacetylase, as suggested by Fail et al. (1998) is involved with the response of the hoxa6 and hoxc6 genes to in utero exposure to high doses of boron. 


\subsubsection{Animal-to-Human Extrapolations}

Similarities in rodents and humans for boron toxicokinetics and reproductive physiology suggest that the animal toxicity data are relevant for human risk assessment. Animal and rodents studies indicate that boron is readily absorbed (particularly via the oral route), not metabolized, and extensively eliminated via urinary excretion (Section 3.4). Male humans and rodents share similar physiological structures and hormonal control mechanisms of the reproductive system, suggesting a similar target of toxicity for reproductive effects. Similarly, the sequence of fetal developmental steps is similar between the species. Lack of human data, particularly reproductive and developmental data, for effects observed in animal studies introduce uncertainty into the extrapolation of animals data to humans.

\subsection{TOXICITIES MEDIATED THROUGH THE NEUROENDOCRINE AXIS}

Recently, attention has focused on the potential hazardous effects of certain chemicals on the endocrine system because of the ability of these chemicals to mimic or block endogenous hormones. Chemicals with this type of activity are most commonly referred to as endocrine disruptors. However, appropriate terminology to describe such effects remains controversial. The terminology endocrine disruptors, initially used by Thomas and Colborn (1992), was also used in 1996 when Congress mandated the EPA to develop a screening program for “...certain substances [which] may have an effect produced by a naturally occurring estrogen, or other such endocrine effect[s]...”. To meet this mandate, EPA convened a panel called the Endocrine Disruptors Screening and Testing Advisory Committee (EDSTAC), and in 1998, the EDSTAC completed its deliberations and made recommendations to EPA concerning endocrine disruptors. In 1999, the National Academy of Sciences released a report that referred to these same types of chemicals as hormonally active agents. The terminology endocrine modulators has also been used to convey the fact that effects caused by such chemicals may not necessarily be adverse. Many scientists agree that chemicals with the ability to disrupt or modulate the endocrine system are a potential threat to the health of humans, aquatic animals, and wildlife. However, others think that endocrine-active chemicals do not pose a significant health risk, particularly in view of the fact that hormone mimics exist in the natural environment. Examples of natural hormone mimics are the isoflavinoid phytoestrogens (Adlercreutz 1995; Livingston 1978; Mayr et al. 1992). These chemicals are derived from plants and are similar in structure and action to endogenous estrogen. Although the public health significance and descriptive terminology of substances capable of affecting the endocrine system remains controversial, scientists agree that these chemicals may affect the synthesis, secretion, transport, binding, action, or elimination of natural hormones in the body responsible for maintaining homeostasis, reproduction, 
development, and/or behavior (EPA 1997). Stated differently, such compounds may cause toxicities that are mediated through the neuroendocrine axis. As a result, these chemicals may play a role in altering, for example, metabolic, sexual, immune, and neurobehavioral function. Such chemicals are also thought to be involved in inducing breast, testicular, and prostate cancers, as well as endometriosis (Berger 1994; Giwercman et al. 1993; Hoel et al. 1992).

No studies were located regarding endocrine disruption in humans after exposure to boron.

While depressed testosterone blood levels was observed in rats fed diets with $61 \mathrm{mg}$ boron/kg/day as boric acid (Treinen and Chapin 1991), GnRH challenge in boron-fed rats (Fail et al. 1998) resulted in LH responses similar to controls and an exacerbated FSH response relative to controls, suggesting that peripheral hormonal changes were not due to neuroendocrine toxicity, but possibly to Leydig and Sertoli cell-specific effects (Fail et al. 1998).

No in vitro studies were located regarding endocrine disruption of boron.

\subsection{CHILDREN'S SUSCEPTIBILITY}

This section discusses potential health effects from exposures during the period from conception to maturity at 18 years of age in humans, when all biological systems will have fully developed. Potential effects on offspring resulting from exposures of parental germ cells are considered, as well as any indirect effects on the fetus and neonate resulting from maternal exposure during gestation and lactation. Relevant animal and in vitro models are also discussed.

Children are not small adults. They differ from adults in their exposures and may differ in their susceptibility to hazardous chemicals. Children's unique physiology and behavior can influence the extent of their exposure. Exposures of children are discussed in Section 6.6, Exposures of Children.

Children sometimes differ from adults in their susceptibility to hazardous chemicals, but whether there is a difference depends on the chemical (Guzelian et al. 1992; NRC 1993). Children may be more or less susceptible than adults to health effects, and the relationship may change with developmental age (Guzelian et al. 1992; NRC 1993). Vulnerability often depends on developmental stage. There are critical periods of structural and functional development during both prenatal and postnatal life, and a particular structure or function will be most sensitive to disruption during its critical period(s). Damage 
may not be evident until a later stage of development. There are often differences in pharmacokinetics and metabolism between children and adults. For example, absorption may be different in neonates because of the immaturity of their gastrointestinal tract and their larger skin surface area in proportion to body weight (Morselli et al. 1980; NRC 1993); the gastrointestinal absorption of lead is greatest in infants and young children (Ziegler et al. 1978). Distribution of xenobiotics may be different; for example, infants have a larger proportion of their bodies as extracellular water, and their brains and livers are proportionately larger than adults (Altman and Dittmer 1974; Fomon 1966; Fomon et al. 1982; Owen and Brozek 1966; Widdowson and Dickerson 1964). The infant also has an immature blood-brain barrier (Adinolfi 1985; Johanson 1980) and probably an immature blood-testis barrier (Setchell and Waites 1975). Many xenobiotic metabolizing enzymes have distinctive developmental patterns. At various stages of growth and development, levels of particular enzymes may be higher or lower than those of adults, and sometimes unique enzymes may exist at particular developmental stages (Komori et al. 1990; Leeder and Kearns 1997; NRC 1993; Vieira et al. 1996). Whether differences in xenobiotic metabolism make the child more or less susceptible also depends on whether the relevant enzymes are involved in activation of the parent compound to its toxic form or in detoxification. There may also be differences in excretion, particularly in newborns who all have a low glomerular filtration rate and have not developed efficient tubular secretion and resorption capacities (Altman and Dittmer 1974; NRC 1993; West et al. 1948). Children and adults may differ in their capacity to repair damage from chemical insults. Children also have a longer remaining lifetime in which to express damage from chemicals; this potential is particularly relevant to cancer.

Certain characteristics of the developing human may increase exposure or susceptibility, whereas others may decrease susceptibility to the same chemical. For example, although infants breathe more air per kilogram of body weight than adults breathe, this difference might be somewhat counterbalanced by their alveoli being less developed, which results in a disproportionately smaller surface area for alveolar absorption (NRC 1993).

Human data of boron toxicity in children and humans due to high-dose exposures are not adequate to identify the presence or lack of children's susceptibility. Normal boron blood levels in children and infants range from 0 to $1.25 \mu \mathrm{g} / \mathrm{mL}$ (Fisher and Freimuth 1958; O'Sullivan and Taylor 1983). Infants exhibiting adverse effects after ingestion of boric acid in infant formula had boron blood levels (reported as borate) of 20-150 $\mu \mathrm{g} / \mathrm{mL}$, with fatal cases having blood levels of 200-1,600 $\mu \mathrm{g} / \mathrm{mL}$ (Wong et al. 1964). Comparatively, adult serum boron level (as boric acid) of 2,320 $\mu \mathrm{g} / \mathrm{mL}$ was not associated with significant toxicity (Linden et al. 1986). 
No animal studies were located regarding susceptibility of immature animals to boron toxicity. However, oral exposure studies in pregnant animals have identified developmental effects in fetus exposed to boron (as borax or boric acid) in utero. No developmental effects were observed in animals following inhalation exposures. The observed effects from oral exposure include skeletal malformations and cardiovascular abnormalities. The most sensitive effect identified was reduced fetal body weight, which consistently occurred in animals at lower doses than required for skeletal malformations or other effects. The acute- and intermediate-duration oral MRLs are based on reduced fetal body weight in rats and should be protective of children developing in the womb.

\subsection{BIOMARKERS OF EXPOSURE AND EFFECT}

Biomarkers are broadly defined as indicators signaling events in biologic systems or samples. They have been classified as markers of exposure, markers of effect, and markers of susceptibility (NAS/NRC 1989).

A biomarker of exposure is a xenobiotic substance or its metabolite(s) or the product of an interaction between a xenobiotic agent and some target molecule(s) or cell(s) that is measured within a compartment of an organism (NAS/NRC 1989). The preferred biomarkers of exposure are generally the substance itself, substance-specific metabolites in readily obtainable body fluid(s), or excreta. However, several factors can confound the use and interpretation of biomarkers of exposure. The body burden of a substance may be the result of exposures from more than one source. The substance being measured may be a metabolite of another xenobiotic substance (e.g., high urinary levels of phenol can result from exposure to several different aromatic compounds). Depending on the properties of the substance (e.g., biologic half-life) and environmental conditions (e.g., duration and route of exposure), the substance and all of its metabolites may have left the body by the time samples can be taken. It may be difficult to identify individuals exposed to hazardous substances that are commonly found in body tissues and fluids (e.g., essential mineral nutrients such as copper, zinc, and selenium). Biomarkers of exposure to boron are discussed in Section 3.8.1.

Biomarkers of effect are defined as any measurable biochemical, physiologic, or other alteration within an organism that, depending on magnitude, can be recognized as an established or potential health impairment or disease (NAS/NRC 1989). This definition encompasses biochemical or cellular signals of tissue dysfunction (e.g., increased liver enzyme activity or pathologic changes in female genital epithelial 
cells), as well as physiologic signs of dysfunction such as increased blood pressure or decreased lung capacity. Note that these markers are not often substance specific. They also may not be directly adverse, but can indicate potential health impairment (e.g., DNA adducts). Biomarkers of effects caused by boron are discussed in Section 3.8.2.

A biomarker of susceptibility is an indicator of an inherent or acquired limitation of an organism's ability to respond to the challenge of exposure to a specific xenobiotic substance. It can be an intrinsic genetic or other characteristic or a preexisting disease that results in an increase in absorbed dose, a decrease in the biologically effective dose, or a target tissue response. If biomarkers of susceptibility exist, they are discussed in Section 3.10, Populations That Are Unusually Susceptible.

\subsubsection{Biomarkers Used to Identify or Quantify Exposure to Boron}

Boron in blood and urine can be used as an indicator of exposure to boron. Normal dietary concentrations of boron in the blood of humans range from 0 to $1.25 \mu \mathrm{g} / \mathrm{mL}$ in children and infants (Fisher and Freimuth 1958; O'Sullivan and Taylor 1983). Boron blood levels (reported as borate) of 20-150 $\mu \mathrm{g} / \mathrm{mL}$ have been associated with adverse systemic effects in infants who ingested boric acid in infant formula (Wong et al. 1964). Boron concentrations, expressed as borate, reported in fatal cases vary from 200 to $1,600 \mu \mathrm{g} / \mathrm{mL}$ in infants (Wong et al. 1964). In adults, a serum boron level (as boric acid) of 2,320 $\mu \mathrm{g} / \mathrm{mL}$ was not associated with significant toxicity (Linden et al. 1986).

Urinary excretion levels can also be useful indicators of elevated total body burden of boron. Concentrations of boron in the normal population range from 0.07 to $0.15 \mathrm{mg} / 100 \mathrm{~mL}$ (Vignec and Ellis 1954) and from 0.004 to $0.66 \mathrm{mg} / 100 \mathrm{~mL}$ (Imbus et al. 1963). In one infant, the urine contained $13.9 \mathrm{mg}$ boron/liter as borax or $1.38 \mathrm{mg}$ boron/mL of boric acid following ingestion of a borax and honey mixture over a period of 12 weeks (Gordon et al. 1973). Virtually complete urinary excretion was indicated by the recovery of $93.9 \%$ (over a 96-hour collection period) of a boric acid solution ingested by three volunteers (Jansen et al. 1984a).

Neurological, dermal, gastrointestinal, liver, and kidney effects in humans have been associated with exposure to boron. Studies in animals have demonstrated gonadal injury. Various clinical and biochemical tests exist that may provide useful information on exposure. However, similar effects are caused by a variety of other substances and are, therefore, not specific for boron exposure. 


\subsubsection{Biomarkers Used to Characterize Effects Caused by Boron}

Central nervous system injury, gastrointestinal effects, and skin damage are characteristic manifestations of boron toxicity in humans. Liver and kidneys in humans and testes in animals can also be affected. Various clinical and biochemical changes associated with these effects may be measured to detect the extent of exposure to boron. There is no single biological indicator of boron exposure; consequently, several parameters must be measured including boron levels in urine and blood and biochemical changes for systemic and neurological effects.

Neurological damage has been reported in humans. Neurological effects reported in humans have focused primarily on histopathological alterations. No data were provided on biochemical changes. In animals, testicular atrophy and reduced sperm production have been demonstrated following chronic boron exposure. There are clinical and biochemical tests to detect neurological and gonadal injury, but these are not specific for boron exposure. Sparse data in animals suggest some biochemical changes; for instance, cerebral succinate dehydrogenase was increased in rats after boron exposure. Animal data further demonstrate biochemical alterations following gonadal injury. Dose-dependent reduction in hyaluronidase, sorbitol dehydrogenase, and lactic acid dehydrogenase (isoenzyme-X) were observed in rats following boron exposure.

\subsection{INTERACTIONS WITH OTHER CHEMICALS}

No studies were located regarding the influence of other chemicals on the toxicity of boron.

\subsection{POPULATIONS THAT ARE UNUSUALLY SUSCEPTIBLE}

A susceptible population will exhibit a different or enhanced response to boron than will most persons exposed to the same level of boron in the environment. Reasons may include genetic makeup, age, health and nutritional status, and exposure to other toxic substances (e.g., cigarette smoke). These parameters result in reduced detoxification or excretion of boron, or compromised function of organs affected by boron. Populations who are at greater risk due to their unusually high exposure to boron are discussed in Section 6.7, Populations with Potentially High Exposures.

No data were located identifying a population that is unusually susceptible to boron toxicity. Case reports in humans suggest that large variability exists with the human population to the lethal effect of boron. However, there are no data to suggest which segment of the population is more susceptible to boron. 


\subsection{METHODS FOR REDUCING TOXIC EFFECTS}

This section will describe clinical practice and research concerning methods for reducing toxic effects of exposure to boron. However, because some of the treatments discussed may be experimental and unproven, this section should not be used as a guide for treatment of exposures to boron. When specific exposures have occurred, poison control centers and medical toxicologists should be consulted for medical advice. The following texts provide specific information about treatment following exposures to boron:

Ellenhorn MJ, Schonwald S, Ordog G, et al., eds. 1997. Ellenhorn's medical toxicology. Diagnosis and treatment of human poisoning. 2nd ed. Baltimore, MA: Williams \& Wilkins, 1098-1100, 160t, $162 \mathrm{t}$.

Goldfrank LR, Flomenbaum NE, Lewin NA, et al., eds. 2002. Goldfrank's toxicologic emergencies. 7th ed. New York, NY: McGraw-Hill, 1282, 1289-1290, 1134.

Viccellio P, Bania T, Brent J, et al., eds. 1998. Emergency toxicology. 2nd ed. Philadelphia, PA: Lippincott-Raven, 448-449, 470, 1141.

Human exposure to boron may occur by inhalation, ingestion, or dermal contact (see Chapter 6). Boron in the form of boric acid or borate dust is an upper respiratory tract irritant following inhalation and may also irritate the eyes and skin. Ingestion of boron may cause gastrointestinal, neurological, hepatic, renal, and dermal effects (see Section 3.2). General recommendations for reducing absorption of boron following exposure have included removing the exposed individual from the contaminated area and removing the contaminated clothing. If the eyes and skin were exposed, then they should be flushed with water.

\subsubsection{Reducing Peak Absorption Following Exposure}

Nausea, vomiting, and diarrhea have been induced by ingestion of boron in humans. Some authors recommend reducing absorption of boron from the gastrointestinal tract by administration of emetics (e.g., syrup of ipecac) and cathartics (e.g., magnesium sulfate) (Stewart and McHugh 1990). Caution should be taken, however, not to induce further damage to the esophageal mucosa or to cause aspiration of the vomit into the lungs during emesis. There is disagreement regarding the efficiency of activated charcoal in preventing absorption of boron from the gastrointestinal tract following oral exposure (Ellenhorn and Barceloux 1988; Stewart and McHugh 1990). It has been suggested that activated charcoal be administered following gastric evacuation, but its effectiveness has not been established 
(Ellenhorn and Barceloux 1988). Administration of intravenous fluids may be required if severe dehydration or shock develop and local skin care may be necessary if skin desquamation occurs (Stewart and McHugh 1990). In addition, the treatment of boron poisoning may require a control for convulsions.

\subsubsection{Reducing Body Burden}

Elemental boron is not metabolized (see Section 3.4). Studies in volunteers indicated that most of the administered dose is excreted in the urine within few days (Jansen et al. 1984a). Saline diuresis has been suggested to enhance urinary excretion of boron (Goldfrank et al. 1990). Exchange transfusions, peritoneal dialysis, or hemodialysis may be employed to lower plasma boron levels following either acute or chronic intoxication. There are indications that hemodialysis is the most effective of these procedures (Goldfrank et al. 1990; Naderi and Palmer 2006; Stewart and McHugh 1990).

\subsubsection{Interfering with the Mechanism of Action for Toxic Effects}

No studies were available to support measures to interfere with the mechanism of action for boron once it has been absorbed.

\subsection{ADEQUACY OF THE DATABASE}

Section 104(I)(5) of CERCLA, as amended, directs the Administrator of ATSDR (in consultation with the Administrator of EPA and agencies and programs of the Public Health Service) to assess whether adequate information on the health effects of boron is available. Where adequate information is not available, ATSDR, in conjunction with the National Toxicology Program (NTP), is required to assure the initiation of a program of research designed to determine the health effects (and techniques for developing methods to determine such health effects) of boron.

The following categories of possible data needs have been identified by a joint team of scientists from ATSDR, NTP, and EPA. They are defined as substance-specific informational needs that if met would reduce the uncertainties of human health assessment. This definition should not be interpreted to mean that all data needs discussed in this section must be filled. In the future, the identified data needs will be evaluated and prioritized, and a substance-specific research agenda will be proposed. 


\subsubsection{Existing Information on Health Effects of Boron}

The existing data on health effects of inhalation, oral, and dermal exposure of humans and animals to boron are summarized in Figure 3-4. The purpose of this figure is to illustrate the existing information concerning the health effects of boron. Each dot in the figure indicates that one or more studies provide information associated with that particular effect. The dot does not necessarily imply anything about the quality of the study or studies, nor should missing information in this figure be interpreted as a "data need”. A data need, as defined in ATSDR's Decision Guide for Identifying Substance-Specific Data Needs Related to Toxicological Profiles (Agency for Toxic Substances and Disease Registry 1989), is substance-specific information necessary to conduct comprehensive public health assessments. Generally, ATSDR defines a data gap more broadly as any substance-specific information missing from the scientific literature.

Most of the information concerning health effects of oral exposure to boron in humans is found in case reports of accidental acute and intermediate ingestion of boron. Controlled-exposure studies of volunteers and cross-sectional surveys of borate mining and production workers have identified acute upper respiratory and ocular irritation as an effect of concern from acute inhalation exposure. Epidemiology studies of intermediate- to chronic-duration exposures (involving repeated occupational exposure to dusts of borates or repeated exposure to boron in drinking water) have not clearly identified a toxic effect in humans, but have found no associations between boron exposure and impaired pulmonary function or impaired fertility. Information on acute dermal exposure exists, but none was found on effects after intermediate- and chronic-duration exposure.

In animals, information exists on health effects following acute, intermediate, and chronic oral exposure to boric acid or borax. Comprehensive acute toxicity studies involving inhalation exposure to boron compounds are not available, with the exception of a study of mice exposed by inhalation to diborane gas for 2 or 4 weeks. Diborane gas is expected to have a very short half-life in the environment because of its reactivity. Thus, it is not expected to be a significant environmental toxicant, except in workplaces where it might be used and accidentally released. Health effects have been examined in rats and dogs exposed by inhalation to boric oxide aerosols for intermediate durations. Studies of health effects in animals exposed by inhalation to boron compounds for chronic durations are not available. Health effects have been observed in animals following acute dermal exposure, but no toxicity studies of animals dermally exposed for intermediate and chronic durations are available. 
Figure 3-4. Existing Information on Health Effects of Boron
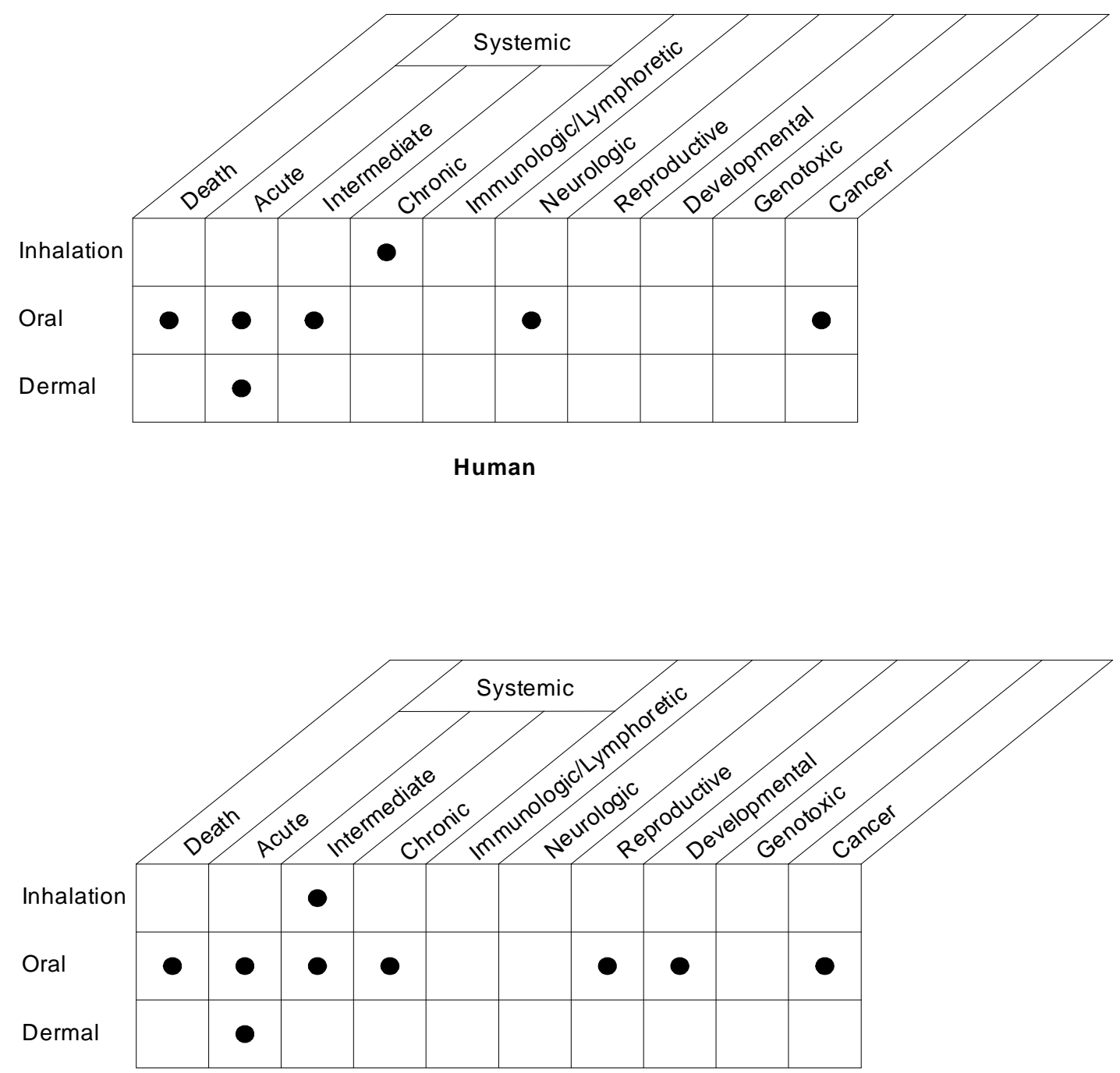

Animal

Existing Studies 


\subsubsection{Identification of Data Needs}

Acute-Duration Exposure. Associations between acute inhalation exposure to borate dusts and increased prevalence of self-reported symptoms of irritation of the eyes, nose, and throat of volunteers under controlled exposure conditions (Cain et al. 2004, 2008) form the basis of an acute-duration inhalation MRL. These data are supported by self-reported data for mild upper respiratory tract irritation in workers under workplace conditions (Garabrant et al. 1984, 1985; Hu et al. 1992; Wegman et al. 1991, 1994). Dose-response relationships for mild respiratory and ocular irritation from studies of animals exposed by inhalation for acute durations are not available.

Case reports of acute oral poisonings with boric acid do not clearly identify toxicity targets and doseresponse relationships in humans, but reported effects in fatal cases include degenerative changes in the liver, kidney, and brain (Chao et al. 1991a, 1991b; Wong et al. 1964). In animal studies, acute-duration oral exposure to boric acid or borates has been associated with effects on the male reproductive organs (e.g., decreased testicular weight and altered sperm morphology) in Wistar rats exposed to gavage boric acid doses of $88 \mathrm{mg}$ boron/kg/day for 2 weeks (Fukuda et al. 2000; Kudo et al. 2000) and developmentally toxic effects (including reduced fetal weight and increased skeletal variations or malformations) in CD-1 mice exposed during gestation to boric acid doses as low as $70 \mathrm{mg}$ boron $/ \mathrm{kg}$ (2 times/day) (Cherrington and Chernoff 2002) and in New Zealand rabbits exposed during gestation (days 6-19) to doses of $44 \mathrm{mg}$ boron/kg/day (Price et al. 1996b). A NOAEL of $22 \mathrm{mg}$ boron/kg/day for developmental effects in rabbits (Price et al. 1996b) serves as the basis of the acute-duration oral MRL for boron.

Dermal/ocular effects have been associated with dermal exposure in humans (Beckett et al. 2001) and animals (Wilding et al. 1959). The irritation effects observed in animals may have been due to the exothermic rehydration reaction of the anhydride boron oxide.

No further acute-duration health effect studies are recommended at this time.

Intermediate-Duration Exposure. No studies are available that establish associations between intermediate-duration oral exposures to boron compounds and health effects in humans. Case reports of intermediate-duration exposure to humans are limited to reports on infants exhibiting neurological effects after ingestion of high levels of boron as boric acid. Seizure disorders occurred during oral exposures of 
infants to borax lasting 4-12 weeks, with cumulative approximate doses of 12-120 g (Gordon et al. 1973; O'Sullivan and Taylor 1983). No associations were found between elevated levels of borates in drinking water and fertility rates in surveys of Turkish subpopulations expected to have intermediate- to chronicduration exposures to boron (Sayli 1998a, 1998b, 2003; Sayli et al. 1998). Likewise, cross-sectional surveys of boron mining and processing workers in California (Whorton et al. 1994) and China (Chang et al. 2006; Liu et al. 2006a, 2006b) failed to find associations between boron exposure (which may have included oral exposure to boron) and adverse effects on indices of fertility. Robbins et al. (2008) associated a shift in Y:X sperm and male:female offspring ratios with boron exposure; however, this study had multiple confounders limiting the interpretation of reported results.

Studies in animals indicate that reproductive and developmental effects are the most sensitive effects associated with intermediate-duration oral exposures to boric acid or borates. Testicular atrophy and histopathology, sperm abnormalities, and reduced sperm production have been observed in mice, rats and dogs after intermediate-duration ingestion of doses $\geq 26 \mathrm{mg}$ boron/kg/day as boric acid (Dieter 1994; Dixon et al. 1976, 1979; Fail et al. 1991; Fukuda et al. 2000; Harris et al. 1992; Ku et al. 1993a; Kudo et al. 2000; Lee et al. 1978; NTP 1987; Nusier and Bataineh 2005; Seal and Weeth 1980; Weir and Fisher 1972; Yoshizaki et al. 1999). Complete sterility was observed in Sprague-Dawley rats fed boric acid or borax in the diet (101 and $116 \mathrm{mg}$ boron/kg/day for males and females, respectively) for 14 weeks before mating; sterility was associated with a lack of viable sperm in atrophied testes in males and decreased ovulation in females (Weir and Fisher 1972). No pregnancies occurred, when female rats exposed to this dose level were mated with non-exposed male rats. At lower exposure levels (10 or $30 \mathrm{mg}$ boron/kg/day for males and 12 or $35 \mathrm{mg}$ boron/kg/day for females), no exposure-related adverse effects were found on overall fertility indices in three successive generations (Weir and Fisher 1972). Developmental effects (including decreased fetal weight, increased incidence of skeletal variations and malformations, and increased resorptions) have been observed in offspring of rat and mouse dams exposed to 13-79 mg boron/kg/day as boric acid during gestation (Heindel et al. 1992, 1994; Price et al. 1996a). Multiple developmental end point data from the rat studies by Heindel et al. (1992) and Price et al. (1996a) were pooled and subjected to benchmark dose analyses (Allen et al. 1996) to derive a benchmark-dose point of departure of $10.3 \mathrm{mg}$ boron/kg/day for the intermediate-duration oral MRL for boron.

Studies of Chinese borate workers have evaluated specific clinical end points of semen quality, but do not provide adequate estimation of magnitude or duration of boron exposure. Additional cross-sectional or prospective surveys of reproductive health end points in populations exposed to elevated levels of boron compounds in drinking water may help to better identify reproductive toxicity and developmental toxicity 
as potential health effects in humans with elevated oral exposure to boron for intermediate or chronic durations.

Increased frequencies of symptoms of acute upper respiratory and ocular irritation have been reported in workers exposed repeatedly by inhalation to boron oxide and borate dusts at average concentrations of 1.8 and $3.1 \mathrm{mg}$ boron $/ \mathrm{m}^{3}$, and employed in the borax industry for a mean duration of 11.4 years (SD 8.1 years), compared with a reference population (Garabrant et al. 1984, 1985); however, it is uncertain if these acute symptoms were due to acute or repeated exposure. Later studies of a different design indicated that acute irritation symptoms in these workers are due to acute exposures, and that pulmonary function variables (e.g., $\mathrm{FEV}_{1}$ ) were not significantly influenced by exposure over a 7-year period of employment (Hu et al. 1992; Wegman et al. 1994). These results are adequate to form the basis of an acute inhalation MRL for boron, but do not clearly indicate whether the dose-response relationships for acute boron-induced upper respiratory and ocular irritation symptoms are changed with intermediate or chronic durations of exposure. Because exposure-related effects on pulmonary function variables were not evident during a 7-year period between the studies of Garabrant et al. $(1984,1985)$ and Wegman et al. (1994), the results provide some confidence that the acute-duration inhalation MRL may by protective for intermediate- and chronic-duration exposures.

Intermediate-duration inhalation exposure studies in animals are restricted to a series of studies in which albino rats were exposed to boric oxide aerosols for 6 hours/day, 5 days/week for 10 weeks at an average concentration of $73 \mathrm{mg}$ boron $/ \mathrm{m}^{3}$ ( $\mathrm{n}=20$ rats), 12 weeks at $27 \mathrm{mg}$ boron $/ \mathrm{m}^{3}$ ( $\left.\mathrm{n}=4\right)$, or 24 weeks at $12 \mathrm{mg}$ boron $/ \mathrm{m}^{3}$ ( $\mathrm{n}=70$ ) (Wilding et al. 1959). Histopathologic examination of a comprehensive set of tissues (lung, trachea, pancreas, thyroid, adrenal, eye, femur, rib, bone marrow, liver, heart, spleen, kidney, brain, stomach, intestine, ovary, testis, lymph node, and muscle) in exposed and control rats revealed no exposure-related lesions, with the exception that some rats exposed to the highest concentration (73 mg boron $/ \mathrm{m}^{3}$ ) showed a reddish nasal exudate. Although dogs were included in this study (three dogs were exposed to $9 \mathrm{mg}$ boron $/ \mathrm{m}^{3}$ for 23 weeks), end points were restricted to clinical signs of toxicity, body weight, hematological end points, and sulfobromophthalein retention, a measure of liver function (Wilding et al. 1959). No exposure-related effects on these end points were found in exposed dogs, compared with controls. Because the NOAELs identified in the rat and dog studies were higher than concentrations associated with acute respiratory and ocular irritation in humans acutely exposed to boron (Cain et al. 2004; Wegman et al. 1994), the intermediate-duration inhalation database was considered inadequate for derivation of an MRL. 
Additional prospective health evaluations of respiratory function and reproductive and developmental variables in borate mining and processing workers with intermediate- and chronic-duration exposures may help to better identify impaired respiratory function, impaired reproductive performance, and developmental effects as critical effects in humans from intermediate- or chronic duration exposure to boron compounds.

Chronic-Duration Exposure and Cancer. No studies are available that establish associations between chronic-duration oral exposures to boron compounds and noncancer or cancer effects in humans. As discussed in the previous section, no associations were found between exposure to borates or boric oxide and indices of fertility in surveys of Turkish subpopulations expected to have intermediate- to chronic-duration oral exposures to boron (Sayli 1998a, 1998b, 2003; Sayli et al. 1998), or in surveys of boron mining and processing workers in California (Whorton et al. 1994) and China (Chang et al. 2006; Liu et al. 2006a, 2006b). Chronic-duration oral toxicity studies in animals include 2-year toxicity studies in Sprague-Dawley rats and beagle dogs exposed to boric acid or borax in the diet (Weir and Fisher 1972), and a 2-year toxicity and cancer bioassay in B6C3F1 mice (Dieter 1994; NTP 1987).

Histopathological examination of a comprehensive set of tissues from exposed and control rats and dogs (brain, pituitary, thyroids, lung, heart, liver spleen, kidneys, adrenals, pancreas, intestines, urinary bladder testes, ovary (rat only), bone, and bone marrow) found no exposure-related non-neoplastic or neoplastic lesions at dose levels up to $6.8 \mathrm{mg}$ boron/ $\mathrm{kg} /$ day in dogs or $81 \mathrm{mg}$ boron/kg/day in rats, with the exception that rats exposed to $81 \mathrm{mg}$ boron/kg/day, but not $24 \mathrm{mg}$ boron/kg/day, showed testicular atrophy, decreased growth, decreased packed blood cell and hemoglobin levels, and scaly tails and desquamated footpad skin (Weir and Fisher 1972). In the mouse bioassay, no cancer responses were observed at dose levels up to $201 \mathrm{mg}$ boron/kg/day (Dieter 1994; NTP 1987). Noncancer effects in mice included testicular atrophy and interstitial hyperplasia, lung hemorrhage, and 10-17\% decreased body weight at $201 \mathrm{mg}$ boron/kg/day and splenic hematopoiesis and chronic hepatic inflammation and coagulative necrosis at 79 and $201 \mathrm{mg}$ boron/kg/day, the only dose levels included in the study.

Although data are sufficient to develop a chronic oral MRL, a value was not derived. Because intermediate-duration LOAELs for developmental toxicity in rats (13.6 and $13 \mathrm{mg}$ boron/kg/day [Heindel et al. 1992; Price et al. 1996a, 1998]) were lower than the NOAEL (24 mg boron/kg/day) for testicular atrophy and other non-cancer effects in chronically exposed rats (Weir and Fisher 1972), the intermediate MRL, which is based on developmental toxicity, should be protective against reproductive toxicity following chronic exposure. In mice, the intermediate-duration oral NOAEL (43.4 mg boron/kg/day) and LOAEL (79 mg boron/kg/day) for developmental toxicity in CD-1 mice (Heindel et al. 1992) were lower 
than the chronic-duration LOAEL for testicular atrophy and decreased body weight in B6C3F1 mice (201 mg boron/kg/day) and overlapped with the LOAEL (79 mg boron/kg/day) for splenic hematopoiesis and chronic hepatic inflammation and necrosis (Dieter 1994; NTP 1987). Additional studies may be useful in assessing the level of confidence in existing intermediate-duration NOAEL and LOAEL values for developmental toxicity in rats and mice. Additional chronic-duration oral exposure studies in animals do not seem necessary at this time.

As discussed in the previous section, symptoms of acute upper respiratory and ocular irritation have been reported in workers employed in the borax industry for an average of $>10$ years, but pulmonary function variables were not significantly influenced by exposure over a 7-year period of employment (Garabrant et al. 1984, 1985; Hu et al. 1992; Wegman et al. 1994). These results are the basis of an acute inhalation MRL for boron, but do not clearly indicate whether the dose-response relationships for acute boroninduced upper respiratory and ocular irritation effects are changed with intermediate or chronic durations of exposure. Because exposure-related effects on pulmonary function variables were not evident during a 7-year period of employment, the results provide some confidence that the acute-duration inhalation MRL may by protective for intermediate- and chronic-duration exposures. Additional prospective health evaluations of respiratory function and reproductive and developmental variables in borate mining and processing workers with intermediate- and chronic-duration exposures may help to better identify impaired respiratory function, impaired reproductive performance, and developmental effects as critical effects in humans from intermediate- or chronic-duration inhalation exposure to boron compounds.

The available chronic oral bioassays in rats and dogs exposed to boric acid or borax (Weir and Fisher 1972) and mice exposed to boric acid (Dieter 1994; NTP 1987) found no evidence for exposure-related cancer. Although no epidemiological studies have been conducted to examine possible associations between boron exposure and cancer, the results from the animal studies provide no strong impetus to conduct such studies.

Genotoxicity. No in vivo human data were located. Bacterial and limited mammalian assays were negative for mutagenicity (Benson et al. 1984; Landolph 1985; Demerec et al. 1951; Haworth et al. 1983; NTP 1987) or cell transformation (Landolph 1985). However, the in vivo animal study of Wery et al. (2003) reported specific alterations in gene expression in embryos at critical points of development that may be associated with skeletal malformations seen in several animal species. Thus, additional in vivo studies may be useful to establish whether direct genetic changes or indirect factors are responsible for the observed changes in gene expression associated with observed skeletal effects in animals. 
Reproductive Toxicity. No associations were found between exposure to borates or boric oxide and indices of fertility in surveys of Turkish subpopulations expected to have intermediate- to chronicduration oral exposures to boron (Sayli 1998a, 1998b, 2003; Sayli et al. 1998), or in surveys of boron mining and processing workers in California (Whorton et al. 1994) and China (Chang et al. 2006). Studies of Chinese borate workers and populations living in environments with high boron exposure did not find effects on semen quality (Liu et al. 2006a, 2006b); however, adequate estimation magnitude or duration of boron exposure for each study group was not available. One study of Chinese borate workers associated high boron exposure with a shift toward males in the ratio the sex of offspring (Robbins et al. 2008); however, several confounding factors in this study limit the interpretation of study data.

Effects on the male and female reproductive organs have been clearly demonstrated in rats orally exposed to boric acid or borax; supporting evidence for effects on the male reproductive organs from oral exposure to boron has been reported in mice and dogs. Testicular atrophy and histopathology, sperm abnormalities, and reduced sperm production have been observed in mice, rats, and dogs after intermediate-duration ingestion of doses $\geq 26 \mathrm{mg}$ boron/kg/day as boric acid (Dieter 1994; Dixon et al. 1976, 1979; Fail et al. 1991; Fukuda et al. 2000; Harris et al. 1992; Ku et al. 1993a; Kudo et al. 2000; Lee et al. 1978; NTP 1987; Nusier and Bataineh 2005; Seal and Weeth 1980; Weir and Fisher 1972; Yoshizaki et al. 1999). Complete sterility was observed in male and female Sprague-Dawley rats fed boric acid or borax in the diet for 14 weeks before mating at 101 and $116 \mathrm{mg}$ boron/kg/day for males and females, respectively (Weir and Fisher 1972). Sterility was associated with a lack of viable sperm in males and decreased ovulation in females, and no pregnancies occurred when female rats exposed to this dose level were mated with non-exposed male rats. At lower exposure levels (10 or $30 \mathrm{mg}$ boron/kg/day for males and 12 or $35 \mathrm{mg}$ boron/kg/day for females), no exposure-related adverse effects were found on fertility indices in three successive generations (Weir and Fisher 1972). With chronic-duration oral exposure, testicular atrophy occurred at 201, but not 79, mg boron/kg/day in B6C3F1 mice (Dieter 1994; NTP 1987) and at 81, but not 24, mg boron/kg/day in Sprague-Dawley rats (Weir and Fisher 1972). No testicular atrophy was found in dogs exposed for 2 years to dietary doses of boric acid or borax at levels up to $6.8 \mathrm{mg}$ boron/kg/day (Weir and Fisher 1972). In the chronic-duration oral exposure studies, no histologic effects on the ovaries were found in rats (Weir and Fisher 1972) or mice (Dieter 1994; NTP 1987), but the ovaries of chronically exposed female dogs were not examined (Weir and Fisher 1972).

With inhalation exposure to boron compounds, reproductive effects do not appear to be an effect of concern. In intermediate-duration inhalation studies with rats, no histologic effects on the testes or ovaries were found in albino rats exposed to boric oxide aerosols for 6 hours/day, 5 days/week for 
10 weeks at an average concentration of $73 \mathrm{mg}$ boron $/ \mathrm{m}^{3}, 12$ weeks at $27 \mathrm{mg}$ boron $/ \mathrm{m}^{3}$, or 24 weeks at $12 \mathrm{mg}$ boron $/ \mathrm{m}^{3}$ (Wilding et al. 1959).

Additional prospective health evaluations of reproductive variables in borate mining and processing workers with intermediate- and chronic-duration exposures may help to better identify impaired reproductive performance as a critical effects in humans from intermediate- or chronic-duration inhalation exposure to boron compounds.

Developmental Toxicity. No studies were found on the developmental effects of boron and compounds in humans following inhalation, oral, or dermal exposure. In acute-duration oral exposure animal studies, developmentally toxic effects (including reduced fetal weight and increased skeletal variations or malformations) have been reported in CD-1 mice exposed during gestation to boric acid doses as low as $70 \mathrm{mg}$ boron/kg (2 times/day) (Cherrington and Chernoff 2002) and in New Zealand rabbits exposed during gestation (days 6-19) to doses of $44 \mathrm{mg}$ boron/kg/day (Price et al. 1996b). Developmental effects (including decreased fetal weight, increased incidence of skeletal variations and malformations, and increased resorptions) have been observed in offspring of rat and mouse dams exposed to 13-79 mg boron/kg/day as boric acid during gestation for intermediate durations (Heindel et al. 1992, 1994; Price et al. 1996a). Developmental effects observed in these animal studies are the critical effects for the acute- and intermediate-duration oral MRLs for boron.

Developmental toxicity studies in animals exposed to boron compounds by inhalation are not available. Such studies may be useful to determine if developmental effects are a critical effect from inhalation exposure to boron compounds.

Immunotoxicity. No studies were found in humans or animals on the effects of boron on the immune system by any route of exposure. Results of chronic studies do not suggest that the immune system is a potential target for boron toxicity. Additional studies are not needed at this time.

Neurotoxicity. Case reports in humans, primarily infants, indicate that neurological effects occur after ingestion of boron at high dose levels (Wong et al. 1964). Degenerative changes in brain cells, perivascular hemorrhage, and intravascular thrombosis have been reported in fatal case reports in infants, but neurochemical or neurophysiological changes have not been reported (Settimi et al. 1982; Wong et al. 1964). No studies are available on neurotoxic effects of boron following inhalation or dermal exposure in humans. Animal data are limited to increased brain enzyme activity (Settimi et al. 1982), but no 
histopathological data are available. Since data on effects are limited primarily to acute oral exposures at high dose levels, additional studies in animals evaluating other dose levels and exposure routes and durations may be useful in evaluating potential risk to humans who may be exposed to low levels of boron compounds near hazardous waste sites.

Epidemiological and Human Dosimetry Studies. Information exists on the adverse health effects of boron compounds in humans. Symptoms of acute upper respiratory and ocular irritation have been reported in workers employed in the borax industry for an average of $>10$ years, but pulmonary function variables were not significantly influenced by exposure over a 7-year period of employment (Garabrant et al. 1984, 1985; Hu et al. 1992; Wegman et al. 1991, 1994). More precise quantitation of the irritation potential of airborne boron compounds comes from controlled exposure studies of human volunteers (Cain et al. 2004, 2008). No associations were found between exposure to borates or boric oxide and indices of fertility in surveys of Turkish subpopulations expected to have intermediate- to chronic-duration oral exposures to boron (Sayli 1998a, 1998b, 2003; Sayli et al. 1998), or in surveys of boron mining and processing workers in California (Whorton et al. 1994) and China (Chang et al. 2006). Studies of Chinese borate workers and populations living in environments with high boron exposure did not find effects on semen quality (Liu et al. 2006a, 2006b). Other human studies involve case reports of accidental or intentional ingestion of large quantities of boron compounds (Wong et al. 1964; Litovitz et al. 1988; Locatelli et al. 1987). The case report studies identified key health effects (gastrointestinal, respiratory, renal, neurological, hepatic) associated with boron exposure (Wong et al. 1964).

Results from animal studies indicate that the testes and developing fetus as the most sensitive targets following acute, intermediate, or chronic oral exposure to boron. Epidemiological studies that look for associations between boron exposure and reproductive and/or developmental toxicity end points would be useful to better identify impaired reproductive performance and developmental effects as critical effects in humans from intermediate- or chronic-duration exposure to boron compounds.

\section{Biomarkers of Exposure and Effect.}

Exposure. Blood and urine borate concentrations may be useful biomarkers of exposure (Jansen et al. 1984a; Litovitz et al. 1988). Normal dietary concentrations of boron in the blood of humans range from 0 to $1.25 \mu \mathrm{g} / \mathrm{mL}$ in children and infants (Fisher and Freimuth 1958; O'Sullivan and Taylor 1983).

Elevated blood levels of boron have been reported in cases of acute ingestion of boric acid (Linden et al. 1986; Wong et al. 1964). Urinary concentrations of boron in the normal population range from 0.07 to 
$0.15 \mathrm{mg} / 100 \mathrm{~mL}$ (Vignec and Ellis 1954) and from 0.004 to $0.66 \mathrm{mg} / 100 \mathrm{~mL}$ (Imbus et al. 1963), whereas elevated concentrations have been measured in humans orally exposed to borax or boric acid (Gordon et al. 1973; Jansen et al. 1984a). Additional studies of exposure levels and blood or urinary levels of boron in borate mining and production workers may help to better characterize quantitative relationships between occupational exposure levels and blood or urinary levels of boron.

Effect. The most clearly identified effects in humans exposed to boron compounds are acute respiratory and ocular irritation from acute inhalation exposure to boron compounds. Several other types of effects, including degenerative changes in brain cells, gastrointestinal irritation, degenerative liver or kidney lesions, and skin changes (erythema involving palms, soles, and buttocks), have been observed in some, but not all, cases of acute- or intermediate-duration oral poisoning with boric acid or borax. In orally exposed animals, effects include testicular atrophy in males and decreased ovulation in females, and developmental effects (fetal body weight deficits and skeletal malformations) with gestational exposure. None of these effects, however, are necessarily specific to boron. To date, a specific biomarker of effect for boron has not been developed for humans or animals.

Absorption, Distribution, Metabolism, and Excretion. Limited quantitative information is available on the absorption, distribution, metabolism, and elimination of boron compounds following oral (Dourson et al. 1998; Ku et al. 1991; Moseman 1994; Treinen and Chapin 1991), inhalation (Wilding et al. 1959), and dermal (Draize and Kelley 1959; Wester et al. 1998) exposure. Since data on toxicokinetics of boron are limited, additional studies are needed by all routes of exposure that will provide data on absorption rates, amount and rate of accumulation in various tissues, and clearance rates. Limited data from oral and dermal studies suggest that boron is primarily excreted in urine. Since boron can deposit in the upper respiratory tract, additional excretion studies by this route would be useful in determining if excretion patterns are similar across all routes of exposure.

Comparative Toxicokinetics. Existing evidence from human and animal studies do not indicate whether or not boron compounds affect the same target tissues. Animal studies indicate the testes as a target tissue (Dieter 1994; Dixon et al. 1979; Fukuda et al. 2000; Ku et al. 1993a; Kudo et al. 2000; Lee et al. 1978; NTP 1990; Price et al. 1998; Seal and Weeth 1980; Weir and Fisher 1972). However, studies of Chinese borate workers found no effect of boron exposure on multiple indices of semen quality (Lui et al. 2006a, 2006b). Data exist on excretion of boron compounds. Based on excretion studies, boron compounds are absorbed by the gastrointestinal tract. There are no available quantitative toxicokinetics 
data on absorption, distribution, and metabolism. Additional toxicokinetics studies may provide a better basis for extrapolation of animal data to human exposure risk.

Methods for Reducing Toxic Effects. Methods for the mitigation of acute effects of boron poisoning include prevention of absorption of boron from the gastrointestinal tract and standard procedures used to prevent convulsions, severe dehydration, or shock (Stewart and McHugh 1990). Saline diuresis, exchange transfusions, peritoneal dialysis, or hemodialysis may be employed to enhance removal of absorbed boron from the body (Goldfrank et al. 1990; Stewart and McHugh 1990). No additional information was located concerning mitigation of effects of lower-level or longer-term exposure to boron. Further information on techniques to mitigate such effects may be useful in determining the safety and effectiveness of possible methods for treating boron-exposed populations in the vicinity of hazardous waste sites.

Children's Susceptibility. Data needs relating to both prenatal and childhood exposures, and developmental effects expressed either prenatally or during childhood, are discussed in detail in the Developmental Toxicity subsection above.

The comparative susceptibility of children to the acute respiratory and ocular irritation potential of aerosols of boric acid or other boron compounds has not been examined. Results from animal studies indicate that developmental effects associated with gestational exposure are the most sensitive effects associated with acute- or intermediate-duration oral exposures. Degenerative changes in the male (e.g., testicular atrophy and altered spermiation) and female (decreased ovulation) have also been identified as a sensitive effect from boron, but no studies were located that examined the relative susceptibility of young animals (or children) to these effects.

Child health data needs relating to exposure are discussed in Section 6.8.1, Identification of Data Needs: Exposures of Children.

\subsubsection{Ongoing Studies}

Wendie A. Robbins of the University of California Los Angeles is being funded by the NIOSH to perform an epidemiologic study investigating the relationship between workplace exposure to boroncontaining compounds (including boric acid, borax) and adverse male reproductive effects. Two published human studies on reproductive effects of occupational boron exposure found no effect on 
fertility or development, while one study reported positive testicular atrophy and sterility and another associated a shift in the ratio of offspring sex with boron exposure. All of these studies have been criticized for inadequacies in study design exposure assessment. The specific aims of this research are to: (1) describe the relationship between boron exposure and direct measures of toxicity on male reproduction, (2) describe the relationship between boron exposure and indirect measures of toxicity on male reproduction, and (3) describe the relationship between workplace, environmental, and dietary sources of boron with biomarkers of exposure and reproductive effect. The goal is to contribute critical information on the exposure level at which boron causes adverse effects on human male reproduction. 
3. HEALTH EFFECTS

This page is intentionally blank. 


\section{CHEMICAL AND PHYSICAL INFORMATION}

\subsection{CHEMICAL IDENTITY}

Boron appears in Group 13 (IIIA) of the periodic table and is the only nonmetal of this group (Jansen 2003). Table 4-1 lists common synonyms, trade names, and other pertinent information to identify boron and selected compounds.

\subsection{PHYSICAL AND CHEMICAL PROPERTIES}

Boron is a nonmetal and is typically found in nature bound to oxygen. It is never found as the free element (Cotton et al. 1999). Boron has two stable isotopes, ${ }^{10} \mathrm{~B}$ and ${ }^{11} \mathrm{~B}$, which are naturally present at 19.10-20.31 and 79.69-80.90\%, respectively (Jansen 2003). There are ten other known non-radioactive isotopes of boron (Lide 2008). Elemental boron exists in several allotropic forms (Jansen 2003). In addition to being an amphorous powder, boron has four crystalline forms: $\alpha$-rhombohedral, $\beta$-rhombohedral, $\alpha$-tetragonal, and $\beta$-tetragonal (Jansen 2003).

The chemical properties of boron are more similar to carbon and silicon than elements of its own group, although boron is more electron deficient. Boron has a high affinity for oxygen-forming borates, and reacts with water at temperatures above $100{ }^{\circ} \mathrm{C}$ to form boric acid and other boron compounds. The electron deficiency of boron does not allow conventional two-electron bonds, but rather multicenter bonds that are exclusively covalent (Jansen 2003; Schubert and Brotherton 2006). Boron hydrides consequently have structures quite different from hydrocarbons (Jansen 2003). The multi-center nature of boron allows the formation of a diverse array of covalent networks, rings, cages, and clusters. Boron forms strong covalent bonds with electronegative elements such as fluorine and oxygen (Schubert and Brotherton 2006).

Table 4-2 lists important physical and chemical properties of boron and selected compounds. 


\section{Table 4-1. Chemical Identity of Boron and Selected Boron Compounds ${ }^{a}$}

\begin{tabular}{llll}
\hline Characteristic & Boron & Boron oxide & Boric acid \\
\hline Synonym(s) & Boron, metallic & $\begin{array}{l}\text { Boric anhydride; boric } \\
\text { acid, anhydride; boron } \\
\text { sesquioxide; boron } \\
\text { trioxide; diboron trioxide; } \\
\text { fused boric acid }\end{array}$ & $\begin{array}{l}\text { Orthoboric acid; boron } \\
\text { hydroxide; boron }\end{array}$ \\
trihydroxide
\end{tabular}




\section{Table 4-1. Chemical Identity of Boron and Selected Boron Compounds ${ }^{a}$}

\begin{tabular}{|c|c|c|c|}
\hline Characteristic & Borax & Sodium tetraborate & Boron tribromide \\
\hline Synonym(s) & $\begin{array}{l}\text { Sodium borate; sodium } \\
\text { tetraborate; borax } \\
\text { decahydrate; disodium } \\
\text { tetraborate decahydrate }\end{array}$ & $\begin{array}{l}\text { Sodium borate; sodium } \\
\text { borate anhydrous; } \\
\text { disodium tetraborate; } \\
\text { sodium biborate; sodium } \\
\text { pyroborate; boric acid, } \\
\text { disodium salt }\end{array}$ & $\begin{array}{l}\text { Boron bromide; tribromo- } \\
\text { borane; tribromoboron }\end{array}$ \\
\hline $\begin{array}{l}\text { Registered trade } \\
\text { name(s) }\end{array}$ & $\begin{array}{l}\text { Jaikin, Pyrobor, Three } \\
\text { Elephant, V-Bor }{ }^{b}\end{array}$ & Dehybor $65^{b}$ & No data \\
\hline Chemical formula & $\mathrm{B}_{4} \mathrm{Na}_{2} \mathrm{O}_{7} \cdot 10 \mathrm{H}_{2} \mathrm{O}$ & $\mathrm{Na}_{2} \mathrm{~B}_{4} \mathrm{O}_{7}$ & $\mathrm{BBr}_{3}$ \\
\hline Chemical structure & $\begin{array}{l}\text { Tetraborate anion in } \\
\text { solution }^{\mathrm{d}}\end{array}$ & $\begin{array}{l}\text { Anhydrous borates have } \\
\text { polymeric assemblies of } \\
\text { planar } \mathrm{BO}_{3} \text { and/or } \\
\text { tetrahedral } \mathrm{BO}_{4} \text { units } \\
\text { linked by shared oxygen } \\
\text { atoms }{ }^{d}\end{array}$ & $3 r$ \\
\hline \multicolumn{4}{|l|}{ Identification numbers: } \\
\hline CAS registry & $1303-96-4$ & $1330-43-4$ & $10294-33-4$ \\
\hline NIOSH RTECS ${ }^{\mathrm{e}}$ & VZ2275000 & ED4588000 & ED7400000 \\
\hline EPA hazardous waste & No data & No data & No data \\
\hline $\begin{array}{l}\text { EPA/OPP Pesticide } \\
\text { Code }\end{array}$ & 029601 & 011112 & No data \\
\hline $\mathrm{OHM} / \mathrm{TADS}$ & No data & No data & No data \\
\hline $\begin{array}{l}\text { DOT/UN/NA/IMDG } \\
\text { shipping }\end{array}$ & No data & No data & UN 2692; IMDG 8.1 \\
\hline HSDB & 328 & 5025 & 327 \\
\hline EINECS & $233-139-2$ & $215-540-4$ & $233-657-9$ \\
\hline $\mathrm{NCl}$ & No data & No data & No data \\
\hline
\end{tabular}




\section{Table 4-1. Chemical Identity of Boron and Selected Boron Compounds ${ }^{a}$}

\begin{tabular}{|c|c|c|}
\hline Characteristic & Boron trifluoride & Boron trichloride \\
\hline Synonym(s) & $\begin{array}{l}\text { Anca 1040; boron fluoride; } \\
\text { trifluoroborane }\end{array}$ & $\begin{array}{l}\text { Trichloroborane; } \\
\text { trichloroboron }\end{array}$ \\
\hline $\begin{array}{l}\text { Registered trade } \\
\text { name(s) }\end{array}$ & No data & No data \\
\hline Chemical formula & $\mathrm{BF}_{3}$ & $\mathrm{BCl}_{3}$ \\
\hline Chemical structure & $F$ & $\mathrm{Cl}$ \\
\hline \multicolumn{3}{|l|}{ Identification numbers: } \\
\hline CAS registry & $7637-07-2$ & $10294-34-5$ \\
\hline NIOSH RTECS ${ }^{e}$ & ED2275000 & ED1925000 \\
\hline EPA hazardous waste & No data & No data \\
\hline $\begin{array}{l}\text { EPA/OPP Pesticide } \\
\text { Code }\end{array}$ & No data & No data \\
\hline OHM/TADS & No data & No data \\
\hline $\begin{array}{l}\text { DOT/UN/NA/IMDG } \\
\text { shipping }\end{array}$ & $\begin{array}{l}\text { UN 1008; IMDG } 6.1 \\
\text { (boron trifluoride); UN } \\
\text { 2851; IMDG } 8.2 \text { (boron } \\
\text { trifluoride dihydrate) }\end{array}$ & UN 1741; IMDG 2.2 \\
\hline HSDB & 325 & 326 \\
\hline EINECS & $231-569-5$ & $233-658-4$ \\
\hline $\mathrm{NCl}$ & No data & No data \\
\hline
\end{tabular}

${ }^{a}$ All information obtained from HSDB 2009 and ChemIDplus 2009, except where noted.

${ }^{\mathrm{b}} \mathrm{NIOSH} 2005$

c Jansen 2003

${ }^{\mathrm{d}}$ Cotton et al. 1999

${ }^{\mathrm{e}} \mathrm{RTECS} 2007$

CAS = Chemical Abstracts Service; DOT/UN/NA/IMDG = Department of Transportation/United Nations/North America/Intergovernmental Maritime Dangerous Goods Code; EINECS = European Inventory of Existing Chemical Substances; EPA = Environmental Protection Agency; HSDB = Hazardous Substances Data Bank; $\mathrm{NCl}=\mathrm{National}$ Cancer Institute; NIOSH = National Institute for Occupational Safety and Health; OHM/TADS = Oil and Hazardous Materials/Technical Assistance Data System; RTECS = Registry of Toxic Effects of Chemical Substances 


\section{Table 4-2. Physical and Chemical Properties of Boron and Selected Boron Compounds $^{\mathrm{a}}$}

\begin{tabular}{|c|c|c|c|}
\hline Property & Boron & Boron oxide & Boric acid \\
\hline Molecular weight & 10.811 & 69.620 & 61.833 \\
\hline $\begin{array}{l}\text { Physical } \\
\text { description }\end{array}$ & $\begin{array}{l}\text { Black or dark brown powder } \\
\text { (amorphous form); clear red } \\
\text { crystals ( } \alpha \text {-rhombohedral } \\
\text { form); black, opaque } \\
\text { crystals with metallic luster } \\
\text { ( } \alpha \text {-tetragonal form); black } \\
\text { ( } \beta \text {-rhombohedral form) }\end{array}$ & $\begin{array}{l}\text { Colorless, glassy or } \\
\text { hexagonal crystals, } \\
\text { hygroscopic }\end{array}$ & $\begin{array}{l}\text { Colorless, transparent } \\
\text { crystals; white granules or } \\
\text { powder }\end{array}$ \\
\hline Melting point & $2,075^{\circ} \mathrm{C}$ & $450^{\circ} \mathrm{C}$ (crystal) & $170.9^{\circ} \mathrm{C}$ \\
\hline Boiling point & $4,000{ }^{\circ} \mathrm{C}$ & $1,500^{\circ} \mathrm{C}$ (crystal) & No data \\
\hline Density & $\begin{array}{l}2.350 \mathrm{~g} / \mathrm{cm}^{3} \text { (amorphous); } \\
2.46 \mathrm{~g} / \mathrm{cm}^{3} \\
\text { ( } \alpha \text {-rhombohedral); } \\
2.31 \mathrm{~g} / \mathrm{cm}^{3} \text { ( } \alpha \text {-tetragonal); } \\
2.35 \mathrm{~g} / \mathrm{cm}^{3} \\
\text { ( } \beta \text {-rhombohedral) }\end{array}$ & $\begin{array}{l}1.8 \mathrm{~g} / \mathrm{cm}^{3} \text { (amorphous); } \\
2.46 \mathrm{~g} / \mathrm{cm}^{3} \text { (crystal) }\end{array}$ & $1.435 \mathrm{~g} / \mathrm{cm}^{3}$ at $15^{\circ} \mathrm{C}$ \\
\hline Odor & No data & Odorless & Odorless \\
\hline \multicolumn{4}{|l|}{ Solubility: } \\
\hline Water & Insoluble & $4.0 \%$ at $20^{\circ} \mathrm{C}$ & $50 \mathrm{~g} / \mathrm{L}$ at $25^{\circ} \mathrm{C}$ \\
\hline $\begin{array}{l}\text { Organic } \\
\text { solvent(s) }\end{array}$ & Insoluble alcohol, ether & $\begin{array}{l}\text { Soluble in alcohol, } \\
\text { glycerol }\end{array}$ & $\begin{array}{l}17.5 \% \text { in glycerol; } 18.5 \% \text { in } \\
\text { ethylene glycol; } 173.9 \mathrm{~g} / \mathrm{L} \text { in } \\
\text { methanol; } 94.4 \mathrm{~g} / \mathrm{L} \text { in ethanol; } \\
0.6 \% \text { in acetone; } 1.5 \% \text { in ethyl } \\
\text { acetate (all at } 25{ }^{\circ} \mathrm{C} \text { ) }\end{array}$ \\
\hline Other & $\begin{array}{l}\text { Soluble in concentrated } \\
\text { nitric and sulfuric acids }\end{array}$ & No data & No data \\
\hline $\mathrm{pK}_{\mathrm{a}}$ & No data & No data & 9.42 \\
\hline $\log K_{o w}$ & No data & No data & 0.175 \\
\hline Vapor pressure & $0.0119 \mathrm{~mm} \mathrm{Hg}$ at $2,140{ }^{\circ} \mathrm{C}$ & Negligible at $20^{\circ} \mathrm{C}$ & Negligible at $20^{\circ} \mathrm{C}$ \\
\hline $\begin{array}{l}\text { Autoignition } \\
\text { temperature }\end{array}$ & $580^{\circ} \mathrm{C}$ & No data & No data \\
\hline Flashpoint & No data & No data & No data \\
\hline $\begin{array}{l}\text { Flammability } \\
\text { limits in air }\end{array}$ & $\begin{array}{l}\text { Dust ignites spontaneously } \\
\text { in air }\end{array}$ & Noncombustible & Not flammable \\
\hline $\begin{array}{l}\text { Conversion } \\
\text { factors }\end{array}$ & No data & No data & No data \\
\hline Explosive limits & No data & No data & No data \\
\hline
\end{tabular}




\section{Table 4-2. Physical and Chemical Properties of Boron and Selected Boron} Compounds $^{\mathrm{a}}$

\begin{tabular}{|c|c|c|c|}
\hline Property & Borax & Sodium tetraborate & Boron tribromide \\
\hline Molecular weight & 381.373 & 201.220 & 250.52 \\
\hline $\begin{array}{l}\text { Physical } \\
\text { Description }\end{array}$ & White, monoclinic crystals & $\begin{array}{l}\text { Colorless glassy solid; } \\
\text { hygroscopic }\end{array}$ & Colorless, fuming liquid \\
\hline Melting point & $75^{\circ} \mathrm{C}$ (decomposes) & $743^{\circ} \mathrm{C}$ & $-46.0^{\circ} \mathrm{C}$ \\
\hline Boiling point & No data & $1,575^{\circ} \mathrm{C}$ (decomposes) & $90^{\circ} \mathrm{C}$ \\
\hline Density & $1.73 \mathrm{~g} / \mathrm{cm}^{3}$ & $2.367 \mathrm{~g} / \mathrm{cm}^{3}$ & $2.698 \mathrm{~g} / \mathrm{cm}^{3}$ at $0^{\circ} \mathrm{C}$ \\
\hline Odor & Odorless & Odorless & Sharp, irritating odor, pungent \\
\hline \multicolumn{4}{|l|}{ Solubility: } \\
\hline Water & $59.3 \mathrm{~g} / \mathrm{L}$ at $25^{\circ} \mathrm{C}$ & $3.1 \%$ at $25^{\circ} \mathrm{C}$ & Decomposes in water \\
\hline $\begin{array}{l}\text { Organic } \\
\text { solvents }\end{array}$ & $\begin{array}{l}1 \mathrm{~g} / 1 \mathrm{~mL} \text { in glycerol; } \\
\text { insoluble in alcohol }\end{array}$ & $\begin{array}{l}16.7 \% \text { in methanol; } 30 \% \\
\text { in ethylene glycol; } \\
40.6 \mathrm{~g} / \mathrm{L} \text { in formamide (all } \\
\text { at } 25^{\circ} \mathrm{C} \text { ) }\end{array}$ & $\begin{array}{l}\text { Decomposes in alcohol; } \\
\text { soluble in carbon } \\
\text { tetrachloride, sulfur dioxide } \\
\text { (liquid), carbon disulfide }\end{array}$ \\
\hline Other & Insoluble in acid & No data & No data \\
\hline $\mathrm{pK}_{\mathrm{a}}$ & No data & No data & No data \\
\hline $\log K_{o w}$ & No data & No data & No data \\
\hline Vapor pressure & Negligible & Negligible at $20^{\circ} \mathrm{C}$ & $100 \mathrm{~mm} \mathrm{Hg}$ at $33.5^{\circ} \mathrm{C}$ \\
\hline $\begin{array}{l}\text { Autoignition } \\
\text { temperature }\end{array}$ & No data & No data & No data \\
\hline Flashpoint & No data & No data & No data \\
\hline $\begin{array}{l}\text { Flammability } \\
\text { limits in air }\end{array}$ & Not flammable & Noncombustible & Nonflammable \\
\hline $\begin{array}{l}\text { Conversion } \\
\text { factors }\end{array}$ & No data & No data & $1 \mathrm{ppm}=10.25 \mathrm{mg} / \mathrm{m}^{3 \mathrm{~b}}$ \\
\hline Explosive limits & No data & No data & No data \\
\hline
\end{tabular}




\section{Table 4-2. Physical and Chemical Properties of Boron and Selected Boron Compounds $^{\mathrm{a}}$}

\begin{tabular}{|c|c|c|}
\hline Property & Boron trifluoride & Boron trichloride \\
\hline Molecular weight & 67.81 & 117.17 \\
\hline $\begin{array}{l}\text { Physical } \\
\text { Description }\end{array}$ & Colorless gas & Colorless fuming liquid at low temperature \\
\hline Melting point & $-126.8^{\circ} \mathrm{C}$ & $-107^{\circ} \mathrm{C}$ \\
\hline Boiling point & $-101^{\circ} \mathrm{C}$ & $12.5^{\circ} \mathrm{C}$ \\
\hline Density & $3.07666 \mathrm{~g} / \mathrm{L}$ at $1 \mathrm{~atm}, 0^{\circ} \mathrm{C}$ & $1.3728 \mathrm{~g} / \mathrm{cm}^{3}$ at $0{ }^{\circ} \mathrm{C}$ \\
\hline Odor & Pungent, suffocating odor, irritating ${ }^{c}$ & Pungent, suffocating odor, irritating, sharp \\
\hline \multicolumn{3}{|l|}{ Solubility: } \\
\hline Water & $\begin{array}{l}332 \mathrm{~g} / 100 \mathrm{~g} \text { water at } 0{ }^{\circ} \mathrm{C} \text { with some } \\
\text { hydrolysis forming fluoboric and boric } \\
\text { acids }\end{array}$ & Decomposes in water, hydrolyzed easily \\
\hline $\begin{array}{l}\text { Organic } \\
\text { solvents }\end{array}$ & $\begin{array}{l}\text { Soluble in benzene, dichlorobenzene, } \\
\text { chloroform, carbon tetrachloride, carbon } \\
\text { sulfide; soluble in most saturated and } \\
\text { halogenated hydrocarbons and in } \\
\text { aromatic compounds }\end{array}$ & Decomposes in alcohol \\
\hline Other & $\begin{array}{l}1.94 \mathrm{~g} / 100 \mathrm{~g} \text { in anhydrous sulfuric acid, } \\
\text { soluble in concentrated nitric acid }\end{array}$ & No data \\
\hline $\mathrm{pK}_{\mathrm{a}}$ & No data & No data \\
\hline $\log K_{\text {ow }}$ & No data & No data \\
\hline Vapor pressure & $3.656 \times 10^{4} \mathrm{~mm} \mathrm{Hg}$ at $-13.2^{\circ} \mathrm{C}$ & $1 \mathrm{~mm} \mathrm{Hg}$ at $25^{\circ} \mathrm{C}$ (extrapolated) \\
\hline $\begin{array}{l}\text { Autoignition } \\
\text { temperature }\end{array}$ & No data & No data \\
\hline Flashpoint & No data & No data \\
\hline $\begin{array}{l}\text { Flammability } \\
\text { limits in air }\end{array}$ & Nonflammable & Nonflammable \\
\hline $\begin{array}{l}\text { Conversion } \\
\text { factors }\end{array}$ & $1 \mathrm{ppm}=2.77 \mathrm{mg} / \mathrm{m}^{3 \mathrm{~b}}$ & No data \\
\hline Explosive limits & No data & No data \\
\hline
\end{tabular}

${ }^{a}$ All information from HSDB 2009, except where noted.

${ }^{\mathrm{b}} \mathrm{NIOSH} 2009$

${ }^{\mathrm{c} O d o r}$ threshold $4.50 \mathrm{mg} / \mathrm{m}^{3}$ (Ruth 1986) 
4. CHEMICAL AND PHYSICAL INFORMATION

This page is intentionally blank. 


\section{PRODUCTION, IMPORT/EXPORT, USE, AND DISPOSAL}

\subsection{PRODUCTION}

Boron is the $51^{\text {st }}$ most common element found in the earth's crust and is found at an average concentration of $8 \mathrm{mg} / \mathrm{kg}$ (Cotton et al. 1999; Jansen 2003). Boron is a nonmetal and is typically found in nature bound to oxygen. It is never found as the free element (Cotton et al. 1999). There are over 200 minerals containing boron oxide; however, only four boron-containing minerals (borax, kernite, colemite, and ulexite) comprise the majority, nearly $90 \%$, of the borates used by industry worldwide. These minerals are extracted mainly from California and Turkey. The majority of domestic boron production is from Kern County, California, with the remainder from San Bernardino and Inyo Counties in California (USGS 2008).

The most widely used commercial process for producing boron is the Moissan process, which involves the reduction of boric oxide with magnesium (Jansen 2003). This process yields 90-92\% pure boron, which is then leached with acid to separate it from the magnesium oxide formed during the process, followed by multiple washes and drying. The purity of the boron can be increased to $95-97 \%$ by further chemical processing (Jansen 2003). Due to boron's tendency to bind to electron-rich elements (carbon, nitrogen, and oxygen) it can be very difficult to isolate boron in high purity (Cotton et al. 1999). High purity boron ( $>99.9 \%$ ) is prepared by the reduction of boron trihalides or by the decomposition of boron triiodide or boron hydrides at high temperatures. Other methods include electrolytic reduction of potassium tetrafluoroborate $\left(\mathrm{KBF}_{4}\right)$ in molten potassium chloride-potassium fluoride mixtures. High purity boron can generally only be obtained in kilogram quantities (Cotton et al. 1999).

In 2005, 1.15 million metric tons of boron ore were produced in the United States, with a boron oxide $\left(\mathrm{B}_{2} \mathrm{O}_{3}\right)$ content of 612,000 metric tons. Colemanite, kernite, tincal (natural borax), and ulexite were the most common mineral of commercial importance in the United States. Boron compounds and minerals are produced both by surface and underground mining, as well as from brine (USGS 2008).

Boron trifluoride is prepared by the reaction of a boron-containing material and a fluorine-containing substance in the presence of an acid (e.g., borax, fluorspar, and sulfuric acid) (Alam et al. 2003). It can also be produced by the treatment of fluorosulfonic acid with boric acid. Large-scale production of boron trichloride involves the reaction of chlorine with a mixture of borax and crude oil residue heated in a rotary kiln. On a smaller-scale, boron trichloride can be prepared by reacting chlorine and a mixture of boron oxide, petroleum coke, and lampblack (carbon black) in a fluidized bed. Large-scale production of 
boron tribromide involves reaction of bromine and granulated boron carbide $\left(\mathrm{B}_{4} \mathrm{C}\right)$ at $850-1,000{ }^{\circ} \mathrm{C}$ or by reaction of hydrogen bromide with calcium boride $\left(\mathrm{CaB}_{6}\right)$ at high temperatures (Alam et al. 2003).

Tables 5-1 and 5-2 list facilities in each state that manufacture or process boron trifluoride and boron trichloride, respectively, as well as the intended use and the range of maximum amounts of these boron compounds that are stored onsite. In 2006, there were 50 and 19 reporting facilities that produced, processed, or used boron trifluoride and boron trichloride, respectively, in the United States. The data listed in Tables 5-1 and 5-2 are derived from the Toxics Release Inventory (TRI06 2008). Only certain types of facilities were required to report. Therefore, this is not an exhaustive list. Current U.S. manufacturers of boron and selected boron compounds are given in Table 5-3.

\subsection{IMPORT/EXPORT}

Turkey was a major import source in 2004-2007 for boric acid, supplying 55\%, followed by Chile (24\%), Bolivia (8\%), Peru (5\%), and other (8\%) (USGS 2009). In 2008, U.S. imports of borax, boric acid, colemanite, and ulexite were $1 \times 10^{3}, 65 \times 10^{3}, 27 \times 10^{3}$, and $90 \times 10^{3}$ metric tons, respectively. In 2008, U.S. exports of boric acid and refined sodium borates were $260 \times 10^{3}$ and $470 \times 10^{3}$ metric tons, respectively (USGS 2009).

\subsection{USE}

In 2008, the estimated use distribution pattern for boron compounds in the United States was $74 \%$ for glass and ceramics, $6 \%$ for soaps and detergents and bleaches, $3 \%$ for agriculture, $3 \%$ for enamels and glazes, and 14\% remaining for other (USGS 2009). Boric acid is used in cosmetics, pharmaceuticals, and toiletries. It is also used to reduce the flammability of cellulose insulation, cotton batting in mattresses, and wood composites. Boron oxide is incorporated into cellulose materials to inhibit combustion. Borates are used in the manufacture of adhesives and are added to lubricants, brake fluids, metalworking fluids, water treatment chemicals, and fuel additives (USGS 2008).

Pesticide products containing boric acid and its sodium salts (sodium tetraborate decahydrate, sodium tetraborate pentahydrate, anhydrous sodium tetraborate, disodium octaborate tetrahydrate, anhydrous disodium octaborate, and sodium metaborate) are registered in the United States for use as insecticides, fungicides, and herbicides. There are 189 pesticide products registered that contain boric acid or its 
Table 5-1. Facilities that Produce, Process, or Use Boron Trifluoride

\begin{tabular}{|c|c|c|c|c|}
\hline State $^{a}$ & $\begin{array}{l}\text { Number of } \\
\text { facilities }\end{array}$ & $\begin{array}{l}\text { Minimum } \\
\text { amount on site } \\
\text { in pounds }\end{array}$ & $\begin{array}{l}\text { Maximum } \\
\text { amount on site } \\
\text { in pounds }^{b}\end{array}$ & Activities and uses ${ }^{c}$ \\
\hline$\overline{A L}$ & 4 & 1,000 & 99,999 & $2,3,6,10$ \\
\hline AR & 3 & 1,000 & 99,999 & $2,3,6$ \\
\hline DE & 2 & 100,000 & 999,999 & $1,4,8$ \\
\hline $\mathrm{FL}$ & 3 & 0 & 99,999 & 6,10 \\
\hline KY & 2 & 10,000 & 99,999 & 10,12 \\
\hline LA & 5 & 0 & 999,999 & $2,3,10,11$ \\
\hline MD & 2 & 1,000 & 99,999 & 6 \\
\hline NJ & 2 & 0 & 9,999 & 6 \\
\hline NY & 3 & 1,000 & 999,999 & 6 \\
\hline $\mathrm{OH}$ & 1 & 10,000 & 99,999 & 6 \\
\hline OK & 3 & 10,000 & 999,999 & $1,3,6$ \\
\hline PA & 8 & 0 & 999,999 & $6,7,9,10,12$ \\
\hline SC & 6 & 1,000 & 99,999 & $2,3,6,12$ \\
\hline $\mathrm{TN}$ & 1 & 10,000 & 99,999 & 10 \\
\hline TX & 13 & 0 & 999,999 & $2,3,4,6,9,10,11,12$ \\
\hline
\end{tabular}

${ }^{a}$ Post office state abbreviations used

${ }^{\mathrm{b}}$ Amounts on site reported by facilities in each state ${ }^{\mathrm{c}}$ Activities/Uses:
1. Produce
6. Impurity
2. Import
7. Reactant
11. Chemical Processing Aid
3. Onsite use/processing
8. Formulation Component
12. Manufacturing Aid
4. Sale/Distribution
9. Article Component
13. Ancillary/Other Uses
5. Byproduct
10. Repackaging
14. Process Impurity

Source: TRI07 2009 (Data are from 2007) 


\section{Table 5-2. Facilities that Produce, Process, or Use Boron Trichloride}

\begin{tabular}{|c|c|c|c|c|}
\hline State $^{a}$ & $\begin{array}{l}\text { Number of } \\
\text { facilities }\end{array}$ & $\begin{array}{l}\text { Minimum } \\
\text { amount on site } \\
\text { in pounds }\end{array}$ & $\begin{array}{l}\text { Maximum } \\
\text { amount on site } \\
\text { in pounds }\end{array}$ & Activities and uses ${ }^{c}$ \\
\hline$\overline{A Z}$ & 1 & 100 & 999 & 11,12 \\
\hline CA & 2 & 1,000 & 99,999 & $1,2,3,4,9$ \\
\hline IN & 3 & 10,000 & 999,999 & $6,7,10,11$ \\
\hline MA & 2 & 1,000 & 9,999 & $2,3,6$ \\
\hline MI & 1 & 1,000 & 9,999 & 10 \\
\hline NM & 2 & 100 & 9,999 & 11 \\
\hline NV & 1 & 0 & 0 & 0 \\
\hline $\mathrm{OH}$ & 1 & 1,000 & 9,999 & 6 \\
\hline PA & 4 & 0 & 99,999 & $2,4,9$ \\
\hline SC & 2 & 1,000 & 9,999 & $2,3,6,7,10,11,12$ \\
\hline WI & 2 & 1,000 & 99,999 & 6 \\
\hline WV & 1 & 10,000 & 99,999 & 6 \\
\hline
\end{tabular}

${ }^{\mathrm{a}}$ Post office state abbreviations used

${ }^{\mathrm{b}}$ Amounts on site reported by facilities in each state ${ }^{\mathrm{c}}$ Activities/Uses:
1. Produce
6. Impurity
2. Import
7. Reactant
11. Chemical Processing Aid
3. Onsite use/processing
8. Formulation Component
12. Manufacturing Aid
4. Sale/Distribution
9. Article Component
13. Ancillary/Other Uses
5. Byproduct
10. Repackaging
14. Process Impurity

Source: TRI07 2009 (Data are from 2007) 
Table 5-3. Current U.S. Manufacturers of Boron and Selected Boron Compounds

\begin{tabular}{ll}
\hline Company & Location \\
\hline Boron & \\
Eagle-Picher Incoroporated, Eagle-Pitcher Boron LLC & Quapaw, Oklahoma \\
SB Boron Corporation & $\begin{array}{l}\text { Franklin Park, Illinois } \\
\text { TETRA Micronutrients }\end{array}$ \\
$\begin{array}{l}\text { Fairbury, Nebraska } \\
\text { Hronox Incorporated }\end{array}$ & \\
Boron oxide & Ward Hill, Massachusetts \\
Johnson Matthey, Inc., Alfa Aesar & \\
Boric acid & Phoenix, Arizona \\
InCide ${ }^{8}$ Technologies, Inc. & Trona, California \\
Searles Valley Minerals, Argus-Trona-Westend Complex & Boron, California \\
Rio Tinto Minerals & \\
Sodium tetraborate pentahydrate (borax pentahydrate) & Westend, California \\
Searles Valley Minerals, Argus-Trona-Westend Complex & Boron, California \\
Rio Tinto Minerals & \\
Sodium tetraborate decahydrate (borax decahydrate) & Westend, California \\
Searles Valley Minerals, Argus-Trona-Westend Complex & Boron, California \\
Rio Tinto Minerals & \\
Sodium tetraborate (Borax) & Trona, California \\
Searles Valley Minerals, Argus-Trona-Westend Complex & Westend, California \\
Rio Tinto Minerals & Boron, California \\
Boron tribromide & \\
Air Liquide America L.P., Air Liquide Electronics Division & Dallas, Texas \\
EP Scientific Products, LLC & Miami, Oklahoma \\
Schumacher & Carlsbad, California \\
Boron trifluoride dihydrate & \\
Atotech USA, Inc. & Rock Hill, South Carolina \\
Boron trichloride & \\
Tronox Incorporated & Henderson, Nevada \\
\hline
\end{tabular}

Source: Stanford Research Institute (SRI 2008), except where otherwise noted. SRI reports production of chemicals produced in commercial quantities (defined as exceeding 5,000 pounds or $\$ 10,000$ in value annually) by the companies listed. 
sodium salt as an active ingredient. Boric acid and its sodium salts are used on several agricultural and many non-agricultural sites including residential, commercial, medical, veterinary, industrial, forestry, and food/feed handling areas. Various formulations are available, including liquids, soluble and emulsifiable concentrates, granulars, powders, dusts, pellets, tablets, solids, paste, baits, and crystalline rods (EPA 1993).

Boron halides are important industrial chemicals. Their Lewis acid properties make them useful as catalysts. Boron trichloride is widely used to prepare boron filaments by chemical vapor deposition (CVD). Much of the boron tribromide produced in the United States is used in the manufacture of proprietary pharmaceuticals (Alam et al. 2003).

Boron isotopes have also found several medical and analytical uses. BNCT, a biologically targeted form of radiotherapy, offers the potential use of the non-radioactive, stable ${ }^{10} \mathrm{~B}$ isotope for cancer imaging. This technique may allow selective delivery of ${ }^{10} \mathrm{~B}$ to tumors and, as a result, better targeted irradiation of the tumor (Schubert and Brotherton 2006; Wittig et al. 2008). The measured ratio of ${ }^{10} \mathrm{~B}$ to ${ }^{11} \mathrm{~B}$ is frequently used as a hydrologic and geochemical tracer (Chetelat and Gaillardet 2005; Moore et al. 2008). The natural abundance of ${ }^{10} \mathrm{~B}$ and ${ }^{11} \mathrm{~B}$ is specific to location, making anthropogenic boron isotopic ratios from a mined location distinctly different than that found in most groundwater and soil ratios (Davidson and Bassett 1993). This allows utilization of the boron isotopic ratio to determine potential contamination in groundwater (Vengosh et al. 1994), river water (Chetelat and Gaillardet 2005), marine water (Xiao et al. 2007), and effluent (Kloppmann et al. 2008).

\subsection{DISPOSAL}

Boron trifluoride and boron trichloride are classified as extremely hazardous substances under the Comprehensive Environmental Response, Compensation, and Liability Act of 1980 (CERCLA), commonly known as Superfund, and the Superfund Amendments and Reauthorization Act (SARA) of 1986 and the Emergency Planning and Community Right-to-Know Act (EPCRA), also known as Title III of SARA. Under CERCLA, spills or discharges into the environment of more than 500 pounds of boron trifluoride or boron trichloride must be reported immediately to the National Response Center (EPA 2008b).

Boron trifluoride and boron trichloride are also regulated under the chemical accident prevention provisions of the Clean Air Act (CAA) amendments of 1990. Owners and operators of stationary sources 
5. PRODUCTION, IMPORT/EXPORT, USE, AND DISPOSAL

who produce, process, handle, or store boron trifluoride in excess of 5,000 pounds are required to initiate specific activities to prevent and mitigate accidental releases (i.e., hazard assessment, a prevention program, and an emergency response program) (EPA 2008a).

Boron recycling in the United States during 2008 was insignificant (USGS 2009). No other information regarding disposal of boron or other boron compounds was located. 
5. PRODUCTION, IMPORT/EXPORT, USE, AND DISPOSAL

This page is intentionally blank. 


\section{POTENTIAL FOR HUMAN EXPOSURE}

\subsection{OVERVIEW}

Boron and boron compounds have been identified in at least 164 of the 1,689 hazardous waste sites that have been proposed for inclusion on the EPA National Priorities List (NPL) (HazDat 2007). However, the number of sites evaluated for boron and boron compounds is not known. The frequency of these sites can be seen in Figure 6-1. Of these sites containing boron and boron compounds, 163 are located within the United States and 1 is located in Guam (not shown).

Boron is the $51^{\text {st }}$ most common element found in the earth's crust and is found in an average concentration of $8 \mathrm{mg} / \mathrm{kg}$ (approximately 0.0008\%) (Cotton et al. 1999; Jansen 2003). Boron is a nonmetal and is typically found in nature bound to oxygen. It is never found as the free element (Cotton et al. 1999). There are over 200 minerals containing boron oxide; the four most important boroncontaining minerals are borax, kernite, colemite, and ulexite (USGS 2008). Boron is an essential micronutrient for most plants and there is evidence that it is also essential for animals, including humans (Rainey et al. 1999, 2002).

In 2008, the primary use of boron compounds in the United States was for glass and ceramics, followed by soaps and detergents, bleaches, agriculture, and enamels and glazes (USGS 2009). Boric acid is used in cosmetics, pharmaceuticals, and toiletries. It is also used to reduce the flammability of cellulose insulation, cotton batting in mattresses, and wood composites. Boron oxide is also incorporated into cellulose materials to inhibit combustion. Borates are used in the manufacture of adhesives and are added to lubricants, brake fluids, metalworking fluids, water treatment chemicals, and fuel additives (USGS 2008). There are 189 pesticide products registered in the United States that contain boric acid or its sodium salt as an active ingredient (EPA 1993).

Borates are widespread, naturally-occurring substance found mainly as inorganic compounds in sediments and sedimentary rock. Boron is released to the environment slowly in low concentrations by weathering processes. Although few data are available quantifying boron releases from industrial sources, it is estimated that natural weathering releases more boron to the environment worldwide than do these industrial sources (Butterwick et al. 1989). 
Figure 6-1. Frequency of NPL Sites with Boron and Selected Boron Contamination

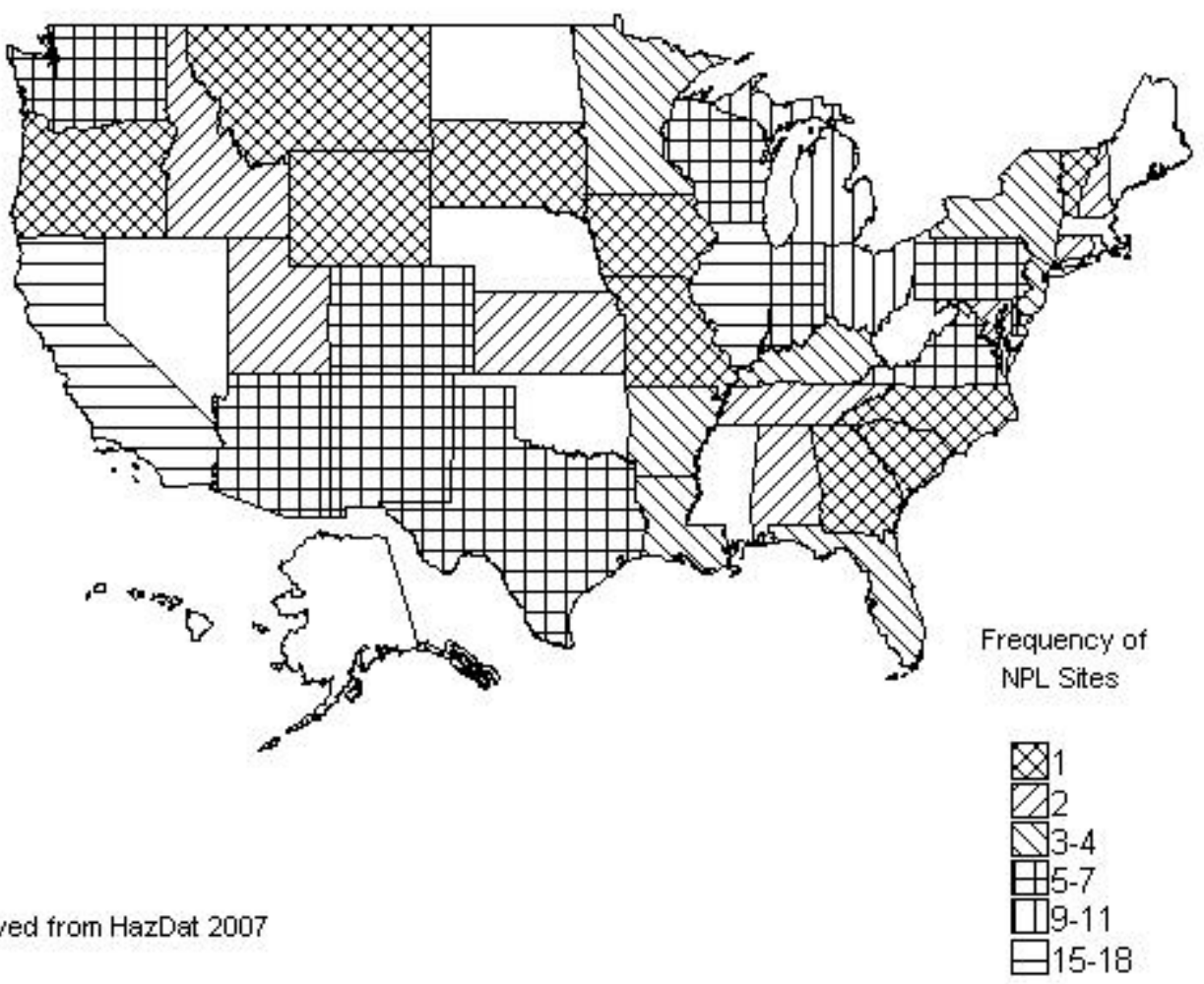


Boron can be released from municipal sewage waste water, coal-burning power plants, copper smelters, and industries using boron compounds. Boron can also be released from runoff where boron-containing fertilizers and herbicides are used (Butterwick et al. 1989; Fox et al. 2002; Nolte 1988; Waggott 1969).

Adsorption-desorption reactions are expected to be the only significant mechanism that will influence the fate of boron in water (Rai et al. 1986). The extent of boron adsorption depends on the $\mathrm{pH}$ of the water and the chemical composition of the soil. The greatest adsorption is generally observed at $\mathrm{pH}$ 7.59.0 (Keren and Mezuman 1981; Keren et al. 1981; Waggott 1969). The abundance of amorphous aluminum oxide in soil is the single-most important property of soil that will influence the mobility of boron (Bingham et al. 1971).

Rainey et al. (2002) reported mean daily intakes of boron for male and female adults to be 1.28 and $1.00 \mathrm{mg}$, respectively, from food and beverages. Daily dietary boron intakes were $0.75 \mathrm{mg}$ for infants aged 0-6 months and $0.99 \mathrm{mg}$ for infants aged 7-11 months. Daily boron intakes were $0.86 \mathrm{mg}$ for children aged 1-3 years, $0.80 \mathrm{mg}$ for children aged 4-8 years, 0.90 and $1.02 \mathrm{mg}$, respectively, for adolescent males aged 9-13 and 14-18 years, and 0.83 and $0.78 \mathrm{mg}$, respectively, for adolescent females aged 9-13 and 14-18 years.

Ingestion of boron from food (primarily fruits and vegetables) and water is the most frequent route of human exposure, but occupational exposures to boron dusts may be significant. Boron is also a component of several consumer products, including cosmetics medicines and insecticides. Populations residing in areas of the western United States with natural boron-rich deposits may be exposed to higherthan-average levels of boron.

Boron is widely distributed in surface water and groundwater. An average surface water boron concentration in the United States is about $0.1 \mathrm{mg} / \mathrm{L}$ (Butterwick et al. 1989; EPA 1986b), but concentrations vary greatly, depending on boron content of local geologic formations and anthropogenic sources of boron (Butterwick et al. 1989). A survey of U.S. surface waters detected boron in $98 \%$ of 1,577 samples at concentrations ranging from 0.001 to $5 \mathrm{mg} / \mathrm{L}$ (Butterwick et al. 1989). Concentrations of boron in tap water have been reported in a range of $0.007-0.2 \mathrm{mg} / \mathrm{L}$ in the United States, Canada, and England (Choi and Chen 1979; Davies 1990; Waggott 1969). In a 1987 survey of 989 public water supplies, boron concentrations ranged from $<0.005$ to $>2 \mathrm{mg} / \mathrm{L}$ (NIRS 1987). Mean boron concentrations in soil in the United States are about $30 \mathrm{mg} / \mathrm{kg}$, with concentrations ranging up to $300 \mathrm{mg} / \mathrm{kg}$ (Eckel and Langley 1988; USGS 1984). 


\subsection{RELEASES TO THE ENVIRONMENT}

The Toxics Release Inventory (TRI) data should be used with caution because only certain types of facilities are required to report (EPA 2005). This is not an exhaustive list. Manufacturing and processing facilities are required to report information to the TRI only if they employ 10 or more full-time employees; if their facility is included in Standard Industrial Classification (SIC) Codes 10 (except 1011, 1081, and 1094), 12 (except 1241), 20-39, 4911 (limited to facilities that combust coal and/or oil for the purpose of generating electricity for distribution in commerce), 4931 (limited to facilities that combust coal and/or oil for the purpose of generating electricity for distribution in commerce), 4939 (limited to facilities that combust coal and/or oil for the purpose of generating electricity for distribution in commerce), 4953 (limited to facilities regulated under RCRA Subtitle C, 42 U.S.C. section 6921 et seq.), 5169, 5171, and 7389 (limited S.C. section 6921 et seq.), 5169, 5171, and 7389 (limited to facilities primarily engaged in solvents recovery services on a contract or fee basis); and if their facility produces, imports, or processes $\geq 25,000$ pounds of any TRI chemical or otherwise uses $>10,000$ pounds of a TRI chemical in a calendar year (EPA 2005).

\subsubsection{Air}

Estimated releases of 3,600 pounds ( 1.6 metric tons) of boron trifluoride to the atmosphere from 21 domestic manufacturing and processing facilities in 2006, accounted for about $100 \%$ of the estimated total environmental releases from facilities required to report to the TRI (TRI06 2008). Estimated releases of 520 pounds ( $\sim 24$ metric tons) of boron trichloride to the atmosphere from five domestic manufacturing and processing facilities in 2006, accounted for about $100 \%$ of the estimated total environmental releases from facilities required to report to the TRI (TRI06 2008). These releases for boron trifluoride and boron trichloride are summarized in Tables 6-1 and 6-2, respectively. There is no information on releases of other boron compounds to the atmosphere from manufacturing and processing facilities because these releases are not required to be reported (EPA 1997).

Borates are released to air from natural and industrial sources. Natural sources include oceans, volcanoes, and geothermal steam (Graedel 1978). Boron compounds are released from anthropogenic sources such as coal-fired and geothermal steam power plants, chemical plants, and rockets as well as manufacturing 


\section{Table 6-1. Releases to the Environment from Facilities that Produce, Process, or Use Boron Trifluoride ${ }^{a}$}

\begin{tabular}{|c|c|c|c|c|c|c|c|c|c|}
\hline \multirow[b]{3}{*}{ State $^{c}$} & \multirow[b]{3}{*}{$\mathrm{RF}^{\mathrm{d}}$} & \multicolumn{8}{|c|}{ Reported amounts released in pounds per year ${ }^{b}$} \\
\hline & & \multirow[b]{2}{*}{ Air $^{\mathrm{e}}$} & \multirow[b]{2}{*}{ Water ${ }^{\mathrm{f}}$} & \multirow[b]{2}{*}{$\mathrm{UI}^{\mathrm{g}}$} & \multirow[b]{2}{*}{ Land $^{\text {h }}$} & \multirow[b]{2}{*}{ Other $^{i}$} & \multicolumn{3}{|c|}{ Total release } \\
\hline & & & & & & & On-site & Off-site ${ }^{k}$ & On- and off-site \\
\hline AR & 1 & 10 & 0 & 0 & 0 & 0 & 10 & 0 & 10 \\
\hline DE & 1 & 500 & 0 & 0 & 0 & 3,770 & 500 & 3,770 & 4,270 \\
\hline $\mathrm{FL}$ & 1 & 0 & 0 & 0 & 0 & 0 & 0 & 0 & 0 \\
\hline LA & 3 & 6 & 0 & 0 & 0 & 0 & 6 & 0 & 6 \\
\hline MD & 1 & 55 & 0 & 0 & 0 & 0 & 55 & 0 & 55 \\
\hline $\mathrm{NJ}$ & 1 & 0 & 0 & 0 & 0 & 0 & 0 & 0 & 0 \\
\hline OK & 1 & 1,750 & 0 & 0 & 0 & 0 & 1,750 & 0 & 1,750 \\
\hline PA & 3 & 0 & 0 & 0 & 0 & 0 & 0 & 0 & 0 \\
\hline SC & 2 & 11 & 0 & 0 & 4,983 & 0 & 11 & 4,983 & 4,994 \\
\hline TX & 7 & 2,306 & 0 & 0 & 0 & 0 & 2,306 & 0 & 2,306 \\
\hline Total & 21 & 4,638 & 0 & 0 & 4,983 & 3,770 & 4,638 & 8,753 & 13,391 \\
\hline
\end{tabular}

${ }^{a}$ The TRI data should be used with caution since only certain types of facilities are required to report. This is not an exhaustive list. Data are rounded to nearest whole number.

${ }^{\mathrm{b}}$ Data in TRI are maximum amounts released by each facility.

${ }^{\mathrm{c}}$ Post office state abbreviations are used.

${ }^{\mathrm{d}}$ Number of reporting facilities.

${ }^{\mathrm{e}}$ The sum of fugitive and point source releases are included in releases to air by a given facility.

${ }^{\mathrm{f}}$ Surface water discharges, waste water treatment-(metals only), and publicly owned treatment works (POTWs) (metal and metal compounds).

${ }^{\mathrm{g}}$ Class I wells, Class II-V wells, and underground injection.

${ }^{\mathrm{h}}$ Resource Conservation and Recovery Act (RCRA) subtitle C landfills; other on-site landfills, land treatment, surface impoundments, other land disposal, other landfills.

'Storage only, solidification/stabilization (metals only), other off-site management, transfers to waste broker for disposal, unknown

${ }^{\mathrm{j}}$ The sum of all releases of the chemical to air, land, water, and underground injection wells.

kTotal amount of chemical transferred off-site, including to POTWs.

$\mathrm{RF}=$ reporting facilities; $\mathrm{UI}=$ underground injection

Source: TRI07 2009 (Data are from 2007) 


\section{Table 6-2. Releases to the Environment from Facilities that Produce, Process, or Use Boron Trichloride ${ }^{a}$}

\begin{tabular}{|c|c|c|c|c|c|c|c|c|c|}
\hline \multirow[b]{3}{*}{ State $^{c}$} & \multirow[b]{3}{*}{$\mathrm{RF}^{\mathrm{d}}$} & \multicolumn{8}{|c|}{ Reported amounts released in pounds per year ${ }^{b}$} \\
\hline & & \multirow[b]{2}{*}{ Air ${ }^{\mathrm{e}}$} & \multirow[b]{2}{*}{ Water ${ }^{f}$} & \multirow[b]{2}{*}{$\mathrm{UI}^{\mathrm{g}}$} & \multirow[b]{2}{*}{ Land $^{\mathrm{h}}$} & \multirow[b]{2}{*}{ Other ${ }^{\mathrm{i}}$} & \multicolumn{3}{|c|}{ Total release } \\
\hline & & & & & & & On-site $^{j}$ & Off-site $^{k}$ & On- and off-site \\
\hline$\overline{\mathrm{CA}}$ & 1 & 0 & 0 & 0 & 0 & 0 & 0 & 0 & 0 \\
\hline MA & 1 & 0 & 0 & 0 & 0 & 0 & 0 & 0 & 0 \\
\hline NV & 1 & 0 & 0 & 0 & 0 & 0 & 0 & 0 & 0 \\
\hline $\mathrm{OH}$ & 1 & 0 & 0 & 0 & 0 & 0 & 0 & 0 & 0 \\
\hline PA & 1 & 0 & 0 & 0 & 0 & 0 & 0 & 0 & 0 \\
\hline SC & 1 & 35 & 0 & 0 & 2,422 & 0 & 2,422 & 35 & 2,422 \\
\hline WI & 1 & 35 & 0 & 0 & 0 & 0 & 0 & 35 & 0 \\
\hline WV & 1 & 0 & 0 & 0 & 0 & 0 & 0 & 0 & 0 \\
\hline Total & 8 & 70 & 0 & 0 & 2,422 & 0 & 2,422 & 70 & 2,422 \\
\hline
\end{tabular}

${ }^{a}$ The TRI data should be used with caution since only certain types of facilities are required to report. This is not an exhaustive list. Data are rounded to nearest whole number.

${ }^{\mathrm{b}}$ Data in TRI are maximum amounts released by each facility.

${ }^{\mathrm{C}}$ Post office state abbreviations are used.

${ }^{\mathrm{d}}$ Number of reporting facilities.

${ }^{\mathrm{e}}$ The sum of fugitive and point source releases are included in releases to air by a given facility.

'Surface water discharges, waste water treatment-(metals only), and publicly owned treatment works (POTWs) (metal and metal compounds).

${ }^{\mathrm{g}}$ Class I wells, Class II-V wells, and underground injection.

${ }^{\mathrm{h}}$ Resource Conservation and Recovery Act (RCRA) subtitle C landfills; other on-site landfills, land treatment, surface impoundments, other land disposal, other landfills.

iStorage only, solidification/stabilization (metals only), other off-site management, transfers to waste broker for disposal, unknown

${ }^{\mathrm{j}}$ The sum of all releases of the chemical to air, land, water, and underground injection wells.

${ }^{\mathrm{k}}$ Total amount of chemical transferred off-site, including to POTWs.

$\mathrm{RF}=$ reporting facilities; $\mathrm{UI}=$ underground injection

Source: TRI07 2009 (Data are from 2007) 
facilities producing fiberglass and other products (EPA 1987; Graedel 1978; Hollis et al. 1988; Lang et al. 1986; Rope et al. 1988; Stokinger 1981).

Boron or boron compounds have been identified in 1 air sample collected from 1,689 current or former NPL hazardous waste sites where it was detected in some environmental media (HazDat 2007).

\subsubsection{Water}

No releases of boron trifluoride or boron trichloride to surface water were reported from 21 and 5 domestic manufacturing and processing facilities, respectively, in 2006 (TRI06 2008). Releases for boron trifluoride and boron trichloride are summarized in Tables 6-1 and 6-2, respectively. There is no information on releases of other boron compounds to surface water from manufacturing and processing facilities because these releases are not required to be reported (EPA 1997).

Boron compounds are released to water in municipal sewage from perborates in detergents and in waste waters from coal-burning power plants, copper smelters, and industries using boron. Borate levels above background may be present in runoff waters from areas where boron-containing fertilizers or herbicides were used (Butterwick et al. 1989; Nolte 1988; Waggott 1969). An average boron concentration of $1 \mathrm{mg} / \mathrm{L}$ was reported in sewage effluents in California (Butterwick et al. 1989). Waggott (1969) reported that boron concentrations in municipal sewage in a treatment plant in England ranged from 2.5 to $6.5 \mathrm{mg} / \mathrm{L}$, releasing between 130 and $240 \mathrm{~kg}$ boron/day. Matthijs et al. (1999) reported boron concentrations of $0.41-1.2,0.39-0.96$, and $0.44-1.0 \mathrm{mg} / \mathrm{L}$ in raw sewage, settled sewage, and effluent, respectively, collected in 1994 from seven Dutch sewage treatment plants. These data demonstrate that boron passes through the sewage treatment process virtually unchanged. Since boron cannot be degraded and is not substantially absorbed during processing, there is almost no removal during the sewage treatment process (Fox et al. 2002).

Boron is also found in water produced from coal bed natural gas produced water (Jackson and Reddy 2007), irrigation, geothermal waste water, and thermal springs (Koç 2007) used for power generation and heating. In the Powder River Basin, Wyoming, boron was detected in coal natural gas waste water outfall and disposal ponds at 5.89-13.08 (0.064-0.14) and 7.72-15.20 $\mu \mathrm{M}(0.083-0.16 \mathrm{mg} / \mathrm{L})$, respectively (Jackson and Reddy 2007). In the Great Menderes Basin, Turkey, boron is brought to the surface and released from natural borate mineral deposits into local rivers via geothermal waste waters and crop 
irrigation canals (Koç 2007). The hot waste waters of a geothermal power plant released into the Buyuk Menderes River contained approximately 25-30 mg/L boron (Akar 2007).

Boron or boron compounds have been identified in 100 groundwater and 57 surface water samples collected from 1,689 NPL hazardous waste sites, where it was detected in some environmental media (HazDat 2007).

\subsubsection{Soil}

Estimated releases of 1,300 pounds ( 0.59 metric tons) of boron trifluoride to soil and 6 pounds (3 kilograms) by underground injection from 21 domestic manufacturing and processing facilities in 2006, accounted for about $100 \%$ of the estimated total environmental releases from facilities required to report to the TRI (TRI06 2008). Estimated releases of 136 pounds (61.7 kg) of boron trichlorideto soil and 18 pounds (8.2 kilograms) by underground injection from five domestic manufacturing and processing facilities in 2006, accounted for about $100 \%$ of the estimated total environmental releases from facilities required to report to the TRI (TRI06 2008). These releases for boron trifluoride and boron trichloride are summarized in Tables 6-1 and 6-2, respectively. There is no information on releases of other boron compounds to soil or by underground injection from manufacturing and processing facilities because these releases are not required to be reported (EPA 1997).

Boron is naturally released to soil and water by rainfall, weathering of boron-containing minerals, desorption from clays, and decomposition of boron-containing organic matter. Human-made sources include application of boron-containing fertilizers or herbicides, application of fly ash or sewage sludge as a soil amendment, the use of waste water for irrigation, or land disposal of boron-containing industrial wastes (Butterwick et al. 1989; Hollis et al. 1988; Mumma et al. 1984; Nolte 1988; Rope et al. 1988). In the Great Menderes Basin, Turkey, the hot waste waters of a geothermal power plant contained high levels of boron ( 25-30 mg/L). This water becomes part of the irrigation systems and is thus deposited in sediments and soils. The boron concentrations of surrounding irrigated soils before the waste water discharges began were $0.15 \mathrm{ppm}$ (0-20 cm depth) and 0.19 ppm (80-100 cm depth), while after the discharges, the boron concentrations were $13.90 \mathrm{ppm}$ (0-20 cm depth) and $3.66 \mathrm{ppm}$ (80-100 cm depth) (Akar 2007). Barber et al. (2006) reported that daily loads of boron in the Boulder Creek, Colorado of 0$55 \mathrm{~kg} /$ day are mostly due to the effluent of the Boulder waste water treatment plant. Mumma et al. (1984) reported that the boron concentration in sewage sludges from 23 U.S. cities ranged from 7.1 to $53.3 \mathrm{mg} / \mathrm{kg}$. Landfilling or land application is a common disposal method for these sludges. 
Boron or boron compounds have been identified in 37 soil and 21 sediment samples collected from 1,689 NPL hazardous waste sites, where it was detected in some environmental media (HazDat 2007).

\subsection{ENVIRONMENTAL FATE}

\subsubsection{Transport and Partitioning}

Boron is generally found in nature bound to oxygen and is never found as the free element (Cotton et al. 1999). Atmospheric boron may be in the form of particulate matter or aerosols as borides, boron oxides, borates, boranes, organoboron compounds, trihalide boron compounds, or borazines. Borates are relatively soluble in water, and will probably be removed from the atmosphere by precipitation and dry deposition (EPA 1987). The half-life of airborne particles is usually on the order of days, depending on the size of the particle and atmospheric conditions (Nriagu 1979). No specific information on the fate of atmospheric boron was located.

Boron readily hydrolyzes in water to form the electrically neutral, weak monobasic acid, boric acid $\left(\mathrm{H}_{3} \mathrm{BO}_{3}\right)$, and the monovalent ion, $\mathrm{B}(\mathrm{OH})_{4}{ }^{-}$. In concentrated solutions, boron may polymerize, leading to the formation of complex and diverse molecular arrangements. Rai et al. (1986) concluded that because most environmentally relevant boron minerals are highly soluble in water, it is unlikely that mineral equilibria will control the fate of boron in water. Boron was found to not be significantly removed during the conventional treatment of waste water (Matthijs et al. 1999; Pahl et al. 2001; Waggott 1969). Boron may, however, be co-precipitated with aluminum, silicon, or iron to form hydroxyborate compounds on the surfaces of minerals (Biggar and Fireman 1960).

Waterborne boron may be adsorbed by soils and sediments. Adsorption-desorption reactions are expected to be the only significant mechanism that will influence the fate of boron in water (Rai et al. 1986). The extent of boron adsorption depends on the $\mathrm{pH}$ of the water and the chemical composition of the soil. The greatest adsorption is generally observed at pH 7.5-9.0 (Keren and Mezuman 1981; Keren et al. 1981; Waggott 1969). Bingham et al. (1971) concluded that the single most important property of soil that will influence the mobility of boron is the abundance of amorphous aluminum oxide. The extent of boron adsorption has also been attributed to the levels of iron oxide (Sakata 1987), and to a lesser extent, the organic matter present in the soil (Parks and White 1952), although other studies (Mezuman and Keren 1981) found that the amount of organic matter present was not important. 
The adsorption of boron may not be reversible in some soils. The lack of reversibility may be the result of solid-phase formation on mineral surfaces (Rai et al. 1986) and/or the slow release of boron by diffusion from the interior of clay minerals (Griffin and Burau 1974).

Partition coefficients such as adsorption constants describe the tendency of a chemical to partition from water to solid phases. Adsorption constants for inorganic constituents such as boron cannot be predicted a priori, but must be measured for each soil-water combination. Compilations of available data for boron are given elsewhere (Rai et al. 1986). In general, boron adsorption will be most significant in soils that contain high concentrations of amorphous aluminum and iron oxides and hydroxides such as the reddish Ultisols in the southeastern United States.

It is unlikely that boron is bioconcentrated significantly by organisms from water. A bioconcentration factor (BCF) relates the concentration of a chemical in the tissues of aquatic and terrestrial animals or plants to the concentration of the chemical in water or soil. The BCFs of boron in marine and freshwater plants, fish, and invertebrates were estimated to be $<100$ (Thompson et al. 1972). Experimentally measured BCFs for fish have ranged from 52 to 198 (Tsui and McCart 1981). These BCFs suggest that boron is not significantly bioconcentrated.

\subsubsection{Transformation and Degradation}

\subsubsection{Air}

There is no information available that suggests that particulate boron compounds are transformed or degraded in the atmosphere. Particulate-phase boron compounds would be removed from the atmosphere by wet and dry deposition. Volatile boron trihalides are moisture sensitive and will hydrolyze to boric acid and their corresponding halogen acid (Culver et al. 1994b).

\subsubsection{Water}

As an element, boron itself cannot be degraded in the environment; however, it may undergo various reactions that change the form of boron (e.g., precipitation, polymerization, and acid-base reactions) depending on conditions such as its concentration in water and $\mathrm{pH}$. In nature, boron is generally found in its oxygenated form (Cotton et al. 1999). In aqueous solution, boron is normally present as boric acid and 
borate ions, with the dominant form of inorganic boron in natural aqueous systems as undissociated boric acid (Choi and Chen 1979). Boric acid acts as an electron acceptor in aqueous solution, accepting an hydroxide ion from water to form $\left(\mathrm{B}(\mathrm{OH})_{4}\right)^{-}$ion. In dilute solution, the favored form of boron is $\mathrm{B}(\mathrm{OH})_{4}{ }^{-}$ (Cotton et al. 1999). In more concentrated solutions ( $>0.1 \mathrm{M}$ boric acid) and at neutral to alkaline $\mathrm{pH}$ (611), polymeric species are formed (e.g., $\mathrm{B}_{3} \mathrm{O}_{3}(\mathrm{OH})_{4}{ }_{4}^{-}, \mathrm{B}_{5} \mathrm{O}_{6}(\mathrm{OH})_{4}{ }^{-}, \mathrm{B}_{3} \mathrm{O}_{3}(\mathrm{OH})_{5}{ }^{2-}$, and $\left.\mathrm{B}_{4} \mathrm{O}_{5}(\mathrm{OH})_{4}{ }^{2-}\right)(\mathrm{Choi}$ and Chen 1979; Cotton et al. 1999).

\subsubsection{Sediment and Soil}

Most boron compounds are transformed to borates in soil due to the presence of moisture. Borates themselves are not further degraded in soil. However, borates can exist in a variety of forms in soil (see Section 6.2.3). Borates are removed from soils by water leaching and by assimilation by plants.

\subsection{LEVELS MONITORED OR ESTIMATED IN THE ENVIRONMENT}

Reliable evaluation of the potential for human exposure to boron depends in part on the reliability of supporting analytical data from environmental samples and biological specimens. Concentrations of boron in unpolluted atmospheres and in pristine surface waters are often so low as to be near the limits of current analytical methods. In reviewing data on boron levels monitored or estimated in the environment, it should also be noted that the amount of chemical identified analytically is not necessarily equivalent to the amount that is bioavailable. The analytical methods available for monitoring boron in a variety of environmental media are detailed in Chapter 7.

\subsubsection{Air}

Boron concentrations in ambient air samples have been reported to range from $<5 \times 10^{-7}$ to $8 \times 10^{-5} \mathrm{mg} / \mathrm{m}^{3}$, with an average concentration of $2 \times 10^{-5} \mathrm{mg} / \mathrm{m}^{3}$ (Howe 1998). Bertine and Goldberg (1971) estimated that approximately 11,600 tons of boron are injected into the atmosphere as a component of fly ash produced by coal combustion, which was estimated to contain an average boron concentration of about $75 \mathrm{mg} / \mathrm{kg}$. Mean dust concentrations ranging from 3.3 to $18 \mathrm{mg}$ particulates $/ \mathrm{m}^{3}$ were measured in air samples from U.S. facilities where borax was packaged and shipped (Culver et al. 1994a). Dust samples in these facilities were predominantly composed of various types of borates and ranged from 11.8 to $15.2 \%$ boron by weight. 


\subsubsection{Water}

Boron is widely distributed in surface water and groundwater. The average surface water boron concentration in the United States is about $0.1 \mathrm{mg} / \mathrm{L}$ (Butterwick et al. 1989; EPA 1986b), but concentrations vary greatly, depending on boron content of local geologic formations and anthropogenic sources of boron (Butterwick et al. 1989). A survey of U.S. surface waters detected boron in $98 \%$ of 1,577 samples at concentrations ranging from 0.001 to $5 \mathrm{mg} / \mathrm{L}$. Mean boron concentrations calculated for the 15 drainage basins in the continental United States ranged from $0.019 \mathrm{mg} / \mathrm{L}$ in the Western Great Lakes Basin to $0.289 \mathrm{mg} / \mathrm{L}$ in the Western Gulf Basin (Butterwick et al. 1989). Mean boron concentration ranging from 0.28 to $7.8 \mathrm{mg} / \mathrm{L}$ were reported in samples collected during 1985-2002 from 26 sites in the San Joaquin River, California (Hall et al. 2004). The concentration of boron in sea water is about 4.5 mg/L (Butterwick et al. 1989; EPA 1986b).

Several studies have measured boron concentrations in water in those areas of California with boron-rich deposits. Reported high boron concentrations in surface waters ranged from $15 \mathrm{mg} / \mathrm{L}$ in coastal drainage waters to $360 \mathrm{mg} / \mathrm{L}$ in a boron-rich lake (Butterwick et al. 1989; Deverel and Millard 1988). Mean boron concentration in a California river ranged from 0.30 to $0.50 \mathrm{mg} / \mathrm{L}$ over a 20 -year period (Butterwick et al. 1989). Reported boron concentrations in groundwater in the San Joaquin Valley ranged from 0.14 to $120 \mathrm{mg} / \mathrm{L}$ with a median concentration of about $4 \mathrm{mg} / \mathrm{L}$ (Butterwick et al. 1989; Deverel and Millard 1988). Waggott (1969) reported that groundwater boron concentrations $>100 \mathrm{mg} / \mathrm{L}$ are common in California.

Drinking water surveys generally do not report boron concentration. Concentrations of boron in tap water have been reported in a range of 0.007-0.2 mg/L in the United States and England (Choi and Chen 1979; Waggott 1969), and the National Inorganics and Radionuclides Survey completed in 1987 reported relatively widespread occurrence of boron in 989 public water supplies (NIRS 1987). Boron concentrations ranged from $<0.005$ to $>2 \mathrm{mg} / \mathrm{L}$, with concentrations of up to $0.4 \mathrm{mg} / \mathrm{L}$ in $90 \%$ of systems (NIRS 1987). A survey of 969 public water supply systems showed that $99 \%$ contained boron at $<1 \mathrm{mg} / \mathrm{L}$. The maximum level measured was $3.28 \mathrm{mg} / \mathrm{L}$ (McCabe et al. 1970). Davies (1990) reported an average boron concentration of $0.0258 \mathrm{mg} / \mathrm{L}$ in drinking water from Toronto, Canada (1978-1984).

\subsubsection{Sediment and Soil}

Background boron levels in U.S. soils were reported at a geometric mean concentration of $26 \mathrm{mg} / \mathrm{kg}$ with a maximum concentration of $300 \mathrm{mg} / \mathrm{kg}$ (Eckel and Langley 1988). Similar concentrations were reported 
in a U.S. Geological Survey report (USGS 1984), with an average boron concentration of $33 \mathrm{mg} / \mathrm{kg}$ (range $<20-300 \mathrm{mg} / \mathrm{kg}$ ) in surface soils from the conterminous United States. Mean boron concentrations in soil collected in the summer of 1981 from the Idaho National Engineering Laboratory and a reference site were 10.1 and $4.7 \mathrm{mg} / \mathrm{kg}$ dry weight, respectively (Rope et al. 1988). A geometric mean boron concentration of $8.98 \mathrm{mg} / \mathrm{kg}$ (range $2.90-38.0 \mathrm{mg} / \mathrm{kg}$ ) was reported in soil collected from Aviles, northern Spain (Ordonez et al. 2003).

Boron is an essential nutrient for plants. Boron soil concentrations for optimum plant growth reportedly range from 0.1 to $0.5 \mathrm{mg} / \mathrm{kg}$ for several plant species (Butterwick et al. 1989).

Geometric mean boron concentration in sediment collected in 1993 and 1994 from 16 Great Lake embayments and riverine environments of eastern Lake Erie, southern Lake Ontario, and the Niagara River ranged from 0.5 to $7.9 \mathrm{mg} / \mathrm{kg}$ dry weight (Lowe and Day 2002). Boron concentrations in sediments collected in 1992 from the Neosho River Basin in Kansas ranged from 2 to 6.5 mg/kg dry weight (Allen et al. 2001).

\subsubsection{Other Environmental Media}

Boron concentrations in various foods are summarized in Table 6-3. Rainey et al. $(1999,2002)$ reported the highest content of boron in foods such as raisins, peanut butter, peanuts, dried fruits, and avocados with concentrations of 2.20, 1.45, 1.70, 1.87, and $1.22 \mathrm{mg} / 100 \mathrm{~g}$ food, respectively. The top two items that contribute to boron intake were coffee and milk, due to the volume with which they are consumed (Rainey et al. 1999, 2002). Hunt et al. (1991) determined boron concentrations various foods. In general, boron concentrations were lowest in foods such as meats, cereal and grain products, and confections, ranging from $\leq 0.015 \mathrm{mg} / \mathrm{kg}$ in many of these foods to $1.470 \mathrm{mg} / \mathrm{kg}$ in grape jelly. Fruits, vegetables, herbs, and spices contained the highest concentrations of boron, including parsley flakes (26.878 mg/kg), ground cinnamon (10.370 mg/kg); dried onion flakes (6.573 mg/kg), and applesauce (2.828 mg/kg). Meacham and Hunt (1994) studied the boron content in infant (6-11 months) foods. Foods containing fruit typically had the highest concentrations of boron: prunes with tapioca (2.6 mg/kg); apples with ham (2.5 mg/kg); applesauce with apricot (2.5 mg/kg); pears (1.9 mg/kg); and applesauce (1.8 mg/kg). Boron 
Table 6-3. Boron Levels in Food

\begin{tabular}{|c|c|}
\hline Food item & Level $(\mu \mathrm{g} / 100 \mathrm{~g})^{a}$ \\
\hline \multicolumn{2}{|l|}{ Fruits and vegetables } \\
\hline Apples, raw & 360 \\
\hline Applesauce, unsweetened & 280 \\
\hline Avacado, guacamole & $1,222^{b}$ \\
\hline Bananas, raw & 135 \\
\hline Beans, string, cooked & 120 \\
\hline Broccoli, boiled & 250 \\
\hline Cantaloupe, raw & 180 \\
\hline Carrots, raw or frozen & 140 \\
\hline Coleslaw with dressing & 120 \\
\hline Corn, yellow, cooked & 46 \\
\hline Fruit cocktail, canned in heavy syrup & 240 \\
\hline Fruit, dried & 1,870 \\
\hline Grapes, raw & 490 \\
\hline Lettuce, raw & 105 \\
\hline Onions, raw & 190 \\
\hline Oranges, raw & 260 \\
\hline Peaches, raw & 530 \\
\hline Pears, raw & 280 \\
\hline Peas, green, cooked & 130 \\
\hline Potatoes, not fried & $62^{\mathrm{b}}$ \\
\hline Raisins & 2,200 \\
\hline Spinach, boiled & 180 \\
\hline Tomatoes, raw & 63 \\
\hline \multicolumn{2}{|l|}{ Beverages } \\
\hline Apple juice & 180 \\
\hline Beer & 12 \\
\hline Coffee, from ground beans & 29 \\
\hline Fruit-flavored drink from powder & 16 \\
\hline Grape juice unsweetened & 300 \\
\hline Milk, whole & 18 \\
\hline Orange juice & 72 \\
\hline Soft drink, cola-type & 13 \\
\hline Tea, leaf, brewed & 9 \\
\hline Wine, table, dry & 610 \\
\hline
\end{tabular}


Table 6-3. Boron Levels in Food

\begin{tabular}{|c|c|}
\hline Food item & Level $(\mu \mathrm{g} / 100 \mathrm{~g})^{\mathrm{a}}$ \\
\hline \multicolumn{2}{|l|}{ Meat/fish products } \\
\hline Beef and vegetable stew & 120 \\
\hline Beef, burgers & $23^{b}$ \\
\hline Beef vegetable soup with potato, stew type & 140 \\
\hline Chicken breast, broiled, without skin & 27 \\
\hline Chili con carne, with beans & 170 \\
\hline Hamburger, with tomato and/or ketchup & 51 \\
\hline Tuna, canned, water packed & 54 \\
\hline \multicolumn{2}{|l|}{ Other } \\
\hline Beans, lima, dry cooked, fat added & 370 \\
\hline Beans, refried & 400 \\
\hline Bran flakes with raisins & 450 \\
\hline Bread, white & 46 \\
\hline Cakes & $100^{\mathrm{b}}$ \\
\hline Cereal, ready to eat & $128^{\mathrm{b}}$ \\
\hline Cookies, brownies & $112^{\mathrm{b}}$ \\
\hline French fries, from frozen, deep fried & 110 \\
\hline Donuts, sweet rolls, etc. & 69 \\
\hline Ice cream, regular, not chocolate & 22 \\
\hline Nuts/seeds, whole & $1214^{\mathrm{b}}$ \\
\hline Pasta & $44^{\mathrm{b}}$ \\
\hline Peanut butter & 1,450 \\
\hline Peanuts, roasted, salted & 1,700 \\
\hline Peas, black-eyed, cooked, fat added & 65 \\
\hline Pizza with meat, thin crust & 490 \\
\hline Potato chips & 325 \\
\hline Rice, white, cooked & 32 \\
\hline Spaghetti sauce & 120 \\
\hline Spaghetti with meat sauce & 65 \\
\hline Tomato sauce & $132^{\mathrm{b}}$ \\
\hline
\end{tabular}

${ }^{\text {a}}$ Rainey et al. 1999 (CSFII 1989-1991), unless otherwise noted.

${ }^{b}$ Rainey et al. 2002 (CSFII 1994-1996) 
is also found in infant formula $(120 \mu \mathrm{g} / \mathrm{L})$ and expressed human breast milk $(10-100 \mu \mathrm{g} / \mathrm{L})$ (Hunt and Meacham 2001; Hunt et al. 2004, 2005; Lopez-Garcia et al. 2009).

Minoia et al. (1994) determined the concentrations of various elements in beverages available in Italy. Mean boron concentrations in wine, mineral water, beer, ready-to-drink-infusion of tea, and instant coffees were $1.164,0.112,0.166,0.219$, and $0.085 \mathrm{mg} / \mathrm{L}$, respectively. In this study, it was estimated that beverages contributed 34\% to the estimated weekly total dietary intake of $9 \mathrm{mg}$ of boron (Minoia et al. 1994). Red and white Australian wine samples that were analyzed contained boron concentrations of approximately $2.5 \mathrm{mg} / \mathrm{L}$ (Cozzolino et al. 2008).

Boric acid, anhydrous sodium tetraborate, and sodium tetraborate decahydrate (borax) are found in various commercial products including pesticides, plant foods, household cleaners, laundry detergents, facial creams and cleaners, shampoo, diaper rash ointments, and pet products. Typical amounts of borax in detergents range from 1 to 5\%. Boric acid concentrations in various ant and roach pesticide products range from 5 to $100 \%$ (NIH 2004).

Gonzales et al. (2004) determined elements found in dust collected from homes of Native Americans in Zuni Pueblo, New Mexico where jewelry was produced. Surface dust samples were collected from work and living areas of jewelers' homes and from control homes. A surface area of $715 \mathrm{~cm}^{2}$ was wiped at each location. Mean boron concentrations were found to be significantly higher in work areas ( $0.87 \mu \mathrm{g} / \mathrm{sample})$ than in living areas $(0.28 \mu \mathrm{g} / \mathrm{sample})$ of homes where jewelry was made. The geometric mean boron concentration was $0.19 \mu \mathrm{g} /$ sample in living areas of homes in which no jewelry was made (Gonzales et al. 2004).

The geometric mean boron concentration in soft tissues of zebra mussels collected in 1993 and 1994 from 16 Great Lake embayments and riverine environments of eastern Lake Erie, southern Lake Ontario, and the Niagara River ranged from 0.92 to $6.89 \mu \mathrm{g} / \mathrm{g}$ dry weight (Lowe and Day 2002). Boron was not detected (detection limit 2-4 $\mu \mathrm{g} / \mathrm{g}$ dry weight) in the soft tissues of mussels collected in 1991 or in fish composites collected in 1990-1992 from the Neosho River Basin in Kansas (Allen et al. 2001).

\subsection{GENERAL POPULATION AND OCCUPATIONAL EXPOSURE}

Human exposure to borates may occur through ingestion of food and water or insecticides used to control cockroaches, inhalation of boron-containing powders or dusts, or the use of boron from cosmetics or 
medical preparations. The most appreciable boron exposure to the general population is likely to be ingestion of food and to a lesser extent in water (Beyer et al. 1983; Waggott 1969). As boron is a natural component of the environment, individuals will have some exposure from foods and drinking water.

Dietary intakes of boron in children and adults in the United States are summarized in Table 6-4. Rainey et al. (1999) reported mean daily intakes of boron for male and female adults to be 1.17 and $0.96 \mathrm{mg} / \mathrm{day}$, respectively (range $0.02->9 \mathrm{mg} /$ day). The highest median boron intake of $1.30 \mathrm{mg} /$ day was found for adult male vegetarians, and the lowest median boron intake of $0.72 \mathrm{mg} /$ day was found for women aged 19-30 years. Median boron intakes were higher in adult male and female vegetarians, 1.47 and $1.29 \mathrm{mg} /$ day, respectively, than for all adult males and females, 1.17 and $0.96 \mathrm{mg} /$ day, respectively (Rainey et al. 1999). Consumption of fruits and vegetables contribute largely to boron intake in the human diet. An average daily intake of $1 \mathrm{mg}$ was reported for boron for individuals in the United States. Consumption of wine may contribute an additional 3-4 mg/day of boron (Pahl et al. 2001).

Concentrations of various elements were determined in hair samples from women in two areas (acid and alkaline) of southern Sweden (Rosborg et al. 2003). Median boron concentrations were 281 and $<1 \mathrm{mg} / \mathrm{kg}$ in hair samples from the acid and alkaline areas, respectively. In this study, the boron levels in drinking water were similar, 10.6 and $9.3 \mu \mathrm{g} / \mathrm{L}$, in the acid and alkaline areas, respectively, and the authors noted that drinking water did not explain the significantly higher concentrations of boron in the individuals living in the acid area (Rosborg et al. 2003). A mean boron concentration of $0.50 \mathrm{mg} / \mathrm{kg}$ wet weight was reported in lung tissue collected from 26 nonsmoking individuals, aged $>50$ years with no history of occupational exposure to elements living in Terni, central Italy (Alimonti et al. 1992). Boron was not detected in a national survey of human adipose tissue (EPA 1986a).

Occupational exposure to boron compounds may be higher. Workers in other industries, including manufacture of glass wool (Jensen 2009), fiberglass and other glass products, cleaning and laundry products, fertilizers, pesticides, and cosmetics, may also be exposed to boron compounds (Stokinger 1981). Culver et al. (1994a) reported average end-of-shift boron concentrations in blood and urine of 0.11-0.26 $\mu \mathrm{g} / \mathrm{g}$ and 3.16-10.72 $\mu \mathrm{g} / \mathrm{mg}$ creatinine, respectively, collected from workers at a facility where borax is packaged and shipped. Average boron concentrations in blood and urine collected Monday morning prior to the first shift of the week were $0.09 \mu \mathrm{g} / \mathrm{g}$ and $2.75 \mu \mathrm{g} / \mathrm{mg}$ creatinine, respectively (Culver et al. 1994a). In 2004, workers from Kuandian City, China with occupations in boron mines or processing plants producing borax or boric acid were monitored over a 24-hour period for boron 
Table 6-4. Dietary Boron Intake

\begin{tabular}{|c|c|c|}
\hline & $1989-1991^{a}$ & $1994-1996^{b}$ \\
\hline Age group & \multicolumn{2}{|c|}{ Mean \pm standard deviation (mg/day) } \\
\hline \multicolumn{3}{|l|}{ School aged male and female children } \\
\hline $4-8$ years $\left(n=993^{a}, 1,650^{b}\right)$ & $0.85 \pm 0.040$ & $0.80 \pm 0.01$ \\
\hline $9-13$ years $\left(n=943^{a}, 552^{b}\right.$ males $)$ & $0.91 \pm 0.45$ & $0.90 \pm 0.03(\mathrm{~m})$ \\
\hline$\left(\mathrm{n}=560^{\mathrm{b}}\right.$ females) & & $0.83 \pm 0.03(f)$ \\
\hline $14-18$ years $\left(n=759^{a}, 446^{b}\right.$ males $)$ & $0.88 \pm 0.47$ & $1.02 \pm 0.04(\mathrm{~m})$ \\
\hline$\left(\mathrm{n}=436^{\mathrm{b}}\right.$, females $)$ & & $0.078 \pm 0.04(f)$ \\
\hline Adult males $(\geq 19$ years $)\left(n=3,433^{a}, 4,817^{b}\right)$ & $1.17 \pm 0.65$ & $1.28 \pm 0.02$ \\
\hline $19-30$ years $\left(n=878^{a}, 853^{b}\right)$ & $1.07 \pm 0.64$ & $1.15 \pm 0.03$ \\
\hline $31-50$ years $\left(n=1,297^{a}, 1,684^{b}\right)$ & $1.17 \pm 0.64$ & $1.33 \pm 0.03$ \\
\hline $51-70$ years $\left(n=884^{a}, 1,606^{b}\right)$ & $1.28 \pm 0.67$ & $1.34 \pm 0.02$ \\
\hline$>70$ years $\left(n=374^{a}, 674^{b}\right)$ & $1.19 \pm 0.61$ & $1.25 \pm 0.03$ \\
\hline Vegetarian $\left(n=49^{\mathrm{a}}\right)$ & $1.47 \pm 0.70$ & No data \\
\hline Adult females ( $\geq 19$ years $)\left(n=4,881^{a}, 4,536^{b}\right)$ & $0.96 \pm 0.55$ & $1.00 \pm 0.01$ \\
\hline $19-30$ years $\left(n=1,199^{a}, 760^{b}\right)$ & $0.86 \pm 0.55$ & $0.87 \pm 0.03$ \\
\hline $31-50$ years $\left(n=1,734^{a}, 1,614^{b}\right)$ & $0.96 \pm 0.55$ & $1.00 \pm 0.02$ \\
\hline $51-70$ years $\left(n=1,220^{a}, 1,539^{b}\right)$ & $1.05 \pm 0.55$ & $1.11 \pm 0.02$ \\
\hline$>70$ years $\left(n=728^{a}, 623^{b}\right)$ & $0.97 \pm 0.52$ & $0.98 \pm 0.03$ \\
\hline Vegetarian $\left(n=130^{a}\right)$ & $1.29 \pm 1.12$ & No data \\
\hline Pregnant women $\left(n=130^{a}, 70^{b}\right)$ & $1.01 \pm 0.72$ & $1.16 \pm 0.09$ \\
\hline
\end{tabular}

${ }^{a}$ Rainey et al. 1999 (CSFII 1989-1991)

${ }^{\mathrm{b}}$ Rainey et al. 2002 (CSFII 1994-1996) 
exposure. Total daily boron measured in food, fluids, and personal air averaged $41.2 \mathrm{mg} /$ day for workers in the boron industry, $4.3 \mathrm{mg} /$ day in the surrounding community, and $2.3 \mathrm{mg} / \mathrm{day}$ for the comparison group. Blood, post-shift urine, and semen levels of boron determined for the boron industry workers were 19.3, 499.2, and $793.9 \mathrm{mg}$ /day, respectively while the comparison group only had 1.7, 48.0, and $215.0 \mathrm{mg} /$ day, respectively (Xing et al. 2008).

Workers in other industries, including manufacture of fiberglass and other glass products, cleaning and laundry products, fertilizers, pesticides, and cosmetics, may also be exposed to boron compounds (Stokinger 1981). Reported concentrations of borax dust in different areas of a large borax mining and refining plant ranged from 1.1 to $14.6 \mathrm{mg} / \mathrm{m}^{3}$ for total particulate (Garabrant et al. 1985) and the mean boric acid/boron oxide dust concentration in a boric acid manufacturing plant was $4.1 \mathrm{mg} / \mathrm{m}^{3}$ for total particulate (Garabrant et al. 1984). Mean dust concentrations ranging from 3.3 to $18 \mathrm{mg}$ particulates $/ \mathrm{m}^{3}$ were measured in air samples from U.S. facilities where borax was packaged and shipped (Culver et al. 1994a). Dust samples in these facilities were predominantly composed of various types of borates and ranged from 11.8 to $15.2 \%$ boron by weight. In another study of dust concentrations in air samples from a U.S. borax production facility, mean total dust concentrations ranged from 0.29 to $18.95 \mathrm{mg}$ particulates $/ \mathrm{m}^{3}$, with average percent boron contents in dust ranging from 5.6 to $10.1 \%$ (Woskie et al. 1994).

NIOSH estimated that the number of workers potentially exposed to boron increased from 6,500 in the early 1970s (NOHS 1989) to 35,600 in the early 1980s (NOES 1989). Neither the National Occupational Hazard Survey (NOHS) nor the National Occupational Exposure Survey (NOES) databases contain information on the frequency, concentration, or duration of exposures of workers to any of the chemicals listed therein. These surveys provide only estimates of the number of workers potentially exposed to chemicals in the workplace. Sittig (1985) reports that NIOSH estimated that the numbers of workers potentially exposed to borax, boron oxide, and boron trifluoride are 2,490,000, 21,000, and 50,000, respectively.

\subsection{EXPOSURES OF CHILDREN}

This section focuses on exposures from conception to maturity at 18 years in humans. Differences from adults in susceptibility to hazardous substances are discussed in Section 3.7, Children's Susceptibility. 
Children are not small adults. A child's exposure may differ from an adult's exposure in many ways. Children drink more fluids, eat more food, breathe more air per kilogram of body weight, and have a larger skin surface in proportion to their body volume. A child's diet often differs from that of adults. The developing human's source of nutrition changes with age: from placental nourishment to breast milk or formula to the diet of older children who eat more of certain types of foods than adults. A child's behavior and lifestyle also influence exposure. Children crawl on the floor, put things in their mouths, sometimes eat inappropriate things (such as dirt or paint chips), and spend more time outdoors. Children also are closer to the ground, and they do not use the judgment of adults to avoid hazards (NRC 1993).

Exposure to boron for children will be similar to adults and will occur primarily through the diet. As boron is a natural component of the environment, children, as with the general population, will have some exposure from foods and drinking water. Rainey et al. (2002) reported that average daily boron intakes ranged from 0.75 to $0.99 \mathrm{mg}$ in infants and children aged $0-8$ years. In adolescent males and females aged 9-18 years, average daily boron intakes were $0.9-1.02$ and $0.78-0.83 \mathrm{mg}$, respectively. A daily boron intake of $1.16 \mathrm{mg}$ was reported for pregnant women. Dietary intake of boron in children in the United States is summarized in Table 6-4. Meacham and Hunt (1994) reported a daily intake of $0.333 \mathrm{mg}$ for infants (6-11 months) from baby foods and beverages.

Children and infants may be exposed to boric acid or its sodium salts in homes where pesticide products containing boric acid or its sodium salts are used. Individuals applying these products in residential setting should take appropriate precautions to avoid exposing children.

\subsection{POPULATIONS WITH POTENTIALLY HIGH EXPOSURES}

The populations living in areas of California and other western states with boron-rich geological deposits have potentially high exposure to boron from drinking water and locally grown foods (Butterwick et al. 1989). Individuals using boron-containing cosmetics or medicines extensively, especially on damaged skin, may be exposed to higher-than-normal levels of boron (Beyer et al. 1983). Workers in industries producing or using boron-containing materials also have potentially high exposure as noted above (Section 6.5). People living in the vicinity of waste sites are also at risk of higher-than-normal exposure levels. 


\subsection{ADEQUACY OF THE DATABASE}

Section 104(i)(5) of CERCLA, as amended, directs the Administrator of ATSDR (in consultation with the Administrator of EPA and agencies and programs of the Public Health Service) to assess whether adequate information on the health effects of boron is available. Where adequate information is not available, ATSDR, in conjunction with NTP, is required to assure the initiation of a program of research designed to determine the health effects (and techniques for developing methods to determine such health effects) of boron.

The following categories of possible data needs have been identified by a joint team of scientists from ATSDR, NTP, and EPA. They are defined as substance-specific informational needs that if met would reduce the uncertainties of human health assessment. This definition should not be interpreted to mean that all data needs discussed in this section must be filled. In the future, the identified data needs will be evaluated and prioritized, and a substance-specific research agenda will be proposed.

\subsubsection{Identification of Data Needs}

Physical and Chemical Properties. Table 4-2 summarizes many of the relevant physical and chemical properties of boron and selected boron compounds. There are adequate data for the physical and chemical properties of boron and boron compounds. No data needs are identified.

Production, Import/Export, Use, Release, and Disposal. According to the Emergency Planning and Community Right-to-Know Act of 1986, 42 U.S.C. Section 11023, industries are required to submit substance release and off-site transfer information to the EPA. The TRI, which contains this information for 2006, became available in May of 2008. This database is updated yearly and should provide a list of industrial production facilities and emissions.

Current data on the production volume and uses of boron and boron compounds are available and no further production data are necessary at this time (Alam et al. 2003; USGS 2008, 2009); however, a data need exists for disposal methods of boron-containing wastes.

Environmental Fate. The only quantifiable mechanism that influences the fate of boron is soil adsorption (Rai et al. 1986). A data need exists for the adsorption and mobility of boron in soils low in aluminum oxide, since aluminum oxide content of soils is an important property of soil that will influence the mobility of boron (Bingham et al. 1971). 
Bioavailability from Environmental Media. Boron compounds can be absorbed following inhalation of contaminated workplace air, ingestion of contaminated food, or through damaged skin (Draize and Kelley 1959; Wong et al. 1964). The most significant routes of exposure near hazardous waste sites are likely to be through drinking boron-contaminated water and ingestion of locally grown food (Beyer et al. 1983; Butterwick et al. 1989). A data need exists for the amount of boron that is bioavailable from environmentally relevant media, such as drinking water, food, and soil.

Food Chain Bioaccumulation. Only one study was located where boron bioconcentration was actually measured (Tsui and McCart 1981). Future research may be helpful, but it appears that boron is not significantly bioconcentrated. There are no data on the biomagnification of boron in the food chain, but it is not likely that bioaccumulation is a major environmental concern. Therefore, there are no data needs at this time.

Exposure Levels in Environmental Media. Reliable monitoring data for the levels of boron in contaminated media at hazardous waste sites are needed so that the information obtained on levels of boron in the environment can be used in combination with the known body burden of boron to assess the potential risk of adverse health effects in populations living in the vicinity of hazardous waste sites.

Data on boron levels in surface water and soil are available (Butterwick et al. 1989; Eckel and Langley 1988; EPA 1986b; Hall et al. 2004; Ordonez et al. 2003; Rope et al. 1988; USGS 1984). Data on boron concentration in drinking water are limited (Choi and Chen 1979; Davies 1990; McCabe et al. 1970; NIRS 1987; Waggott 1969). Boron concentrations in foods and beverages have been reported (Hunt et al. 1991; Minoia et al. 1994; Rainey et al. 1999, 2002). Additional data on boron concentrations in air and food, and more recent data on boron concentrations in drinking water would be useful in estimating the exposure of humans to boron.

Exposure Levels in Humans. Background levels of boron in human blood, urine, and hair have been reported (Alimonti et al. 1992; Culver et al. 1994a; Rosborg et al. 2003; Stokinger 1981). Additional data on blood and/or urine concentrations in individuals with potentially high exposure to boron would be useful in assessing the magnitude of human exposure.

This information is necessary for assessing the need to conduct health studies on these populations. 
Exposures of Children. Children are exposed to boron by the same routes as adults. Rainey et al. (2002) reported daily boron intakes of 0.75 and $0.99 \mathrm{mg}$ for infants aged 0-6 and 7-11 months respectively. In children, 0.86 and $0.80 \mathrm{mg}$ daily boron intakes were reported for 1-3 and 4-8 year olds, respectively. In male adolescents, 0.90 and $1.02 \mathrm{mg}$ were reported for 9-13 and 14-18 year olds, respectively. In female adolescents, 0.83 and $0.78 \mathrm{mg}$ were reported for 9-13 and 14-18 year olds, respectively. Meacham and Hunt (1994) reported a daily intake of $0.333 \mathrm{mg}$ for infants (6-11 months) from baby foods and beverages. There do not appear to be any childhood-specific means to decrease exposure to boron.

Boron intake from infant food was estimated to supply $47 \%$ of the $0.55 \mathrm{mg}$ of daily boron to infants aged 6-11 months and toddlers aged 2 years $0.54 \mathrm{mg}$; 38\% from fruits and juices and 19\% from milk and cheese (Hunt and Meacham 2001). Children may also be exposed to boron by breastfeeding mothers. Boron has been detected in breast milk with concentrations ranging from 10 to $100 \mu \mathrm{g} / \mathrm{L}$, although higher levels have been found in infant formulas $(120 \mu \mathrm{g} / \mathrm{L})$ and whole cow milk $(280 \mu \mathrm{g} / \mathrm{L})$ (Lopez-Garcia et al. 2009). Another study from St. John's, Newfoundland measured boron concentrations of lactating mothers' milk for full-term infants to be 30 and $28 \mu \mathrm{g} / \mathrm{L}$ at 1 and 12 weeks postpartum, respectively. In lactating mothers' milk for preterm infants boron concentrations were found to be 37 and $27 \mu \mathrm{g} / \mathrm{L}$ at 1 and 12 weeks, respectively (Hunt et al. 2004). The concentration of boron was determined in archived milk collected (1980-1984) from lactating mothers of full-term, exclusively breastfed infants living in Houston, Texas. The results indicated that infants were supplied average concentrations of $42 \pm 6.5 \mu \mathrm{g} / \mathrm{L}$ at 1 month old and 35 $\pm 6.5 \mu \mathrm{g} / \mathrm{L}$ at 4 months old via breast milk (Hunt et al 2005). Hunt et al. (2004, 2005) indicated that boron concentrations over the first 4 months of lactating did not vary widely and were stable over time. The highest concentrations of boron detected in each study were $88 \mu \mathrm{g} / \mathrm{L}$ (Hunt et al. 2005) and $100 \mu \mathrm{g} / \mathrm{L}$ (Hunt et al. 2004).

Child health data needs relating to susceptibility are discussed in Section 3.12.2, Identification of Data Needs: Children's Susceptibility.

Exposure Registries. No exposure registries for boron were located. This substance is not currently one of the compounds for which a sub-registry has been established in the National Exposure Registry. The substance will be considered in the future when chemical selection is made for sub-registries to be established. The information that is amassed in the National Exposure Registry facilitates the epidemiological research needed to assess adverse health outcomes that may be related to exposure to this substance. 


\subsubsection{Ongoing Studies}

Ongoing studies pertaining to boron or boron compounds were identified in a search of the Federal Research in Progress database (FEDRIP 2009). A large comprehensive study on boron by C. Hunt at the United States Department of Agriculture in Grand Forks, North Dakota is collecting and analyzing dietary data and biochemical indices aimed at enhancing the quality of life through establishing mineral intakes, which support optimal bone health as well as general micronutrient roles in physiology and health.

D. Evans at Columbia University Health Sciences, New York, New York is sponsored by the Institute of Environmental Health Sciences to investigate the use of low-toxicity pesticides such as baits, gels, and boric acid. The USDA Water Management Research Unit in Parlier, California is attempting to develop strategies for sustainable agronomic systems for water reuse and bioremediation of soils impacted by the use of saline water containing high concentrations of boron and trace elements. B. Hoenisch at Colombia University is funded by the National Science Foundation to investigate validation of the boron/calcium proxy for surface seawater $\mathrm{pH}$ and application to measure anthropogenic ocean acidification (FEDRIP 2009). 


\section{ANALYTICAL METHODS}

The purpose of this chapter is to describe the analytical methods that are available for detecting, measuring, and/or monitoring boron, its metabolites, and other biomarkers of exposure and effect to boron. The intent is not to provide an exhaustive list of analytical methods. Rather, the intention is to identify well-established methods that are used as the standard methods of analysis. Many of the analytical methods used for environmental samples are the methods approved by federal agencies and organizations such as EPA and the National Institute for Occupational Safety and Health (NIOSH). Other methods presented in this chapter are those that are approved by groups such as the Association of Official Analytical Chemists (AOAC) and the American Public Health Association (APHA). Additionally, analytical methods are included that modify previously used methods to obtain lower detection limits and/or to improve accuracy and precision.

\subsection{BIOLOGICAL MATERIALS}

Methods for the determination of boron in biological materials are summarized in Table 7-1. Methods for the determination of boron in samples of toxicological interest have been summarized (Stokinger 1981; Van Ormer 1975). Total boron is usually determined after the material is digested or ashed. No techniques are available to quantitatively analyze for specific boron compounds in biological matrices (Culver et al. 1994a). The most common analytical procedure to analyze boron in biological materials involves digestion of the sample in hot acid or base, followed by analysis of the resulting solution by inductively coupled plasma-atomic emission spectrometry (ICP-AES) (Culver et al. 2001).

Goullé et al. (2005) assessed inductively coupled plasma-mass spectrometry (ICP-MS) for detecting metals and metalloids in whole blood, plasma, urine, and hair. ICP-MS is a fast, sensitive method that requires a small sample size. Analysis required $0.4 \mathrm{~mL}$ of blood, plasma, or urine and $25 \mathrm{mg}$ of hair. Usuda et al. (1998) noted that boron levels in urine can be influenced by dietary intake of boron and recommended that the intake of large amounts of boron-rich foods or drinks should be avoided if the boron status is being evaluated for possible environmental- or labor-related exposure. Xing et al (2008) suggested that creatinine-corrected, post-shift urine boron concentration can be used as a biomarker of human boron exposure in worker populations when monitored by ICP-MS.

Colorimetric analysis can also be used to determine boron concentrations in biological samples; however, colorimetric procedures are more time-consuming and require more laboratory care and technical skill 


\section{Table 7-1. Analytical Methods for Determining Boron in Biological Materials}

\begin{tabular}{|c|c|c|c|c|c|}
\hline Sample matrix & Preparation method & $\begin{array}{l}\text { Analytical } \\
\text { method }\end{array}$ & $\begin{array}{l}\text { Sample detection } \\
\text { limit }\end{array}$ & $\begin{array}{l}\text { Percent } \\
\text { recovery }\end{array}$ & Reference \\
\hline Urine & Dilution; direct analysis & ICP-AES & $6.24 \mu \mathrm{g} / \mathrm{L}$ & $\begin{array}{l}100.8- \\
104.2 \%\end{array}$ & $\begin{array}{l}\text { Usuda et al. } \\
1998\end{array}$ \\
\hline $\begin{array}{l}\text { Whole blood, } \\
\text { plasma, urine }\end{array}$ & $\begin{array}{l}\text { Dilution with purified } \\
\text { water, acid, buffer, and } \\
\text { butanol }\end{array}$ & ICP-MS & $\begin{array}{l}1.33 \mu \mathrm{g} / \mathrm{L} \text { (blood) } \\
1.26 \mu \mathrm{g} / \mathrm{L} \text { (plasma) } \\
0.25 \mu \mathrm{g} / \mathrm{L} \text { (urine) }\end{array}$ & No data & $\begin{array}{l}\text { Goullé et al. } \\
2005\end{array}$ \\
\hline Hair & $\begin{array}{l}\text { Mineralization after } \\
\text { decontamination with } \\
\text { water/acetone }\end{array}$ & ICP-MS & $0.14 \mathrm{ng} / \mathrm{mg}$ & No data & $\begin{array}{l}\text { Goullé et al. } \\
2005\end{array}$ \\
\hline Blood & $\begin{array}{l}\text { Ashed by oxygen in a } \\
\text { Parr bomb, dissolved }\end{array}$ & $\begin{array}{l}\text { Colorimetric } \\
\text { carmine } \\
\text { method }\end{array}$ & $<100 \mu \mathrm{g} / \mathrm{L}$ & $\begin{array}{l}84 \% \text { at } \\
5 \mu \mathrm{g} / \mathrm{mL}\end{array}$ & $\begin{array}{l}\text { Hill and } \\
\text { Smith } 1959\end{array}$ \\
\hline Serum (borate) & $\begin{array}{l}\text { Deproteinized, allowed to } \\
\text { react with reagent }\end{array}$ & $\begin{array}{l}\text { Colorimetric } \\
\text { carmine } \\
\text { method }\end{array}$ & $\begin{array}{l}\text { Greater than } \\
\text { endogenous levels, } \\
\text { which are } \\
<20 \mathrm{mg} / \mathrm{L}\end{array}$ & $92-104 \%$ & Baselt 1988 \\
\hline
\end{tabular}

ICP-AES = inductively coupled plasma-atomic emission spectroscopy; ICP-MS = inductively coupled plasma-mass spectrometry 
(Culver et al. 2001). Spectrophotometric methods suffer from interferences from other elements (e.g., Al, $\mathrm{Cu}, \mathrm{Fe}, \mathrm{Zn}$, and Mo), as well as $\mathrm{pH}$ (Sah and Brown 1997).

Neutron activation analysis (NAA) is another analytical technique used to determine boron in biological samples. In NAA, the sample is bombarded with neutrons, and the element of interest is made radioactive. The amount of the element present in the sample is then determined by measurement of the radioactivity or radioactive decay products. This process involves ${ }^{10} \mathrm{~B}$, which is a naturally occurring, stable isotope of boron that occurs with about $20 \%$ abundance. When ${ }^{10} \mathrm{~B}$ is bombarded with neutrons, it does not become radioactive, but results in a neutron-capture reaction, resulting in the emission of a $\alpha$-particle and $\gamma$-photon. NAA methods for boron determination are based on the measurement of one of more of the products ( $\alpha$-particle and $\gamma$-photon), using techniques such as neutron activation mass spectrometry (NA-MS) or prompt $\gamma$-ray spectroscopy. An advantage of NAA is that it is a nondestructive method. However, the requirements of a neutron source and the abundance of ${ }^{10} \mathrm{~B}$ restrict its use (Culver et al. 2001; Sah and Brown 1997).

\subsection{ENVIRONMENTAL SAMPLES}

Methods for the determination of boron in environmental samples are summarized in Table 7-2.

Boron is readily measured in multielement analyses of air, water, and solid waste samples by ICP-AES atomic emission spectroscopy, the method of choice for the determination of boron in modern practice. Although not multielement procedures, colorimetric cucumin and colorimetric carmine methods are still reliable methods for the determination of boron in water, air, and solid waste samples (APHA 1998b; 1998c; EPA 1983). These colorimetric procedures provide adequate methods when ICP instrumentation is not available. Alkali fusion or wet digestion with hydrofluoric acid or a mixture of hydrofluoric acid and other acids are used to digest soils and other geological and silica-rich materials (Sah and Brown 1997). A highly sensitive and selective method for determining boron at $\mathrm{ng} / \mathrm{dm}^{3}$ levels was developed by Takahashi et al. (2008) by derivatization reaction of boron with reversed-phase partition highperformance liquid chromatography (HPLC) separation and ultraviolet spectrophotometric detection. A detection limit as low as $22 \mathrm{ng} / \mathrm{dm}^{3}$ was achieved without any preconcentration. No significant interference was observed in the determination of $16 \mu \mathrm{mol} / \mathrm{dm}^{3}$ of boron with the addition of nine metal ions $\left(\mathrm{Al}^{3+}, \mathrm{CuI}^{2+}, \mathrm{Co}^{2+}, \mathrm{Fe}^{2+}, \mathrm{Fe}^{3+}, \mathrm{Ni}^{2+}, \mathrm{Mn}^{2+}, \mathrm{V}^{5+}\right.$, and $\mathrm{Zn}^{2+}$ ) at concentrations 100 times greater than that of boron (Takahashi et al. 2008). 
Table 7-2. Analytical Methods for Determining Boron in Environmental Samples

\begin{tabular}{|c|c|c|c|c|c|}
\hline $\begin{array}{l}\text { Sample } \\
\text { matrix }\end{array}$ & Preparation method & $\begin{array}{l}\text { Analytical } \\
\text { method }\end{array}$ & $\begin{array}{l}\text { Sample } \\
\text { detection limit }\end{array}$ & $\begin{array}{l}\text { Percent } \\
\text { recovery }\end{array}$ & Reference \\
\hline Air & $\begin{array}{l}\text { Collection on filter, hot } \\
\text { block/ } \mathrm{HCl} / \mathrm{HNO}_{3} \text { digestion } \\
\text { followed by dilution with water }\end{array}$ & $\begin{array}{l}\text { NIOSH } \\
\text { Method } 7303 \\
\text { ICP-AES }\end{array}$ & $\begin{array}{l}0.0094 \mu \mathrm{g} / \mathrm{L} \\
0.71 \mu \mathrm{g} / \mathrm{sample}\end{array}$ & No data & NIOSH 2003 \\
\hline $\begin{array}{l}\text { Air (boron } \\
\text { carbide) }\end{array}$ & $\begin{array}{l}\text { Collection on filter, ashed, } \\
\text { suspended in 2-propanol, } \\
\text { redeposited on silver } \\
\text { membrane filter }\end{array}$ & $\begin{array}{l}\text { NIOSH } \\
\text { Method } 7506 \\
\text { X-ray powder } \\
\text { diffraction }\end{array}$ & $\begin{array}{l}0.05 \mathrm{mg} / \\
\text { sample }\end{array}$ & No data & NIOSH 1994 \\
\hline $\begin{array}{l}\text { Air (boron } \\
\text { trifluoride) }\end{array}$ & $\begin{array}{l}\text { A known volume of air is } \\
\text { drawn through a solution of } \\
\text { ammonium fluoride; } \\
\text { fluoroborate ion is measured } \\
\text { using an ion specific electrode }\end{array}$ & $\begin{array}{l}\text { OSHA } \\
\text { Method } \\
\text { ID216SG } \\
\text { Fluoroborate } \\
\text { ion specific } \\
\text { electrode }\end{array}$ & $\begin{array}{l}10 \mu \mathrm{g} \text { or } \\
0.4 \mu \mathrm{g} / \mathrm{mL} \text { of } \\
\text { solution }\end{array}$ & No data & OSHA 1989b \\
\hline Water & Acidify, inject & $\begin{array}{l}\text { APHA } \\
\text { Method } 3120 \\
\text { ICP-AES }\end{array}$ & $5 \mu \mathrm{g} / \mathrm{L}$ & $\begin{array}{l}115.46 \%{ }^{a} \\
27 \% \operatorname{RDS}^{a}\end{array}$ & APHA 1998a \\
\hline Water & Direct analysis & $\begin{array}{l}\text { APHA } \\
\text { Method 4500- } \\
\text { B Colorimetric } \\
\text { curcumin }\end{array}$ & $0.2 \mu \mathrm{g}$ & $\begin{array}{l}22.8 \% \\
\text { RSD }\end{array}$ & APHA 1998b \\
\hline Water & Ash, dissolve in acid & $\begin{array}{l}\text { APHA } \\
\text { Method 4500- } \\
\text { B Colorimetric } \\
\text { carmine }\end{array}$ & $2 \mu \mathrm{g}$ & $\begin{array}{l}35.5 \% \\
\text { RSD }\end{array}$ & APHA 1998c \\
\hline Water & Direct analysis & $\begin{array}{l}\text { EPA Method } \\
212.3 \text { Colori- } \\
\text { metric } \\
\text { curcumin }^{b}\end{array}$ & $\begin{array}{l}0.1-1.0 \mathrm{mg} / \mathrm{L} \\
\text { (optimal range) }\end{array}$ & $\begin{array}{l}22.8 \% \\
\text { RSD }\end{array}$ & EPA 1983 \\
\hline Water & Filter, acidify & $\begin{array}{l}\text { EPA Method } \\
200.7 \text { ICP- } \\
\text { AES }\end{array}$ & $3 \mu \mathrm{g} / \mathrm{L}$ & $\begin{array}{l}115 \%^{\mathrm{a}} \\
27 \% \mathrm{RSD}^{\mathrm{a}}\end{array}$ & EPA 1994 \\
\hline $\begin{array}{l}\text { Water, } \\
\text { sediments, } \\
\text { solid wastes, } \\
\text { sludges }\end{array}$ & $\begin{array}{l}\text { Aqueous and solid matrices } \\
\text { require acid digestion prior to } \\
\text { analysis; pre-filtered, acidified } \\
\text { groundwater samples do not } \\
\text { need acid digestion }\end{array}$ & $\begin{array}{l}\text { EPA Method } \\
6010 C \text { ICP- } \\
\text { AES }\end{array}$ & $3.8 \mu \mathrm{g} / \mathrm{L}$ & No data & EPA 2000 \\
\hline Water & Direct analysis & $\begin{array}{l}\text { USGS-NWQL } \\
\text { Method I- } \\
1114 \text { DCP- } \\
\text { AES }\end{array}$ & $\begin{array}{l}\text { Applicable } \\
\text { range: } 10- \\
1,000 \mu \mathrm{g} / \mathrm{L}\end{array}$ & $5.8 \%$ RSD & USGS 1989 \\
\hline $\begin{array}{l}\text { Aquatic } \\
\text { biological } \\
\text { material }\end{array}$ & $\begin{array}{l}\text { Nitric acid digestion followed } \\
\text { by treatment with } 30 \% \\
\text { hydrogen peroxide }\end{array}$ & $\begin{array}{l}\text { USGS-NWQL } \\
\text { Method B- } \\
\text { 9001-95 ICP- } \\
\text { AES }\end{array}$ & & $95-96 \%$ & USGS 1996a \\
\hline
\end{tabular}




\section{Table 7-2. Analytical Methods for Determining Boron in Environmental Samples}

\begin{tabular}{|c|c|c|c|c|c|}
\hline $\begin{array}{l}\text { Sample } \\
\text { matrix }\end{array}$ & Preparation method & $\begin{array}{l}\text { Analytical } \\
\text { method }\end{array}$ & $\begin{array}{l}\text { Sample } \\
\text { detection limit }\end{array}$ & $\begin{array}{l}\text { Percent } \\
\text { recovery }\end{array}$ & Reference \\
\hline Water & $\begin{array}{l}\text { Acidify to a } \mathrm{pH}<2.0 \text { with nitric } \\
\text { acid }\end{array}$ & $\begin{array}{l}\text { USGS-NWQL } \\
\text { Method I- } \\
\text { 1472-95 ICP- } \\
\text { AES }\end{array}$ & $\begin{array}{l}\text { Method } \\
\text { reporting limit: } \\
4 \mu \mathrm{\mu g} / \mathrm{L}\end{array}$ & $91.6-109 \%$ & USGS 1996b \\
\hline Water & Filter, acidify & $\begin{array}{l}\text { USGS-NWQL } \\
\text { Method I- } \\
2477-92 \text { ICP- } \\
\text { AES }\end{array}$ & & $70-103 \%$ & USGS 1999 \\
\hline Water & Acidify, filter & $\begin{array}{l}\text { USGS-NWQL } \\
\text { Method I- } \\
4471-97 \text { ICP- } \\
\text { OES }\end{array}$ & $13 \mu \mathrm{g} / \mathrm{L}$ & $\begin{array}{l}98 \% \\
\text { (average) }\end{array}$ & USGS 1998 \\
\hline
\end{tabular}

${ }^{a}$ Percent recovery and relative standard deviation were reported by NEMI (2007).

${ }^{\mathrm{b}}$ Same method as APHA (1998b).

APHA = American Public Health Association; DCP = direct current plasma; EPA = U.S. Environmental Protection Agency; ICP-AES = inductively coupled plasma-atomic emission spectroscopy; ICP-OES = inductively coupled plasma-optical emission spectroscopy; $\mathrm{NIOSH}=$ National Institute for Occupational Safety and Health; NWQL = National Water Quality Laboratory; OSHA = Occupational Safety and Health Administration; RSD = relative standard deviation; USGS = U.S. Geological Survey 
Anthropogenic-derived boron concentrations can be effectively distinguished by the the measurement of the ${ }^{10} \mathrm{~B}$ to ${ }^{11} \mathrm{~B}$ isotopic ratio (Chetelat and Gaillardet 2005; Moore et al. 2008). The natural abundance of ${ }^{10} \mathrm{~B}$ and ${ }^{11} \mathrm{~B}$ is specific to location, making anthropogenic boron isotopic ratios distinctly different than groundwater (Davidson and Bassett 1993). This allows utilization of the boron isotopic ratio to determine potential contamination in groundwater (Vengosh et al. 1994), river water (Chetelat and Gaillardet 2005), marine water (Xiao et al. 2007), and effluent (Kloppmann et al. 2008).

\subsection{ADEQUACY OF THE DATABASE}

Section 104(i)(5) of CERCLA, as amended, directs the Administrator of ATSDR (in consultation with the Administrator of EPA and agencies and programs of the Public Health Service) to assess whether adequate information on the health effects of boron is available. Where adequate information is not available, ATSDR, in conjunction with NTP, is required to assure the initiation of a program of research designed to determine the health effects (and techniques for developing methods to determine such health effects) of boron.

The following categories of possible data needs have been identified by a joint team of scientists from ATSDR, NTP, and EPA. They are defined as substance-specific informational needs that if met would reduce the uncertainties of human health assessment. This definition should not be interpreted to mean that all data needs discussed in this section must be filled. In the future, the identified data needs will be evaluated and prioritized, and a substance-specific research agenda will be proposed.

\subsubsection{Identification of Data Needs}

Analytical methods are available and are adequately sensitive to detect boron in biological materials (e.g., blood and urine) and in environmental samples (e.g., water, sediments, and air). No data needs are identified at this time. 


\section{Methods for Determining Biomarkers of Exposure and Effect.}

Exposure. Boron can be determined sensitively and selectively by ICP-AES and ICP-MS in urine and blood (Goullé et al. 2005; Sah and Brown 1997; Usuda et al. 1998). Analytical methods with satisfactory sensitivity and precision are available to determine levels of boron in human tissues and body fluids.

Effect. Existing methods are sensitive enough to measure background levels for boron in the population and levels at which biological effects occur.

\section{Methods for Determining Parent Compounds and Degradation Products in Environmental}

Media. ICP-AES is a satisfactory multielement method available for the determination of boron in water, air, and solid waste samples (APHA 1998a; EPA 1994; 2000; NIOSH 2003; USGS 1989, 1996a, 1996b, 1999). Colorimetric procedures are as sensitive and precise, but are more labor intensive. Colorimetric procedures do provide adequate methods for those laboratories that do not have ICP instrumentation. An alternative method to the expensive and highly skill-dependent ICP-MS has been developed by boron derivatization and analysis by standard reverse-phase HPLC without sacrificing sensitivity (Takahashi et al. 2008).

Sampling methodologies for very low level elemental substances like boron continue to pose problems such as nonrepresentative samples, insufficient sample volumes, contamination, and labor-intensive, tedious extraction and purification procedures (Green and Le Pape 1987).

\subsubsection{Ongoing Studies}

No ongoing studies pertaining to analytical methods for boron were identified in a search of the Federal Research in Progress database (FEDRIP 2009). 
7. ANALYTICAL METHODS

This page is intentionally blank. 


\section{REGULATIONS, ADVISORIES, AND GUIDELINES}

MRLs are substance specific estimates, which are intended to serve as screening levels, are used by ATSDR health assessors and other responders to identify contaminants and potential health effects that may be of concern at hazardous waste sites.

ATSDR has derived an acute-duration inhalation MRL of $0.3 \mathrm{mg}$ boron $/ \mathrm{m}^{3}$ for boron. This MRL is based on a NOAEL of $0.8 \mathrm{mg}$ boron $/ \mathrm{m}^{3}$ for significantly increased nasal secretions in volunteers (Cain et al. 2004) and an uncertainty factor of $3\left(10^{0.5}\right.$ for human variability in the pharmacodynamic response to boron).

ATSDR has derived an acute-duration oral MRL of $0.2 \mathrm{mg}$ boron $/ \mathrm{kg} /$ day for boron. This MRL is based on a NOAEL of $22 \mathrm{mg}$ boron/kg/day associated with a LOAEL of $44 \mathrm{mg}$ boron/kg/day for increased incidence of external, visceral, and cardiovascular malformations and reduced body weight in the fetuses of rabbits administered boric acid via gavage on gestation days 6-19 (Price et al. 1996b) and an uncertainty factor of 100 (10 for interspecies extrapolation and 10 for human variability).

ATSDR has derived an intermediate-duration oral MRL of $0.2 \mathrm{mg}$ boron $/ \mathrm{kg} /$ day for boron. This MRL is based on a $\mathrm{BMDL}_{05}$ of $10.3 \mathrm{mg}$ boron/kg/day estimated from fetal body weight data from two studies in which pregnant rats were exposed to boron in the diet on gestation days 0-20 (Heindel et al. 1992; Price et al. 1996a) and chemical-specific uncertainty factor of 66 (3.3 for toxicokinetic extrapolation from animals to humans, 3.16 for toxicodynamic extrapolation from animals to humans, 2.0 for variability in human toxicokinetics, and 3.16 for variability in human toxicodynamics).

EPA has established an oral reference dose (RfD) of $0.2 \mathrm{mg} / \mathrm{kg} /$ day based on decreased fetal weight in a developmental study in Sprague-Dawley rats orally exposed to boric acid from gestation days 0 to 20 (IRIS 2007). EPA has not established an inhalation reference concentration (RfC) for boron and compounds.

Under the Federal Insecticide, Fungicide, and Rodenticide Act (FIFRA), boron oxide, boric acid, borax, and sodium tetraborate are exempt from tolerances for pesticide chemicals in food (EPA 2007b); they are also listed as inerts of unknown toxicity (List 3) in EPA's Categorized List of Inert Pesticide Ingredients (EPA 2004). 
8. REGULATIONS, ADVISORIES, AND GUIDELINES

The international and national regulations, advisories, and guidelines regarding boron in air, water, and other media are summarized in Table 8-1. 


\section{Table 8-1. Regulations, Advisories and Guidelines Applicable to Boron and Boron Compounds}

\begin{tabular}{|c|c|c|c|}
\hline Agency & Description & Information & Reference \\
\hline \multicolumn{4}{|c|}{ INTERNATIONAL } \\
\hline \multicolumn{4}{|l|}{ Guidelines: } \\
\hline IARC & Carcinogenicity classification & No data & IARC 2006 \\
\hline \multirow[t]{2}{*}{ WHO } & Air quality guidelines & No data & WHO 2000 \\
\hline & Drinking water quality guidelines (boron) & $0.5 \mathrm{mg} / \mathrm{L}^{\mathrm{a}}$ & WHO 2004 \\
\hline \multicolumn{4}{|l|}{ NATIONAL } \\
\hline \multicolumn{4}{|c|}{$\begin{array}{l}\text { Regulations and } \\
\text { Guidelines: }\end{array}$} \\
\hline \multicolumn{4}{|l|}{ a. Air } \\
\hline \multirow[t]{8}{*}{ ACGIH } & TLV (8-hour TWA) & & ACGIH 2006 \\
\hline & $\begin{array}{l}\text { Borate compounds, inorganic (borax, } \\
\text { boric acid, and sodium tetraborate) }\end{array}$ & $2 \mathrm{mg} / \mathrm{m}^{3}$ & \\
\hline & Boron oxide & $10 \mathrm{mg} / \mathrm{m}^{3}$ & \\
\hline & TLV (ceiling) & & \\
\hline & Boron tribromide & $10 \mathrm{mg} / \mathrm{m}^{3}$ & \\
\hline & Boron trifluoride & $3 \mathrm{mg} / \mathrm{m}^{3}$ & \\
\hline & STEL (15-minute TWA) & & \\
\hline & $\begin{array}{l}\text { Borate compounds, inorganic (borax, } \\
\text { boric acid, and sodium tetraborate) }\end{array}$ & $6 \mathrm{mg} / \mathrm{m}^{3}$ & \\
\hline \multirow[t]{4}{*}{ AlHA } & Boron trifluoride & & AlHA 1999 \\
\hline & ERPG-1 ${ }^{b}$ & $2 \mathrm{mg} / \mathrm{m}^{3}$ & \\
\hline & ERPG- $2^{b}$ & $30 \mathrm{mg} / \mathrm{m}^{3}$ & \\
\hline & ERPG- $3^{b}$ & $100 \mathrm{mg} / \mathrm{m}^{3}$ & \\
\hline \multirow[t]{15}{*}{ EPA } & AEGL-1 ${ }^{\mathrm{C}}$ (boron trifluoride) & & EPA 2007a \\
\hline & 10 minutes & $2.5 \mathrm{mg} / \mathrm{m}^{3}$ & \\
\hline & 30 minutes & $2.5 \mathrm{mg} / \mathrm{m}^{3}$ & \\
\hline & 60 minutes & $2.5 \mathrm{mg} / \mathrm{m}^{3}$ & \\
\hline & 4 hours & $2.5 \mathrm{mg} / \mathrm{m}^{3}$ & \\
\hline & 8 hours & $2.5 \mathrm{mg} / \mathrm{m}^{3}$ & \\
\hline & AEGL-2 $2^{c}$ (boron trifluoride) & & \\
\hline & 10 minutes & $47 \mathrm{mg} / \mathrm{m}^{3}$ & \\
\hline & 30 minutes & $47 \mathrm{mg} / \mathrm{m}^{3}$ & \\
\hline & 60 minutes & $37 \mathrm{mg} / \mathrm{m}^{3}$ & \\
\hline & 4 hours & $24 \mathrm{mg} / \mathrm{m}^{3}$ & \\
\hline & 8 hours & $12 \mathrm{mg} / \mathrm{m}^{3}$ & \\
\hline & AEGL- $3^{\mathrm{C}}$ (boron trifluoride) & & \\
\hline & 10 minutes & $140 \mathrm{mg} / \mathrm{m}^{3}$ & \\
\hline & 30 minutes & $140 \mathrm{mg} / \mathrm{m}^{3}$ & \\
\hline
\end{tabular}




\section{Table 8-1. Regulations, Advisories and Guidelines Applicable to Boron and Boron Compounds}

\begin{tabular}{|c|c|c|c|}
\hline Agency & Description & Information & Reference \\
\hline & 60 minutes & $110 \mathrm{mg} / \mathrm{m}^{3}$ & \\
\hline & 4 hours & $72 \mathrm{mg} / \mathrm{m}^{3}$ & \\
\hline & 8 hours & $36 \mathrm{mg} / \mathrm{m}^{3}$ & \\
\hline \multicolumn{4}{|c|}{ NATIONAL (cont.) } \\
\hline \multirow[t]{3}{*}{ EPA } & \multicolumn{2}{|l|}{$\begin{array}{l}\text { Regulated toxic substances and threshold } \\
\text { quantities for accidental release prevention } \\
\text { pursuant to Section } 112(r) \text { of the Clean Air } \\
\text { Act }\end{array}$} & $\begin{array}{l}\text { EPA 2008a } \\
40 \text { CFR } 68.130\end{array}$ \\
\hline & Boron trichloride & 5,000 pounds & \\
\hline & Boron trifluoride & 5,000 pounds & \\
\hline \multirow[t]{12}{*}{$\mathrm{NIOSH}$} & REL & & NIOSH 2005 \\
\hline & Borax (10-hour TWA) & $5 \mathrm{mg} / \mathrm{m}^{3}$ & \\
\hline & Boron oxide (10-hour TWA) & $10 \mathrm{mg} / \mathrm{m}^{3}$ & \\
\hline & Boron tribromide (ceiling) & $10 \mathrm{mg} / \mathrm{m}^{3}$ & \\
\hline & Boron trifluoride (ceiling) & $3 \mathrm{mg} / \mathrm{m}^{3}$ & \\
\hline & Sodium tetraborate (10-hour TWA) & $1 \mathrm{mg} / \mathrm{m}^{3}$ & \\
\hline & IDLH (30-minute exposure) & & \\
\hline & Borax & No data & \\
\hline & Boron oxide & $2,0000 \mathrm{mg} / \mathrm{m}^{3}$ & \\
\hline & Boron tribromide & No data & \\
\hline & Boron trifluoride & $70 \mathrm{mg} / \mathrm{m}^{3}$ & \\
\hline & Sodium tetraborate & No data & \\
\hline \multirow[t]{14}{*}{ OSHA } & PEL for general industry & & OSHA 2006c \\
\hline & Boron oxide, total dust (8-hour TWA) & $15 \mathrm{mg} / \mathrm{m}^{3}$ & 29 CFR 1910.1000 \\
\hline & Boron trifluoride (ceiling) & $3 \mathrm{mg} / \mathrm{m}^{3}$ & \\
\hline & PEL for shipyard industry & & OSHA 2006a \\
\hline & Boron oxide, total dust (8-hour TWA) & $15 \mathrm{mg} / \mathrm{m}^{3}$ & 29 CFR 1915.1000 \\
\hline & Boron tribromide (8-hour TWA) & $10 \mathrm{mg} / \mathrm{m}^{3}$ & \\
\hline & Boron trifluoride (ceiling) & $3 \mathrm{mg} / \mathrm{m}^{3}$ & \\
\hline & PEL for construction industry & & OSHA 2006b \\
\hline & Boron oxide, total dust (8-hour TWA) & $15 \mathrm{mg} / \mathrm{m}^{3}$ & 29 CFR 1926.55, \\
\hline & Boron tribromide (8-hour TWA) & $10 \mathrm{mg} / \mathrm{m}^{3}$ & \\
\hline & Boron trifluoride (ceiling) & $3 \mathrm{mg} / \mathrm{m}^{3}$ & \\
\hline & $\begin{array}{l}\text { Threshold quantity of highly hazardous } \\
\text { chemicals, toxics, and reactives }\end{array}$ & & $\begin{array}{l}\text { OSHA 2006d } \\
29 \text { CFR } 1910.119\end{array}$ \\
\hline & Boron trichloride & 2,500 pounds & \\
\hline & Boron trifluoride & 250 pounds & \\
\hline \multicolumn{4}{|l|}{ b. Water } \\
\hline EPA & $\begin{array}{l}\text { Drinking water contaminant candidate list } \\
\text { Boron }\end{array}$ & Yes & $\begin{array}{l}\text { EPA } 1998 \\
63 \text { FR } 10274\end{array}$ \\
\hline
\end{tabular}




\section{Table 8-1. Regulations, Advisories and Guidelines Applicable to Boron and Boron Compounds}

\begin{tabular}{|c|c|c|c|}
\hline Agency & Description & Information & Reference \\
\hline \multicolumn{4}{|c|}{ NATIONAL (cont.) } \\
\hline \multirow[t]{7}{*}{ EPA } & $\begin{array}{l}\text { Drinking water standards and health } \\
\text { advisories for boron }\end{array}$ & & EPA 2006 \\
\hline & 1-day health advisory for a $10-\mathrm{kg}$ child & $4 \mathrm{mg} / \mathrm{L}$ & \\
\hline & 10-day health advisory for a $10-\mathrm{kg}$ child & $0.9 \mathrm{mg} / \mathrm{L}$ & \\
\hline & DWEL & $7 \mathrm{mg} / \mathrm{L}$ & \\
\hline & Lifetime & $1 \mathrm{mg} / \mathrm{L}$ & \\
\hline & $10^{-4}$ Cancer risk & No data & \\
\hline & National primary drinking water standards & No data & EPA 2003 \\
\hline \multicolumn{4}{|l|}{ c. Food } \\
\hline \multirow[t]{4}{*}{ EPA } & $\begin{array}{l}\text { Inert pesticide ingredients in pesticide } \\
\text { products }\end{array}$ & & EPA 2004 \\
\hline & $\begin{array}{l}\text { Borax, boric oxide, boric acid, and } \\
\text { sodium tetraborate }\end{array}$ & List $3^{d}$ & \\
\hline & $\begin{array}{l}\text { Tolerances and exemptions from } \\
\text { tolerances for pesticide chemicals in food }\end{array}$ & & $\begin{array}{l}\text { EPA 2007c } \\
40 \text { CFR } 180.101\end{array}$ \\
\hline & $\begin{array}{l}\text { Borax, boron oxide, boric acid, and } \\
\text { sodium tetraborate }\end{array}$ & Yes & \\
\hline \multirow[t]{4}{*}{ FDA } & EAFUS & & FDA 2007 \\
\hline & $\begin{array}{l}\text { Borax, boric acid, and sodium } \\
\text { tetraborate }\end{array}$ & Yes $^{e}$ & \\
\hline & $\begin{array}{l}\text { Indirect food additives: adhesives and } \\
\text { components of coatings }\end{array}$ & & $\begin{array}{l}\text { FDA } 2006 \\
21 \text { CFR } 175.105\end{array}$ \\
\hline & Borax and boric acid & Yes & \\
\hline \multicolumn{4}{|l|}{ d. Other } \\
\hline \multirow[t]{2}{*}{ ACGIH } & Carcinogenicity classification & & ACGIH 2006 \\
\hline & $\begin{array}{l}\text { Borate compounds, inorganic (borax, } \\
\text { boric acid, and sodium tetraborate) }\end{array}$ & $A 4^{\dagger}$ & \\
\hline \multirow[t]{8}{*}{ EPA } & $\begin{array}{l}\text { Carcinogenicity classification (boron and } \\
\text { boron compounds) }\end{array}$ & No data ${ }^{g}$ & IRIS 2007 \\
\hline & RfC (boron and boron compounds) & Not recommended & \\
\hline & RfD (boron and boron compounds) & $0.2 \mathrm{mg} / \mathrm{kg} / \mathrm{day}$ & \\
\hline & $\begin{array}{l}\text { Superfund, emergency planning, and } \\
\text { community right-to-know; effective date of } \\
\text { toxic chemical release reporting }\end{array}$ & & $\begin{array}{l}\text { EPA 2007b } \\
40 \text { CFR } 372.65\end{array}$ \\
\hline & $\begin{array}{l}\text { Boron tribromide, boron trichloride, and } \\
\text { boron trifluoride }\end{array}$ & 01/01/95 & \\
\hline & $\begin{array}{l}\text { Extremely hazardous substances and their } \\
\text { threshold planning quantities }\end{array}$ & & $\begin{array}{l}\text { EPA } 2008 b \\
40 \text { CFR } 355\end{array}$ \\
\hline & Boron trichloride & 500 pounds & Appendix A \\
\hline & Boron trifluoride & 500 pounds & \\
\hline
\end{tabular}




\section{Table 8-1. Regulations, Advisories and Guidelines Applicable to Boron and Boron Compounds}

\begin{tabular}{llll}
\hline Agency & Description & Information & Reference \\
\hline NATIONAL (cont.) & Carcinogenicity classification & No data & NTP 2005 \\
\hline NTP & Car & \\
\hline
\end{tabular}

${ }^{a}$ Provisional guideline value because calculated guideline value is below the level that can be achieved through practical treatment methods, source protection, etc.

${ }^{b}$ ERPG-1 is the maximum airborne concentration below which nearly all individuals could be exposed for up to 1 hour without experiencing other than mild, transient health effects; ERPG-2 is the maximum airborne concentration below which nearly all individuals could be exposed for up to 1 hour without experiencing irreversible or other serious adverse effects; and ERPG-3 is the maximum airborne concentration below which nearly all individuals could be exposed for up to 1 hour without life-threatening health effects (AlHA 1999).

${ }^{\mathrm{c}} \mathrm{AEGL}-1$ is the airborne concentration of a substance above which it is predicted that the general population, including susceptible individuals, could experience notable discomfort, irritation, or certain asymptomatic nonsensory effects; AEGL-2 is the airborne concentration of a substance above which it is predicted that the general population, including susceptible individuals, could experience irreversible or other serious, long-lasting adverse health effects or an impaired ability to escape; and AEGL-3 is the airborne concentration of a substance above which it is predicted that the general population, including susceptible individuals, could experience life-threatening health effects or death (EPA 2007c).

List 3: inerts of unknown toxicity

${ }^{\mathrm{e}}$ The EAFUS list of substances contains ingredients added directly to food that FDA has either approved as food additives or listed or affirmed as GRAS.

${ }^{\mathrm{f}} \mathrm{A} 4$ : not classifiable as a human carcinogen

${ }^{9}$ Data are inadequate for an assessment of human carcinogenic potential.

ACGIH = American Conference of Governmental Industrial Hygienists; AEGL = Acute Exposure Guideline Levels; AlHA = American Industrial Hygiene Association; CFR = Code of Federal Regulations; DWEL $=$ drinking water equivalent level; EAFUS = Everything Added to Food in the United States; EPA = Environmental Protection Agency; ERPG = Emergency Response Planning Guidelines; FDA = Food and Drug Administration; FR = Federal Register; GRAS = Generally Recognized As Safe; IARC = International Agency for Research on Cancer; IDLH = immediately dangerous to life or health; IRIS = Integrated Risk Information System; NIOSH = National Institute for Occupational Safety and Health; NTP = National Toxicology Program; OSHA = Occupational Safety and Health Administration; $\mathrm{PEL}=$ permissible exposure limit; $\mathrm{REL}=$ recommended exposure limit; $\mathrm{RfC}=$ inhalation reference concentration; $\mathrm{RfD}=$ oral reference dose; STEL = short-term expsoure limit; TLV = threshold limit values; TWA = time-weighted average; $\mathrm{WHO}=$ World Health Organization 


\section{REFERENCES}

ACGIH. 2006. Boron. Threshold limit values for chemical substances and physical agents and biological exposure indices. Cincinnati, OH: American Conference of Governmental Industrial Hygienists, 14-15.

Adinolfi M. 1985. The development of the human blood-CSF-brain barrier. Dev Med Child Neurol 27(4):532-537.

Adlercreutz H. 1995. Phytoestrogens: Epidemiology and a possible role in cancer protection. Environ Health Perspect Suppl 103(7):103-112.

Agency for Toxic Substances and Disease Registry. 1989. Decision guide for identifying substancespecific data needs related to toxicological profiles; Notice. Agency for Toxic Substances and Disease Registry, Division of Toxicology. Fed Regist 54(174):37618-37634.

AIHA. 1999. Boron trifluoride. Emergency response planning guidelines (ERPG). Fairfax, VA: American Industrial Hygiene Association.

Akar D. 2007. Potential boron pollution in surface water, crop, and soil in the lower Buyuk Menderes Basin. Environ Eng Sci 24(9):1273-1279.

Alam F, Evans F, Mani G, et al. 2003. Boron halides. Kirk-Othmer encyclopedia of chemical technology. John Wiley \& Sons, Inc.

http://www.mrw.interscience.wiley.com/emrw/9780471238966/kirk/article/boroalam.a01/current/pdf. March 11, 2009.

Alimonti A, Fornarelli L, Coni E, et al. 1992. Assessment of trace element concentrations in human lungs of urban subjects. Microchem J 46(2):191-198.

Allen BC, Strong PL, Price CJ, et al. 1996. Benchmark dose analysis of developmental toxicity in rats exposed to boric acid. Fundam Appl Toxicol 32(2):194-204.

Allen GT, Blackford SH, Tabor VM, et al. 2001. Metals, boron, and selenium in Neosho madtom habitats in the Neosho River in Kansas, USA. Environ Monit Assess 66(1):1-21.

Altman PL, Dittmer DS. 1974. Biological handbooks: Biology data book. Vol. III. 2nd ed. Bethesda, MD: Federation of American Societies for Experimental Biology, 1987-2008, 2041.

Andersen ME, Krishnan K. 1994. Relating in vitro to in vivo exposures with physiologically based tissue dosimetry and tissue response models. In: Salem H, ed. Animal test alternatives: Refinement, reduction, replacement. New York: Marcel Dekker, Inc., 9-25.

Andersen ME, Clewell HJ, Gargas ML, et al. 1987. Physiologically based pharmacokinetics and the risk assessment process for methylene chloride. Toxicol Appl Pharmacol 87(2):185-205

APHA. 1998a. Method 3120 A. Introduction. Method 3120 B. Inductively coupled plasma (ICP) method. In: Clesceri LS, Greenberg AE, Eaton AD, eds. Standard methods for the examination of water and wastewater. 20th ed. Washington, DC: American Public Health Association. American Water Works Association. Water Environmental Federation, 3-37 to 3-43. 


\section{REFERENCES}

APHA. 1998b. Method 4500 BB. Curcumin method. In: Clesceri LS, Greenberg AE, Eaton AD, eds. Standard methods for the examination of water and wastewater. 20th ed. Washington, DC: American Public Health Association. American Water Works Association. Water Environmental Federation, 4-21 to 4-22.

APHA. 1998c. Method 4500 BC. Carmine method. In: Clesceri LS, Greenberg AE, Eaton AD, eds. Standard methods for the examination of water and wastewater. 20th ed. Washington, DC: American Public Health Association. American Water Works Association. Water Environmental Federation, 4-23.

Arslan M, Topaktas M, Rencuzogullari E. 2008. The effects of boric acid on sister chromatid exchanges and chromosome aberrations in cultured human lymphocytes. Cytotechnology 56(2):91-96.

Barber LB, Murphy SF, Verplanck PL, et al. 2006. Chemical loading into surface water along a hydrological, biogeochemical, and land use gradient: A holistic watershed approach. Environ Sci Technol 40(2):476-486.

Barnes DG, Dourson M. 1988. Reference dose (RfD): Description and use in health risk assessments. Regul Toxicol Pharmacol 8(4):471-486.

Barranco WT, Hudak PF, Eckhert CD. 2007. Evaluation of ecological and in vitro effects of boron on prostate cancer risk (United States). Cancer Causes Control 18:71-77.

Baselt RC. 1988. Borate. In: Biological monitoring methods for industrial chemicals. 2nd ed. Littleton, MA: PSG Publishing Company, Inc., 48-50.

Beckett WS, Oskvig R, Gaynor ME, et al. 2001. Association of reversible alopecia with occupational topical exposure to common borax-containing solutions (Comment in J Am Acad Dermatol 47(4):636637). J Am Acad Dermatol 44(4):599-602.

Benson WH, Birge WJ, Dorough HW. 1984. Absence of mutagenic activity of sodium borate (borax) and boric acid in the Salmonella preincubation test. Environ Toxicol Chem 3:209-214.

Berger GS, ed. 1994. Epidemiology of endometriosis. In: Endometriosis: Advanced management and surgical techniques. New York, NY: Springer-Verlag, 3-7.

Bertine KK, Goldberg ED. 1971. Fossil fuel combustion and the major sedimentary cycle. Science 173:233-235.

Beyer KH, Bergfeld WF, Berndt WO, et al. 1983. Final report on the safety assessment of sodium borate and boric acid. J Am Coll Toxicol 2(7):87-125.

Biggar JW, Fireman M. 1960. Boron adsorption and release by soils. Soil Sci Soc Proc 115-120.

Bingham FT, Page AL, Coleman NT, et al. 1971. Boron adsorption characteristics of selected amorphous soils from Mexico and Hawaii. Soil Sci Am Proc 35:546-550.

Butterwick L, de Oude N, Raymond K. 1989. Safety assessment of boron in aquatic and terrestrial environments. Ecotoxicol Environ Safety 17:339-371.

Cain WS, Jalowayski AA, Kleinman M, et al. 2004. Sensory and associated reactions to mineral dusts: Sodium borate, calcium oxide, and calcium sulfate. J Occup Environ Hyg 1:222-236. 


\section{REFERENCES}

Cain WS, Jalowayski AA, Schmidt R, et al. 2008. Chemesthetic responses to airborne mineral dusts: Boric acid compared to alkaline materials. Int Arch Occup Environ Health 81:337-345.

Chang BL, Robbins WA, Wei F, et al. 2006. Boron exposure in China: Exploring work and lifestyle factors to boron exposure. AAOHN J 54(10):435-443.

Chao TC, Maxwell SM, Lyen K, et al. 1991a. Mass poisoning in Perak, Malaysia or the Tale of the Nine Emperor Gods and rat tail noodles. J Forensic Sci Soc 31(2):283-288.

Chao TC, Maxwell SM, Wong SY. 1991b. An outbreak of aflatoxicosis and boric acid poisoning in Malaysia: A clinicopathological study. J Pathol 164(3):225-233.

Chapin RE, Ku WW, Kenney MA, et al. 1997. The effects of dietary boron on bone strength in rats. Fundam Appl Toxicol 35(2):205-215.

ChemIDplus. 2009. Boron and selected boron chemicals. ChemIDplus. Bethesda, MD: U.S. National Library of Medicine. http://sis.nlm.nih.gov/chemical.html. April 01, 2009.

Cherrington JW, Chernoff N. 2002. Periods of vertebral column sensitivity to boric acid treatment in CD-1 mice in utero. Reprod Toxicol 16(3):237-243.

Chetelat B, Gaillardet J. 2005. Boron isotopes in the Seine River, France: A probe of anthropogenic contamination. Environ Sci Technol 39(8):2486-2493.

Choi WW, Chen KY. 1979. Evaluation of boron removal by adsorption on solids. Environ Sci Technol 13(2):189-196.

Clewell HJ, Andersen ME. 1985. Risk assessment extrapolations and physiological modeling. Toxicol Ind Health 1(4):111-131.

Cotton FA, Wilkinson G, Murillo CA, et al., eds. 1999. Boron. In: Advanced inorganic chemistry. 6th ed. New York, NY: John Wiley \& Sons, Inc., 131-174.

Cozzolino D, Kwiatkowski MJ, Dambergs RG, et al. 2008. Analysis of elements in wine using near infrared spectroscopy and partial least squares regression. Talanta 74(4):711-716.

Cui Y, Winton MI, Zhang Z, et al. 2004. Dietary boron intake and prostate cancer risk. Oncol Rep 11:887-892.

Culver BD, Shen PT, Taylor TH, et al. 1994a. The relationship of blood- and urine-boron exposure in borax-workers and usefulness of urine-boron as an exposure marker. Environ Health Perspect 102:133137.

Culver BD, Smith RG, Brotherton RJ, et al. 1994b. Boron. In: Clayton GD, Clayton FE, eds. Patty's industrial hygiene and toxicology. Vol. II. Part F. 4th ed. New York, NY: John Wiley \& Sons, Inc., 4411-4448.

Culver BD, Strong PL, Murray JF. 2001. Boron. In: Bingham E, Cohrssen B, Powell CH, eds. Patty's toxicology. Vol. 3. 5th ed. New York, NY: John Wiley \& Sons, Inc., 519-582. 


\section{REFERENCES}

Davidson GR, Bassett RL. 1993. Application of boron isotopes for identifying contaminants such as fly ash leachate in groundwater. Environ Sci Technol 27(1):172-176.

Davies K. 1990. Human exposure pathways to selected organochlorines and PCBs in Toronto and Southern Ontario. Adv Environ Sci Technol 23:525-540.

Demerec M, Bertani G, Flint J. 1951. A survey of chemicals for mutagenic action on E. coli. American Naturalist 85:119-136.

Deverel SJ, Millard SP. 1988. Distribution and mobility of selenium and other trace elements in shallow groundwater of the western San Joaquin Valley, California. Environ Sci Technol 22:697-702.

Dieter MP. 1994. Toxicity and carcinogenicity studies of boric acid in male and female B6C3F1 mice. Environ Health Perspect Suppl 102(7):93-97.

Dixon RL, Lee IP, Sherins RJ. 1976. Methods to assess reproductive effects of environmental chemicals: Studies of cadmium and boron administered orally. Environ Health Perspect 13:59-67.

Dixon RL, Sherins RJ, Lee IP. 1979. Assessment of environmental factors affecting male fertility. Environ Health Perspect 30:53-68.

Dourson M, Maier A, Meek B, et al. 1998. Boron tolerable intake: Re-evaluation of toxicokinetics for data-derived uncertainty factors. Biol Trace Elem Res 66:453-463.

Draize JH, Kelley EA. 1959. The urinary excretion of boric acid preparations following oral administration and topical applications to intact and damaged skin of rabbits. Toxicol Appl Pharmacol 1:267-276.

Dunlop W. 1981. Serial changes in renal haemodynamics during normal human pregnancy. Br J Obstet Gynaecol 88(1):1-9.

Eckel W, Langley WD. 1988. A background-based ranking technique for assessment of elemental enrichment in soils at hazardous waste sites. In: Superfund '88: Proceedings of the 9th National Conference, November 28-30, 1988, Washington, D.C. Silver Spring, MD: The Hazardous Materials Control Research Institute, 282-286.

Ellenhorn MJ, Schonwald S, Ordog G, et al., eds. 1997. Ellenhorn's medical toxicology: Diagnosis and treatment of human poisoning. 2nd ed. Baltimore, MA: Williams \& Wilkins, 106t, 162t, 1098-1100.

Ellenhorn MJ, Barceloux DG. 1988. Medical toxicology: Diagnosis and treatment of human poisoning. New York, NY: Elsevier, 921-924.

EPA. 1983. Method 212.3. Boron (colorimetric, curcumin). In: EPA methods and guidance for analysis of water. U.S. Environmental Protection Agency.

http://web1.er.usgs.gov/nemi/method_pdf/5279.pdf. March 22, 2007.

EPA. 1986a. Broad scan analysis of the FY82 national human adipose tissue survey specimens: Vol. I. Executive summary. Washington, DC: U.S. Environmental Protection Agency, Office of Toxic Substances. EPA560586035. 
EPA. 1986b. Quality criteria for water. Washington, DC: U.S. Environmental Protection Agency, Office of Water Planning and Standards. EPA440586001.

EPA. 1987. Toxic air pollutant/source crosswalk: A screening tool for locating possible sources emitting toxic air pollutants. Research Triangle Park, NC: U.S. Environmental Protection Agency, Office of Air Quality Planning and Standards. EPA450487023a. PB88161146.

EPA. 1990. Interim methods for development of inhalation reference concentrations. Washington, DC: U.S. Environmental Protection Agency, Office of Health and Environmental Assessment, Office of Research and Development, Environmental Criteria and Assessment Office. EPA600890066A.

EPA. 1993. Reregistration Eligibility Decision (RED). Boric acid and its sodium salts. Washington, DC: U.S. Environmental Protection Agency. EPA738F93006.

EPA. 1994. Method 200.7. Determination of metals and trace elements in water and wastes by inductively coupled plasma-atomic emission spectrometry. In: EPA methods and guidance for analysis of water. U.S. Environmental Protection Agency. http://web1.er.usgs.gov/nemi/method_pdf/4690.pdf. March 22, 2007.

EPA. 1997. Special report on environmental endocrine disruption: An effects assessment and analysis. Washington, DC: U.S. Environmental Protection Agency, Risk Assessment Forum. EPA630R96012.

EPA. 1998. The drinking water contaminant candidate list. Washington, DC: U.S. Environmental Protection Agency. Fed Regist 63:10274. http://www.gpoaccess.gov/fr/index.html. April 11, 2007.

EPA. 2000. Method 6010C. Inductively coupled plasma-atomic emission spectrometry. In: EPA methods and guidance for analysis of water. U.S. Environmental Protection Agency. http://web1.er.usgs.gov/nemi/method_pdf/4712.pdf. March 21, 2007.

EPA. 2003. National primary drinking water regulations. Washington, DC: U.S. Environmental Protection Agency, Office of Ground Water and Drinking Water. EPA816F03016. http://www.epa.gov/safewater/mcl.html. March 07, 2006.

EPA. 2004. Inert pesticide ingredients in pesticide products. Washington, DC: U.S. Environmental Protection Agency. http://www.epa.gov/opprd001/inerts/lists.html. April 11, 2007.

EPA. 2005. Toxic chemical release inventory reporting forms and instructions: Revised 2004 version. Section 313 of the Emergency Planning and Community Right-to-Know Act (Title III of the Superfund Amendments and Reauthorization Act of 1986). U.S. Environmental Protection Agency. Office of Environmental Information. EPA260B05001.

EPA. 2006. Drinking water standards and health advisories. Washington, DC: Office of Water, U.S. Environmental Protection Agency. EPA822R04005. http://epa.gov/waterscience/criteria/drinking/. April 11, 2007.

EPA. 2007a. Boron trifluoride. Acute exposure guideline levels (AEGLs). Washington, DC: Office of Pollution Prevention and Toxics, U.S. Environmental Protection Agency. http://www.epa.gov/oppt/aegl/pubs/compiled.pdf. April 11, 2007. 


\section{REFERENCES}

EPA. 2007b. Tolerances and exemptions from tolerances for pesticide chemicals in food. Washington, DC: U.S. Environmental Protection Agency. Code of Federal Regulations. 40 CFR 180.101. http://www.epa.gov/epacfr40/chapt-I.info/chi-toc.htm. March 12, 2009.

EPA. 2008a. Regulated toxic substances and threshold quantities for accidental release prevention. Washington, DC: U.S. Environmental Protection Agency. Code of Federal Regulations. 40 CFR 68.130. http://edocket.access.gpo.gov/cfr_2008/julqtr/pdf/40cfr68.130.pdf. March 31, 2009..

EPA. 2008b. Superfund, emergency planning, and community right-to-know programs. Extremely hazardous substances and their threshold planning quantities. Washington, DC: U.S. Environmental Protection Agency. Code of Federal Regulations. 40 CFR 355, Appendix A. http://edocket.access.gpo.gov/cfr_2008/julqtr/pdf/40cfr355AppA.pdf. March 31, 2009.

Fail PA, Chapin RE, Price CJ, et al. 1998. General, reproductive, developmental, and endocrine toxicity of boronated compounds. Reprod Toxicol 12(1):1-18.

Fail PA, George JD, Seely JC, et al. 1991. Reproductive toxicity of boric acid in Swiss (CD-1) mice: Assessment using the continuous breeding protocol. Fundam Appl Toxicol 17(2):225-239.

Farr LE, Konikowski T. 1963. The renal clearance of sodium pentaborate in mice and men. Clin Chem 9(6):717-726.

FDA. 2006. Indirect food additives: adhesives and components of coatings. Washington, DC: U.S. Food and Drug Administration. Code of Federal Regulations. 21 CFR 175.105. http://www.accessdata.fda.gov/scripts/cdrh/cfdocs/cfcfr/CFRSearch.cfm. April 11, 2007.

FDA. 2007. Everything added to food in the United States (EAFUS). Washington, DC: U.S. Food and Drug Administration. http://vm.cfsan.fda.gov/ dms/eafus.html. April 11, 2007.

FEDRIP. 2009. Boron and selected boron compounds. Federal Research in Progress database. Springfield, VA: National Technical Information Service.

Ferrando AA, Green NR. 1993. The effect of boron supplementation on lean body mass, plasma testosterone levels, and strength in male bodybuilders. Int J Sport Nutr 3(2):140-149.

Fisher RS, Freimuth HC. 1958. Blood boron levels in human infants. J Invest Dermatol 30:85-86.

FNB/IOM. 2001. Dietary reference intakes for vitamin A, vitamin K, arsenic, boron, chromium, copper, iodine, iron, manganese, molybdenum, nickel, silicon, vanadium, and zinc (2000). A Report of the Panel on Micronutrients, subcommittees on upper reference levels of nutrients and of interpretation and uses of dietary reference intakes, and the standing committee on the scientific evaluation of dietary reference intakes. Food and Nutrition Board. Institute of Medicine. Washington, DC: National Academy Press, 510-520. http://books.nap.edu/openbook.php?record_id=10026\&page=R2. August 06, 2007.

Fomon SJ. 1966. Body composition of the infant: Part I: The male reference infant. In: Falkner F, ed. Human development. Philadelphia, PA: WB Saunders, 239-246.

Fomon SJ, Haschke F, Ziegler EE, et al. 1982. Body composition of reference children from birth to age 10 years. Am J Clin Nutr 35(Suppl 5):1169-1175. 


\section{REFERENCES}

Fox KK, Cassani G, Facchi A, et al. 2002. Measured variation in boron loads reaching European sewage treatment works. Chemosphere 47(5):499-505.

Fukuda R, Hirode M, Mori I, et al. 2000. Collaborative work to evaluate toxicity on male reproductive organs by repeated dose studies in rats. 24). Testicular toxicity of boric acid after 2- and 4-week administration periods. J Toxicol Sci 25:233-239.

Gallardo-Williams MT, Chapin RE, King PE, et al. 2004. Boron supplementation inhibits the growth and local expression of IGF-1 in human prostate adenocarcinoma (LNCaP) tumors in nude mice. Toxicol Pathol 32:73-78.

Garabrant DH, Bernstein L, Peters JM, et al. 1984. Respiratory and eye irritation from boron oxide and boric acid dusts. J Occup Med 26(8):584-586.

Garabrant DH, Bernstein L, Peters JM, et al. 1985. Respiratory effects of borax dust. Br J Ind Med 42:831-837.

Giwercman A, Carlsen E, Keiding N, et al. 1993. Evidence for increasing incidence of abnormalities of the human testis: A review. Environ Health Perspect Suppl 101(2):65-71.

Goldfrank LR, Flomenbaum NE, Lewin NA, et al. 1990. Goldfrank's toxicologic emergencies. 4th ed. Norwalk, CT: Appleton \& Lange, 664-665.

Goldfrank LR, Flomenbaum NE, Lewin NA, et al., eds. 2002. Goldfrank's toxicologic emergencies. 7th ed. New York, NY: McGraw-Hill, 1282, 1289-1290, 1134.

Gonzales M, Shah V, Bobelu A, et al. 2004. Concentrations of surface-dust metals in Native American jewelry-making homes in Zuni Pueblo, New Mexico. Arch Environ Health 59(5):245-249.

Gordon AS, Prichard JS, Freedman MH. 1973. Seizure disorders and anemia associated with chronic borax intoxication. Can Med Assoc J 108:719-721, 724.

Goullé JP, Mahieu L, Castermant J, et al. 2005. Metal and metalloid multi-elementary ICP-MS validation in whole blood, plasma, urine and hair. Forensic Sci Int 153(1):39-44.

Graedel TE. 1978. Inorganic elements, hydrides, oxides and carbonates. In: Chemical compounds in the atmosphere. New York, NY: Academic Press, 35-49.

Green DR, Le Pape D. 1987. Stability of hydrocarbon samples on solid-phase extraction columns. Anal Chem 59:699-703.

Griffin RA, Burau RG. 1974. Kinetic and equilibrium studies of boron desorption from soil. Soil Sci Soc Am Proc 38:892-897.

Guzelian PS, Henry CJ, Olin SS, eds. 1992. Similarities and differences between children and adults: Implications for risk assessment. Washington, DC: International Life Sciences Institute Press.

Hall LW, Killen WD, Anderson RD, et al. 2004. Analysis of historical salinity and boron surface water monitoring data from the San Joaquin river watershed: 1985-2002. Environ Monit Assess 95(1-3):125151. 
Harris MA, Chapin RE, Lockhart AC, et al. 1992. Assessment of short-term reproductive and developmental toxicity screen. Fundam Appl Toxicol 19(2):186-196.

Haworth S, Lawlor T, Mortelmans K, et al. 1983. Salmonella mutagenicity test results for 250 chemicals. Environ Mutagen 5(Suppl 1):3-142.

HazDat. 2007. Boron. HazDat Database: ATSDR's Hazardous Substance Release and Health Effects Database. Atlanta, GA: Agency for Toxic Substances and Disease Registry. http://www.atsdr.cdc.gov/hazdat.html. April 23, 2007.

Heindel JJ, Price CJ, Field EA, et al. 1992. Developmental toxicity of boric acid in mice and rats. Fundam Appl Toxicol 18(2):266-277.

Heindel JJ, Price CJ, Schwetz BA. 1994. The developmental toxicity of boric acid in mice, rats, and rabbits. Environ Health Perspect Suppl 102(7):107-112.

Hill WH, Smith RC. 1959. Analysis of blood for boron. Am Ind Hyg Assoc J 20:131-133.

Hoel DG, Davis DL, Miller AB, et al. 1992. Trends in cancer mortality in 15 industrialized countries, 1969-1986. J Natl Cancer Inst 84(5):313-320.

Hollis JF, Keren R, Gal M. 1988. Boron release and sorption by fly ash as affected by pH and particle size. J Environ Qual 17:181-184.

Howe PD. 1998. A review of boron effects in the environment. Biol Trace Elem Res 66(1-3):153-166.

HSDB. 2009. Boron and selected boron chemicals. Hazardous Substances Data Bank. National Library of Medicine. http://toxnet.nlm.nih.gov/cgi-bin/sis/htmlgen?HSDB. April 01, 2009.

Hu X, Wegman DH, Eisen EA, et al. 1992. Dose related acute irritant symptom responses to occupational exposure to sodium borate dusts. Br J Ind Med 49(10):706-713.

Hubbard SA. 1998. Comparative toxicology of borates. Biol Trace Elem Res 66:343-357.

Hunt CD, Meacham SL. 2001. Aluminum, boron, calcium, copper, iron, magnesium, manganese, molybdenum, phosphorus, potassium, sodium, and zinc: Concentrations in common Western food and estimated daily intakes by infants, toddlers, and male and female adolescents, adults, and seniors in the United States. J Am Diet Assoc 101:1058-1060.

Hunt CD, Butte NF, Johnson, LK. 2005. Boron concentrations in milk from mothers of exclusively breast-fed healthy full-term infants are stable during the first four months of lactation. J Nutr 135(10):2383-2386.

Hunt CD, Friel JK, Johnson LK. 2004. Boron concentrations in milk from mothers of full-term and premature infants. Am J Clin Nutr 80(5):1327-1333.

Hunt CD, Shuler TR, Mullen LM. 1991. Concentration of boron and other elements in human foods and personal-care products. J Am Diet Assoc 91(5):558-568. 


\section{REFERENCES}

IARC. 2006. Agents reviewed by the IARC monographs. Volumes 1-96. Lyon, France: International Agency for Research on Cancer. http://monographs.iarc.fr/ENG/Classification/index.php. April 11, 2007.

Imbus HR, Cholak J, Miller LH, et al. 1963. Boron, cadmium, chromium, and nickel in blood and urine. Arch Environ Health 6:286-295.

IRIS. 2007. Boron and boron compounds. Washington, DC: Integrated Risk Information System. U.S. Environmental Protection Agency. http://www.epa.gov/iris/subst/index.html. April 11, 2007.

Ishii Y, Fujizuka N, Takahashi T, et al. 1993. A fatal case of acute boron poisoning. J Toxicol Clin Toxicol 31(2):345-352.

Jackson RE, Reddy KJ. 2007. Trace element chemistry of coal bed natural gas produced water in the Powder River Basin, Wyoming. Environ Sci Technol 41(17):5953-5959.

Jansen JA, Anderson J, Schou JS. 1984b. Boric acid single dose pharmacokinetics after intravenous administration to man. Arch Toxicol 55:64-67.

Jansen JA, Schou JS, Aggerback B. 1984a. Gastro-intestinal absorption and in vitro release of boric acid from water-emulsifying ointments. Food Chem Toxicol 22:49-53.

Jansen LH. 2003. Boron, elemental. In: Kirk-Othmer encyclopedia of chemical technology. John Wiley \& Sons, Inc.

http://www.mrw.interscience.wiley.com/emrw/9780471238966/kirk/article/borojans.a01/current/pdf. March 11, 2009.

Jensen AA. 2009. Risk assessment of boron in glass wool insulation. Environ Sci Pollut Res 16:73-78.

Johanson CE. 1980. Permeability and vascularity of the developing brain: Cerebellum vs cerebral cortex. Brain Res 190(1):3-16.

Keren R, Mezuman U. 1981. Boron adsorption by clay minerals using a phenomenological equation. Clays Clay Miner 29:198-204.

Keren R, Gast RG, Bar-Yosef B. 1981. pH-dependent boron adsorption by Na-montmorillonite. Soil Sci Soc Am J 45:45-48.

Kloppmann W, Van Houtte E, Picot G, et al. 2008. Monitoring reverse osmosis treated wastewater recharge into a coastal aquifer by environmental isotopes (B, Li, O, H). Environ Sci Technol 14(23):8759-8765.

Koc C. 2007. Effects on environment and agriculture of geothermal wastewater and boron pollution in Great Menderes Basin. Environ Monit Assess 125(1-3):377-388.

Komori M, Nishio K, Kitada M, et al. 1990. Fetus-specific expression of a form of cytochrome P-450 in human livers. Biochemistry 29(18):4430-4433.

Korkmaz M, Uzgoren E, Bakirdere S, et al. 2007. Effects of dietary boron on cervical cytopathology and on micronucleus frequency in exfoliated buccal cells. Environ Toxicol 22(1):17-25. 
Krishnan K, Andersen ME. 1994. Physiologically based pharmacokinetic modeling in toxicology. In: Hayes AW, ed. Principles and methods of toxicology. 3rd ed. New York, NY: Raven Press, Ltd., 149188.

Krishnan K, Anderson ME, Clewell HJ, et al. 1994. Physiologically based pharmacokinetic modeling of chemical mixtures. In: Yang RSH, eds. Toxicology of chemical mixtures. New York, NY: Academic Press, 399-437.

Krutzen E, Olofsson P, Back SE, et al. 1992. Glomerular filtration rate in pregnancy: a study in normal subjects and in patients with hypertension, preeclampsia and diabetes. Scand J Clin Lab Invest 52(5):387-392.

Krystofiak SP, Schaper MM. 1996. Prediction of an occupational exposure limit for a mixture on the basis of its components: Application to metalworking fluids. Am Ind Hyg Assoc J 57(3):239-244.

Ku WW, Chapin RE, Moseman RF, et al. 1991. Tissue disposition of boron in male Fischer rats. Toxicol Appl Pharmacol 111:145-151.

Ku WW, Chapin RE, Wine RN, et al. 1993a. Testicular toxicity of boric acid (BA): Relationship of dose to lesion development and recovery in the F344 rat. Reprod Toxicol 7(4):305-319.

Ku WW, Shih LM, Chapin RE. 1993b. The effects of boric acid (BA) on testicular cells in culture. Reprod Toxicol 7:321-331.

Kudo S, Tanase H, Yamasaki M, et al. 2000. Collaborative work to evaluate toxicity on male reproductive organs by repeated dose studies in rats. A comparative 2- and 4-week repeated oral dose testicular toxicity study of boric acid in rats. J Toxicol Sci 25:223-232.

Landolph JR. 1985. Cytotoxicity and negligible genotoxicity of borax and borax ores to cultured mammalian cells. Am J Ind Med 7:31-43.

Lang FJ, Bingham FT, Hendrix FF, et al. 1986. Boron deposition on soil and native vegetation from geothermal emissions. J Environ Qual 15(3):260-265.

Lee IP, Sherins RJ, Dixon RL. 1978. Evidence for induction of germinal aplasia in male rats by environmental exposure to boron. Toxicol Appl Pharmacol 45:577-590.

Leeder JS, Kearns GL. 1997. Pharmcogenetics in pediatrics: Implications for practice. Pediatr Clin North Am 44(1):55-77.

Leung H. 1993. Physiologically-based pharmacokinetic modeling. In: Ballentyne B, Marrs T, Turner P, eds. General and applied toxicology. Vol. 1. New York, NY: Stockton Press, 153-164.

Lide DR. 2008. CRC handbook of chemistry and physics. 2007-2008. 86th ed. Boca Raton, FL: CRC Press. Taylor \& Francis Group, 4-6 to 4-7.

Linden CH, Hall AH, Kulig KW, et al. 1986. Acute ingestions of boric acid. Clin Toxicol 24:269-279.

Litovitz TL, Klein-Schwartz W, Oderda GM, et al. 1988. Clinical manifestations of toxicity in a series of 784 boric acid ingestions. Am J Emerg Med 6:209-213. 


\section{REFERENCES}

Liu P, Hu W, Wei FS, et al. 2006a. Effect of boron on human semen quality. Chin J Public Health 22:940-942.

Liu P, Hu W, Gooping W, et al. 2006b. Effect of occupational exposure to boron on the sperm quality of males. Chin J Ind Occup Dis 24:167-169.

Livingston AL. 1978. Forage plant estrogens. J Toxicol Environ Health 4(2-3):301-324.

Locatelli C, Minoia C, Tonini M, et al. 1987. Human toxicology of boron with special reference to boric acid poisoning. G Ital Med Lav 9:141-146.

Lopez-Garcia I, Vinas P, Romero-Romero R, et al. 2009. Preconcentration and determination of boron in milk, infant formula, and honey samples by solid phase extraction-electrothermal atomic absorption spectrometry. Spectrochim Acta Part B 64B(2):179-183.

Lowe TP, Day DD. 2002. Metal concentrations in zebra mussels and sediments from embayments and riverine environments of eastern Lake Erie, southern Lake Ontario, and the Niagara River. Arch Environ Contam Toxicol 43(3):301-308.

Matthijs E, Holt MS, Kiewiet A, et al. 1999. Environmental monitoring for linear alkylbenzene sulfonate, alcohol ethoxylate, alcohol ethoxy sulfate, alcohol sulfate, and soap. Environ Toxicol Chem 18(11):2634-2644.

Maurer JK, Molai A, Parker RD, et al. 2001. Pathology of ocular irritation with bleaching agents in the rabbit low-volume eye test. Toxicol Pathol 29(3):308-319.

Mayr U, Butsch A, Schneider S. 1992. Validation of two in vitro test systems for estrogenic activities with zearalenone, phytoestrogens and cereal extracts. Toxicology 74(2-3):135-149.

McCabe LJ, Symons JM, Lee RD, et al. 1970. Survey of community water supply systems. J Am Water Work Assoc 62:670-687.

Meacham S, Hunt CD. 1994. Boron content of commercial baby foods and beverages typically consumed by infants. FASEB J 8(4-5):A430.

Mezuman U, Keren R. 1981. Boron adsorption by soils using a phenomenological equation. Soil Sci Soc Am J 45:722-726.

Minoia C, Sabbioni E, Ronchi A, et al. 1994. Trace element reference values in tissues from inhabitants of the European Community. IV. Influence of dietary factors. Sci Total Environ 141:181-195.

Moore SJ, Bassett RL, Liu B, et al. 2008. Geochemical tracers to evaluate hydrogeologic controls on river salination. Ground Water 46(3):489-501.

Morselli PL, Franco-Morselli R, Bossi L. 1980. Clinical pharmacokinetics in newborns and infants: Age-related differences and therapeutic implications. Clin Pharmacokin 5(6):485-527.

Moseman RF. 1994. Chemical disposition of boron in animals and humans. Environ Health Perspect 102:113-117. 


\section{REFERENCES}

Mumma RO, Raupach DC, Waldman JP, et al. 1984. National survey of elements and other constituents in municipal sewage sludges. Arch Environ Contam Toxicol 13:75-83.

Naderi AS, Palmer BF. 2006. Successful treatment of a rare case of boric acid overdose with hemodialysis. Am J Kidney Dis 48(6):95-97.

NAS/NRC. 1989. Report of the oversight committee. In: Biologic markers in reproductive toxicology. Washington, DC: National Academy of Sciences, National Research Council, National Academy Press, 15-35.

NEMI. 2007. Boron and selected boron compounds. National Environmental Methods Index. U.S. Environmental Protection Agency. U.S. Geological Survey. http://www.nemi.gov. August 06, 2007.

Nielsen FH, Hunt CD, Muller LM, et al. 1987. Effect of dietary boron on mineral, estrogen, and testosterone metabolism in postmenopausal women. FASEB Monogr 1:394-397.

NIH. 2004. Boric acid, sodium borate decahydrate (borax), and sodium tetraborate anhydrous. Household products database. National Institutes of Health. U.S. National Library of Medicine. http://hpd.nlm.nih.gov/ingredients.htm. March 13, 2009.

NIOSH. 1994. Boron carbide. Method 7506. NIOSH manual of analytical methods (NMAM). 4th ed. National Institute for Occupational Safety and Health. http://www.cdc.gov/niosh/nmam/pdfs/7506.pdf. March 14, 2007.

NIOSH. 2003. Method 7303. Elements by ICP. (Hot block/HCl/HNO3 digestion). In: NIOSH manual of analytical manual. (NMAM). National Institute for Occupational Safety and Health. http://www.cdc.gov.niosh/nmam/pdfs/7303.pdf. March 14, 2007.

NIOSH. 2005. NIOSH pocket guide to chemical hazards. Boron oxide. Atlanta, GA: National Institute for Occupational Safety and Health, Centers for Disease Control and Prevention.

http://www.cdc.gov/niosh/npg/. April 11, 2007.

NIOSH. 2007. Boron compounds. International chemical safety cards (ICSCs): U.S. National Version. National Institute for Occupational Safety and Health. http://www.cdc.gov/niosh/ipcs/nicstart.html. March 14, 2007.

NIRS. 1987. National inorganics and radionuclides survey. Distribution tables. Cincinnati, OH: U.S. Environmental Protection Agency, Office of Drinking Water.

NOES. 1989. National Occupational Exposure Survey. Cincinnati, OH: National Institute of Occupational Safety and Health. October 18, 1989.

NOHS. 1989. National Occupational Hazard Survey. National Institute of Occupational Safety and Health, Cincinnati, OH. October 18, 1989.

Nolte J. 1988. Pollution source analysis of river water and sewage sludge. Environ Technol Lett 9:857868.

Nomiyama T, Omae K, Uemura T, et al. 1995. No-observed-effect level of diborane on the respiratory organs of male mice in acute and subacute inhalation experiments. Sangyo Eiseigaku Zasshi 37(3):157160. 
Nriagu JO. 1979. Copper in the atmosphere and precipitation. In: Nriagu JO, ed. Copper in the environment. Part I: Ecological cycling. New York, NY: John Wiley and Sons, Inc., 43-67.

NRC. 1993. Pesticides in the diets of infants and children. Washington, DC: National Research Council. National Academy Press. PB93216091.

NTP. 1987. National Toxicology Program--technical report series no. TR324 on the toxicology and carcinogenesis studies of boric acid (CAS No. 10043-35-3) in B6C3F1 mice (feed studies). Research Triangle Park, NC: U.S. Department of Health and Human Services, Public Health Service, National Institutes of Health. NIH Publication No. 88-2580.

NTP. 1990. National Toxicology Program. Final report on the reproductive toxicity of boric acid in CD1-Swiss mice. National Institute of Environmental Health Sciences. NTP Report No. 90-105.

NTP. 1991. Final report on the developmental toxicity of boric acid (CAS No. 10043-35-3) in New Zealand white rabbits. Research Triangle Park, NC: U.S. Department of Health and Human Services, Public Health Service, National Toxicology Program. PB92129550.

NTP. 2005. Report on carcinogens. 11th ed. Research Triangle Park, NC: U.S. Department of Health and Human Services, Public Health Service, National Toxicology Program. http://ntpserver.niehs.nih.gov/ntp/roc/toc11.html. March 08, 2006.

Nusier M, Bataineh HN. 2005. Effect of boric acid on fertility, aggressiveness and sex behaviour in male rats. Asian J Chem 17(4):2579-2588.

Odunola OA. 1997. Individual and combined genotoxic response to boric acid and aflatoxin B1 in Escherichia coli PQ37. East Afr Med J 74(8):499-502.

Ordonez A, Loredo J, De Miguel E, et al. 2003. Distribution of heavy metals in the street dusts and soils of an industrial city in northern Spain. Arch Environ Contam Toxicol 44:160-170.

OSHA. 1989. Method ID216SG. Boron trifluoride (BF3). Occupational Safety and Health Administration. http://www.osha.gov/dts/sltc/methods/partial/id216sg/id216sg.html. March 14, 2007.

OSHA. 2006a. Air contaminants. Occupational safety and health standards for shipyard employment. Washington, DC: Occupational Safety and Health Administration. Code of Federal Regulations. 29 CFR 1915.1000. http://www.osha.gov/comp-links.html. April 11, 2007.

OSHA. 2006b. Gases, vapors, fumes, dusts, and mists. Safety and health regulations for construction. Washington, DC: Occupational Safety and Health Administration. Code of Federal Regulations. 29 CFR 1926.55, Appendix A. http://www.osha.gov/comp-links.html. April 11, 2007.

OSHA. 2006c. Limits for air contaminants. Occupational safety and health standards. Washington, DC: Occupational Safety and Health Administration. Code of Federal Regulations. 29 CFR 1910.1000. http://www.osha.gov/comp-links.html. April 11, 2007.

OSHA. 2006d. List of highly hazardous chemicals, toxics, and reactives. Occupational safety and health standards. Washington, DC: Occupational Safety and Health Administration. Code of Federal Regulations. 29 CFR 1910.119. http://www.osha.gov/comp-links.html. April 11, 2007. 


\section{REFERENCES}

O'Sullivan K, Taylor M. 1983. Chronic boric acid poisoning in infants. Arch Dis Child 58:737-739.

Owen GM, Brozek J. 1966. Influence of age, sex and nutrition on body composition during childhood and adolescence. In: Falkner F, ed. Human development. Philadelphia, PA: WB Saunders, 222-238.

Ozturkcan S, Dundar R, Katilmus H, et al. 2008. The ototoxic effect of boric acid solutions applied into the middle ear of guinea pigs. Eur Arch Otorhinolaryngol [epub ahead of print].

Pahl MV, Culver BD, Strong PL, et al. 2001. The effect of pregnancy on renal clearance of boron in humans: A study based on normal dietary intake of boron. Toxicol Sci 60(2):252-256.

Parks WL, White JL. 1952. Boron retention by clay and humus systems saturated with various cations. Soil Sci Soc Proc 16:298-300.

Price CJ, Marr MC, Myers CB, et al. 1996b. The developmental toxicity of boric acid in rabbits. Fundam Appl Toxicol 34(2):176-187.

Price CJ, Strong PL, Marr MC, et al. 1996a. Developmental toxicity NOAEL and postnatal recovery in rats fed boric acid during gestation. Fundam Appl Toxicol 32:179-193.

Price CJ, Strong PL, Murray FJ, et al. 1998. Developmental effects of boric acid in rats related to maternal blood boron concentrations. Biol Trace Elem Res 66:359-372.

Rai D, Zachara JM, Schwab AP, et al. 1986. Chemical attenuation rates, coefficients, and constants in leachate migration. Vol. 1. A critical review. Palo Alto, CA: Electric Power Research Institute, Research Project 2198-1.

Rainey CJ, Nyquist LA, Christensen RE, et al. 1999. Daily boron intake from the American diet. J Am Diet Assoc 99(3):335-340.

Rainey CJ, Nyquist LA, Coughlin JR, et al. 2002. Dietary boron intake in the United States: CSFII 1994-1996. J Food Comp Anal 15:237-250.

Restuccio A, Mortensen ME, Kelley MT. 1992. Fatal ingestion of boric acid in an adult. Am J Emerg Med 10(6):545-547.

Rope SK, Arthur WJ, Craig TH, et al. 1988. Nutrient and trace elements in soil and desert vegetation of southern Idaho. Environ Monit Assess 10:1-24.

Rosborg I, Nihlgard B, Gerhardsson L. 2003. Hair element concentrations in females in one acid and one alkaline area in southern Sweden. Ambio 32(7):440-446.

RTECS. 2007. Boron. Registry of Toxic Effects on Chemical Substances. National Institute of Occupational Safety and Health. MDL Information Systems, Inc. May 7, 2007.

Ruth JH. 1986. Odor threshold and irritation levels of several chemical substances: A review. Am Ind Hyg Assoc J 47:142-151.

Sah RN, Brown PH. 1997. Boron determination: A review of analytical methods. Microchem J 56:285304. 


\section{REFERENCES}

Sakata M. 1987. Relationship between adsorption of arsenic (III) and boron by soil and soil properties. Environ Sci Technol 21:1126-1130.

Sayli BS. 1998a. An assessment of fertility in boron-exposed Turkish subpopulations. Evidence that boron has no effect on human reproduction. Biol Trace Elem Res 66:409-422.

Sayli BS. 1998b. The sex ratio of offspring of people exposed to boron. Reprod Toxicol 12(6):673-674.

Sayli BS. 2003. Low frequency of infertility among workers in a borate processing facility. Biol Trace Elem Res 93:19-29.

Sayli BS, Tuccar E, Elhan AH. 1998. An assessment of fertility in boron-exposed Turkish subpopulations. Reprod Toxicol 12(3):297-304.

Schillinger BM, Berstein M, Goldberg LA, et al. 1982. Boric acid poisoning. J Am Acad Dermatol 7:667-673.

Schou JS, Jansen JA, Aggerbeck B. 1984. Human pharmacokinetics and safety of boric acid. Arch Toxicol Suppl 7:232-235.

Schubert DM, Brotherton, RJ. 2006. Boron: Inorganic chemistry. In: Encyclopedia of inorganic chemistry. John Wiley \& Sons, Inc.

http://mrw.interscience.wiley.com/emrw/9780470862100/eic/article/ia025/current/pdf. March 11, 2009.

Seal BS, Weeth HJ. 1980. Effect of boron in drinking water on the male laboratory rat. Bull Environ Contam Toxicol 25:782-789.

Setchell BP, Waites GMH. 1975. The blood-testis barrier. In: Creep RO, Astwood EB, Geiger SR, eds. Handbook of physiology: Endocrinology V. Washington, DC: American Physiological Society, 143172.

Settimi L, Elovaara E, Savolainen H. 1982. Effects of extended peroral borate ingestion on rat liver and brain. Toxicol Lett 10:219-223.

Sittig M. 1985. Handbook of toxic and hazardous chemicals and carcinogens. 2nd ed. Park Ridge, NJ: Noyes Publications, 137-142.

Smyth HF, Carpenter CP, Weil CS, et al. 1969. Range-finding toxicity data: List VII. Am Ind Hyg Assoc J 30:470-476.

SRI. 2008. 2008 Directory of chemical producers. Menlo Park, CA: SRI Consulting, 453, 851.

Stewart NJ, McHugh TP. 1990. B. Borates. In: Haddad LM, Winchester JF, eds. Clinical management of poisoning and drug overdose. 2nd ed. Philadelphia, PA: W.B. Saunders Company, 1447-1451.

Stokinger HE. 1981. Boron. In: Clayton GD, Clayton FE, eds. Patty's industrial hygiene and toxicology. Vol. 2B. Toxicology. 3rd ed. New York, NY: John Wiley and Sons, 2978-3005.

Sturgiss SN, Wilkinson R, Davison JM. 1996. Renal reserve during human pregnancy. Am J Physiol 271(40):F16-F20. 


\section{REFERENCES}

Tagawa T, Kono K, Dote T, et al. 2000. Pharmacokinetics and effects after intravenous administration of high-dose boron to rat. Int Arch Occup Environ Health 73:S98-S100.

Takahashi T, Yawata S, Hoshino H. 2008. Determination of boron in water samples at nanograms per cubic decimeter levels by reversed-phase partition high performance liquid chromatography with pecolumn complexation reaction using salicylaldehyde and 1-amino-8-naphthol-3,6-disulfonate. Anal Bioanal Chem 391:1101-1106.

Tarasenko NY, Kaspurov AA, Strongina OM. 1972. [Effect of boric acid on the generative function in males.] Gigirna Truda i Professionalyne Zabolevaniya 11:13-16. (Russian)

Thomas K, Colborn T. 1992. Organochlorine endocrine disruptors in human tissue. In: Colborn T, Clement C, eds. Chemically induced alterations in sexual and functional development: The wildlife/human connection. Princeton, NJ: Princeton Scientific Publishing, 365-394.

Thompson SE, Burton CA, Quinn DJ, et al. 1972. Concentration factors of chemical elements in edible aquatic organisms. Livermore, CA: Lawrence Livermore Laboratory, Bio-Medical Division, University of California. UCRL50564REV1.

Treinen KA, Chapin RE. 1991. Development of testicular lesions in F344 rats after treatment with boric acid. Toxicol Appl Pharmacol 107:325-335.

TRI06. 2008. TRI explorer: Providing access to EPA's toxics release inventory data. Washington, DC: Office of Information Analysis and Access. Office of Environmental Information. U.S. Environmental Protection Agency. Toxics Release Inventory. http://www.epa.gov/triexplorer/. February 12, 2009.

Tsui PT, McCart PJ. 1981. Chlorinated hydrocarbon residues and heavy metals in several fish species from the Cold Lake area in Alberta, Canada. Intern J Environ Anal Chem 10:277-285.

Tuccar E, Elhan AH, Yaduz Y, et al. 1998. Comparison of infertility rates in communities from boronrich and boron-poor territories. Biol Trace Elem Res 66:401-407.

Uemura T, Omae K, Nakashima H, et al. 1995. Acute and subacute inhalation toxicity of diborane in male ICR mice. Arch Toxicol 69(6):397-404.

USGS. 1984. Element concentrations in soils and other surficial materials of the conterminous United States. Alexandria, VA: U.S. Geological Survey. Geological Survey Professional Paper 1270.

USGS. 1989. In: Fishman MJ, Friedman LC, eds. Techniques of water-resources investigations, Book 5, Chapter A1. Methods for determination of inorganic substances in water and fluvial sediments. Washington, DC: U.S. Geological Survey. http://pubs.usgs.gov/twri/twri5-a1/pdf/TWRI_5-A1.pdf. April 12, 2007.

USGS. 1996a. Methods of analysis by the U.S. Geological Survey National Water Quality LaboratoryPreparation procedure for aquatic biological material determined for trace metals. Denver, CO: U.S. Geological Survey. Open-File Report 96-362. http://pubs.er.usgs.gov/usgspubs/ofr/ofr96362. March 26, 2007.

USGS. 1996b. Methods of analysis by the U.S. Geological Survey National Water Quality LaboratoryDetermination of dissolved aluminum and boron in water by inductively coupled plasma-atomic emission 
spectrometry. Denver, CO: U.S. Geological Survey. Open-File Report 96-149. http://pubs.er.usgs.gov/usgspubs/ofr/ofr96149. March 26, 2007.

USGS. 1998. Methods of analysis by the U.S. Geological Survey National Water Quality LaboratoryDetermination of elements in whole-water digests using inductively coupled plasma-optical emission spectrometry and inductively coupled plasma-mass spectrometry. Denver, CO: U.S. Geological Survey. Open-File Report 98-165. http://pubs.er.usgs.gov/usgspubs/ofr/ofr98165. March 26, 2007.

USGS. 1999. Methods of analysis by the U.S. Geological Survey National Water Quality LaboratoryDetermination of dissolved arsenic, boron, lithium, selenium, strontium, thallium, and vanadium using inductively coupled plasma-mass spectrometry. Denver, CO: U.S. Geological Survey. Open-File Report 99-093. http://pubs.er.usgs.gov/usgspubs/ofr/ofr99093. March 26, 2007.

USGS. 2008. Boron. 2007 minerals yearbook. U.S. Geological Survey, 13-1 to 13-10.

http://minerals.usgs.gov/minerals/pubs/commodity/boron/boronmyb05.pdf. March 31, 2009.

USGS. 2009. Boron. Mineral commodity studies. U.S. Geological Survey, 34-35.

http://minerals.usgs.gov/minerals/pubs/commodity/boron/mcs-2009-boron.pdf. March 31, 2009.

Usuda K, Kono K, Dote T, et al. 1998. Study on urine boron reference values of Japanese men: Use of confidence intervals as an indicator of exposure to boron compounds. Sci Total Environ 220(1):45-53.

Vanderpool RA, Hoff D, Johnson PE. 1994. Use of inductively coupled plasma-mass spectrometry in boron-10 stable isotope experiments with plants, rats, and humans. Environ Health Perspect 102(Suppl 7):13-20.

Van Ormer DG. 1975. Atomic absorption analysis of some trace elements of toxicological interest. J Forensic Sci 20:595-623.

Vaziri ND, Oveisi F, Culver BD, et al. 2001. The effect of pregnancy on renal clearance of boron in rats given boric acid orally. Toxicol Sci 60:257-263.

Vengosh A, Heumann KG, Juraske S, et al. 1994. Boron isotope applications for tracing sources of contamination in groundwater. Environ Sci Technol 28(11):1968-1974.

Viccellio P, Bania T, Brent J, et al., eds. 1998. Emergency toxicology. 2nd ed. Philadelphia, PA: Lippincott-Raven, 448-449, 470, 1141.

Vieira I, Sonnier M, Cresteil T. 1996. Developmental expression of CYP2E1 in the human liver: Hypermethylation control of gene expression during the neonatal period. Eur J Biochem 238(2):476-483.

Vignec AJ, Ellis R. 1954. Inabsorbability of boric acid in infant powder. Am J Dis Child 88:72-80.

Waggott A. 1969. An investigation of the potential problem of increasing boron concentrations in rivers and water courses. Water Res 3:749-765.

Wegman DH, Eisen EA, Hu X, et al. 1994. Acute and chronic respiratory effects of sodium borate particulate exposures. Environ Health Perspect (Suppl 7):102:119-128.

Weir RJ, Fisher RS. 1972. Toxicologic studies on borax and boric acid. Toxicol Appl Pharmacol 23:351-364. 
Wery N, Narotsky MG, Pacico N, et al. 2003. Defects in cervical vertebrae in boric acid-exposed rat embryos are associated with anterior shifts of hox gene expression domains. Birth Defects Res A Clin Mol Teratol 67(1):59-67.

West JR, Smith HW, Chasis H. 1948. Glomerular filtration rate, effective renal blood flow, and maximal tubular excretory capacity in infancy. J Pediatr 32:10-18.

Wester RC, Hui X, Hartway T, et al. 1998. In vivo percutaneous absorption of boric acid, borax, and disodium octaborate tetrahydrate in humans compared to in vitro absoption in human skin from infinite and finite doses. Toxicol Sci 45:42-51.

WHO. 2000. Air quality guidelines. 2nd ed. Geneva, Switzerland: World Health Organization. http://www.euro.who.int/Document/AIQ/AirQualRepMtg.pdf. March 08, 2006.

WHO. 2004. Guidelines for drinking-water quality. Vol. 1. Recommendations. 3rd ed. Geneva, Switzerland: World Health Organization. http://www.who.int/water_sanitation_health/dwq/gdwq3/en/. March 08, 2006.

Whorton D, Haas J, Trent L. 1994. Reproductive effects of inorganic borates on male employees: Birth rate assessment. Environ Health Perspect Suppl 102(7):129-132.

Widdowson EM, Dickerson JWT. 1964. Chemical composition of the body. In: Comar CL, Bronner F, eds. Mineral metabolism: An advanced treatise. Vol. II: The elements. Part A. New York, NY: Academic Press, 1-247.

Wilding JL, Smith WJ, Yevich P, et al. 1959. The toxicity of boron oxide. Am Ind Hyg Assoc J 20:284289.

Wittig A, Michel J, Moss RL, et al. 2008. Boron analysis and boron imaging in biological materials for Boron Neutron Capture Therapy (BNCT). Crit Rev Oncol Hematol 68(1):66-90.

Wong LC, Heimbach MD, Truscott DR, et al. 1964. Boric acid poisoning: Report of 11 cases. Can Med Assoc J 90:1018-1023.

Woskie SR, Shen P, Eisen EA, et al. 1994. The real-time dust exposure of sodium borate workers: Examination of exposure variability. Am Ind Hyg Assoc J 55(3):207-217.

Xiao YK, Li SZ, Wei HZ, et al. 2007. Boron isotopic fractionation during seawater evaporation. Mar Chem 103(3-4):382-392.

Xing X, Wu G, Wei F, et al. 2008. Biomarkers of environmental and workplace boron exposure. J Occup Environ Hyg 5:141-147.

Yoshizaki H, Izumi Y, Hirayama C, et al. 1999. Availability of sperm examination for male reproductive toxicities in rats treated with boric acid. J Toxicol Sci 24(3):199-208.

Ziegler EE, Edwards BB, Jensen RL, et al. 1978. Absorption and retention of lead by infants. Pediatr Res 12(1):29-34. 


\section{GLOSSARY}

Absorption-The taking up of liquids by solids, or of gases by solids or liquids.

Acute Exposure-Exposure to a chemical for a duration of 14 days or less, as specified in the Toxicological Profiles.

Adsorption-The adhesion in an extremely thin layer of molecules (as of gases, solutes, or liquids) to the surfaces of solid bodies or liquids with which they are in contact.

Adsorption Coefficient $\left(\mathbf{K}_{\mathbf{o c}}\right)$ - The ratio of the amount of a chemical adsorbed per unit weight of organic carbon in the soil or sediment to the concentration of the chemical in solution at equilibrium.

Adsorption Ratio (Kd) — The amount of a chemical adsorbed by sediment or soil (i.e., the solid phase) divided by the amount of chemical in the solution phase, which is in equilibrium with the solid phase, at a fixed solid/solution ratio. It is generally expressed in micrograms of chemical sorbed per gram of soil or sediment.

Benchmark Dose (BMD) - The dose expected to result in a specified change of a biological effect (the benchmark response, or BMR), generally $1 \%$ to $10 \%$ from the untreated population. The BMD is determined by modeling the dose response data in the region of the dose response relationship where biologically observable data are feasible.

Benchmark Dose, Lower Limit (BMDL)—The lower confidence limit on the benchmark dose. For example, a BMDL $_{10}$ would be the dose at the $95 \%$ lower confidence limit on a $10 \%$ response, and the benchmark response (BMR) would be $10 \%$.

Benchmark Dose Model—A statistical dose-response model applied to either experimental toxicological or epidemiological data to calculate a BMD.

Bioconcentration Factor (BCF) - The quotient of the concentration of a chemical in aquatic organisms at a specific time or during a discrete time period of exposure divided by the concentration in the surrounding water at the same time or during the same period.

Biomarkers-Broadly defined as indicators signaling events in biologic systems or samples. They have been classified as markers of exposure, markers of effect, and markers of susceptibility.

Cancer Effect Level (CEL) - The lowest dose of chemical in a study, or group of studies, that produces significant increases in the incidence of cancer (or tumors) between the exposed population and its appropriate control.

Carcinogen-A chemical capable of inducing cancer.

Case-Control Study - A type of epidemiological study that examines the relationship between a particular outcome (disease or condition) and a variety of potential causative agents (such as toxic chemicals). In a case-controlled study, a group of people with a specified and well-defined outcome is identified and compared to a similar group of people without outcome.

Case Report-Describes a single individual with a particular disease or exposure. These may suggest some potential topics for scientific research, but are not actual research studies. 
Case Series-Describes the experience of a small number of individuals with the same disease or exposure. These may suggest potential topics for scientific research, but are not actual research studies.

Ceiling Value-A concentration of a substance that should not be exceeded, even instantaneously.

Chronic Exposure-Exposure to a chemical for 365 days or more, as specified in the Toxicological Profiles.

Cohort Study-A type of epidemiological study of a specific group or groups of people who have had a common insult (e.g., exposure to an agent suspected of causing disease or a common disease) and are followed forward from exposure to outcome. At least one exposed group is compared to one unexposed group.

Cross-sectional Study-A type of epidemiological study of a group or groups of people that examines the relationship between exposure and outcome to a chemical or to chemicals at one point in time.

Data Needs-Substance-specific informational needs that if met would reduce the uncertainties of human health assessment.

Developmental Toxicity-The occurrence of adverse effects on the developing organism that may result from exposure to a chemical prior to conception (either parent), during prenatal development, or postnatally to the time of sexual maturation. Adverse developmental effects may be detected at any point in the life span of the organism.

Dose-Response Relationship-The quantitative relationship between the amount of exposure to a toxicant and the incidence of the adverse effects.

Embryotoxicity and Fetotoxicity-Any toxic effect on the conceptus as a result of prenatal exposure to a chemical; the distinguishing feature between the two terms is the stage of development during which the insult occurs. The terms, as used here, include malformations and variations, altered growth, and in utero death.

Environmental Protection Agency (EPA) Health Advisory-An estimate of acceptable drinking water levels for a chemical substance based on health effects information. A health advisory is not a legally enforceable federal standard, but serves as technical guidance to assist federal, state, and local officials.

Epidemiology-Refers to the investigation of factors that determine the frequency and distribution of disease or other health-related conditions within a defined human population during a specified period.

Genotoxicity-A specific adverse effect on the genome of living cells that, upon the duplication of affected cells, can be expressed as a mutagenic, clastogenic, or carcinogenic event because of specific alteration of the molecular structure of the genome.

Half-life-A measure of rate for the time required to eliminate one half of a quantity of a chemical from the body or environmental media.

Immediately Dangerous to Life or Health (IDLH) - The maximum environmental concentration of a contaminant from which one could escape within 30 minutes without any escape-impairing symptoms or irreversible health effects. 
Immunologic Toxicity-The occurrence of adverse effects on the immune system that may result from exposure to environmental agents such as chemicals.

Immunological Effects—Functional changes in the immune response.

Incidence-The ratio of individuals in a population who develop a specified condition to the total number of individuals in that population who could have developed that condition in a specified time period.

Intermediate Exposure-Exposure to a chemical for a duration of 15-364 days, as specified in the Toxicological Profiles.

In Vitro-Isolated from the living organism and artificially maintained, as in a test tube.

In Vivo-Occurring within the living organism.

Lethal Concentration $(\mathbf{L O})\left(\mathbf{L C}_{\mathbf{L O}}\right)$ - The lowest concentration of a chemical in air that has been reported to have caused death in humans or animals.

Lethal Concentration ${ }_{(50)}\left(\mathbf{L C}_{50}\right)$ - A calculated concentration of a chemical in air to which exposure for a specific length of time is expected to cause death in $50 \%$ of a defined experimental animal population.

Lethal Dose $_{(\mathbf{L O})}\left(\mathbf{L D}_{\mathbf{L o}}\right)$ - The lowest dose of a chemical introduced by a route other than inhalation that has been reported to have caused death in humans or animals.

Lethal Dose $\mathbf{S}_{(\mathbf{5 0})}\left(\mathbf{L D}_{\mathbf{5 0}}\right)$ - The dose of a chemical that has been calculated to cause death in $50 \%$ of a defined experimental animal population.

Lethal Time $\mathbf{( 5 0 )}_{(\mathbf{S 0}}\left(\mathbf{L T}_{\mathbf{5 0}}\right)$ - A calculated period of time within which a specific concentration of a chemical is expected to cause death in $50 \%$ of a defined experimental animal population.

Lowest-Observed-Adverse-Effect Level (LOAEL) — The lowest exposure level of chemical in a study, or group of studies, that produces statistically or biologically significant increases in frequency or severity of adverse effects between the exposed population and its appropriate control.

Lymphoreticular Effects-Represent morphological effects involving lymphatic tissues such as the lymph nodes, spleen, and thymus.

Malformations-Permanent structural changes that may adversely affect survival, development, or function.

Minimal Risk Level (MRL) — An estimate of daily human exposure to a hazardous substance that is likely to be without an appreciable risk of adverse noncancer health effects over a specified route and duration of exposure.

Modifying Factor (MF) —A value (greater than zero) that is applied to the derivation of a Minimal Risk Level (MRL) to reflect additional concerns about the database that are not covered by the uncertainty factors. The default value for a MF is 1 .

Morbidity-State of being diseased; morbidity rate is the incidence or prevalence of disease in a specific population. 
Mortality-Death; mortality rate is a measure of the number of deaths in a population during a specified interval of time.

Mutagen-A substance that causes mutations. A mutation is a change in the DNA sequence of a cell's DNA. Mutations can lead to birth defects, miscarriages, or cancer.

Necropsy - The gross examination of the organs and tissues of a dead body to determine the cause of death or pathological conditions.

Neurotoxicity-The occurrence of adverse effects on the nervous system following exposure to a chemical.

No-Observed-Adverse-Effect Level (NOAEL) - The dose of a chemical at which there were no statistically or biologically significant increases in frequency or severity of adverse effects seen between the exposed population and its appropriate control. Effects may be produced at this dose, but they are not considered to be adverse.

Octanol-Water Partition Coefficient $\left(\mathbf{K}_{\mathbf{o w}}\right)$ - The equilibrium ratio of the concentrations of a chemical in $n$-octanol and water, in dilute solution.

Odds Ratio (OR) - A means of measuring the association between an exposure (such as toxic substances and a disease or condition) that represents the best estimate of relative risk (risk as a ratio of the incidence among subjects exposed to a particular risk factor divided by the incidence among subjects who were not exposed to the risk factor). An OR of greater than 1 is considered to indicate greater risk of disease in the exposed group compared to the unexposed group.

Organophosphate or Organophosphorus Compound-A phosphorus-containing organic compound and especially a pesticide that acts by inhibiting cholinesterase.

Permissible Exposure Limit (PEL)—An Occupational Safety and Health Administration (OSHA) allowable exposure level in workplace air averaged over an 8-hour shift of a 40-hour workweek.

Pesticide-General classification of chemicals specifically developed and produced for use in the control of agricultural and public health pests.

Pharmacokinetics - The dynamic behavior of a material in the body, used to predict the fate (disposition) of an exogenous substance in an organism. Utilizing computational techniques, it provides the means of studying the absorption, distribution, metabolism, and excretion of chemicals by the body.

Pharmacokinetic Model—A set of equations that can be used to describe the time course of a parent chemical or metabolite in an animal system. There are two types of pharmacokinetic models: data-based and physiologically-based. A data-based model divides the animal system into a series of compartments, which, in general, do not represent real, identifiable anatomic regions of the body, whereas the physiologically-based model compartments represent real anatomic regions of the body.

Physiologically Based Pharmacodynamic (PBPD) Model-A type of physiologically based doseresponse model that quantitatively describes the relationship between target tissue dose and toxic end points. These models advance the importance of physiologically based models in that they clearly describe the biological effect (response) produced by the system following exposure to an exogenous substance. 
Physiologically Based Pharmacokinetic (PBPK) Model—Comprised of a series of compartments representing organs or tissue groups with realistic weights and blood flows. These models require a variety of physiological information: tissue volumes, blood flow rates to tissues, cardiac output, alveolar ventilation rates, and possibly membrane permeabilities. The models also utilize biochemical information, such as air/blood partition coefficients, and metabolic parameters. PBPK models are also called biologically based tissue dosimetry models.

Prevalence-The number of cases of a disease or condition in a population at one point in time.

Prospective Study - A type of cohort study in which the pertinent observations are made on events occurring after the start of the study. A group is followed over time.

$\mathbf{q}_{1}{ }^{*}$-The upper-bound estimate of the low-dose slope of the dose-response curve as determined by the multistage procedure. The $\mathrm{q}_{1}{ }^{*}$ can be used to calculate an estimate of carcinogenic potency, the incremental excess cancer risk per unit of exposure (usually $\mu \mathrm{g} / \mathrm{L}$ for water, $\mathrm{mg} / \mathrm{kg} /$ day for food, and $\mu \mathrm{g} / \mathrm{m}^{3}$ for air).

Recommended Exposure Limit (REL) — A National Institute for Occupational Safety and Health (NIOSH) time-weighted average (TWA) concentration for up to a 10-hour workday during a 40-hour workweek.

Reference Concentration (RfC)—An estimate (with uncertainty spanning perhaps an order of magnitude) of a continuous inhalation exposure to the human population (including sensitive subgroups) that is likely to be without an appreciable risk of deleterious noncancer health effects during a lifetime. The inhalation reference concentration is for continuous inhalation exposures and is appropriately expressed in units of $\mathrm{mg} / \mathrm{m}^{3}$ or $\mathrm{ppm}$.

Reference Dose (RfD)—An estimate (with uncertainty spanning perhaps an order of magnitude) of the daily exposure of the human population to a potential hazard that is likely to be without risk of deleterious effects during a lifetime. The RfD is operationally derived from the no-observed-adverse-effect level (NOAEL, from animal and human studies) by a consistent application of uncertainty factors that reflect various types of data used to estimate RfDs and an additional modifying factor, which is based on a professional judgment of the entire database on the chemical. The RfDs are not applicable to nonthreshold effects such as cancer.

Reportable Quantity (RQ) - The quantity of a hazardous substance that is considered reportable under the Comprehensive Environmental Response, Compensation, and Liability Act (CERCLA). Reportable quantities are (1) 1 pound or greater or (2) for selected substances, an amount established by regulation either under CERCLA or under Section 311 of the Clean Water Act. Quantities are measured over a 24-hour period.

Reproductive Toxicity-The occurrence of adverse effects on the reproductive system that may result from exposure to a chemical. The toxicity may be directed to the reproductive organs and/or the related endocrine system. The manifestation of such toxicity may be noted as alterations in sexual behavior, fertility, pregnancy outcomes, or modifications in other functions that are dependent on the integrity of this system. 
Retrospective Study-A type of cohort study based on a group of persons known to have been exposed at some time in the past. Data are collected from routinely recorded events, up to the time the study is undertaken. Retrospective studies are limited to causal factors that can be ascertained from existing records and/or examining survivors of the cohort.

Risk-The possibility or chance that some adverse effect will result from a given exposure to a chemical.

Risk Factor-An aspect of personal behavior or lifestyle, an environmental exposure, or an inborn or inherited characteristic that is associated with an increased occurrence of disease or other health-related event or condition.

Risk Ratio-The ratio of the risk among persons with specific risk factors compared to the risk among persons without risk factors. A risk ratio greater than 1 indicates greater risk of disease in the exposed group compared to the unexposed group.

Short-Term Exposure Limit (STEL) - The American Conference of Governmental Industrial Hygienists (ACGIH) maximum concentration to which workers can be exposed for up to 15 minutes continually. No more than four excursions are allowed per day, and there must be at least 60 minutes between exposure periods. The daily Threshold Limit Value-Time Weighted Average (TLV-TWA) may not be exceeded.

Standardized Mortality Ratio (SMR) - A ratio of the observed number of deaths and the expected number of deaths in a specific standard population.

Target Organ Toxicity-This term covers a broad range of adverse effects on target organs or physiological systems (e.g., renal, cardiovascular) extending from those arising through a single limited exposure to those assumed over a lifetime of exposure to a chemical.

Teratogen—A chemical that causes structural defects that affect the development of an organism.

Threshold Limit Value (TLV)—An American Conference of Governmental Industrial Hygienists (ACGIH) concentration of a substance to which most workers can be exposed without adverse effect. The TLV may be expressed as a Time Weighted Average (TWA), as a Short-Term Exposure Limit (STEL), or as a ceiling limit (CL).

Time-Weighted Average (TWA)_An allowable exposure concentration averaged over a normal 8-hour workday or 40-hour workweek.

Toxic Dose $\mathbf{( 5 0 )}_{(\mathbf{T})} \mathbf{T D}_{\mathbf{5 0}}$ - - A calculated dose of a chemical, introduced by a route other than inhalation, which is expected to cause a specific toxic effect in $50 \%$ of a defined experimental animal population.

Toxicokinetic - The absorption, distribution, and elimination of toxic compounds in the living organism. 
Uncertainty Factor (UF) — A factor used in operationally deriving the Minimal Risk Level (MRL) or Reference Dose (RfD) or Reference Concentration (RfC) from experimental data. UFs are intended to account for (1) the variation in sensitivity among the members of the human population, (2) the uncertainty in extrapolating animal data to the case of human, (3) the uncertainty in extrapolating from data obtained in a study that is of less than lifetime exposure, and (4) the uncertainty in using lowestobserved-adverse-effect level (LOAEL) data rather than no-observed-adverse-effect level (NOAEL) data. A default for each individual UF is 10; if complete certainty in data exists, a value of 1 can be used; however, a reduced UF of 3 may be used on a case-by-case basis, 3 being the approximate logarithmic average of 10 and 1 (see for example, Dourson, 1994).

Xenobiotic-Any chemical that is foreign to the biological system. 


\section{APPENDIX A. ATSDR MINIMAL RISK LEVELS AND WORKSHEETS}

The Comprehensive Environmental Response, Compensation, and Liability Act (CERCLA) [42 U.S.C. 9601 et seq.], as amended by the Superfund Amendments and Reauthorization Act (SARA) [Pub. L. 99499], requires that the Agency for Toxic Substances and Disease Registry (ATSDR) develop jointly with the U.S. Environmental Protection Agency (EPA), in order of priority, a list of hazardous substances most commonly found at facilities on the CERCLA National Priorities List (NPL); prepare toxicological profiles for each substance included on the priority list of hazardous substances; and assure the initiation of a research program to fill identified data needs associated with the substances.

The toxicological profiles include an examination, summary, and interpretation of available toxicological information and epidemiologic evaluations of a hazardous substance. During the development of toxicological profiles, Minimal Risk Levels (MRLs) are derived when reliable and sufficient data exist to identify the target organ(s) of effect or the most sensitive health effect(s) for a specific duration for a given route of exposure. An MRL is an estimate of the daily human exposure to a hazardous substance that is likely to be without appreciable risk of adverse noncancer health effects over a specified duration of exposure. MRLs are based on noncancer health effects only and are not based on a consideration of cancer effects. These substance-specific estimates, which are intended to serve as screening levels, are used by ATSDR health assessors to identify contaminants and potential health effects that may be of concern at hazardous waste sites. It is important to note that MRLs are not intended to define clean-up or action levels.

MRLs are derived for hazardous substances using the no-observed-adverse-effect level/uncertainty factor approach. They are below levels that might cause adverse health effects in the people most sensitive to such chemical-induced effects. MRLs are derived for acute (1-14 days), intermediate (15-364 days), and chronic (365 days and longer) durations and for the oral and inhalation routes of exposure. Currently, MRLs for the dermal route of exposure are not derived because ATSDR has not yet identified a method suitable for this route of exposure. MRLs are generally based on the most sensitive chemical-induced end point considered to be of relevance to humans. Serious health effects (such as irreparable damage to the liver or kidneys, or birth defects) are not used as a basis for establishing MRLs. Exposure to a level above the MRL does not mean that adverse health effects will occur.

MRLs are intended only to serve as a screening tool to help public health professionals decide where to look more closely. They may also be viewed as a mechanism to identify those hazardous waste sites that 
are not expected to cause adverse health effects. Most MRLs contain a degree of uncertainty because of the lack of precise toxicological information on the people who might be most sensitive (e.g., infants, elderly, nutritionally or immunologically compromised) to the effects of hazardous substances. ATSDR uses a conservative (i.e., protective) approach to address this uncertainty consistent with the public health principle of prevention. Although human data are preferred, MRLs often must be based on animal studies because relevant human studies are lacking. In the absence of evidence to the contrary, ATSDR assumes that humans are more sensitive to the effects of hazardous substance than animals and that certain persons may be particularly sensitive. Thus, the resulting MRL may be as much as 100 -fold below levels that have been shown to be nontoxic in laboratory animals.

Proposed MRLs undergo a rigorous review process: Health Effects/MRL Workgroup reviews within the Division of Toxicology, expert panel peer reviews, and agency-wide MRL Workgroup reviews, with participation from other federal agencies and comments from the public. They are subject to change as new information becomes available concomitant with updating the toxicological profiles. Thus, MRLs in the most recent toxicological profiles supersede previously published levels. For additional information regarding MRLs, please contact the Division of Toxicology and Environmental Medicine, Agency for Toxic Substances and Disease Registry, 1600 Clifton Road NE, Mailstop F-62, Atlanta, Georgia 30333. 


\section{MINIMAL RISK LEVEL (MRL) WORKSHEET}

$\begin{array}{ll}\text { Chemical Name: } & \text { Boron and Compounds } \\ \text { CAS Number: } & 7440-42-8 \\ \text { Date: } & \text { August 2009 } \\ \text { Profile Status: } & \text { Final Draft Post-Public Comment } \\ \text { Route: } & \text { [X] Inhalation [ ] Oral } \\ \text { Duration: } & \text { [X] Acute [ ] Intermediate [ ] Chronic } \\ \text { Graph Key: } & 2 \\ \text { Species: } & \text { Human }\end{array}$

\section{Minimal Risk Level: $0.3[\mathrm{X}] \mathrm{mg} / \mathrm{m}^{3} \quad$ [ ] ppm}

Reference: Cain WS, Jalowayski AA, Kleinman M, et al. 2004. Sensory and associated reactions to mineral dusts: Sodium borate, calcium oxide, and calcium sulfate. J Occup Environ Hyg 1:222-236.

Cain WS, Jalowayski AA, Schmidt R, et al. 2008. Chemesthetic responses to airborne mineral dusts: boric acid compared to alkaline materials. Int Arch Occup Environ Health 81:337-345.

Experimental design: Male and female volunteers were trained to recognize the difference in chemesthetic feel (pungency or irritancy) of various levels of $\mathrm{CO}_{2}$ offered to the eyes, nose, and throat. Exposures of $\geq 17.7 \% \mathrm{CO}_{2}$ resulted in a "feel" described by the volunteers as irritating. Twelve male volunteers were exposed to $0,0.8,1.5,3.0,4.5$, or $6.0 \mathrm{mg}$ boron $/ \mathrm{m}^{3}(0,5,10,20,30$, or $40 \mathrm{mg}$ sodium borate pentahydrate/ $\mathrm{m}^{3}$ ) for 20 minutes while performing light exercise (Cain et al. 2004). At 5-minute intervals, they reported the magnitude of "feel" of borate dust in terms of equivalent $\mathrm{CO}_{2}$ irritancy. They were also observed for changes in nasal secretions (by mass) and nasal airway resistance relative to preexposure measurements. Data were reported as means of all responses at each concentration and time point.

In a similar fashion, six male and six female volunteers were exposed to $1.5 \mathrm{mg}$ boron $/ \mathrm{m}^{3}$ (10 mg sodium borate $/ \mathrm{m}^{3}$ ) or $1.8 \mathrm{mg}$ boron $/ \mathrm{m}^{3}\left(10 \mathrm{mg}\right.$ boric acid $\left./ \mathrm{m}^{3}\right)$ for 47 minutes while exercising. They reported the magnitude of "feel" of boric acid and borate dusts in terms of equivalent $\mathrm{CO}_{2}$ irritancy. They were also observed for changes in nasal secretions (by mass), nasal airway resistance, and respiration frequency.

Effects noted in study and corresponding doses: Male volunteers exposed to $\leq 3.0 \mathrm{mg}$ boron $/ \mathrm{m}^{3}$ ( $\leq 20 \mathrm{mg}$ sodium borate $/ \mathrm{m}^{3}$ ) for 20 minutes reported increasingly higher magnitude of feel of the dust in eyes, nose, and throat. However, the mean reported level of feel, compared to equivalent $\mathrm{CO}_{2}$ levels, was not considered irritating by the study subjects. The mean reported perception of feel at $\geq 4.5 \mathrm{mg}$ boron $/ \mathrm{m}^{3}$ ( $\geq 30$ sodium borate $/ \mathrm{m}^{3}$ ) was reported to feel irritating to the nose (Cain et al. 2004). Significantly increased nasal secretions (by mass) occurred at $1.5 \mathrm{mg}$ boron $/ \mathrm{m}^{3}$ (10 mg sodium borate $/ \mathrm{m}^{3}$ ), but not $0.8 \mathrm{mg}$ boron $/ \mathrm{m}^{3}$ (5 mg sodium borate $/ \mathrm{m}^{3}$ ) (Cain et al. 2004). Similarly, male and female volunteers exposed to $1.5 \mathrm{mg}$ boron $/ \mathrm{m}^{3}$ (10 mg sodium borate $/ \mathrm{m}^{3}$ ) or $1.8 \mathrm{mg}$ boron $/ \mathrm{m}^{3}$ (10 mg boric acid $\left./ \mathrm{m}^{3}\right)$ for 47 minutes while exercising reported a mean sense of feel that initially increased and peaked at the equivalent of slightly $<17.7 \% \mathrm{CO}_{2}$. Boron exposures with a mean reported feel equivalent to less than $17.7 \% \mathrm{CO}_{2}$ are considered non-irritating, although increases in nasal secretions were observed at $1.8 \mathrm{mg}$ boron $/ \mathrm{mg}^{3}$ (10 mg boric acid $/ \mathrm{m}^{3}$ ), but not at $0.9 \mathrm{mg}$ boron $/ \mathrm{mg}^{3}$ ( $5 \mathrm{mg}$ boric acid $/ \mathrm{m}^{3}$ ) (Cain et al. 2008). Nasal airway resistance decreased, then increased, as a result of exercise, but did not change in a doserelated manner. 
Dose and end point used for MRL derivation: A NOAEL of $0.8 \mathrm{mg} / \mathrm{m}^{3}$ associated with a minimal LOAEL of $1.5 \mathrm{mg} / \mathrm{m}^{3}$ for significantly increased volume of nasal secretions.

\section{[X] NOAEL [ ] LOAEL}

\section{Uncertainty Factors used in MRL derivation:}

\section{[ ] 10 for use of a LOAEL}

[ ] 10 for extrapolation from animals to humans

[X] 3 for human variability

Was a conversion used from ppm in food or water to a mg/body weight dose? No.

If an inhalation study in animals, list the conversion factors used in determining human equivalent dose: Not applicable.

Other additional studies or pertinent information that lend support to this MRL: In an early crosssectional study of sodium borate workers, past occurrence of symptoms of respiratory irritation such as dryness of the mouth, nose, or throat, dry cough, nose bleeds, and sore throat were reported at elevated frequencies in workers in areas with mean dust concentrations of 8.4 and $14.6 \mathrm{mg}$ particulates $/ \mathrm{m}^{3}(1.8$ and $3.1 \mathrm{mg}$ boron $/ \mathrm{m}^{3}$, respectively), compared with workers in areas with lower mean dust levels of 4.0 and $1.1 \mathrm{mg}$ particulate $/ \mathrm{m}^{3}\left(0.9\right.$ and $0.2 \mathrm{mg}$ boron $\left./ \mathrm{m}^{3}\right)$ (Garabrant et al. 1984, 1985). In addition, a reduction in forced expiratory volume in 1 second $\left(\mathrm{FEV}_{1}\right)$ was measured in a subgroup of smoking workers with estimated high cumulative exposure ( $\geq 80 \mathrm{mg}$ particulate $/ \mathrm{m}^{3}, \geq 9 \mathrm{mg}$ boron $/ \mathrm{m}^{3}$ ) to sodium borate dusts, but not in groups of less-exposed smoking workers or in nonsmoking workers. However, a subsequent survey of $\mathrm{FEV}_{1}$ in 303 of the original 629 borax workers, 7 years after the original survey, found no exposure-related changes in $\mathrm{FEV}_{1}$ over this period, when adjustments were made for the effects of age, height, and smoking on $\mathrm{FEV}_{1}$ (Wegman et al. 1994). Acute-duration laboratory exposures of volunteers to sodium borate dust support the findings of respiratory irritation reported in the occupational studies.

The occurrence of acute respiratory symptoms as a possible consequence of acute exposure to dusts of sodium borate (the decahydrate, pentahydrate, and anhydrous tetraborates) was studied in a later study by interviewing workers about acute irritation symptoms before the work shift began and at regular hourly intervals during the work shift, and by measuring personal air concentrations of particulates at concurrent intervals for 4 consecutive days (Hu et al. 1992; Wegman et al. 1991, 1994). Seventy-nine exposed production workers and 27 nonexposed workers were included in the study. In the latest analysis of the collected data, the incidence rates for irritation symptoms in exposed workers were statistically significantly higher than those in nonexposed workers, with exposed workers 9-, 5-, and 3-fold more likely to report incidents of nasal, eye, and throat irritation, respectively, than comparison workers (Hu et al. 1992; Wegman et al. 1991, 1994). For the unexposed groups, the arithmetic means of the 6-hour TWA daily dust concentration was $0.45 \mathrm{mg}$ particulates $/ \mathrm{m}^{3}\left(0.02 \mathrm{mg}\right.$ boron $\left./ \mathrm{m}^{3}\right)$, with $100 \%$ of samples $\leq 1.0 \mathrm{mg}$ particulates $/ \mathrm{m}^{3}$ and about $90 \%$ of samples $\leq 0.5 \mathrm{mg}$ particulates $/ \mathrm{m}^{3}$. The arithmetic mean of the 6-hour TWA daily dust concentrations for the exposed group was $5.72 \mathrm{mg}$ particulates $/ \mathrm{m}^{3}(0.44 \mathrm{mg}$ boron $/ \mathrm{m}^{3}$ ), with a majority of air concentrations between 1.0 and $10.0 \mathrm{mg}$ particulates $/ \mathrm{m}^{3}$ (Wegman et al. 1991, 1994). Several factors make it difficult to identify a NOAEL or LOAEL for this study, precluding inclusion of this study in Figure and Table 3-1. Study participants rated their perception of individual episodes of eye, throat, and nasal irritancy on a scale of 0 (not at all) to 10 (very, very much), with ratings of 1, 2, and 3 representing "very little", "fairly little", and "moderate" irritation, respectively. Unexposed responders to the pre-shift survey reported a mean rating of 1.9 for all symptoms, while the mean rating for nasal irritation (the most commonly reported effect) among exposed workers was just slightly higher at 2.2. Thus, the cutoff for boron dust-induced effects is likely to be for ratings of $\geq 3$. In the exposed 
group, $91 \%$ of reported symptoms were rated with severity scores $\leq 3$ and $96 \%$ of symptoms were rated with severity scores of $\leq 4$ ("pretty much"). For incidences in which the workers depressed a counter button to record time of symptom onset, and the study surveyor recorded an occurrence of effect, the probability of reporting an effect did not change markedly until 0.25-hour TWA exposure levels reached 0.8-1.2 $\mathrm{mg}$ boron $/ \mathrm{m}^{3}$ (Wegman et al. 1991). There was no significant difference in reporting of symptoms based on the type of borate dust, which differ in boron content by almost a factor of two (i.e., $4.7 \mathrm{mg}$ anhydrous borax $/ \mathrm{m}^{3}$ and $8.8 \mathrm{mg} / \mathrm{m}^{3}$ of the decahydrate provide $1 \mathrm{mg}$ boron $/ \mathrm{m}^{3}$ ). Finally, the data do not indicate a temporal increase in effect intensity, as would be expected for a local irritant.

An uncertainty factor of 3 for human variability was based on the fact that inhaled borates and boric acid exert their adverse effects on respiratory tract tissues as portal-of-entry irritants. For portal-of-entry inhalation toxicants, the variability between humans in pharmacokinetics (i.e., toxicant deposition in the respiratory tract) is minimal. Thus, half $\left(10^{0.5}\right.$, or 3$)$ of the composite uncertainty factor for pharmacokinetic and pharmacodynamic variability in humans should be applied to the identified NOAEL. 


\title{
MINIMAL RISK LEVEL (MRL) WORKSHEET
}

\author{
Chemical Name: \\ Boron and Compounds \\ CAS Number: \\ 7440-42-8 \\ Date: \\ August 2009 \\ Profile Status: \\ Final Draft Post-Public Comment \\ Route: \\ Duration: \\ Graph Key: \\ Species: \\ [ ] Inhalation [X] Oral \\ [X] Acute [ ] Intermediate [ ] Chronic \\ 22 \\ Rabbit \\ Minimal Risk Level: 0.2 [X] mg/kg/day [ ] ppm
}

Reference: Price CJ, Marr MC, Myers CB, et al. 1996b. The developmental toxicity of boric acid in rabbits. Fundam Appl Toxicol 34:176-187.

The results of this study have also been reported in the following references:

Heindel JJ, Price CJ, Schwetz BA. 1994. The developmental toxicity of boric acid in mice, rats, and rabbits. Environ Health Perspect Suppl 102(7):107-112.

NTP. 1991. Final report on the developmental toxicity of boric acid (CAS No. 10043-35-3) in New Zealand white rabbits. Research Triangle Park, NC: U.S. Department of Health and Human Services, Public Health Service, National Toxicology Program. PB92129550.

Experimental design: Groups of 30 pregnant New Zealand white rabbits were given gavage doses of 0 , $62.5,125$, or $250 \mathrm{mg}$ boric acid/kg/day $(0,11,22$, or $44 \mathrm{mg}$ boron/kg/day) on gestation days 6-19. Observations were made for clinical signs, maternal and fetal body weight, number of implantations, resorptions, number of live and dead fetuses, and fetal external, visceral, and skeletal defects.

Effects noted in study and corresponding doses: No adverse maternal effects were observed in the 11 or $22 \mathrm{mg}$ boron/kg/day groups. At $44 \mathrm{mg}$ boron/kg/day, decreases in maternal body weight, relative kidney weight, and food consumption were observed. During the treatment period, the rabbits lost $137 \mathrm{~g}$ body weight compared to a weight gain of $93 \mathrm{~g}$ in controls. No differences in the number of implantation sites per litter were observed; however, there were significant increases in the percent resorptions per litter (6.3, 5.9, 7.7, and $89.9 \%$ in the $0,11,22$, and $44 \mathrm{mg}$ boron/ $\mathrm{kg} /$ day groups, respectively), percent of litters with one or more resorptions (39, 39, 45, and 95\%), and percent of litters with $100 \%$ resorption (0, 0, 0 , and $73 \%$ ). The number of live litters was 18, 23, 20, and 6 in the $0,11,22$, and $44 \mathrm{mg}$ boron $/ \mathrm{kg} / \mathrm{day}$ groups, respectively, and the number of live fetuses was 159, 175, 153, and 14, respectively. A decrease in fetal body weights (92\% of controls) was observed at $44 \mathrm{mg}$ boron $/ \mathrm{kg} / \mathrm{day}$; although the body weight was not significantly different from controls, the effect was considered biologically significant.

Significant increases in the percent of fetuses per litter with external $(0.8,1.4,1.0$, and $11.1 \%$ in the 0,11 , 22, and $44 \mathrm{mg}$ boron/kg/day groups, respectively), visceral (7.3, 5.9, 7.4, and 80.6\%), cardiovascular malformations (2.7, 3.1, 4.2, and 72.2\%) and cardiovascular variations (10.6, 5.7, 7.2, and 63.9\%) were observed. Although the overall incidence of external malformations was increased at $44 \mathrm{mg}$ boron/kg/day, there were no increases in a specific malformation. The visceral malformations primarily consisted of cardiovascular malformations, particularly interventricular septal defect, enlarged aorta, papillary muscle malformation, and double outlet right ventricle. The cardiovascular variations consisted of abnormal number of cardiac papillary muscles. 
Dose and end point used for MRL derivation: NOAEL of $22 \mathrm{mg}$ boron/kg/day as boric acid associated with a LOAEL of $44 \mathrm{mg}$ boron/kg/day as boric acid for developmental effects

\section{[X] NOAEL [ ] LOAEL}

\section{Uncertainty Factors used in MRL derivation:}

[ ] 10 for use of a LOAEL

[X] 10 for extrapolation from animals to humans

[X] 10 for human variability

Was a conversion used from ppm in food or water to a mg/body weight dose? No.

If an inhalation study in animals, list the conversion factors used in determining human equivalent dose: Not applicable.

Other additional studies or pertinent information that lend support to this MRL: A series of studies conducted by Cherrington and Chernoff (2002) also examined the developmental toxicity of boron. A variety of skeletal malformations (including rib agenesis, cervical rib, and fused ribs) were observed in the fetuses of mice receiving two gavage doses of $70 \mathrm{mg}$ boron/kg on gestation day 8 or gestation days 68 , once daily dose of $88 \mathrm{mg}$ boron/ $\mathrm{kg} /$ day on gestation days $6-10$, or one dose of $131 \mathrm{mg}$ boron $/ \mathrm{kg}$ on gestation day 8. Multiple thoracic skeletal malformations were observed in the fetuses of mice receiving two doses of $131 \mathrm{mg}$ boron/kg on gestation day 8. Decreases in fetal body weight were also observed in these studies and in studies of mice receiving two gavage doses of $70 \mathrm{mg}$ boron/kg on gestation day 6, 7, 9, or 10 .

Developmental effects have also been observed in intermediate-duration studies. Decreases in fetal body weight were observed in rats exposed to 13 or $13.6 \mathrm{mg}$ boron/ $\mathrm{kg} /$ day on gestation days 0-20 (Heindel et al. 1992; Price et al. 1996a), increases in skeletal abnormalities were observed in rats exposed to $13 \mathrm{mg}$ boron $/ \mathrm{kg} /$ day on gestation days 0-20 (Price et al. 1996a), and rib cage defects and enlargement of the brain lateral ventricles were observed in rats exposed to $28.4 \mathrm{mg}$ boron/ $\mathrm{kg} /$ day on gestation days 0 20 (Heindel et al. 1992). In mice exposed to boric acid on gestation days $0-17$, reduced fetal body weight and increased skeletal defects were observed at 79 and $175.3 \mathrm{mg}$ boron/kg/day, respectively. 


\section{MINIMAL RISK LEVEL (MRL) WORKSHEET}

$\begin{array}{ll}\text { Chemical Name: } & \text { Boron and Compounds } \\ \text { CAS Number: } & 7440-42-8 \\ \text { Date: } & \text { August 2009 } \\ \text { Profile Status: } & \text { Final Draft Post-Public Comment } \\ \text { Route: } & \text { [ ] Inhalation [X] Oral } \\ \text { Duration: } & \text { [ ] Acute [X] Intermediate [ ] Chronic } \\ \text { Graph Key: } & 60,61 \\ \text { Species: } & \text { Rat } \\ & \\ \text { Minimal Risk Level: } & 0.2[\mathrm{X}] \mathrm{mg} / \mathrm{kg} / \text { day [ ] ppm }\end{array}$

Reference: Heindel JJ, Price CJ, Field EA, et al. 1992. Developmental toxicity of boric acid in mice and rats. Fundam Appl Toxicol 18:266-277.

Experimental design: Groups of 26-28 pregnant Sprague-Dawley rats and Swiss mice were exposed to 0, $0.1,0.2$, or $0.4 \%$ boric acid in the diet on gestation days $0-20$. Estimated boron doses are $0,13.6$, 28.5, or $57.7 \mathrm{mg}$ boron/ $\mathrm{kg} /$ day $(0,78,163$, or $330 \mathrm{mg}$ boric acid/kg/day) for rats and 0,43 , 79, or $176 \mathrm{mg}$ boron $/ \mathrm{kg} /$ day (0, 248, 452, or 1,003 mg boric acid/kg/day) for mice. Daily observations were made for clinical signs and food and water consumption. At death, body and organ weights were recorded. Maternal kidneys were examined microscopically. Live fetuses were excised, anesthetized, weighed, and examined for skeletal malformations.

Effects noted in study and corresponding doses: Decreased maternal weight gain was observed in the $57.7 \mathrm{mg}$ boron $/ \mathrm{kg} /$ day group of rats, but not when corrected for gravid uterine weight. Decreased relative kidney and liver weights were seen in the $28.4 \mathrm{mg}$ boron/kg/day group. The incidence and severity of the minimal maternal nephropathy was not dose-related. Mean fetal body weight per litter was significantly reduced (7-15\%) in all treated groups. Significant increases in the percentage of malformed fetuses/litter or litter with one or more malformed fetuses was observed at doses $\geq 28.5 \mathrm{mg}$ boron $/ \mathrm{kg} / \mathrm{day}$. Noted malformations included anomalies of the eye, central nervous system, cardiovascular system, and axial skeleton. Enlarged lateral ventricles of the brain and agenesis or shortening of the $13^{\text {th }}$ rib were seen in the $57.7 \mathrm{mg}$ boron/kg/day group.

Reference: Price PJ, Strong PL, Marr MC, et al. 1996a. Developmental toxicity NOAEL and postnatal recovery in rats fed boric acid during gestation. Fundam Appl Toxicol 32:179-193.

Experimental design: Groups of 60 female Sprague-Dawley rats were exposed to 0, 0.025, 0.050, 0.075, 0.100 , or $0.200 \%$ boric acid in the diet on gestation days $0-20$. Observations were made for body weight, clinical signs, and food and water consumption. The study was performed in two phases; offspring were evaluated in both phases for post-implantation mortality, body weight, and external, visceral, and skeletal morphology. Phase I was terminated on gestation day 20. The calculated average maternal dose of boron was $0,3.3,6.3,10,13$, or $25 \mathrm{mg}$ boron $/ \mathrm{kg} /$ day $(0,19,36,55$, 76, or $143 \mathrm{mg}$ boric acid/ $/ \mathrm{kg} / \mathrm{day})$. Phase II dams were allowed to litter and rear their pups until postnatal day (pnd) 21 . For these dams, the calculated average doses of boron were $0,0.2,6.5,9.7,12.9$, and $25.3 \mathrm{mg} / \mathrm{kg} / \mathrm{day}(0,19,37,56,74$, and $145 \mathrm{mg}$ boric acid $/ \mathrm{kg} /$ day). During this phase, the incidence of skeletal defects in control and exposed pups was evaluated at the end of the first 21 postnatal days.

Effects noted in study and corresponding doses: During Phase I of the study, no maternal deaths or clinical signs were associated with boric acid treatment. When corrected for gravid uterine weight, 
maternal weight gain was not affected. However, reduced gravid uterine weight resulted in significant trend tests for decreased maternal body weight (gestation days19 and 20) and decreased maternal body weight gain (gestation days 15-18 and 0-20). Dams in the $25 \mathrm{mg}$ boron/kg/day group had a 10\% reduction (statistically significant in the trend test, $\mathrm{p}<0.05$ ) in gravid uterine weight compared with controls. Fetal body weights were significantly decreased in the 13 and $25 \mathrm{mg}$ boron/kg/day groups (6 and 12\% less than controls) on gestation day 20. Incidences of external or visceral malformations or variations were not treatment-related. However, a significant increase was observed for percentage of fetuses with skeletal malformations (short rib XIII) per litter and variations (wavy rib or wavy rib cartilage) in the 13 and $25 \mathrm{mg}$ boron/kg/day groups. A significant trend test $(\mathrm{p}<0.05)$ resulted for decrease in rudimentary extra rib on lumbar I (a variation). The LOAEL for Phase I of this study was identified as $13 \mathrm{mg}$ boron/kg/day, based on decreased fetal body weight and skeletal malformations. The NOAEL for this phase was identified as $10 \mathrm{mg}$ boron/kg/day.

In the Phase II study, a significant trend for increased number and percent of dead pups was seen between pnd 0 and 4, but not between pnd 4 and 21. This appeared to be due to the non-significant early postnatal mortality in the $25.3 \mathrm{mg}$ boron/ $\mathrm{kg} /$ day group. There were no effects of boric acid on the pup body weight from pnd 0 to 21; therefore, fetal body weight deficits (identified in Phase I) did not continue into the postnatal period (Phase II). The percentage of pups per litter with short rib XIII was increased on pnd $21 \mathrm{in} \mathrm{the} 25.3 \mathrm{mg}$ boron $/ \mathrm{kg} /$ day group. A LOAEL of $25.3 \mathrm{mg}$ boron $/ \mathrm{kg} / \mathrm{day}$, with an associated NOAEL of $12.9 \mathrm{mg}$ boron/kg/day, was identified for skeletal malformations in Phase II of this study.

Dose and end point used for MRL derivation: BMDL $_{05}$ of $10.3 \mathrm{mg} / \mathrm{kg} / \mathrm{day}$ for reduced fetal body weight

\section{[ ] NOAEL [ ] LOAEL [X] BMDL 05}

Allen et al. (1996) performed multiple benchmark dose (BMD) analyses on single-study or combined data from Heindel et al. (1992) and Price et al. (1996a) for all statistically significant developmental end points (Table A-1). Fetal body weight changes were analyzed using the average fetal weight for each litter with live fetuses. The modeling of rib effects aimed to differentiate whether treatment-related differences in the lumbar rib were variations or malformations. Thus, a weighting scheme was applied to represent three possible interpretations of severity of this effect; that is, a missing rib is: (a) trivially different from "normal" (1/6 weighting), (b) intermediate between a trivial or frank malformation (1/2 weighting), or (c) considered a frank malformation (5/6 weighting). Rib count analysis involved adjusting up (if rudimentary or full lumbar ribs present) or down (shortened rib XIII or rib agenesis) the base count of 13 rib pairs for each fetus analyzed. Benchmark responses (BMRs) were chosen for each end point. The BMD expected to result in the BMR, while the $\mathrm{BMDL}_{05}$ was defined as the $95 \%$ lower bound on the BMD. The data were modeled with a continuous power model using an F-test evaluation of goodness of fit. 


\section{Table A-1. Benchmark Dose Modeling of Developmental Effects of Oral Boric Acid Exposure to Rats}

\begin{tabular}{|c|c|c|c|c|}
\hline End point & Study data & $\begin{array}{l}\text { Goodness-of-fit } \\
\text { p-value }\end{array}$ & $\begin{array}{l}\text { BMD }^{\text {b }} \\
\text { (mg boron/ } \\
\text { kg/day) }\end{array}$ & $\begin{array}{l}\text { Lower bound } \\
\text { on BMD } \\
\text { (mg boron/ } \\
\text { kg/day) }\end{array}$ \\
\hline \multirow{3}{*}{$\begin{array}{l}\text { Fetal body weight as } \\
\text { continuous data } \\
\text { (BMR=5\% reduction) }\end{array}$} & Heindel et al. 1994 & 0.24 & 14.0 & 9.8 \\
\hline & Price et al. 1996a & 0.89 & 11.9 & 8.2 \\
\hline & Combined & 0.58 & 13.7 & 10.3 \\
\hline \multirow{3}{*}{$\begin{array}{l}\text { Fetal body weight as } \\
\text { continuous data } \\
\text { (BMR=1/2 standard deviation } \\
\text { below control) }\end{array}$} & Heindel et al. 1994 & 0.24 & 12.8 & 8.4 \\
\hline & Price et al. 1996a & 0.89 & 8.6 & 5.4 \\
\hline & Combined & 0.58 & 11.4 & 8.4 \\
\hline \multirow{3}{*}{$\begin{array}{l}\text { Fetal body weight as } \\
\text { dichotomous incidence data } \\
\text { (BMR }=5 \% \text { reduction) }\end{array}$} & Heindel et al. 1994 & 0.44 & 22.6 & 20.1 \\
\hline & Price et al. 1996a & 0.01 & 8.2 & 5.4 \\
\hline & Combined & NA & NA & NA \\
\hline \multirow{3}{*}{$\begin{array}{l}\text { Shortening or agenesis of rib } \\
\text { XIII }\end{array}$} & Heindel et al. 1994 & 0.07 & 24.9 & 18.6 \\
\hline & Price et al. 1996a & 0.64 & 29.9 & 21.5 \\
\hline & Combined & 0.42 & 24.5 & 21.0 \\
\hline \multirow[t]{3}{*}{ Missing lumbar ribs } & Heindel et al. 1994 & 0.99 & 1.2 & 0.3 \\
\hline & Price et al. 1996a & 0.78 & 1.5 & 0.6 \\
\hline & Combined & 0.99 & 2.1 & 0.9 \\
\hline \multirow{3}{*}{$\begin{array}{l}\text { Rib effects analysis: } \\
1 / 6 \text { weighting for absence of } \\
\text { lumbar rib }\end{array}$} & Heindel et al. 1994 & 0.27 & 21.2 & 16.5 \\
\hline & Price et al. 1996a & 0.78 & 32.9 & 25.7 \\
\hline & Combined & NA & NA & NA \\
\hline \multirow{3}{*}{$\begin{array}{l}\text { Rib effects analysis: } \\
1 / 2 \text { weighting for absence of } \\
\text { lumbar rib }\end{array}$} & Heindel et al. 1994 & 0.02 & 13.5 & 10.2 \\
\hline & Price et al. 1996a & 0.64 & 45.3 & 30.3 \\
\hline & Combined & NA & NA & NA \\
\hline \multirow{3}{*}{$\begin{array}{l}\text { Rib effects analysis: } \\
5 / 6 \text { weighting for absence of } \\
\text { lumbar rib }\end{array}$} & Heindel et al. 1994 & $<0.001$ & 24.9 & 20.5 \\
\hline & Price et al. 1996a & 0.53 & 53.7 & 31.2 \\
\hline & Combined & NA & NA & NA \\
\hline \multirow{3}{*}{$\begin{array}{l}\text { Rib effects analysis: } \\
\text { rib count for absence of } \\
\text { lumbar rib }\end{array}$} & Heindel et al. 1994 & 0.002 & 16.5 & 12.8 \\
\hline & Price et al. 1996a & 0.08 & 25.6 & 16.5 \\
\hline & Combined & NA & NA & NA \\
\hline
\end{tabular}

${ }^{a} p$-values for assessing adequacy of the models for predicting the observed data of Heindel et al. (1992) and Price et al. (1996a)

${ }^{\mathrm{b}}$ Benchmark dose: model estimated dose expected to result in the BMR

${ }^{\mathrm{c}} 95 \%$ lower bound on the BMD

$\mathrm{BMR}=$ benchmark response $; \mathrm{NA}=$ not applicable

Source: Heindel et al. 1992; Price et al. 1996a 
A likelihood ratio test indicated that the response data from both studies could be modeled as a single dose-response function. Of the developmental end points modeled, the lowest resulting BMDL 05 was $10.3 \mathrm{mg}$ boron/kg/day for fetal body weight (litter weight averages), which was similar to the NOAEL of $10 \mathrm{mg}$ boron/kg/day from the Price et al. (1996a) study.

Uncertainty Factors used in MRL derivation: A total uncertainty factor of 66 was used.

[ ] 10 for use of a LOAEL

[X] 3.3 for extrapolation of toxicokinetics from animals to humans

[X] 3.16 for extrapolation of toxicodynamics from animals to humans

[X] 2.0 for human toxicokinetic variability

[X] 3.16 for human toxicodynamic variability

In deriving a reference dose (RfD) for chronic oral exposures to boron, the U.S. EPA applied chemicalspecific uncertainty factors to the $\mathrm{BMDL}_{05}$ of $10.3 \mathrm{mg}$ boron $/ \mathrm{kg} /$ day reported by Allen et al. (1996) (EPA 2004). Rather than using the default uncertainty factors of 10 for interspecies extrapolation and 10 for interindividual human variability, each uncertainty factor was further delineated into toxicokinetic and toxicodynamic components specific to boron. Since the critical effect (reduced fetal body weight in animals) and point of departure (BMDL 05 of $10.3 \mathrm{mg} / \mathrm{kg} /$ day) for intermediate oral exposure to boron are the same as those for chronic oral exposures, as identified by EPA (2004), the chemical-specific uncertainty factors derived by U.S. EPA to derive a chronic RfD are appropriate for use in deriving the intermediate-duration MRL.

Briefly, each uncertainty factor of 10 for extrapolation from animals to humans and human variability was initially separated into default toxicokinetic and toxicodynamic adjustment factors of $3.16\left(10^{0.5}\right)$ each to account for species differences in toxicokinetic disposition and toxicodymanic responses to orallyingested boron. The same division was made for the uncertainty factor of 10 for human variability. Thus, the composite uncertainty factor $\left(\mathrm{UF}_{\mathrm{TOTAL}}\right)$ for the intermediate-duration oral $\mathrm{MRL}$ is defined as given by EPA (2004) as:

where:

$$
\mathrm{UF}_{\mathrm{TOTAL}}=\left(\mathrm{AF}_{\mathrm{AK}} \times \mathrm{AF}_{\mathrm{AD}} \times \mathrm{AF}_{\mathrm{HK}} \times \mathrm{AF}_{\mathrm{HD}} \times \mathrm{UF}\right)
$$

$$
\begin{aligned}
& A F_{A K}=\text { interspecies toxicokinetic adjustment factor } \\
& \mathrm{AF}_{\mathrm{AD}}=\text { interspecies toxicodynamic adjustment factor } \\
& \mathrm{AF}_{\mathrm{HK}}=\text { interindividual toxicokinetic adjustment factor } \\
& \mathrm{AF}_{\mathrm{HD}}=\text { interindividual toxicodynamic adjustment factor } \\
& \mathrm{UF}=\text { other uncertainty factors (e.g., use of a LOAEL instead of a NOAEL) }
\end{aligned}
$$

Since no data were available to adequately describe the mode(s) or mechanism(s) of action for boron toxicity in animals or humans, the default toxicodynamic adjustment factor of 3.16 was used to account for inter- and intraspecies uncertainties in toxicodynamics.

The pregnant female is considered to be a sensitive population for boron exposure, as fetal effects in rats are the most sensitive end point identified for boron toxicity. Since boron exhibits near first-order toxicokinetics, distributing freely between total body water and tissues (except for bone, in which it accumulates to approximately 4-fold that of plasma [Chapin et al. 1997]), variability between maternal and fetal kinetics should be essentially equal. Thus, maternal boron plasma concentration is an appropriate surrogate for fetal plasma levels. No data are available to relate rat and human plasma boron concentration. However, boron is not metabolized, but almost completely eliminated in the urine, making renal clearance an appropriate kinetic factor for comparison of toxicokinetic differences between rats and humans. Given the known distribution of boron to total body water and bone, two-compartment 
pharmacokinetic models for boron in rats and humans can describe plasma concentration in terms of renal clearance. Boron's toxicity is likely to be related to a continuous exposure over an extended portion of fetal development in which a steady state of circulating boron is achieved. Under the assumption of steady-state plasma boron levels, and assuming approximately complete clearance of born to urine, the two-compartment model can be simplified to the following expression:

where:

$$
C_{S S}=\left(D_{e} \times f_{a} \times B W\right) / C l
$$

$\mathrm{D}_{\mathrm{e}}=$ external dose of ingested boron (mg boron/kg body weight/day)

$\mathrm{f}_{\mathrm{a}}=$ fraction of ingested boron absorbed from the gut

$\mathrm{BW}=$ body weight $(\mathrm{kg})$

$\mathrm{Cl}=$ renal clearance $(\mathrm{mL} /$ minute $)$

Assuming that the ratio of 1 for internal, steady-state doses in rats and humans results in equivalent responses, the expressions for the plasma boron concentration in rats and humans can be expressed as the following ratio, which serves as the $\mathrm{AF}_{\mathrm{AK}}$ :

$$
\mathrm{AF}_{\mathrm{AK}}=\left(\mathrm{Cl}_{\mathrm{R}} \times \mathrm{f}_{\mathrm{AH}} \times \mathrm{BW}_{\mathrm{H}}\right) /\left(\mathrm{Cl}_{\mathrm{H}} \times \mathrm{f}_{\mathrm{AR}} \times \mathrm{BW}_{\mathrm{R}}\right)
$$

where the subscripts $\mathrm{R}$ and $\mathrm{H}$ represent rats and humans. Values for mean renal clearance of 1.0 and $66.1 \mathrm{~mL} /$ minute in pregnant rats and humans, respectively, were derived from the studies of Vaziri et al. (2001), and Pahl et al. (2001), which also provided pregnant rat and human body weights of 0.303 and $67.6 \mathrm{~kg}$, respectively. Using gastrointestinal absorption fractions of 0.92 (Schou et al. 1984) and 0.95 (Vanderpool et al. 1994) for $\mathrm{f}_{\mathrm{AH}}$ and $\mathrm{f}_{\mathrm{AR}}$, respectively, $\mathrm{AF}_{\mathrm{AK}}$ is derived as follows:

$$
\begin{aligned}
\mathrm{AF}_{\mathrm{AK}} & =(1.00 \times 0.92 \times 67.6) /(66.1 \times 0.95 \times 0.303) \\
& =62.2 / 19.0 \\
& =3.3
\end{aligned}
$$

The assessment of human variability in boron toxicokinetics utilized glomerular filtration rate (GFR) as a surrogate for renal clearance. Pregnant women were considered the sensitive population, particularly those women with compromised renal function (3-5\% preeclamptic women in the U.S. population). Using a modification of Dourson et al. (1998), data from women with normal renal function were used to define an $\mathrm{AF}_{\mathrm{HK}}$ as:

$$
\mathrm{AF}_{\mathrm{HK}}=\mathrm{GFR}_{\mathrm{AVG}} /\left(\mathrm{GFR}_{\mathrm{AVG}}-\left(3 \times \mathrm{SD}_{\mathrm{GFR}}\right)\right)
$$

where $\mathrm{GFR}_{\mathrm{AVG}}$ and $\mathrm{SD}_{\mathrm{GFR}}$ are mean and standard deviation of the GFR for healthy women. Three standard deviations below the mean GFR was chosen to account for the women with very low GFR. From the studies of Dunlop (1981), Krutzen et al. (1992), and Sturgiss et al. (1996), a mean GFR of $161.5 \mathrm{~mL} /$ minute and a mean GFR-3SD ${ }_{\mathrm{GFR}}$ of $85.8 \mathrm{~mL} /$ minute resulted in an $\mathrm{AF}_{\mathrm{HK}}$ of 1.93. This number was rounded to 2.0 to account for uncertainties in human GFR.

Based on these analyses, the total uncertainty factor applied to the $\mathrm{BMDL}_{05}$ of $10.3 \mathrm{mg}$ boron $/ \mathrm{kg}$ is derived as:

$$
\begin{aligned}
\mathrm{UF}_{\mathrm{TOTAL}} & =\left(\mathrm{AF}_{\mathrm{AK}} \times \mathrm{AF}_{\mathrm{AD}} \times \mathrm{AF}_{\mathrm{HK}} \times \mathrm{AF}_{\mathrm{HD}} \times \mathrm{UF}\right) \\
& =(3.3 \times 3.16 \times 2.0 \times 3.16 \times 1) \\
& =66
\end{aligned}
$$

Was a conversion used from ppm in food or water to a mg/body weight dose? No. 
If an inhalation study in animals, list the conversion factors used in determining human equivalent dose: Not applicable.

Other additional studies or pertinent information that lend support to this MRL: Reproductive effects, including testicular atrophy and histopathology, sperm abnormalities, and reduced sperm production have been observed in mice, rats, and dogs after intermediate-duration ingestion of doses of $26 \mathrm{mg}$ boron/kg/day (as boric acid or borax) and higher (Dixon et al. 1979; Fukuda et al. 2000; Harris et al. 1992; Ku et al. 1993a; Kudo et al. 2000; Seal and Weeth 1980; Treinen and Chapin 1991; Weir and Fisher 1972; Yoshizaki et al. 1999). Systemic effects have been observed in rats and dogs at higher doses. Hematological alterations (splenic extramedullary hematopoiesis and decreased hemoglobin levels) have been observed at 60.5 or $72 \mathrm{mg}$ boron/kg/day (NTP 1987; Weir and Fisher 1972), desquamation of skin on paws and tail and inflamed eyes have been observed in rats exposed to $150 \mathrm{mg}$ boron/kg/day (Weir and Fisher 1972), and hyperkeratosis and/or acanthosis of the stomach has been observed at $577 \mathrm{mg}$ boron/kg/day (NTP 1987).

Agency Contacts (Chemical Managers): Malcolm Williams 
APPENDIX A

This page is intentionally blank. 


\section{APPENDIX B. USER'S GUIDE}

\section{Chapter 1}

\section{Public Health Statement}

This chapter of the profile is a health effects summary written in non-technical language. Its intended audience is the general public, especially people living in the vicinity of a hazardous waste site or chemical release. If the Public Health Statement were removed from the rest of the document, it would still communicate to the lay public essential information about the chemical.

The major headings in the Public Health Statement are useful to find specific topics of concern. The topics are written in a question and answer format. The answer to each question includes a sentence that will direct the reader to chapters in the profile that will provide more information on the given topic.

\section{Chapter 2}

\section{Relevance to Public Health}

This chapter provides a health effects summary based on evaluations of existing toxicologic, epidemiologic, and toxicokinetic information. This summary is designed to present interpretive, weightof-evidence discussions for human health end points by addressing the following questions:

1. What effects are known to occur in humans?

2. What effects observed in animals are likely to be of concern to humans?

3. What exposure conditions are likely to be of concern to humans, especially around hazardous waste sites?

The chapter covers end points in the same order that they appear within the Discussion of Health Effects by Route of Exposure section, by route (inhalation, oral, and dermal) and within route by effect. Human data are presented first, then animal data. Both are organized by duration (acute, intermediate, chronic). In vitro data and data from parenteral routes (intramuscular, intravenous, subcutaneous, etc.) are also considered in this chapter.

The carcinogenic potential of the profiled substance is qualitatively evaluated, when appropriate, using existing toxicokinetic, genotoxic, and carcinogenic data. ATSDR does not currently assess cancer potency or perform cancer risk assessments. Minimal Risk Levels (MRLs) for noncancer end points (if derived) and the end points from which they were derived are indicated and discussed.

Limitations to existing scientific literature that prevent a satisfactory evaluation of the relevance to public health are identified in the Chapter 3 Data Needs section.

\section{Interpretation of Minimal Risk Levels}

Where sufficient toxicologic information is available, ATSDR has derived MRLs for inhalation and oral routes of entry at each duration of exposure (acute, intermediate, and chronic). These MRLs are not meant to support regulatory action, but to acquaint health professionals with exposure levels at which adverse health effects are not expected to occur in humans. 
MRLs should help physicians and public health officials determine the safety of a community living near a chemical emission, given the concentration of a contaminant in air or the estimated daily dose in water. MRLs are based largely on toxicological studies in animals and on reports of human occupational exposure.

MRL users should be familiar with the toxicologic information on which the number is based. Chapter 2, "Relevance to Public Health," contains basic information known about the substance. Other sections such as Chapter 3 Section 3.9, "Interactions with Other Substances," and Section 3.10, "Populations that are Unusually Susceptible" provide important supplemental information.

MRL users should also understand the MRL derivation methodology. MRLs are derived using a modified version of the risk assessment methodology that the Environmental Protection Agency (EPA) provides (Barnes and Dourson 1988) to determine reference doses (RfDs) for lifetime exposure.

To derive an MRL, ATSDR generally selects the most sensitive end point which, in its best judgement, represents the most sensitive human health effect for a given exposure route and duration. ATSDR cannot make this judgement or derive an MRL unless information (quantitative or qualitative) is available for all potential systemic, neurological, and developmental effects. If this information and reliable quantitative data on the chosen end point are available, ATSDR derives an MRL using the most sensitive species (when information from multiple species is available) with the highest no-observed-adverse-effect level (NOAEL) that does not exceed any adverse effect levels. When a NOAEL is not available, a lowest-observed-adverse-effect level (LOAEL) can be used to derive an MRL, and an uncertainty factor (UF) of 10 must be employed. Additional uncertainty factors of 10 must be used both for human variability to protect sensitive subpopulations (people who are most susceptible to the health effects caused by the substance) and for interspecies variability (extrapolation from animals to humans). In deriving an MRL, these individual uncertainty factors are multiplied together. The product is then divided into the inhalation concentration or oral dosage selected from the study. Uncertainty factors used in developing a substance-specific MRL are provided in the footnotes of the levels of significant exposure (LSE) tables.

\section{Chapter 3}

\section{Health Effects}

\section{Tables and Figures for Levels of Significant Exposure (LSE)}

Tables and figures are used to summarize health effects and illustrate graphically levels of exposure associated with those effects. These levels cover health effects observed at increasing dose concentrations and durations, differences in response by species, MRLs to humans for noncancer end points, and EPA's estimated range associated with an upper- bound individual lifetime cancer risk of 1 in 10,000 to 1 in 10,000,000. Use the LSE tables and figures for a quick review of the health effects and to locate data for a specific exposure scenario. The LSE tables and figures should always be used in conjunction with the text. All entries in these tables and figures represent studies that provide reliable, quantitative estimates of NOAELs, LOAELs, or Cancer Effect Levels (CELs).

The legends presented below demonstrate the application of these tables and figures. Representative examples of LSE Table 3-1 and Figure 3-1 are shown. The numbers in the left column of the legends correspond to the numbers in the example table and figure. 


\section{LEGEND}

\section{See Sample LSE Table 3-1 (page B-6)}

(1) Route of Exposure. One of the first considerations when reviewing the toxicity of a substance using these tables and figures should be the relevant and appropriate route of exposure. Typically when sufficient data exist, three LSE tables and two LSE figures are presented in the document. The three LSE tables present data on the three principal routes of exposure, i.e., inhalation, oral, and dermal (LSE Tables 3-1, 3-2, and 3-3, respectively). LSE figures are limited to the inhalation (LSE Figure 3-1) and oral (LSE Figure 3-2) routes. Not all substances will have data on each route of exposure and will not, therefore, have all five of the tables and figures.

(2) $\quad$ Exposure Period. Three exposure periods-acute (less than 15 days), intermediate (15364 days), and chronic (365 days or more)—are presented within each relevant route of exposure. In this example, an inhalation study of intermediate exposure duration is reported. For quick reference to health effects occurring from a known length of exposure, locate the applicable exposure period within the LSE table and figure.

(3) Health Effect. The major categories of health effects included in LSE tables and figures are death, systemic, immunological, neurological, developmental, reproductive, and cancer. NOAELs and LOAELs can be reported in the tables and figures for all effects but cancer. Systemic effects are further defined in the "System" column of the LSE table (see key number 18).

(4) Key to Figure. Each key number in the LSE table links study information to one or more data points using the same key number in the corresponding LSE figure. In this example, the study represented by key number 18 has been used to derive a NOAEL and a Less Serious LOAEL (also see the two "18r" data points in sample Figure 3-1).

(5) Species. The test species, whether animal or human, are identified in this column. Chapter 2, "Relevance to Public Health," covers the relevance of animal data to human toxicity and Section 3.4, "Toxicokinetics," contains any available information on comparative toxicokinetics. Although NOAELs and LOAELs are species specific, the levels are extrapolated to equivalent human doses to derive an MRL.

(6) Exposure Frequency/Duration. The duration of the study and the weekly and daily exposure regimens are provided in this column. This permits comparison of NOAELs and LOAELs from different studies. In this case (key number 18), rats were exposed to "Chemical x" via inhalation for 6 hours/day, 5 days/week, for 13 weeks. For a more complete review of the dosing regimen, refer to the appropriate sections of the text or the original reference paper (i.e., Nitschke et al. 1981).

(7) System. This column further defines the systemic effects. These systems include respiratory, cardiovascular, gastrointestinal, hematological, musculoskeletal, hepatic, renal, and dermal/ocular. "Other" refers to any systemic effect (e.g., a decrease in body weight) not covered in these systems. In the example of key number 18, one systemic effect (respiratory) was investigated.

(8) NOAEL. A NOAEL is the highest exposure level at which no harmful effects were seen in the organ system studied. Key number 18 reports a NOAEL of 3 ppm for the respiratory system, which was used to derive an intermediate exposure, inhalation MRL of 0.005 ppm (see footnote "b"). 
(9) LOAEL. A LOAEL is the lowest dose used in the study that caused a harmful health effect. LOAELs have been classified into "Less Serious" and "Serious" effects. These distinctions help readers identify the levels of exposure at which adverse health effects first appear and the gradation of effects with increasing dose. A brief description of the specific end point used to quantify the adverse effect accompanies the LOAEL. The respiratory effect reported in key number 18 (hyperplasia) is a Less Serious LOAEL of $10 \mathrm{ppm}$. MRLs are not derived from Serious LOAELs.

(10) Reference. The complete reference citation is given in Chapter 9 of the profile.

(11) CEL. A CEL is the lowest exposure level associated with the onset of carcinogenesis in experimental or epidemiologic studies. CELs are always considered serious effects. The LSE tables and figures do not contain NOAELs for cancer, but the text may report doses not causing measurable cancer increases.

(12) Footnotes. Explanations of abbreviations or reference notes for data in the LSE tables are found in the footnotes. Footnote "b" indicates that the NOAEL of 3 ppm in key number 18 was used to derive an MRL of 0.005 ppm.

\section{LEGEND}

\section{See Sample Figure 3-1 (page B-7)}

LSE figures graphically illustrate the data presented in the corresponding LSE tables. Figures help the reader quickly compare health effects according to exposure concentrations for particular exposure periods.

(13) Exposure Period. The same exposure periods appear as in the LSE table. In this example, health effects observed within the acute and intermediate exposure periods are illustrated.

(14) Health Effect. These are the categories of health effects for which reliable quantitative data exists. The same health effects appear in the LSE table.

(15) Levels of Exposure. Concentrations or doses for each health effect in the LSE tables are graphically displayed in the LSE figures. Exposure concentration or dose is measured on the log scale "y" axis. Inhalation exposure is reported in $\mathrm{mg} / \mathrm{m}^{3}$ or $\mathrm{ppm}$ and oral exposure is reported in $\mathrm{mg} / \mathrm{kg} / \mathrm{day}$.

(16) NOAEL. In this example, the open circle designated 18r identifies a NOAEL critical end point in the rat upon which an intermediate inhalation exposure MRL is based. The key number 18 corresponds to the entry in the LSE table. The dashed descending arrow indicates the extrapolation from the exposure level of $3 \mathrm{ppm}$ (see entry 18 in the table) to the MRL of 0.005 ppm (see footnote "b" in the LSE table).

(17) CEL. Key number $38 \mathrm{~m}$ is one of three studies for which CELs were derived. The diamond symbol refers to a CEL for the test species-mouse. The number 38 corresponds to the entry in the LSE table. 
(18) Estimated Upper-Bound Human Cancer Risk Levels. This is the range associated with the upperbound for lifetime cancer risk of 1 in 10,000 to 1 in 10,000,000. These risk levels are derived from the EPA's Human Health Assessment Group's upper-bound estimates of the slope of the cancer dose response curve at low dose levels $\left(\mathrm{q}_{1}{ }^{*}\right)$.

(19) Key to LSE Figure. The Key explains the abbreviations and symbols used in the figure. 
SAMPLE

Table 3-1. Levels of Significant Exposure to [Chemical x] - Inhalation

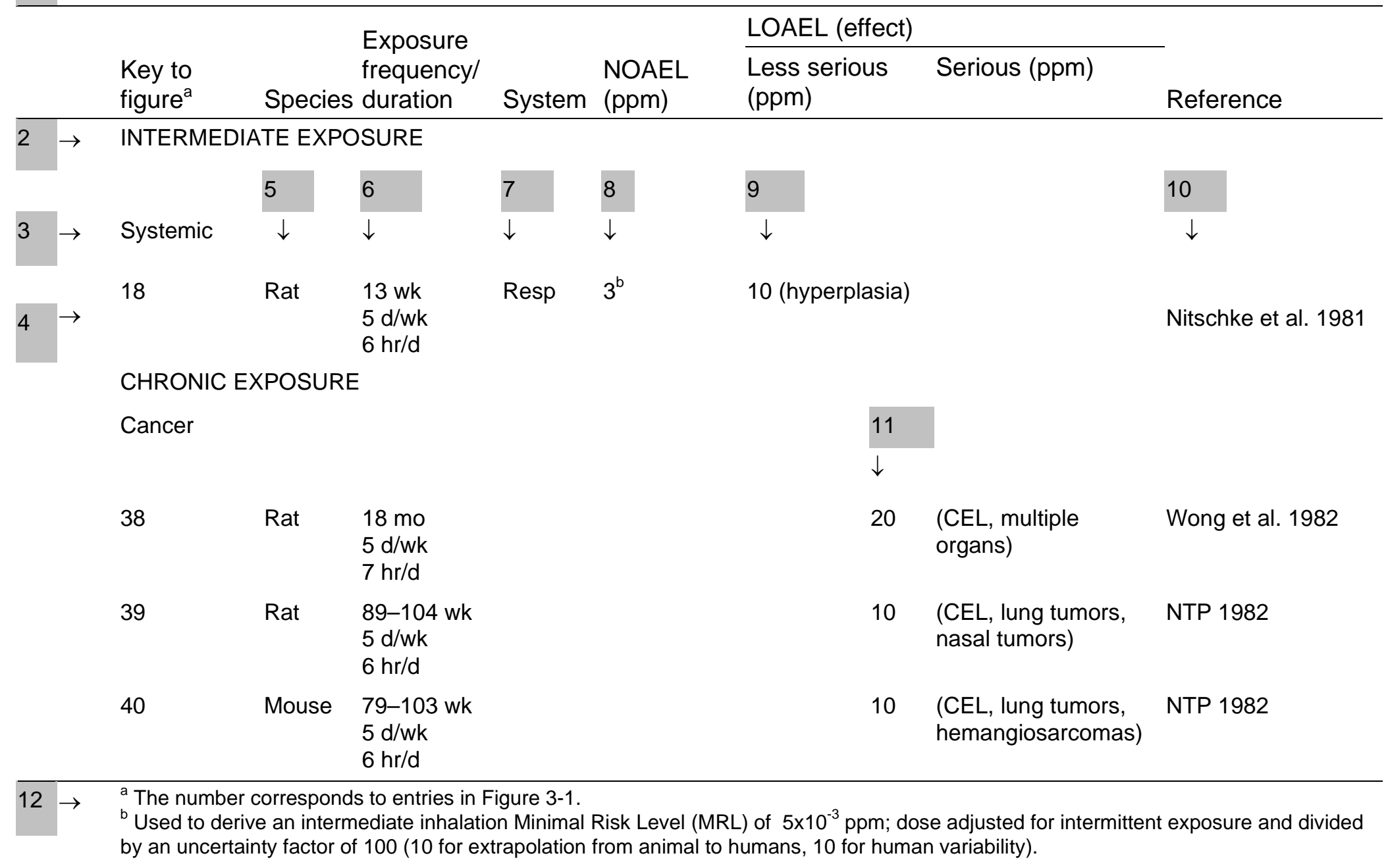




\section{SAMPLE}

13

Figure 3-1. Levels of Significant Exposure to [Chemical X] - Inhalation
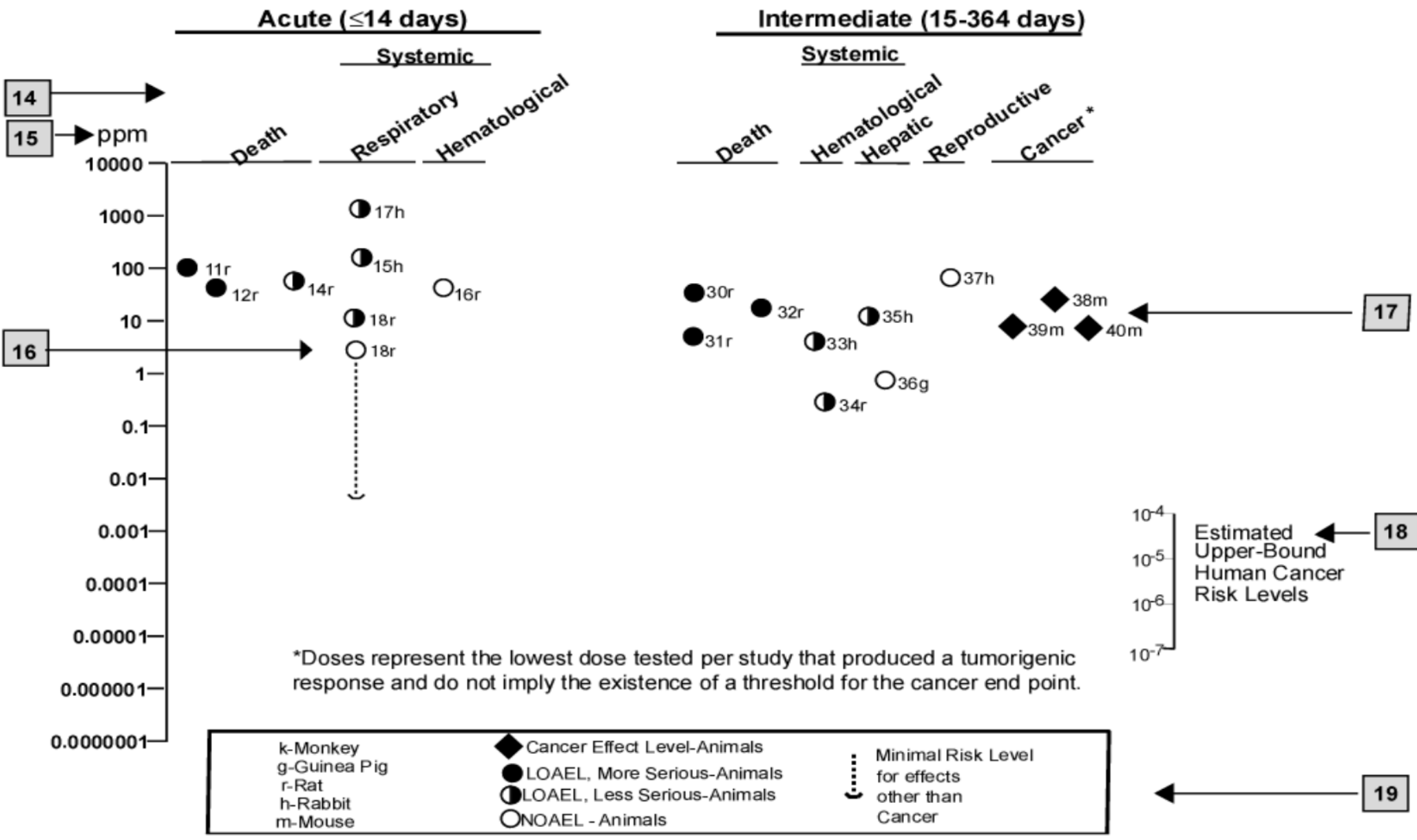
APPENDIX B

This page is intentionally blank. 


\section{APPENDIX C. ACRONYMS, ABBREVIATIONS, AND SYMBOLS}

\begin{tabular}{|c|c|}
\hline ACGIH & American Conference of Governmental Industrial Hygienists \\
\hline ACOEM & American College of Occupational and Environmental Medicine \\
\hline ADI & acceptable daily intake \\
\hline ADME & absorption, distribution, metabolism, and excretion \\
\hline AED & atomic emission detection \\
\hline AFID & alkali flame ionization detector \\
\hline AFOSH & Air Force Office of Safety and Health \\
\hline ALT & alanine aminotransferase \\
\hline AML & acute myeloid leukemia \\
\hline AOAC & Association of Official Analytical Chemists \\
\hline AOEC & Association of Occupational and Environmental Clinics \\
\hline $\mathrm{AP}$ & alkaline phosphatase \\
\hline APHA & American Public Health Association \\
\hline AST & aspartate aminotransferase \\
\hline atm & atmosphere \\
\hline ATSDR & Agency for Toxic Substances and Disease Registry \\
\hline AWQC & Ambient Water Quality Criteria \\
\hline BAT & best available technology \\
\hline BCF & bioconcentration factor \\
\hline BEI & Biological Exposure Index \\
\hline $\mathrm{BMD} / \mathrm{C}$ & benchmark dose or benchmark concentration \\
\hline $\mathrm{BMD}_{\mathrm{X}}$ & dose that produces a X\% change in response rate of an adverse effect \\
\hline $\mathrm{BMDL}_{X}$ & $95 \%$ lower confidence limit on the $\mathrm{BMD}_{\mathrm{X}}$ \\
\hline BMDS & Benchmark Dose Software \\
\hline BMR & benchmark response \\
\hline BSC & Board of Scientific Counselors \\
\hline $\mathrm{C}$ & centigrade \\
\hline CAA & Clean Air Act \\
\hline CAG & Cancer Assessment Group of the U.S. Environmental Protection Agency \\
\hline CAS & Chemical Abstract Services \\
\hline $\mathrm{CDC}$ & Centers for Disease Control and Prevention \\
\hline CEL & cancer effect level \\
\hline CELDS & Computer-Environmental Legislative Data System \\
\hline CERCLA & Comprehensive Environmental Response, Compensation, and Liability Act \\
\hline CFR & Code of Federal Regulations \\
\hline $\mathrm{Ci}$ & curie \\
\hline $\mathrm{CI}$ & confidence interval \\
\hline CL & ceiling limit value \\
\hline CLP & Contract Laboratory Program \\
\hline $\mathrm{cm}$ & centimeter \\
\hline CML & chronic myeloid leukemia \\
\hline CPSC & Consumer Products Safety Commission \\
\hline CWA & Clean Water Act \\
\hline DHEW & Department of Health, Education, and Welfare \\
\hline DHHS & Department of Health and Human Services \\
\hline DNA & deoxyribonucleic acid \\
\hline DOD & Department of Defense \\
\hline DOE & Department of Energy \\
\hline DOL & Department of Labor \\
\hline
\end{tabular}




\begin{tabular}{|c|c|}
\hline DOT & Department of Transportation \\
\hline DOT/UN/ & Department of Transportation/United Nations/ \\
\hline NA/IMDG & North America/Intergovernmental Maritime Dangerous Goods Code \\
\hline DWEL & drinking water exposure level \\
\hline ECD & electron capture detection \\
\hline ECG/EKG & electrocardiogram \\
\hline EEG & electroencephalogram \\
\hline EEGL & Emergency Exposure Guidance Level \\
\hline EPA & Environmental Protection Agency \\
\hline $\mathrm{F}$ & Fahrenheit \\
\hline $\mathrm{F}_{1}$ & first-filial generation \\
\hline FAO & Food and Agricultural Organization of the United Nations \\
\hline FDA & Food and Drug Administration \\
\hline FEMA & Federal Emergency Management Agency \\
\hline FIFRA & Federal Insecticide, Fungicide, and Rodenticide Act \\
\hline FPD & flame photometric detection \\
\hline fpm & feet per minute \\
\hline FR & Federal Register \\
\hline FSH & follicle stimulating hormone \\
\hline g & gram \\
\hline GC & gas chromatography \\
\hline gd & gestational day \\
\hline GLC & gas liquid chromatography \\
\hline GPC & gel permeation chromatography \\
\hline HPLC & high-performance liquid chromatography \\
\hline HRGC & high resolution gas chromatography \\
\hline HSDB & Hazardous Substance Data Bank \\
\hline IARC & International Agency for Research on Cancer \\
\hline IDLH & immediately dangerous to life and health \\
\hline ILO & International Labor Organization \\
\hline IRIS & Integrated Risk Information System \\
\hline $\mathrm{Kd}$ & adsorption ratio \\
\hline $\mathrm{kg}$ & kilogram \\
\hline kkg & metric ton \\
\hline $\mathrm{K}_{\mathrm{oc}}$ & organic carbon partition coefficient \\
\hline $\mathrm{K}_{\mathrm{ow}}$ & octanol-water partition coefficient \\
\hline $\mathrm{L}$ & liter \\
\hline $\mathrm{LC}$ & liquid chromatography \\
\hline $\mathrm{LC}_{50}$ & lethal concentration, $50 \%$ kill \\
\hline $\mathrm{LC}_{\mathrm{Lo}}$ & lethal concentration, low \\
\hline $\mathrm{LD}_{50}$ & lethal dose, 50\% kill \\
\hline $\mathrm{LD}_{\mathrm{Lo}}$ & lethal dose, low \\
\hline LDH & lactic dehydrogenase \\
\hline LH & luteinizing hormone \\
\hline LOAEL & lowest-observed-adverse-effect level \\
\hline LSE & Levels of Significant Exposure \\
\hline $\mathrm{LT}_{50}$ & lethal time, 50\% kill \\
\hline $\mathrm{m}$ & meter \\
\hline MA & trans,trans-muconic acid \\
\hline MAL & maximum allowable level \\
\hline $\mathrm{mCi}$ & millicurie \\
\hline
\end{tabular}




\begin{tabular}{|c|c|}
\hline MCL & maximum contaminant level \\
\hline MCLG & maximum contaminant level goal \\
\hline MF & modifying factor \\
\hline MFO & mixed function oxidase \\
\hline $\mathrm{mg}$ & milligram \\
\hline $\mathrm{mL}$ & milliliter \\
\hline $\mathrm{mm}$ & millimeter \\
\hline $\mathrm{mmHg}$ & millimeters of mercury \\
\hline mmol & millimole \\
\hline mppcf & millions of particles per cubic foot \\
\hline MRL & Minimal Risk Level \\
\hline MS & mass spectrometry \\
\hline NAAQS & National Ambient Air Quality Standard \\
\hline NAS & National Academy of Science \\
\hline NATICH & National Air Toxics Information Clearinghouse \\
\hline NATO & North Atlantic Treaty Organization \\
\hline NCE & normochromatic erythrocytes \\
\hline NCEH & National Center for Environmental Health \\
\hline NCI & National Cancer Institute \\
\hline ND & not detected \\
\hline NFPA & National Fire Protection Association \\
\hline ng & nanogram \\
\hline NHANES & National Health and Nutrition Examination Survey \\
\hline NIEHS & National Institute of Environmental Health Sciences \\
\hline NIOSH & National Institute for Occupational Safety and Health \\
\hline NIOSHTIC & NIOSH's Computerized Information Retrieval System \\
\hline NLM & National Library of Medicine \\
\hline $\mathrm{nm}$ & nanometer \\
\hline nmol & nanomole \\
\hline NOAEL & no-observed-adverse-effect level \\
\hline NOES & National Occupational Exposure Survey \\
\hline NOHS & National Occupational Hazard Survey \\
\hline NPD & nitrogen phosphorus detection \\
\hline NPDES & National Pollutant Discharge Elimination System \\
\hline NPL & National Priorities List \\
\hline NR & not reported \\
\hline NRC & National Research Council \\
\hline NS & not specified \\
\hline NSPS & New Source Performance Standards \\
\hline NTIS & National Technical Information Service \\
\hline NTP & National Toxicology Program \\
\hline ODW & Office of Drinking Water, EPA \\
\hline OERR & Office of Emergency and Remedial Response, EPA \\
\hline $\mathrm{OHM} / \mathrm{TADS}$ & Oil and Hazardous Materials/Technical Assistance Data System \\
\hline OPP & Office of Pesticide Programs, EPA \\
\hline OPPT & Office of Pollution Prevention and Toxics, EPA \\
\hline OPPTS & Office of Prevention, Pesticides and Toxic Substances, EPA \\
\hline OR & odds ratio \\
\hline OSHA & Occupational Safety and Health Administration \\
\hline OSW & Office of Solid Waste, EPA \\
\hline OTS & Office of Toxic Substances \\
\hline
\end{tabular}




\begin{tabular}{|c|c|}
\hline OW & Office of Water \\
\hline OWRS & Office of Water Regulations and Standards, EPA \\
\hline PAH & polycyclic aromatic hydrocarbon \\
\hline PBPD & physiologically based pharmacodynamic \\
\hline PBPK & physiologically based pharmacokinetic \\
\hline PCE & polychromatic erythrocytes \\
\hline PEL & permissible exposure limit \\
\hline pg & picogram \\
\hline PHS & Public Health Service \\
\hline PID & photo ionization detector \\
\hline pmol & picomole \\
\hline PMR & proportionate mortality ratio \\
\hline ppb & parts per billion \\
\hline ppm & parts per million \\
\hline ppt & parts per trillion \\
\hline PSNS & pretreatment standards for new sources \\
\hline RBC & red blood cell \\
\hline REL & recommended exposure level/limit \\
\hline RfC & reference concentration \\
\hline RfD & reference dose \\
\hline RNA & ribonucleic acid \\
\hline RQ & reportable quantity \\
\hline RTECS & Registry of Toxic Effects of Chemical Substances \\
\hline SARA & Superfund Amendments and Reauthorization Act \\
\hline SCE & sister chromatid exchange \\
\hline SGOT & serum glutamic oxaloacetic transaminase \\
\hline SGPT & serum glutamic pyruvic transaminase \\
\hline SIC & standard industrial classification \\
\hline SIM & selected ion monitoring \\
\hline SMCL & secondary maximum contaminant level \\
\hline SMR & standardized mortality ratio \\
\hline SNARL & suggested no adverse response level \\
\hline SPEGL & Short-Term Public Emergency Guidance Level \\
\hline STEL & short term exposure limit \\
\hline STORET & Storage and Retrieval \\
\hline $\mathrm{TD}_{50}$ & toxic dose, $50 \%$ specific toxic effect \\
\hline TLV & threshold limit value \\
\hline TOC & total organic carbon \\
\hline TPQ & threshold planning quantity \\
\hline TRI & Toxics Release Inventory \\
\hline TSCA & Toxic Substances Control Act \\
\hline TWA & time-weighted average \\
\hline UF & uncertainty factor \\
\hline U.S. & United States \\
\hline USDA & United States Department of Agriculture \\
\hline USGS & United States Geological Survey \\
\hline VOC & volatile organic compound \\
\hline WBC & white blood cell \\
\hline WHO & World Health Organization \\
\hline
\end{tabular}




$\begin{array}{ll}> & \text { greater than } \\ \geq & \text { greater than or equal to } \\ = & \text { equal to } \\ < & \text { less than } \\ \leq & \text { less than or equal to } \\ \% & \text { percent } \\ \alpha & \text { alpha } \\ \beta & \text { beta } \\ \gamma & \text { gamma } \\ \delta & \text { delta } \\ \mu \mathrm{m} & \text { micrometer } \\ \mu \mathrm{g} & \text { microgram } \\ \mathrm{q}_{1}{ }^{*} & \text { cancer slope factor } \\ - & \text { negative } \\ + & \text { positive } \\ (+) & \text { weakly positive result } \\ (-) & \text { weakly negative result }\end{array}$


APPENDIX C

This page is intentionally blank. 


\section{APPENDIX D. INDEX}

absorbed dose 101

adipose tissue

95,148

adrenals

$75,90,110$

adsorbed 140

adsorption.

134, 140, 141, 152

ambient air 142

bioaccumulation .153

bioconcentration factor 141

biomarker 100, 101, 115, 117, 156

blood cell count 36

body weight effects 21, 76

breast milk. $147,151,154$

cancer $4,5,13,38,85,99,110,111,130$

carcinogen 170

carcinogenic $14,22,170$ carcinogenicity..... cardiovascular . Cardiovascular Effects ..13 chromosomal aberrations..... 35,40

clearance 87

death. deoxyribonucleic acid (see DNA)

$91,92,115$ dermal effects. $12,17,18,19,24,35,40,84,86,100,163$ developmental effects ... DNA (see deoxyribonucleic acid). elimination rate endocrine. endocrine effects erythema.

fetus. $17,39,75,86,115$

follicle stimulating hormone (see FSH)

…..................... 75

FSH (see follicle stimulating hormone)

75, 80, 96, 98 gastrointestinal effects 11, 17, 35, 40, 102 general population $9,148,151,170$ genotoxic. $22,85,87$ genotoxicity groundwater $5,11,17,22,23,38,39,40,78,86,170$ $20,24,75,86,103$ $12,18,20,37,82,84,85,87,96,100,107,108,110,111,113,115,116$ .. 96, 101 $75,97,98$ half-life hematological effects hepatic effects ... 13

hydrolysis immune system $.3,85,130,134,139,143,159,161$
$\ldots . . . .11,35,91,92,100,105,140$ immunological immunological effects. $36,73,86$

$\mathrm{K}_{\mathrm{ow}}$ 36, 73

$\mathrm{LD}_{50}$ .. 124 114

lymphoreticular 22, 37, 76

milk 122, 123, 124 .39, 40 37 .74, 144, 154 


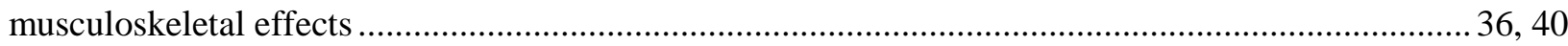

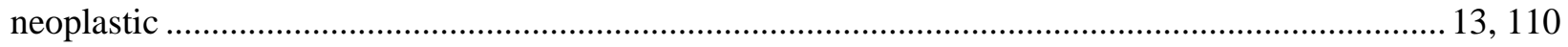

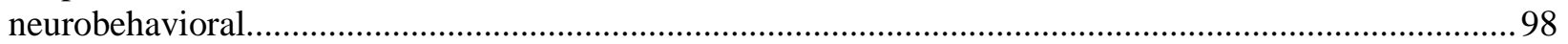

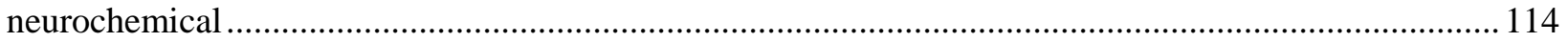

neurological effects ….................................................................... 37, 76, 77, 87, 102, 108, 114

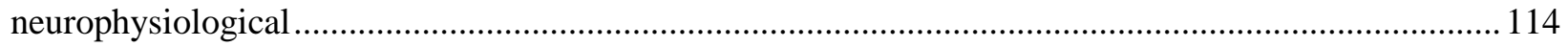

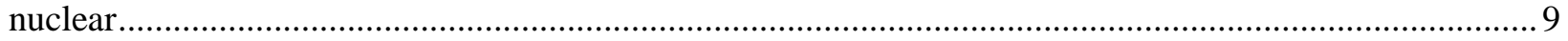

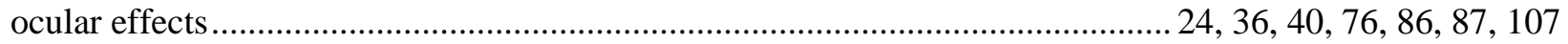

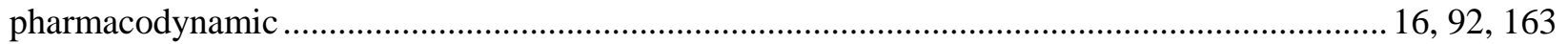

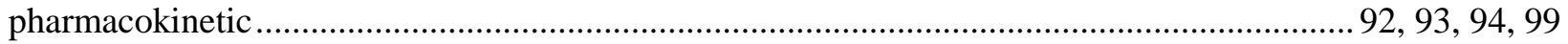

renal effects ................................................................................................. 24, 36, 74, 86, 96

reproductive effects................................................. 12, 37, 77, 79, 80, 81, 82, 84, 87, 96, 97, 113, 117

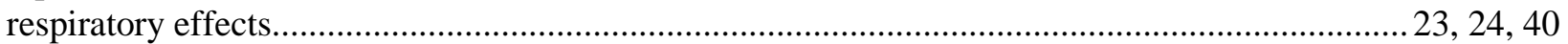

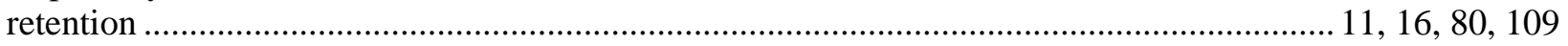

systemic effects...............................................................................12, 14, 21, 24, 40, 86, 101

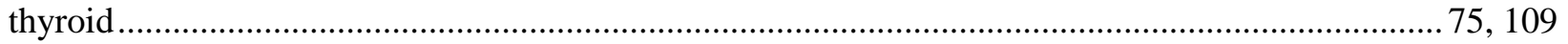

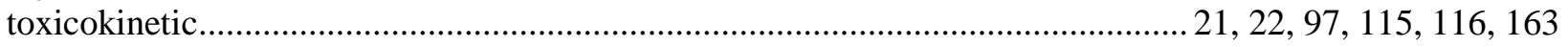

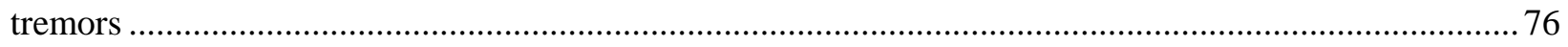

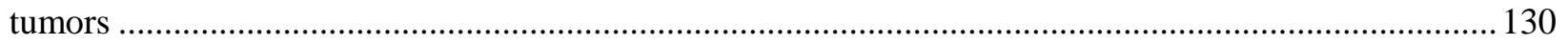


